\title{
Towards a unified view of inhomogeneous stellar winds in isolated supergiant stars and supergiant high mass X-ray binaries
}

\author{
Silvia Martínez-Núñez · Peter Kretschmar · Enrico \\ Bozzo · Lidia M. Oskinova - Joachim Puls · Lara \\ Sidoli · Jon Olof Sundqvist · Pere Blay · Maurizio \\ Falanga · Felix Fürst · Angel Gímenez-García . \\ Ingo Kreykenbohm · Matthias Kühnel · Andreas \\ Sander · José Miguel Torrejón · Jörn Wilms
}

Published at Journal of Space Science Reviews, Springer online 07 March 2017

\author{
S. Martínez-Núñez \\ Instituto de Física de Cantabria (CSIC-Universidad de Cantabria), E-39005, Santander, Spain E-mail: \\ silvia.martinez.nunez@gmail.com \\ P. Kretschmar \\ European Space Astronomy Centre (ESA/ESAC), Science Operations Department P.O. Box 78, E-28691, \\ Villanueva de la Cañada, Madrid, Spain E-mail: Peter.Kretschmar@esa.int \\ E. Bozzo \\ ISDC, University of Geneva, Chemin dEcogia 16, Versoix, 1290, Switzerland E-mail: En- \\ rico.Bozzo@unige.ch
}

L.M. Oskinova

Institut für Physik und Astronomie, Universität Potsdam, Karl-Liebknecht-Str. 24/25, D-14476 Potsdam, Germany

J. Puls

Universitätssternwarte der Ludwig-Maximilians-Universität München, Scheinerstrasse 1, 81679, München, Germany

L. Sidoli

INAF, Istituto di Astrofisica Spaziale e Fisica Cosmica - Milano, via E. Bassini 15, I-20133 Milano, Italy

J.O. Sundqvist

Centro de Astrobiología, CSIC-INTA, Ctra. Torrejón a Ajalvir km.4, 28850 Madrid, Spain \& Instituut voor Sterrenkunde, KU Leuven, Celestijnenlaan 200D, 3001 Leuven, Belgium

P. Blay

Nordic Optical Telescope - IAC, P.O.Box 474, E-38700, Santa Cruz de La Palma Santa Cruz de Tenerife, Spain

M. Falanga

International Space Science Institute (ISSI), Hallerstrasse 6, CH-3012 Bern, Switzerland \& International Space Science Institute in Beijing, No. 1 Nan Er Tiao, Zhong Guan Cun, Beijing 100190, China

F. Fürst

Cahill Center for Astronomy and Astrophysics, California Institute of Technology, Pasadena, CA 91125, USA

A Gímenez-García

Instituto Universitario de Física Aplicada a las Ciencias y las Tecnologías, University of Alicante, P.O. Box 99, E03080 Alicante, Spain

I. Kreykenbohm 
Abstract Massive stars, at least $\sim 10$ times more massive than the Sun, have two key properties that make them the main drivers of evolution of star clusters, galaxies, and the Universe as a whole. On the one hand, the outer layers of massive stars are so hot that they produce most of the ionizing ultraviolet radiation of galaxies; in fact, the first massive stars helped to re-ionize the Universe after its Dark Ages. Another important property of massive stars are the strong stellar winds and outflows they produce. This mass loss, and finally the explosion of a massive star as a supernova or a gamma-ray burst, provide a significant input of mechanical and radiative energy into the interstellar space. These two properties together make massive stars one of the most important cosmic engines: they trigger the star formation and enrich the interstellar medium with heavy elements, that ultimately leads to formation of Earth-like rocky planets and the development of complex life. The study of massive star winds is thus a truly multidisciplinary field and has a wide impact on different areas of astronomy.

In recent years observational and theoretical evidences have been growing that these winds are not smooth and homogeneous as previously assumed, but rather populated by dense "clumps". The presence of these structures dramatically affects the mass loss rates derived from the study of stellar winds. Clump properties in isolated stars are nowadays inferred mostly through indirect methods (i.e., spectroscopic observations of line profiles in various wavelength regimes, and their analysis based on tailored, inhomogeneous wind models). The limited characterization of the clump physical properties (mass, size) obtained so far have led to large uncertainties in the mass loss rates from massive stars. Such uncertainties limit our understanding of the role of massive star winds in galactic and cosmic evolution.

Supergiant high mass X-ray binaries (SgXBs) are among the brightest X-ray sources in the sky. A large number of them consist of a neutron star accreting from the wind of a massive companion and producing a powerful X-ray source. The characteristics of the stellar wind together with the complex interactions between the compact object and the donor star determine the observed X-ray output from all these systems. Consequently, the use of $\mathrm{SgXBs}$ for studies of massive stars is only possible when the physics of the stellar winds, the compact objects, and accretion mechanisms are combined together and confronted with observations.

This detailed review summarises the current knowledge on the theory and observations of winds from massive stars, as well as on observations and accretion processes in wind-fed high mass X-ray binaries. The aim is to combine in the near future all available theoretical

Dr. Karl Remeis-Observatory \& ECAP, Universität Erlangen-Nürnberg, Sternwartstr. 7, D-96049 Bamberg, Germany

M. Kühnel

Dr. Karl Remeis-Observatory \& ECAP, Universität Erlangen-Nürnberg, Sternwartstr. 7, D-96049 Bamberg, Germany

A. Sander

Institut für Physik und Astronomie, Universität Potsdam, Karl-Liebknecht-Str. 24/25, D-14476 Potsdam, Germany

J. M. Torrejón

Instituto Universitario de Física Aplicada a las Ciencias y las Tecnologías, University of Alicante, P.O. Box 99, E03080 Alicante, Spain

J. Wilms

Dr. Karl Remeis-Observatory \& ECAP, Universität Erlangen-Nürnberg, Sternwartstr. 7, D-96049 Bamberg, Germany 
diagnostics and observational measurements to achieve a unified picture of massive star winds in isolated objects and in binary systems.

Keywords Massive stars $\cdot$ stellar outflows $\cdot$ X-ray binary $\cdot$ wind-fed systems $\cdot$ accretion processes $\cdot \mathrm{SgXBs} \cdot \mathrm{SFXTs}$

\section{Introduction}

Massive stars $\left(M_{\text {initial }} \gtrsim 10 M_{\odot}\right)$ play an important role in the evolution of star clusters and galaxies. Massive stars generate ionizing ultraviolet radiation, and heat the dust. The winds of massive stars, and their final explosions as supernovae or gamma-ray bursts provide a significant input of energy and chemically enriched matter into the interstellar medium (Kudritzki, 2002). Massive stars are among the most important drivers of cosmic evolution, they regulate star formation and, together with low-mass stars, enrich the interstellar medium with heavy elements. Among the bright X-ray sources in the sky a significant number consists of a compact object accreting from the wind of such massive stars. These winds are fast (with typical terminal velocities up to $2500 \mathrm{~km} \mathrm{~s}^{-1}$ ), dense (with mass-loss rates up to $\dot{M} \gtrsim 10^{-5}-10^{-7} M_{\odot} \mathrm{yr}^{-1}$ ), and driven by line scattering of the star's intense continuum radiation field. Examples of a system comprising a massive star and a compact object are Cyg X-1/HDE 226868, the first detected stellar-mass black hole, and Vela X-1, the prototype of wind accreting neutron star X-ray binaries. Both in isolated massive stars and in binary systems with accreting compact objects, the basic picture of the wind formation and wind accretion process has been established for decades. However, new findings concerning inhomogeneities in the massive star winds and the unexpectedly pronounced X-ray variability in some wind-fed binaries questioned our previous understanding of these systems.

The first quantitative description of line-driven stellar winds was provided in the seminal paper by Castor et al. (1975), which assumed a stationary, homogeneous, and spherically symmetric outflow. Later works (e.g., Owocki et al., 1988; Feldmeier et al., 1997b a; Dessart and Owocki, 2005) showed that the line-driven hot star winds are in fact unstable 1 to velocity perturbations (the so-called "line-driven instability", hereafter LDI), leading to high-speed rarefactions that steepen into strong shocks, whereby most material is compressed into spatially narrow 'clumps' (or shells in 1-D simulations) separated by large regions of much lower densities. The presence of clumps in the winds of massive stars is supported by numerous observational evidences in many different wavebands (see Hamann et al., 2008; Sundqvist et al., 2011, for comprehensive overviews). In numerical simulations, the LDI is observed to generate strong wind shocks, which provide a possible explanation (Feldmeier et al., 1997b. a) for the soft X-ray emission observed from "normal" (putatively single, non-magnetic) OB-stars, as well as for their lack of significant time-variability (see Nazé et al., 2013, for a recent review). Clumps affect several stellar wind diagnostics in a non-trivial way, and discussions are on-going to infer the physical properties of these structures from the results of the most recent observational campaigns.

Additional independent observational evidence of clumped stellar winds comes from supergiant high mass X-ray binaries ( $\mathrm{SgXBs}$ ), i.e. those systems in which a compact object (a black hole or a neutron star) orbits a supergiant O-B star. Sako et al. (2003) was the first to review spectroscopic results obtained by X-ray observatories for several wind-fed SgXBs. They concluded that the observed spectra and time variability of these objects could be best explained by assuming that accretion onto the compact object is taking place from

\footnotetext{
1 Already Lucy and Solomon (1970) pointed out that radiative line-driving is subject to a strong instability.
} 
a highly structured stellar wind where cool dense clumps are embedded in a rarefied photoionized gas. Similar studies were later carried out on a number of bright $\mathrm{SgXBs}$, including 4U 1700-37 (van der Meer et al., 2005), Vela X-1 (Kreykenbohm et al., 2008; Fürst et al., 2010; Martínez-Núñez et al., 2014), Cyg X-1 (Miškovičová et al., 2011), and GX 301-2 (Fürst et al., 2011). Although the presence of structured clumped winds in $\mathrm{SgXBs}$ seems thus well established, there is a still considerable uncertainty in the physical properties of those clumps and the mechanisms by which the structured wind is able to feed the compact object. Particularly puzzling is the pronounced X-ray variability (a factor of $\sim 100-1000$ higher than in classical $\mathrm{SgXBs}$ ) of the supergiant fast X-ray transients (SFXTs) sources. This variability is unlikely to be only due to the presence of massive structures in the wind of the supergiant stars and requires $a d-h o c$ assumptions on the on-going accretion processes.

The layout of the review is as follows: Section 2 first introduces the basic physics of line-driven winds in detail, from the pioneering 'CAK model' to modern simulations including small and large scale structures in the wind. The section continues with the theory of accretion of these winds onto compact objects and especially neutron stars, treating also different accretion regimes and the inhibition of accretion. Section 3 discusses the determination of stellar and wind parameters by quantitative spectroscopy in the optical and UV regime, including the effects of wind clumping on the mass loss diagnostics. Measurements and diagnostics in the X-ray regime are discussed in Section 4 together with caveats when applying these. This section also summarises the current knowledge on both the "classical" SgXBs and the SFXTs. Finally, Section 5 summarises the main currently open questions on stellar winds and wind properties of massive stars.

\section{Basic physics}

We summarise in the next two sections the basic physics of the line-driven winds in massive stars and accretion processes in wind-fed binaries.

\subsection{Basics of line-driven winds}

A decisive property of hot, massive stars is their stellar wind, with typical mass-loss rates (for solar metallicity), $\dot{M} \approx 10^{-7} \ldots 10^{-5} M_{\odot} / \mathrm{yr}$, and terminal velocities, $v_{\infty}$, ranging from $200 \ldots 3,500 \mathrm{~km} \mathrm{~s}^{-1}$. The origin of these winds is attributed to radiative line-driving, i.e., stellar continuum photons are scattered in a multitude of spectral lines and transfer their momentum to the wind. Since this process requires a large number of photons (i.e., a high luminosity), such winds occur in the hottest stars, like O-type stars of all luminosity classes, but also in cooler BA-supergiants, because of their larger radii. Efficient line-driving further requires a large number of spectral lines close to the flux-maximum and a high interaction probability (i.e., a significant optical depth). Since most spectral lines originate from various metals, a strong dependence of $\dot{M}$ on metallicity is thus to be expected, and such line-driven winds should only play a minor role (if at all) in the early Universe 2 The theory of line-driven winds has been pioneered by Lucy and Solomon (1970) and particularly by Castor et al. (1975, henceforth 'CAK'), with essential improvements regarding a quantitative description and application provided by Friend and Abbott (1986) and Pauldrach et al.

\footnotetext{
2 Contrasted to the almost metallicity-independent, porosity-moderated continuum-driven winds hypothesized by Owocki et al. (2004).
} 
(1986). Line-driven winds have been reviewed by Kudritzki and Puls (2000) and more recently by Puls et al. (2008). In the following, we will briefly consider some relevant aspects, mostly in terms of the 'standard model' and the theory developed by CAK.

\subsubsection{The CAK model and beyond}

From studying the temporal variability of typical wind-features (UV P-Cygni profiles, $\mathrm{H}_{\alpha}$, He II4686, see Sect. 3) and from analysing these lines, it turned out that the global quantities describing the outflow $\left(\dot{M}, v_{\infty}\right)$ typically show only little variations. This and other evidence motivates the definition of a stationary, spherically symmetric, and homogeneous standard model. Effects from rotation and magnetic fields are briefly outlined at the end of this section, and deviations from a homogeneous structure are discussed in Sects. 2.1.2 and 2.1 .3

In such a standard model, the mass-loss rate $\dot{M}=4 \pi r^{2} \rho(r) v(r)$ remains constant over the wind, and the equation of motion is governed by pressure terms and external forces, in our case the inward gravitational pull and an outward directed radiative acceleration. For simplicity, the Thomson-acceleration due to electron scattering will be included as a correction to gravity 3 in terms of the conventional Eddington-Gamma, $\Gamma_{\text {Edd }}=g_{\text {Thomson }} / g_{\text {grav }} \propto$ $L / M$, and the remaining continuum acceleration can be neglected in most hot star winds.

Thus, the 'only' difficulty regards calculating the radiative line force. Basically, this force can be derived from the momentum transfer occurring during the absorption and (re) emission of (mostly) stellar photons, where on average the emission process cancels out because of its fore-aft-symmetry. Since most photons are absorbed in metal lines, the momentum needs to be redistributed to the bulk plasma ( $\mathrm{H}$ and $\mathrm{He}$ ), by means of Coulomb collisions (Springmann and Pauldrach, 1992) 4 .

In certain frequency intervals, the line-density can be so high that photons on their way out of the wind are not only scattered in one line before they escape, but also in a second one, a third one, etc., until they ultimately find their way out. This process is called multi-line-scattering, and leads to a certain complexity in analytical calculations of the line force 5 For simplicity, we assume instead that each line can be treated separately, i.e., that stellar photons can interact with only one line and then leave the wind, irrespective of linedensity. As shown by Puls (1987), this is not too bad an approximation for OBA-stars 6 In this case, the total line force can be calculated by summing up the individual contributions from all participating lines, expressed in terms of illuminating intensity and line opacity. In rapidly expanding atmospheres, this expression can be simplified by means of the so-called Sobolev approximation (Sobolev, 1960). If at first we only consider radially streaming photons (relaxed later on), the radiative line-acceleration for line $i$ at rest transition-frequency $v_{0, i}$ results in

$$
g_{\mathrm{rad}, i}=\frac{L_{v_{i}} v_{0, i}}{c^{2}} \frac{\mathrm{d} v / \mathrm{d} r}{4 \pi r^{2} \rho}\left[1-\exp \left(-\frac{k_{\mathrm{L}, i} s_{\mathrm{e}} \rho v_{\mathrm{th}}}{\mathrm{d} v / \mathrm{d} r}\right)\right]=\frac{L_{v_{i}} v_{0, i}}{c^{2}} \frac{\mathrm{d} v / \mathrm{d} r}{4 \pi r^{2} \rho}\left[1-\mathrm{e}^{-\tau_{\mathrm{Sob}}}\right]
$$

\footnotetext{
${ }^{3}$ Both accelerations depend on $r^{-2}$, at least in a homogeneous medium.

${ }^{4}$ A significant drift between metallic ions and the bulk plasma or even a complete decoupling of certain ions might become possible in winds of low metallicity and/or low density, e.g., Babel (1995); Krtička et al. (2003); Krtička (2006); Owocki and Puls (2002).

5 For details, see, e.g., Friend and Castor (1983); Puls (1987); Lucv and Abbott (1993); Gavlev et al. (1995).

${ }^{6}$ In the dense winds of Wolf-Rayet stars (see below), multi-line scattering needs to be accounted for.
} 
with $L_{v_{i}}$ the spectral luminosity, $s_{\mathrm{e}}$ the mass-absorption coefficient for Thomson scattering, and $v_{\text {th }}$ the thermal velocity for a representative ion. $k_{\mathrm{L}, i}$ is the so-called line-strength, corresponding to the ratio between frequency-integrated line-opacity $\bar{\chi}_{i}$ and Thomson-scattering opacity $s_{\mathrm{e}}$ over a typical line-width $\Delta v_{\mathrm{Dop}, i}$,

$$
k_{\mathrm{L}, i}=\frac{\bar{\chi}_{i}}{s_{\mathrm{e}} \rho \Delta v_{\mathrm{Dop}, i}}
$$

For the dominating resonance lines from major ions, $k_{\mathrm{L}, i}$ is roughly constant over the wind. A line-strength of unity thus refers to a weak line of continuum electron-scattering strength, whereas strong lines can have $k_{\mathrm{L}, i} \approx 10^{6}$ or even more.

The most intriguing quantity appearing in Eq. 1 is the radial velocity gradient, which results from the Doppler-effect experienced by the absorbing matter in an expanding medium. As obvious from Eq. 1, the radiative acceleration from optically thin lines (with line optical depth in Sobolev approximation $\tau_{\mathrm{Sob}}<1$ ) is proportional to $k_{\mathrm{L}, i}$ and does not depend on velocity and density, whilst for optically thick lines $\left(\tau_{\mathrm{Sob}}>1\right) g_{\mathrm{rad}, i}$ becomes independent of line-strength (saturation), but now depends on $(\mathrm{d} v / \mathrm{d} r) / \rho$.

The basic trick of CAK was to write the total line acceleration, i.e., the sum over all contributing lines $i$, as an integral over a line-strength distribution

$$
g_{\mathrm{rad}}^{\mathrm{tot}}=\sum_{i} g_{\mathrm{rad}, i} \rightarrow \iint g_{\mathrm{rad}}\left(k_{\mathrm{L}}, v\right) \mathrm{d} N\left(k_{\mathrm{L}}, v\right)
$$

where this distribution depends on line-strength and frequency. From some preliminary empirical arguments which have been confirmed meanwhile (e.g., Puls et al. 2000 and Fig. 1), CAK assumed a power-law distribution w.r.t. $k_{\mathrm{L}}$ and a frequential distribution $\propto 1 / v$,

$$
\mathrm{d} N\left(k_{\mathrm{L}}, v\right)=-N_{0} k_{\mathrm{L}}^{\alpha-2} \mathrm{~d} k_{\mathrm{L}} \mathrm{d} v / v .
$$

In this case, integrals in Eq. 3 can be solved analytically, and one obtains

$$
g_{\text {rad }}^{\text {tot }}=\frac{L}{4 \pi r^{2} c^{2}} \frac{s_{\mathrm{e}} v_{\mathrm{th}} N_{0} \Gamma(\alpha)}{1-\alpha}\left(\frac{\mathrm{d} v / \mathrm{d} r}{s_{\mathrm{e}} v_{\mathrm{th}} \rho}\right)^{\alpha}:=\frac{s_{\mathrm{e}} L}{4 \pi r^{2} c} k_{\mathrm{CAK}} k_{1}^{\alpha}=g_{\text {grav }}(r) \Gamma_{\mathrm{Edd}} k_{\mathrm{CAK}} k_{1}^{\alpha},
$$

where $\Gamma(\alpha)$ is the Gamma-function,

$$
k_{\mathrm{CAK}}=\frac{v_{\mathrm{th}}}{c} \frac{N_{0} \Gamma(\alpha)}{1-\alpha}, \quad k_{1}=\frac{\mathrm{d} v / \mathrm{d} r}{s_{\mathrm{e}} \rho v_{\mathrm{th}}} .
$$

$k_{\text {CAK }}$ (on the order of 0.1 for O-stars and early B-stars) is one of the so-called forcemultiplier parameters, and $k_{1}$ the line-strength where the exponent in Eq. 1, the optical depth in Sobolev approximation, becomes unity $7 \alpha$ is the 2 nd force-multiplier parameter $(\approx 0.6 \ldots 0.7$ for O-star winds, see Fig. 1, left panel), either corresponding to the slope of the line-strength distribution function, Eq. 4 or alternatively interpreted as the ratio of lineacceleration from optically thick lines to the total line acceleration.

After accounting for non-radial photons and ionization effects (not discussed here), we can insert the total line acceleration into the time-independent equation of motion. The resulting non-linear differential equation can be solved either numerically (e.g., Pauldrach et al. 1986; Friend and Abbott 1986) or, applying certain simplifications, also analytically (e.g.,

\footnotetext{
$7 k_{1}$ corresponds to $t^{-1}$ in the notation of CAK.
} 

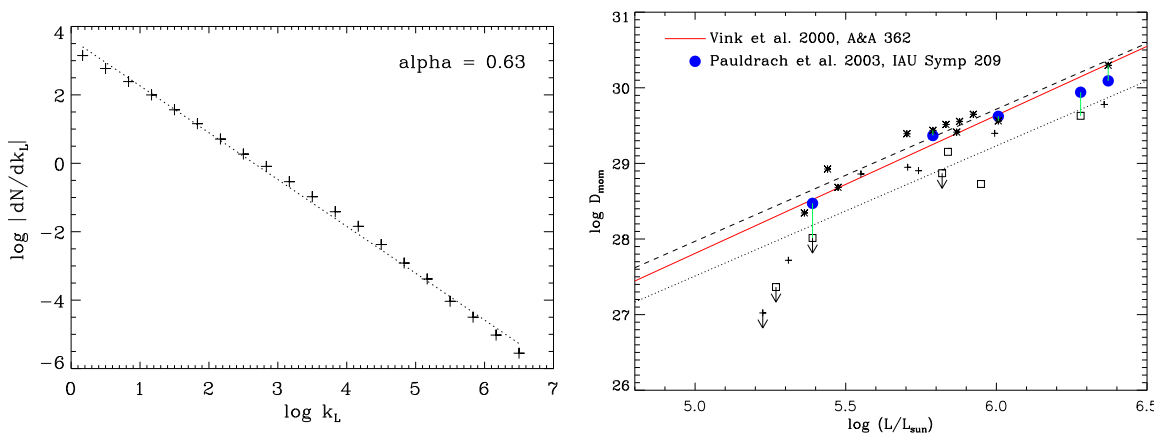

Fig. 1 Left: Frequency integrated line-strength distribution function for an O-type wind ( $T_{\text {eff }}=40 \mathrm{kK}$, solar abundance), and corresponding power-law fit (slope $=\alpha-2$ ). Right: Observed WLR for Galactic O-stars (asterisks, plus-signs and rectangles for luminosity classes I, III and V objects, respectively). Data from Puls et al. (1996). Dashed: linear regression to l.c. I objects; dotted: linear regression to luminosity class. III and V objects. Analytical considerations (see text) and theoretical models (from Vink et al. 2000, solid red) do not predict a dependence on luminosity class. The 'observed' difference is presumably due to the neglect of wind-inhomogeneities (clumping) in the mass-loss analysis (see also Repolust et al. (2004)). Note that the observed wind-momenta deviate towards low values below $\log L / L_{\odot}<5.2$ ('weak wind problem', cf. Marcolino et al. 2009 and references therein, and Huenemoerder et al. 2012 for a potential explanation). Blue dots indicate theoretical models from Pauldrach et al. (2003) calculated for five stars from the observed sample, resulting in similar wind-momenta as predicted by Vink et al. (2000).

Kudritzki et al. 1989; Owocki et al. 2004), and the mass-loss rate results as the eigenvalue of the problem. Overall, we obtain the following scaling relations:

$$
\begin{aligned}
\dot{M} & \propto\left(k_{\mathrm{CAK}} \frac{L}{L_{\odot}}\right)^{1 / \alpha^{\prime}}\left(\frac{M}{M_{\odot}}\left(1-\Gamma_{\mathrm{Edd}}\right)\right)^{1-1 / \alpha^{\prime}} \propto k_{\mathrm{CAK}}^{1 / \alpha^{\prime}} \frac{L}{L_{\odot}}\left(\frac{\Gamma_{\mathrm{Edd}}}{1-\Gamma_{\mathrm{Edd}}}\right)^{1 / \alpha^{\prime}-1} \\
v(r) & =v_{\infty}\left(1-\frac{R_{*}}{r}\right)^{\beta} \\
v_{\infty} & \approx \frac{2.25 \alpha}{1-\alpha}\left(\frac{2 G M\left(1-\Gamma_{\mathrm{Edd}}\right)}{R_{*}}\right)^{\frac{1}{2}}=\frac{2.25 \alpha}{1-\alpha} v_{\mathrm{esc}}
\end{aligned}
$$

Here, $\alpha^{\prime}=\alpha-\delta$, where $\delta(\approx 0.1$ for O-stars $)$ is Abbott's $(1982)$ ionization parameter, and $v_{\text {esc }}$ is the photospheric escape velocity, corrected for the radiative acceleration by Thomson scattering. The exponent $\beta$ is on the order of 0.8 for O-dwarfs, and on the order of $1.3 \ldots 2$ for BA-supergiants. To overcome some inherent problems with this initial CAK formulation (e.g., the artificial dependence on a fiducial thermal speed, see above), Gayley et al. (1995) used a somewhat different definition for the line-strength, as well as a line-statistics with an exponential cut-off, and reformulated the standard CAK approach. While the two formulations give identical results, the new one provides a somewhat modified expression for the mass-loss rate, which is frequently used nowadays:

$$
\dot{M} \propto\left(\frac{L}{L_{\odot}}\right)^{1 / \alpha^{\prime}}\left(\frac{1}{\bar{Q}} \frac{M}{M_{\odot}}\left(1-\Gamma_{\text {Edd }}\right)\right)^{1-1 / \alpha^{\prime}} \propto \frac{L}{L_{\odot}}\left(\frac{\bar{Q} \Gamma_{\text {Edd }}}{1-\Gamma_{\text {Edd }}}\right)^{1 / \alpha^{\prime}-1}
$$

For $\delta=0$, the relation between $\bar{Q}(\approx 2000$ for O-stars $)$ and $k_{\mathrm{CAK}}$ is given by $\bar{Q}^{1 / \alpha-1}=$ $c / v_{\mathrm{th}}\left((1-\alpha) k_{\mathrm{CAK}}\right)^{1 / \alpha}$.

Finally, by using the scaling relations for $\dot{M}$ and $v_{\infty}$ (Eqs. 7), and approximating $\alpha^{\prime} \approx$ $2 / 3$, one obtains the so-called wind-momentum luminosity relation - WLR - Kudritzki et al., 
1995),

$$
\log D_{\text {mom }}=\log \left(\dot{M} v_{\infty}\left(\frac{R}{R_{\odot}}\right)^{\frac{1}{2}}\right) \approx \frac{1}{\alpha^{\prime}} \log \left(\frac{L}{L_{\odot}}\right)+\operatorname{offset}(Z, \text { spectral type }),
$$

which relates the modified wind-momentum rate $D_{\text {mom }}$ with the stellar luminosity alone. The dependence on $M$ and $\Gamma_{\text {Edd }}$ (difficult to 'measure') vanishes since the product of $(M(1-$ $\left.\left.\Gamma_{\text {Edd }}\right)\right)^{1-1 / \alpha^{\prime}}$ and $\left(v_{\text {esc }} R^{1 / 2}\right)$ becomes negligible as long as $\alpha^{\prime}$ is close to $2 / 3$. The offset in Eq. 8depends on metallicity and spectral type, mostly because the effective line number and thus $k_{\mathrm{CAK}}$ (or $\bar{Q}$ ) depend on these quantities, via different opacities and contributing ions.

Originally, it had been suggested to use a carefully calibrated WLR as an independent tool to measure extragalactic distances, from the spectroscopic analysis of extragalactic Asupergiants and their winds, and by solving for the stellar radius via Eq. 8 Meanwhile, however, the WLR is mostly used to test the validity of the line-driven wind theory itself (e.g., Fig.11 right panel).

Various theoretical models have been computed during recent decades, based on a more or less exact calculations of the line-force (i.e., discarding the statistical approach and accounting for non Local Thermodynamic Equilibrium (hereafter, non-LTE) effects). Most prominent are the models by Vink et al. (2000, 2001), relying on a Monte Carlo approach, the models by Pauldrach (1987) and Pauldrach et al. (1994, 2001) ('WM-Basic'), calculating the line-force in a Sobolev-approach, and the models by Krtička and Kubát (2000, 2001, 2004), which include a more-component description (metal ions plus $\mathrm{H} / \mathrm{He}$ ). All these models agree in their quantitative predictions (e.g., Fig. 1 right panel), in particular regarding the metallicity dependence of the mass-loss rate, $\dot{M} \propto Z^{0.6 \ldots 0.7} 8$

The most impressive observational confirmation of the theoretical concept of line-driven winds and their metallicity dependence has been provided by Mokiem et al. (2007), compiling observed stellar- and wind-parameters from Galactic, LMC and SMC O-stars, and analysing the corresponding WLRs. Accounting for wind-inhomogeneities (see Sect.2.1.2) in an approximate way, they derive $\dot{M} \propto Z^{0.72 \pm 0.15}$, in very good agreement with theoretical predictions.

The bi-stability jump One of the still unsolved problems regarding line-driven winds is the reality of the so-called bi-stability jump? which should affect the mass-loss rates of Bsupergiants (important in the context of SgXBs). As it turns out (Puls et al., 2000; Vink et al., 2001), the mass-loss rates of radiation driven winds are mostly determined by iron (group) lines. Below $T_{\text {eff }} \approx 23 \mathrm{kK}$, the ionization of Fe (in the lower wind) switches abruptly from Fe IV to Fe III, which has much more lines close to flux maximum. Consequently, the massloss rate is predicted to increase for such cooler stars by roughly a factor of five or more, whilst $v_{\infty}$ should decrease by a factor of two (Vink et al., 2001). Thus, the wind-momentum rates for B-supergiants (like in Vela X-1) are predicted to be larger than of O-stars of similar luminosity.

Though a gradual decrease in $v_{\infty}$ (more precisely, in the ratio $v_{\infty} / v_{\text {esc }}$ ) over the range $T_{\text {eff }}=23 \mathrm{kK}$ to $18 \mathrm{kK}$ has been confirmed in many studies (e.g., Groenewegen et al. 1989; Crowther et al. 2006; Markova and Puls 2008), this is not true for the predicted increase in $\dot{M}$. Detailed investigations of B-supergiants by Crowther et al. (2006) and Markova and Puls (2008) do not show such a behaviour, but rather indicate that their mass-loss rates are lower (or similar) to those from O-stars at the same luminosity. Since the theoretical predictions

\footnotetext{
8 The metallicity dependence of $v_{\infty}$ is rather weak, $v_{\infty} \propto Z^{0.06 \ldots 0.13}$ (Leitherer et al., 1992; Krtička, 2006).

9 introduced by Pauldrach and Puls (1990) to explain the bi-stable behaviour of wind models for P Cygni.
} 
are quite robust, whereas the formation of the prime mass-loss indicator, $\mathrm{H}_{\alpha}$, is quite complex in the B-supergiant range (Petrov et al., 2014), further investigations are required to solve this long-standing issue. One might note, however, that all present evolutionary codes for massive stars incorporate the theoretical mass-loss predictions, and that the predicted bi-stability jump has a large effect on the evolution and rotation of B-supergiants and beyond (Vink et al., 2010; Markova et al., 2014). If the jump in $\dot{M}$ were not present, significant changes in such evolutionary phases are to be expected.

Mass loss from Wolf-Rayet stars From early on, the mass-loss properties of Wolf-Rayet stars posed a major problem for theoretical explanations, since they are considerably larger compared to O-stars of similar luminosity. Though Lucy and Abbott (1993) showed that line-overlap effects, coupled with a significantly stratified ionization balance, can help a lot to increase the mass-loss, it were Gräfener and Hamann (2005, 2006, 2007) who showed that there are two ingredients that might produce the observed large mass-loss rates in parallel with high terminal velocities. First, a high Eddington- $\Gamma$ is necessary to provide a low effective gravity and to enable a deep lying sonic point at high temperatures. Then, a high mass-loss rate leading to an optically thick wind can be initiated either by the 'hot' $\mathrm{Fe}$ opacity bump (around $160 \mathrm{kK}$, for the case of WCs and WNEs) or the cooler one (around 40 to $70 \mathrm{kK}$, for the case of WNLs) 10 Alternative wind models have been constructed by Vink et al. (2011), who argue that for $\Gamma_{\text {Edd }}>0.7$ the winds (more precisely, the pseudocontinuum) become optically thick already at the sonic point, which should enable a high $\dot{M}$. Nevertheless, there are still a number of details to be worked out before these winds are completely understood.

Impact of (fast) rotation When stars rotate rapidly, their photospheres become oblate, the effective temperature decreases from pole towards equator ('gravity darkening'), and the wind is predicted to become prolate in most cases (because of the larger illuminating polar fluxes), with a fast and dense polar outflow, and a slow and thinner equatorial one (Cranmer and Owocki, 1995) 11 Whilst stellar oblateness and gravity darkening have been confirmed (at least the basic effects) by means of interferometry (Domiciano de Souza et al., 2003; Monnier et al., 2007), the predictions on the wind-structure of rapidly rotating stars have not been verified by observations so far (Puls et al. 2010 and references therein): first, only few stars in such phases are known (but they exist, e.g., the most extremely rotating massive star detected by Dufton et al. 2011 rotates very close to critical), and second, the tools to analyse the atmospheres and winds (multi-D models!) of such stars are rare, if they exist at all. Note that even for moderate rotation the wind is predicted to become asymmetric (though to a lesser extent), and the formation of important mass-loss diagnostics such as $\mathrm{H}_{\alpha}$ becomes affected (e.g., Petrenz and Puls 1996).

Impact of magnetic fields Recent spectropolarimetric surveys (mostly performed by the international Magnetism in Massive Stars, MiMeS, collaboration, e.g., Wade et al. 2012, and work done by S. Hubrig and collaborators, e.g., Hubrig et al. 2013) have revealed that roughly $10 \%$ of all massive stars have a large-scale, organized magnetic field in their outer stellar layers (the incidence of internal fields might be higher), on the order of a couple of hundred to several thousand Gauss. The origin of these fields is still unknown, though most

10 The importance of these opacity bumps was pointed out already by Nugis and Lamers (2002).

11 All these effects become significant if the rotational speed exceeds roughly $70 \%$ of the critical one. 
evidence points to quite stable fossil fields formed sometimes during early phases of stellar formation (Alecian et al., 2013). The interaction of these fields with the stellar wind has been theoretically investigated by ud-Doula, Owocki and co-workers in a series of publications (summarised in ud-Doula 2013), and two different scenarios have been identified, depending on rotational speed and field strength. For not too fast rotation (when the Alvén radius is smaller than the Keplerian co-rotation radius), a magnetically confined wind is predicted, in which the gravitational pull on the trapped wind plasma creates large regions of infalling material, whereas for fast rotation and strong confinement12 one obtains a rigidly rotating magnetosphere (Alvén radius larger than Keplerian radius ( these radii are defined in Sect.2.2.2), in which the centrifugal force prevents the trapped material from falling back to the stellar surface. Both scenarios are consistent with observational findings (Petit et al., 2013), and are nowadays called dynamical and centrifugal magnetospheres. These two populations can be differentiated by their distinct $\mathrm{H}_{\alpha}$ emission: slowly rotating O-type stars with narrow, strong emission consistent with a dynamical magnetosphere, and more rapidly rotating B-type stars with broader, often double-peaked, emission associated with a centrifugal magnetosphere. First attempts (Sundqvist et al., 2012a) to simulate the $\mathrm{H}_{\alpha}$ emission from the dynamical magnetospheres of prototypical O-stars (denoted by the spectral type qualifiers 'f?p') have been quite successful, thought these initial investigations certainly need to be repeated within a multi-D NLTE approach.

\subsubsection{Small scale structures}

Although the standard theory of line-driven winds outlined in the previous section assumes a stable, time-independent and homogeneous wind, it is since long known that the radiation line-force in fact is subjected to a very strong, intrinsic instability (Milne, 1926; Lucy and Solomon, 1970). Below we review the theoretical background for this fundamental instability, whereas the corresponding observational background regarding small-scale wind structure is given in Sect. 3.4

Linear perturbation theory Following Owocki and Rybicki (1984), let us assume a small velocity perturbation of the conventional sinusoidal form $\delta v=\delta v_{0} e^{i(k x-w t)}$, where the wave number $k$ is the inverse of the perturbation wavelength and the circular frequency $w$ may be complex (to account for potential exponential growth or damping of the initial perturbation). For a spherically symmetric wind, in a frame co-moving with the underlying mean flow, and neglecting gas pressure terms, this circular frequency is given by

$$
w=i \delta g_{\mathrm{rad}} / \delta v \text {, }
$$

where $\delta g_{\mathrm{rad}}=g_{\mathrm{rad}}-g_{\mathrm{rad}, 0}$ is the response of the unperturbed line force $g_{\mathrm{rad}, 0}$ to the velocity perturbation. For a line that is optically thick in the mean flow, Owocki and Rybicki (1984) showed the ratio $\delta g_{\text {rad }} / \delta v$ can be expressed by a "bridging law"

$$
\frac{\delta g_{\mathrm{rad}}}{\delta v} \approx \Omega i k \frac{L_{\mathrm{Sob}}}{1+i k L_{\mathrm{Sob}}},
$$

where $\Omega \approx v_{0} / L_{\mathrm{Sob}}$ is the growth rate of the perturbation, and $L_{\mathrm{Sob}}=v_{\mathrm{th}} /\left(d v_{0} / d r\right)$ the radial Sobolev length 13 of the unperturbed flow, moving with $v_{0}$ in the stellar frame. Thus,

\footnotetext{
12 For example, a large ratio between magnetic and wind energy.

13 the radial extent of that zone where photons can be absorbed by a specific line.
} 
for large-scale perturbations with wavelengths longer than this Sobolev length $\left(k L_{\mathrm{Sob}}<<1\right)$,

$$
w \approx i^{2} \Omega k L_{\mathrm{Sob}} \approx-k v_{0} .
$$

Inserting this into the perturbation $\delta v=\delta v_{0} e^{i(k r-w t)}$ gives rise to radiative-acoustic waves of zero growth and with phase speed $w / k \sim-v_{0}$, propagating backwards in the co-moving frame. The properties of such "Abbott waves" (Abbott, 1980) thus imply that a line-force computed within the Sobolev approximation (like for all models discussed in the previous section) is marginally stable.

However, for short wavelength perturbations near or below this Sobolev length $\left(k L_{\mathrm{Sob}}>\right.$ 1), we obtain instead

$$
w \approx i \Omega \approx i \frac{v_{0}}{L_{\mathrm{Sob}}},
$$

which is complex with $\mathfrak{I}(w)>0$, and so when inserted into the perturbation-expression above results in an exponential growth of the initial velocity perturbation. Since the growth rate $\Omega \sim v_{0} / L_{\text {Sob }} \sim v_{0} / v_{\text {th }}\left(d v_{0} / d r\right)$ is a factor $\sim v_{0} / v_{\text {th }}$ larger than the wind expansion rate $d v_{0} / d r$, this implies small-scale perturbations can be amplified by an enormous amount $v_{0} / v_{\text {th }} \sim 100$ e-folds within this linear theory for a pure absorption-line-driven flow!

This strong line-deshadowing instability (LDI 14 can be somewhat damped by asymmetries in the scattered, diffuse component of the line-force (Lucy,, 1984). Owocki and Rybicki (1985) (see also Owocki and Puls 1996) showed that accounting for the diffuse force in the perturbation theory outlined above still gives a very un-stable outer wind, but where the LDI growth rate can be strongly damped close to stellar surface, and even become zero at the photospheric boundary. Recently, Sundqvist and Owocki (2013) showed that including simple stellar limb-darkening breaks this cancellation of the LDI by the diffuse damping at the stellar surface, and so leads to a net instability growth rate and to an unstable wind also in near photospheric layers.

Non-linear numerical simulations The operation of this fundamental and remarkably strong LDI has been confirmed by time-dependent numerical hydrodynamical wind modelling using a non-Sobolev radiation line-force (Owocki et al., 1988; Feldmeier, 1995; Owocki and Puls, 1999; Dessart and Owocki, 2005; Sundqvist and Owocki, 2013). Such simulations show that the non-linear growth of the LDI leads to high-speed rarefactions that steepen into strong reverse shocks (resulting, e.g., in high-energy emission observable in soft X-ray band-passes around $\sim 1 \mathrm{keV}$, see also below), whereby the wind plasma becomes compressed into spatially narrow 'clumps' separated by large regions of rarefied gas. This characteristic structure is the theoretical basis for our current understanding and interpretation of wind clumping (see Sect. 3).

The left panel in Fig. 2 2 illustrates this typical structure by plotting density, velocity, and temperature snapshots of a spherically symmetric LDI simulation computed from an initial steady Sobolev-based model following Sundqvist and Owocki (2013), and the right panel in the figure shows a contour-map of the density time evolution for the same model. The line force in this simulation is calculated using the "Smooth Source function" method (SSF, Owocki and Puls, 1996), which allows one to follow the non-linear evolution of the strong, intrinsic instability, while simultaneously accounting for the stabilizing effect of the scattered, diffuse radiation field. The simulation here further includes the effects of stellar limb darkening and photospheric density perturbations (the latter in the form of simple sound waves). The model displays clumps with very short characteristic radial length scales, on

\footnotetext{
14 or alternatively simply line-driven instability, also LDI.
} 

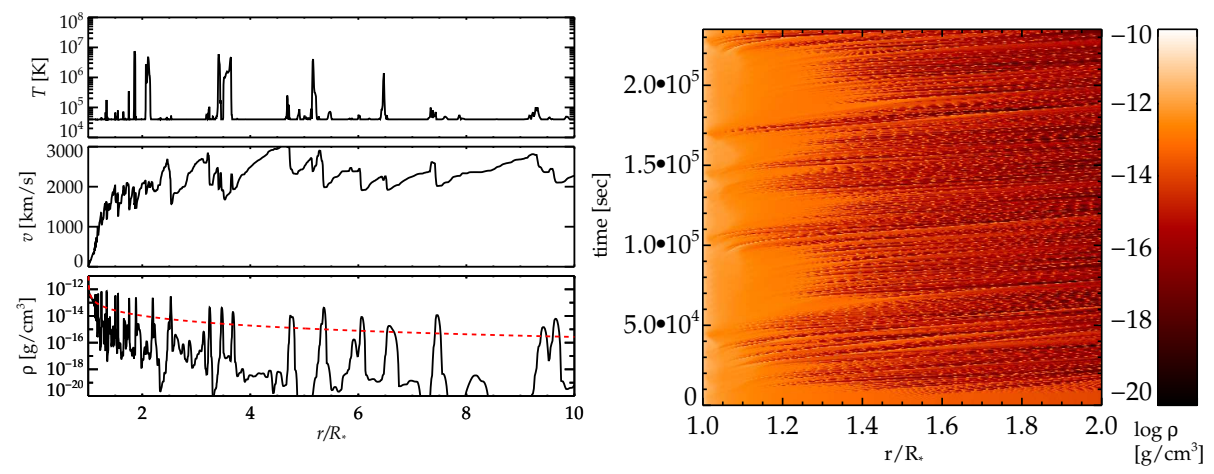

Fig. 2 Left: Temperature, velocity, and density snapshots of an LDI simulation (see text) that has reached a well-developed phase. The dashed red line in the bottom panel shows the density at $t=0 \mathrm{sec}$. Right: Contourmap of the inner-wind time-evolution of the density between $t=0$ and $t=23 \mathrm{ksec}$ in the same simulation (the characteristic wind flow time of this simulation is $t \approx R_{*} / v_{\infty} \approx 20 R_{\odot} / 2000 \mathrm{~km} / \mathrm{s} \approx 7 \mathrm{ksec}$ ).

the order of a Sobolev length $L_{\text {Sob }}$ or below (as expected from the linear theory above), and shows typical clump-densities an order of magnitude higher than the mean wind density. As can be seen from the figure, the rarefied regions in between the dense clumps are much more extended and with very low densities, on order a percent of the mean density or even lower. The inclusion of limb-darkening and photospheric perturbations further leads to strong, variable wind structure also close to the photosphere, at $r \approx 1.05-1.2 R_{\star}$ (in contrast to earlier simulations which typically had this onset only at $r \approx 1.5 R_{\star}$, e.g. Runacres and Owocki 2002). An X-ray study of the B0I star QV Nor, the massive donor of the 4U1538-52 binary system, established an upper limit of $\sim 1.25 R_{*}$ on the radial onset of clumping (Torreión et al., 2015). We note though, that although the basic properties and predictions of LDI-caused structure are robust, quantitative details regarding, e.g., the onset of structure and over-densities of clumps depend on various assumptions made in the computations of the source function and the line-force, on the level of photospheric perturbations, and on the dimensionality of the problem (see discussion in Sundqvist and Owocki 2013).

The presence of strong, embedded wind shocks in LDI simulations further provides a generally accepted explanation for the X-rays observed from single O-stars without very strong magnetic fields. The jump velocities are typically on order $\sim 300 \mathrm{~km} / \mathrm{s}$, giving characteristic temperatures $T \sim 10^{6} \mathrm{~K}$ and associated quite soft X-ray emission at $\sim 1 \mathrm{keV}$ and below. In 1-D simulations like those above, Feldmeier et al. (1997a) demonstrated that the velocity dispersion of individual shells ('clumps') results in collisional merging of such shells, which creates regions of relatively dense, hot gas and X-ray emission at levels quite comparable to those typically observed for single O-stars. More realistically though, thin-shell instabilities and associated effects can be expected to break up the spherical shell structure into a more complex, multi-dimensional structure. Because of the computational expense of calculating the line-force at each time step, however, radiation-hydrodynamical LDI simulations have generally been limited to 1-D. But first attempts to construct 2-D simulations have been carried out by Dessart and Owocki (2003, 2005). Such models typically show that the LDI first manifests itself as strong density compressions mimicking corresponding 1-D simulations. But as these initial shell structures are accelerated outwards, they become disrupted by velocity shearing and Rayleigh-Taylor and/or thin-shell instabilities, resulting in lateral structure all the way down to the grid-scale (see Dessart and Owocki 2003). However, it may 
well be that these initial 2-D models exaggerate somewhat the level of lateral disruption, as they do not yet properly treat the lateral component of the diffuse line-force. Presuming this could damp azimuthal velocity perturbations on scales below the lateral Sobolev length $L_{\phi}=v_{\mathrm{th}} r / v_{r}$ (Rybicki et al., 1990), lateral breakup may be prohibited below scales of order $\phi \approx L_{\phi} / r \approx v_{\text {th }} / v_{\mathrm{r}} \approx 0.5$ degrees. Further work is required to examine this important issue by an adequate incorporation of the lateral line-force into multi-D LDI simulations.

Characteristic clump mass With the discussed caveats in mind, we may nonetheless use the basic predictions of the ab-initio hydrodynamical wind simulations above to make a simple order of magnitude estimate of a characteristic clump mass: at a typical radius $r \approx 2 R_{*}$, the radial and lateral Sobolev lengths are on the same order, i.e. $d v_{r} / d r \approx v_{r} / r$, and so we estimate a clump volume by $V_{\mathrm{cl}} \sim L_{\text {Sob }}^{3} \sim\left(v_{\mathrm{th}} /\left(v_{r} / r\right)\right)^{3}$. We further assume the clump density is set by $\rho_{\mathrm{cl}} \approx\langle\rho\rangle f_{\mathrm{cl}}$ with a clumping factor (see further Sect. 3.2) $f_{\mathrm{cl}} \approx 10$ and mean density $\langle\rho\rangle=\dot{M} /\left(4 \pi r^{2} v_{r}\right)$ for an average stellar mass-loss rate $\dot{M}$. Thus a characteristic clumpmass is $M_{\mathrm{cl}} \approx \rho_{\mathrm{cl}} V_{\mathrm{cl}} \approx \dot{M} f_{\mathrm{cl}} v_{\mathrm{th}}^{3} r /\left(4 \pi v_{r}^{4}\right)$. Inserting typical numbers for a hot supergiant, $\dot{M} \approx 10^{-6} M_{\odot} / y r, v_{\mathrm{th}} \approx 10 \mathrm{~km} / \mathrm{s}, R_{\star} \approx 20 R_{\odot}$, and $v_{r} \approx 1000 \mathrm{~km} / \mathrm{s}$, then yields $M_{\mathrm{cl}} \approx 10^{18} \mathrm{~g}$. Although this indeed should be viewed only as a rough order of magnitude estimate, we note that since the LDI predicts small clumps, where the characteristic length scale is the Sobolev length, it is generally quite difficult to reconcile the small-scale structures predicted by basic line-driven wind theory with clump-masses that are much (i.e. orders of magnitude) higher than this estimate. This is further discussed in Sect. 4 in the context of empirical constraints obtained from X-ray observations of SgXBs.

\subsubsection{Large scale structures}

Besides the small scale structures discussed in the previous section, the winds from massive stars also harbour structures of larger scale, inferred predominantly from the so-called discrete absorption components (DACs).

Discrete absorption components are optical depth enhancements in the absorption troughs of unsaturated UV P Cygni profiles, observed in most O- and early B-star winds (Howarth and Prinja, 1989), and also in late B-supergiants 15 (Bates and Gilheany, 1990). Typically, these DACs accelerate towards the blue wing of the profile on time scales of a few days, becoming narrower as they approach an asymptotic velocity 16 Their acceleration and recurrence time scales are correlated with the star rotational period (Prinja, 1988; Henrichs et al., 1988; Kaper et al., 1999). Since the acceleration of most strong DACs is much slower than the mean wind acceleration (Prinja et al., 1992; Prinja, 1994), DACs might arise from a slowly evolving perturbation through which the wind material flows (higher density or lower velocity gradient/velocity plateau: Lamers et al. (1982), or both: Fullerton and Owocki (1992)). Although a spherically-symmetric disturbance most likely can be ruled out (Prinja, 1992; Howarth, 1992), the structure must cover a substantial fraction of the stellar disk in order to produce the observed strong absorption features.

Dynamical models - Co-rotating Interaction Regions Summarizing the above findings and arguments, DACs should originate from coherent structures of significant lateral extent,

\footnotetext{
15 Absorption components have been also found in at least one WN7 star (Prinia and Smith, 1992), and in the Balmer lines of the LBV P Cygni (Markova, 1986).

16 This asymptotic value is frequently used to estimate $v_{\infty}$ (e.g., Howarth and Prinja (1989) and Prinja et al. (1990)).
} 
with an increased optical line depth due to density and/or velocity field effects, and a clock coupled to stellar rotation. As a potential candidate mechanism which is compatible with all these constraints, Mullan (1984, 1986) suggested co-rotating interaction regions (CIRs), which are well-studied in the solar wind ${ }^{17}$ By means of 2-D time-dependent hydrodynamic simulations, Cranmer and Owocki (1996) investigated this scenario in detail for the case of a rotating $\mathrm{O}$-star wind. Their most promising model comprises photospheric disturbances due to a bright stellar spot in the equatorial plane 18 Because of this disturbance, the line acceleration becomes locally enhanced (more flux!). Consequently, the local mass loss increases, and thus the density already near the star. Further out in the wind, the effect of the disturbance weakens (since the opening angle of the disturbance decreases, and the radiation from undisturbed regions of the stellar surface begins to dominate), and the radiative force cannot accelerate the higher density material as strongly as the unperturbed wind. In summary, a stream of higher density and lower velocity, compared to the undisturbed mean flow, is generated. Where the faster mean flow collides supersonically with the slow material at its trailing edge, a CIR of enhanced density is formed, and a (non-linear) signal is sent back towards the star which forms a sharp, propagating discontinuity in the radial velocity gradient (see also Feldmeier and Shlosman 2000, 2002). In the stellar frame, this feature travels slowly outwards, and a velocity plateau-like structure is formed between the trailing discontinuity ('kink') and the CIR compression.

By calculating the corresponding line optical depths and time-dependent synthetic profiles, Cranmer and Owocki (1996) showed that these slowly moving kinks, together with their low velocity gradients $\left(\tau \propto(\mathrm{d} v / \mathrm{d} r)^{-1}\right.$, cf. Eq. 1), are the likely origin of the observed DACs rather than the CIRs themselves. Moreover, the apparent acceleration of the synthetic DACs was found to be slower than the acceleration of the mean wind (as required), and also their recurrence time scale is strictly correlated with $v_{\text {rot }}$ (because of the link of the CIRs and the rotating photosphere). On the other hand, no correlation between acceleration time scale and rotational speed appeared in the synthetic profiles, contrasted to the observational indications.

By means of an instructive kinematic investigation, Hamann et al. (2001) showed that the low drift rate of the DACs is not a consequence of the CIRs themselves, but a consequence of the difference between mean flow and the velocity field of the pattern in which the features form. An upstream propagating pattern (as the kink in the Cranmer-Owocki model) inevitably results in a wavelength drift with a slower apparent acceleration than displayed by features formed in the mean flow or within the CIR itself. Any of these drifts, however, were shown to be independent on the rotation rate, leaving the observed correlation (if actually present) still unexplained, at least if the CIRs are induced by disturbances locked to the stellar surface, as assumed by both Hamann et al. (2001) and Cranmer and Owocki (1996).

In a detailed study, Lobel and Blomme (2008) relaxed this assumption in order to allow for a quantitative analysis of the temporal evolution of DACs for the fast-rotating B0.5 Ib supergiant HD 64760, one of the targets of the IUE MEGA Campaign (see below). A large grid of 3-D models and dynamic spectra for different spot parameters (brightness, opening angle and velocity) has been computed, producing a best fit for a model with two spots of unequal brightness and size on opposite sides of the equator, with spot velocities being

\footnotetext{
17 Indeed, this suggestion was made already before all constraints were known.

18 to be regarded as a representative for other potential disturbances, e.g., localized magnetic fields or nonradial pulsations
} 
five times slower than $v_{\text {rot }} 19$ All basic conclusions of Cranmer and Owocki (1996) could be confirmed, particularly the importance of velocity plateaus in between kinks and CIRs. Moreover, models with non-locked spots displayed a correlation of DAC acceleration time scale and $v_{\text {rot }}$, since the internal clock is now set by the rotational speed of the spots, whilst a change in the stellar rotational speed modifies the underlying bulk flow (densities and velocities) via a different centrifugal acceleration such that the time-scales are no longer conserved when $v_{\text {rot }}$ is changed.

One may note that the above hydro-simulations have been performed by calculating the line-force in Sobolev approximation, because of computational feasibility. An improved approach regarding this aspect has been provided by Owocki (1999), who calculated CIR models based on a non-local line force calculated from a three-ray treatment. Also here, somewhat "rounded" kinks followed by a velocity plateau are present! Unfortunately, however, the line-driven instability (LDI, see Sect.2.1.2) effectively destroys all macro-structure, though this might be related to a still inadequate three-ray treatment and/or insufficient resolution, leading to too low a lateral damping. Certainly, future work is required to clarify these problems.

Rotational Modulations (RMs) The observed correlations of the DAC properties with rotational speed inspired the IUE MEGA Campaign (Massa et al., 1995), during which three prototypical massive stars (including HD 64760 from above) were monitored almost continuously over 16 days. Most importantly, a new type of variability was detected, namely periodic modulations in the UV wind lines, derived from the dynamic spectra of HD 64760 (Prinja et al., 1995). Similar features (though not as pronounced) have been detected in only two other stars, $\zeta$ Pup (Massa et al., 1995; ; Howarth et al., 1995) and $\xi$ Per (O7.5 III (n)((f)), Kaper et al. 1999). Note that these features occur in parallel with the conventional DACs.

The peculiar phase properties of the modulation features indicate the presence of absorbing material with the same phase at two different projected velocities. By means of a kinematical model and corresponding synthetic spectra, Owocki et al. (1995) and Fullerton et al. (1997) suggested that these features are formed in azimuthally extended, co-rotating and spirally-shaped wind structures linked to a surface density which is modulated by nonradial pulsations, and they are observational "evidence for co-rotating wind streams rooted in surface variations" (Owocki et al., 1995). Meanwhile, Lobel (2013) performed 3-D hydrosimulations to investigate the rotational modulation features. To fit the observations, the RMs required quite a regular 'spoke-like' radial density pattern that was simulated as a result from the action of pressure waves at the lower wind boundary. These structures are then maintained in the wind by an increased line acceleration in front of the (rotating) pressure wave.

Density contrast in CIRs and RMs With respect to one of our central topics, the interaction between (inhomogeneous) winds of supergiants and compact objects in SgXBs, the typical density contrast of CIRs and RMs is of prime importance. Though this value depends on the specific situation (size of spots, amplitude of sound-wave, rotation rate, etc.), the above hydro-simulations indicate a rather mild density contrast, namely over-densities of factors $\sim 1.3$ (Lobel and Blomme, 2008) to $\sim 3$ (Cranmer and Owocki, 1996) for the CIRs and a maximum over-density of a factor of 1.17 within $10 R_{*}$ for the RM region of HD 64760

\footnotetext{
19 Motivated by a suggestion from Kaufer et al. (2006) who detected non-radial pulsations in the photospheric lines of HD 64760. To explain the observed $\mathrm{H}_{\alpha}$ variability, they invoked the beat period between two of these periods (lower than the rotational one!) to be responsible for the CIRs in the wind.
} 
(Lobel, 2013). Together with the rather small filling factor, these inhomogeneities thus have only a small impact on density squared $\dot{M}$ diagnostics (see Sect. 3), on the order of 2 to 3\% in $\dot{M}$, to be compared with typical factors of $\sqrt{f_{\mathrm{cl}}} \sim \sqrt{10} \sim 3$ resulting from the small-scale structures discussed in the previous section. We note though, that since these large-scale structures must cover a substantial fraction of the stellar disc (see above), their individual masses can be very high despite their modest density-enhancements. Following the previous section, we may estimate $M_{\text {cir }} \sim R_{*}^{2} \delta R \rho_{\text {cir }} \sim R_{*}^{2} \delta R \dot{M} f_{\text {cir }} /\left(4 \pi r^{2} v_{r}\right) \sim 10^{21-22} g$, where we have assumed a geometrical CIR-height $\delta R \approx 0.1-1 R_{\star}$, and used the same numbers as before for a typical hot supergiant but now assuming a characteristic CIR over-density set by $f_{\text {cir }}=2$. These type of large-scale wind structures can thus have much higher individual masses than the small-scale structures normally associated with wind clumping (see also Sect.44.

\subsection{Wind accretion theory}

In $\mathrm{SgXBs}$, an O-B supergiant is gravitationally bound to a compact object and the latter can accrete a significant fraction of the stellar wind. In most of these systems, and for all the systems that are of interest for this review, the massive star does not fill its Roche lobe and thus an accretion disk is not expected to form around the compact object (see, e.g., Frank et al., 2002). In the remaining part of this review we thus restrict our discussion to purely windfed systems and consider only the case in which the compact object is a neutron star (by far the majority of $\mathrm{SgXBs}$ are proven to host such kind of compact objects, the only known exceptions being Cyg X-1, GRS 1915+105 and LMC X-1). We provide first a description of the simplest theoretical wind-accretion treatment, i.e. the so-called Bondi-Hoyle-Lyttleton (BHL) accretion theory, and then summarise the most recent developments. In this second part we identify different possible accretion regimes, depending on the neutron star properties (magnetic field, spin period), as well as on the physical parameters of the supergiant wind (mass outflow rate, velocity, density). We also provide a short section summarizing the basic physics of the interaction between the X-rays produced as a consequence of the accretion onto the compact object and the stellar wind. Finally, the obtained results using a simplified analytical treatment are compared to the achievements of recent numerical simulations.

\subsubsection{Bondi-Hoyle-Lyttleton accretion}

In the following, we briefly summarise elements of the BHL theory. For a detailed review, we refer the reader to Edgar (2004).

The original work by Hoyle and Lyttleton (1939) considered accretion by a point mass (star) passing through a nebulous cloud as a mechanism for terrestrial climate change. This work was later updated by Bondi and Hoyle (1944), who included in the analysis the presence of an accretion wake behind the point mass. All these results were first applied in the context of accreting X-ray binaries by Davidson and Ostriker (1973). Below, we follow their approach (see Oskinova et al., 2012; Bozzo et al., 2016, for details).

Let us consider a neutron star of mass $M_{\mathrm{NS}}$, travelling at a relative speed $v_{\text {rel }}$ through a gas density $\rho$. If $v_{\text {rel }}$ is supersonic, then the gas flows in as described by Bondi and Hoyle (1944). The mass accretion rate onto the compact object is given by:

$$
\dot{M}_{\mathrm{acc}}=\pi \zeta R_{\mathrm{acc}}^{2} v_{\mathrm{rel}} \rho(a, t)
$$


where $\rho(a, t)$ is the wind density as a function of the orbital separation $a$ and time $t$. $\zeta$ is a numerical factor introduced to correct for the radiation pressure and the finite cooling time of the gas. In moderately luminous X-ray sources $\zeta \lesssim 1$, and it is usually assumed $\zeta \equiv$ 1(Davidson and Ostriker, 1973). The Bondi-Hoyle radius, or "accretion radius"

$$
R_{\mathrm{acc}}=\frac{2 G M_{\mathrm{NS}}}{v_{\mathrm{rel}}^{2}}
$$

represents a measurement of the distance at which the neutron star is able to gravitationally focus and capture the surrounding material. At this radius, the supersonically inflowing gas from the supergiant companion passes through a bow-shock and begins to free-fall toward the compact object. The relative speed $v_{\text {rel }}$ can be expressed as

$$
v_{\mathrm{rel}}^{2}=v_{\mathrm{NS}}^{2}(a)+v_{\mathrm{w}}^{2}(a, t)
$$

where $v_{\mathrm{NS}}$ is the velocity of the neutron star at the orbital separation $a$ and $v_{\mathrm{w}}$ is the stellar wind velocity.

In non-stationary stellar winds, the wind density $\rho(a, t)$ and the wind velocity $v_{\mathrm{w}}(a, t)$ depend on time (see Section 2.1). Combining equations (13) and (14), the accretion rate onto the neutron star can be written as:

$$
\dot{M}_{\mathrm{acc}}=4 \pi \zeta \frac{\left(G M_{\mathrm{NS}}\right)^{2}}{v_{\mathrm{rel}}^{3}} \rho(a, t) .
$$

The accretion of matter onto the neutron star produces X-rays. Assuming a direct conversion of the kinetic energy gained by the infalling matter to radiation at the neutron star surface, the X-ray luminosity of the system can be expressed as:

$$
L_{\mathrm{X}}=\frac{G M_{\mathrm{NS}} \dot{M}_{\mathrm{acc}}}{R_{\mathrm{NS}}}
$$

where $R_{\mathrm{NS}}$ is the neutron star radius. In the following, we consider commonly used values for the neutron star parameters, i.e., $R_{6}$ for the neutron star radius in units of $10^{6} \mathrm{~cm}$ and $M_{1.4}$ the neutron star mass in units of $1.4 M_{\odot}$. A reasonable scale for the mass accretion rate obtained from Eq. 16 is $\dot{M}_{16}$, i.e., $\dot{M}_{\text {acc }}$ in units of $10^{16} \mathrm{~g} \mathrm{~s}^{-1}$. We can thus write:

$$
L_{\mathrm{X}} \simeq 2 \times 10^{36} \frac{M_{1.4} \dot{M}_{16}}{R_{6}} \mathrm{erg} \mathrm{s}^{-1}
$$

Using the fact that in the cases of interest the neutron star velocity $v_{\mathrm{NS}}$ is usually much lower than the wind velocity $v_{\mathrm{w}}$, Eq. 15 reduces to $v_{\text {rel }} \simeq v_{\mathrm{w}}$. We thus obtain:

$$
R_{\mathrm{acc}} \approx \frac{3.7 \times 10^{10}}{v_{8}^{2}} \mathrm{~cm}
$$

where $v_{8}$ is the wind velocity in units of $10^{8} \mathrm{~cm} \mathrm{~s}^{-1}$.

The predicted X-ray luminosity (Eq. 18) can be rewritten in terms of measurable properties of the massive star wind. If we approximate the wind from the supergiant star as smooth and spherically symmetric, then we have at the neutron star location (orbital separation $a$ ):

$$
\rho_{\mathrm{w}}(a)=\frac{\dot{M}_{\mathrm{w}}}{4 \pi a^{2} v_{\mathrm{w}}(a)} .
$$


Including this in Eq. 13 (with $\zeta \equiv 1$ ) we get:

$$
\frac{\dot{M}_{\mathrm{acc}}}{\dot{M}_{\mathrm{W}}}=\frac{1}{4} \frac{R_{\mathrm{acc}}^{2}}{a^{2}} .
$$

Using Eq. 21 and Eq. 19 in Eq. 18, we obtain:

$$
L_{\mathrm{X}}=2 \times 10^{35} \frac{\dot{M}_{-6}}{a_{10 d}^{2} v_{8}^{4}} \mathrm{erg} \mathrm{s}^{-1},
$$

where $\dot{M}_{-6}$ is the mass-loss rate of the primary star in units of $10^{-6} \mathrm{M}_{\odot} \mathrm{yr}^{-1}$, and $a_{10 \mathrm{~d}} \mathrm{~cm}$, is the orbital separation for a massive binary in which $M_{\mathrm{NS}}+M_{*}=30 M_{\odot}$ and the NS is on a circular orbit with a period of 10 days:

$$
a=4.2 \times 10^{12} P_{10 \mathrm{~d}}^{2 / 3} M_{30}^{1 / 3}=4.2 \times 10^{12} a_{10 \mathrm{~d}} .
$$

In the remaining part of this section, we omit from all equations the symbols $R_{6}$ and $M_{1.4}$, as the range spanned by these parameters are marginally affecting the results compared to the larger uncertainties on more relevant quantities (e.g., the wind velocity, mass loss rate, the neutron star spin period and its magnetic field).

The regime in which accretion at the rate defined by Eq.21 is allowed, is usually termed "direct accretion" and corresponds to the maximum achievable X-ray luminosity in a windfed neutron star $\mathrm{SgXB}$. It is interesting to note from Eq.21 that, even in the direct accretion regime, wind-fed binaries are relatively inefficient accreting systems because in all cases of interest $R_{\text {acc }} \ll a$ and thus only a tiny fraction of the total mass lost by the primary star can reach the surface of the compact object. Another interesting property of these systems can be deduced from Eq. 18 . As in wind-fed $\mathrm{SgXBs}$ no accretion disk is present to mediate the transport of matter, the relevant time scale for changes in the mass accretion rate onto the compact object is roughly comparable to the free-fall time at $R_{\text {acc }}$, i.e., a few to hundred seconds (Frank et al., 2002). This means that any change occurring in either the mass inflow rate from the supergiant star, or in the velocity/density of the wind around the neutron star, would produce a virtually immediate variation in the source X-ray luminosity. For this reason, the pronounced X-ray variability of $\mathrm{SgXBs}$ is usually ascribed to the presence of structures (clumps) in the winds of their supergiant companions. However there are a number of complications in the accretion process (see Sect.2.2.2) that makes the evaluation of the stellar wind properties from the variations of the X-ray luminosity in these systems challenging. We will discuss more these aspects in Sect.4.

\subsubsection{Different accretion regimes}

The simplified scenario depicted in Sect. 2.2.1 provides only a very rough estimate of the mass accretion rate that a neutron star can experience due to the capture of wind from its massive companion. In particular, the direct accretion regime cannot apply in all circumstances as the neutron star rotation and magnetic field can dramatically affect the plasma entry through the compact object magnetosphere and thus the resulting mass accretion rate. In this section we describe all the accretion regimes that a neutron star can experience depending on the different key system parameters (i.e. the mass-loss rate from the primary star, the stellar wind velocity, the neutron star spin period, the magnetic field and the orbital separation).

We also generalize the treatment by relaxing the assumption of Eq. 20 and assuming that the wind velocity and density at the location of the neutron star can be more generally a 
non-trivial function of time and of the separation between the primary star and the compact object, i.e. $\rho_{\mathrm{w}}=\rho_{\mathrm{w}}(a, t)$ and $v_{\mathrm{w}}=v_{\mathrm{w}}(a, t)$.

Neutron stars in massive binaries are known to be strongly magnetized, as their relatively young age (a few $10^{6} \mathrm{yr}$ ) would not allow for a significant decay of the dipolar magnetic field with which they were endowed at birth $\left(\mathrm{B} \gtrsim 10^{12} \mathrm{G}\right)$. The magnetosphere around the neutron star has a non-negligible effect on the development of the accretion flow and begins to completely dominate its dynamics once the material gets close to the so-called magnetospheric radius, $R_{\mathrm{M}}$. For wind accreting systems, $R_{\mathrm{M}}$ can be roughly estimated by equating the ram pressure of the material flowing toward the neutron star with the local magnetic pressure. Scaling to typical parameters, we obtain:

$$
R_{\mathrm{M}}=1.3 \times 10^{9} \rho_{-12}^{-1 / 6} v_{8}^{-1 / 3} \mu_{30}^{1 / 3} \mathrm{~cm}
$$

where $\rho_{-12}=\rho_{\mathrm{w}} / 10^{-12} \mathrm{~g} \mathrm{~cm}^{-3}, \mu=B_{\mathrm{NS}} R_{\mathrm{NS}}^{3}$ is the magnetic moment of the NS and $\mu_{30}=\mu /\left(10^{30}\right) \mathrm{G} \mathrm{cm}^{3}$ (i.e., considering $B_{\mathrm{NS}}=10^{12} \mathrm{G}$ ). The direct accretion regime described in Sect.2.2.1 requires that $R_{\mathrm{M}}<R_{\text {acc }}$, in such a way that the accretion flow has enough time to settle in a free-fall spherical symmetric motion before encountering the neutron star magnetosphere. Combining Eq. 24 and 19, we can see that the condition $R_{\mathrm{M}}<R_{\text {acc }}$ is violated if

$$
\mu_{30} \gtrsim 2.3 \times 10^{4} \rho_{-12}^{1 / 2} v_{8}^{-5} \text {. }
$$

Assuming reasonable values for the supergiant wind velocity ( $\left.v_{8} \simeq 0.5-2\right)$, it turns out that the magnetospheric radius can extend beyond the accretion radius only for very strongly magnetized neutron stars with $B_{\mathrm{NS}} \gtrsim 10^{14} \mathrm{G}$. As discussed by Bozzo et al. (2008), such magnetic field would not be completely unreasonable if it is assumed that "magnetars" are hosted in SgXBs (see, e.g., Rea and Esposito, 2011, for a recent review). The regime in which $R_{\mathrm{M}} \gtrsim R_{\text {acc }}$ is called the "magnetic inhibition of accretion" regime, as the magnetosphere of the neutron star in this case inhibits the gravitational focusing of the wind material and decreases the effective accretion rate onto the compact object. It is noteworthy that a similar scenario was also envisaged by a number of authors to predict the X-ray luminosity of isolated magnetars accreting through the interstellar medium (Harding and Leventhal, 1992; Mori and Ruderman, 2003; Toropina et al., 2006). Before entering the details of the $\mathrm{X}$-ray luminosity released during the magnetic inhibition of accretion, it is necessary to introduce also the concept of the corotation radius.

It is known since the early 70 s that accretion onto a magnetized neutron star cannot occur unperturbed if the rotation of the compact object is slow enough to allow $R_{\mathrm{M}}$ to reside within the corotation radius:

$$
R_{\mathrm{co}}=3.7 \times 10^{9} P_{s 2}^{2 / 3} \mathrm{~cm}
$$

(here $P_{s 2}$ is the neutron star spin period in units of $100 \mathrm{~s}$ ). $R_{\text {co }}$ represents the distance from the neutron star at which material attached to the magnetic field lines of the compact object (corotating with it) reaches a velocity comparable with the local Keplerian velocity. The condition $R_{\mathrm{M}}<R_{\mathrm{co}}$ thus ensures that the centrifugal force at $R_{\mathrm{M}}$ is low enough for the incoming material to be accreted onto the neutron star rather than ejected by its fast rotation. When $R_{\mathrm{M}}>R_{\mathrm{co}}$, the neutron star centrifugal gate closes and the "propeller" regime sets-in, halting the incoming flow and centrifugally inhibiting accretion (Illarionov and Sunyaev, 1975). By combining Eq. 26] and 24, we can see that this case occurs if

$$
P_{\mathrm{s} 2} \lesssim 0.2 v_{8}^{-1 / 2} \rho_{-12}^{-1 / 4} \mu_{30}^{1 / 2}
$$

We thus have to distinguish different possibilities. 
- The super-keplerian magnetic inhibition regime. This case applies when both conditions $R_{\mathrm{M}} \gtrsim R_{\text {acc }}$ and $R_{\mathrm{M}} \gtrsim R_{\mathrm{co}}$ are satisfied. As argued before, the magnetic barrier in this case halts the mass inflowing from the companion star and inhibits the gravitational focusing of this material within the accretion radius. Furthermore, the wind material that approaches the neutron star magnetosphere is pushed away from the compact object due to the centrifugal gate. In this regime, no accretion is possible and the lowest X-ray luminosity is achieved. Bozzo et al. (2008) argued that the main contribution to the X-ray luminosity in this case is due to shocks close to the magnetospheric radius and to the dissipation of the neutron star rotational energy. The two contributions can be estimated as

$$
L_{\text {shock }} \simeq \frac{\pi}{2} R_{\mathrm{M}}^{2} \rho_{\mathrm{w}} v_{\mathrm{w}}^{3}=2.7 \times 10^{30} \mu_{30}^{2 / 3} \rho_{-12}^{2 / 3} v_{8}^{7 / 3} \mathrm{erg} \mathrm{s}^{-1}
$$

and

$$
L_{\mathrm{sd} 1} \simeq \pi R_{\mathrm{M}}^{2} \rho_{\mathrm{w}} v_{\mathrm{w}}\left(R_{\mathrm{M}} \Omega\right)^{2} \simeq 3.5 \times 10^{30} \mu_{30}^{4 / 3} \rho_{-12}^{1 / 3} v_{8}^{-1 / 3} P_{\mathrm{s} 2}^{-2} \mathrm{erg} \mathrm{s}^{-1},
$$

respectively (here $\Omega=2 \pi / P_{\mathrm{S}}$ ).

- The sub-keplerian magnetic inhibition regime. This case applies when $R_{\mathrm{M}} \gtrsim R_{\mathrm{acc}}$ but $R_{\mathrm{M}}<R_{\mathrm{co}}$. At odds with the previous case, despite the lack of gravitational focusing within $R_{\text {acc }}$, the effective gravity at the neutron star magnetosphere points toward the compact object. The inflowing material is thus not pushed away by the rotating star but tends to be accreted at a rate that is regulated by the efficiency of magnetohydrodynamics instabilities at $R_{\mathrm{M}}$. As the stellar wind material is passing by the neutron star with high velocities $\left(v_{8} \simeq 1\right)$, the most relevant instability allowing matter to penetrate the compact object magnetosphere is the Kelvin-Helmholtz instability (hereafter KHI; Harding and Leventhal, 1992). The mass inflow rate through the magnetosphere resulting from the $\mathrm{KHI}$ is:

$$
\dot{M}_{\mathrm{KHI}}=2 \pi R_{\mathrm{M}}^{2} \rho_{\mathrm{ps}} v_{\mathrm{conv}}=2 \pi R_{\mathrm{M}}^{2} \rho_{\mathrm{ps}} v_{\mathrm{sh}} \eta_{\mathrm{KHI}}\left(\rho_{i} / \rho_{e}\right)^{1 / 2}\left(1+\rho_{i} / \rho_{e}\right)^{-1}
$$

where $v_{\text {conv }}$ is the typical velocity at which matter crosses the magnetospheric boundary, $\rho_{\mathrm{ps}}$ is the density of the wind material being shocked and passing through the shock in front of the neutron star magnetosphere, $v_{\mathrm{sh}}$ is the shear velocity between the wind and the neutron star magnetosphere, $\eta_{\mathrm{KHI}} \simeq 0.1$ is an efficiency parameter, and $\rho_{i}\left(\rho_{e}\right)$ is the density immediately inside (outside) $R_{\mathrm{M}}$. Bozzo et al. (2008) argued that $v_{\mathrm{sh}}=\max \left(v_{\mathrm{ps}}, v_{\mathrm{rot}}\right)$, depending on the position along the neutron star magnetosphere. Here $v_{\mathrm{ps}}$ is the matter post-shock velocity dominating close to the stagnation point, while $v_{\text {rot }}=2 \pi R_{\mathrm{M}} P_{\mathrm{s}}^{-1}$ is the magnetosphere rotational velocity dominating the shear away from the stagnation point. The ratio $\rho_{i} / \rho_{e}$ can be estimated numerically from the mass conservation equation $R_{\mathrm{M}}^{2} \rho_{e} v_{\text {conv }} \simeq R_{\mathrm{M}} h_{t} \rho_{i} v_{\mathrm{ff}}\left(R_{\mathrm{M}}\right)$. Here we considered that matter crossing the $\mathrm{KH}$ unstable layer is rapidly brought at corotation with the neutron star and free-falls toward its surface before being accreted. The height of the KHI layer is assumed to be $h_{t} \simeq R_{\mathrm{M}}$. Bozzo et al. (2008) discussed that this is a reasonable assumption as long as $\rho_{i} \lesssim \rho_{e}$, even though a full detailed study of the KHI in these conditions is still lacking. Under these assumptions, the X-ray luminosity in the sub-keplerian magnetic inhibition regime can be estimated as $\max \left(L_{\mathrm{KH} 1}, L_{\mathrm{KH} 2}\right)$, where

$$
L_{\mathrm{KH} 1}=2.0 \times 10^{35} \eta_{\mathrm{KH}} \mu_{30}^{2 / 3} \rho_{-12}^{2 / 3} v_{8}^{1 / 3}\left(\rho_{\mathrm{i}} / \rho_{\mathrm{e}}\right)^{1 / 2}\left(1+\rho_{\mathrm{i}} / \rho_{\mathrm{e}}\right)^{-1} \mathrm{erg} \mathrm{s}^{-1},
$$

and

$$
L_{\mathrm{KH} 2}=6.5 \times 10^{35} \eta_{\mathrm{KH}} P_{\mathrm{s} 2}^{-1} v_{8}^{-1} \rho_{-12}^{1 / 2} \mu_{30}\left(\rho_{\mathrm{i}} / \rho_{\mathrm{e}}\right)^{1 / 2}\left(1+\rho_{\mathrm{i}} / \rho_{\mathrm{e}}\right)^{-1} \mathrm{erg} \mathrm{s}^{-1} .
$$

In this regime, we thus expect to observe relatively faint $\mathrm{X}$-ray sources. 
- The supersonic propeller regime. When $R_{\mathrm{M}}<R_{\mathrm{acc}}$, the wind material is gravitationally focused within the accretion radius and fills the region between $R_{\mathrm{acc}}$ and $R_{\mathrm{M}}$. The magnetic gate is open and the properties of this envelope surrounding the neutron star are determined by the physical processes regulating the interaction between matter and magnetic field at $R_{\mathrm{M}}$ (Davies and Pringle, 1981). If radiative losses are negligible within the envelope, then the latter is in hydrostatic equilibrium and stationary on dynamical timescales (this requires a sufficiently low mass-loss rate from the primary star, see Eq. B2 in Bozzo et al., 2008). In this case the pressure and density within the envelope can be written as:

$$
\begin{gathered}
p(R)=\rho_{\mathrm{ps}} v_{\mathrm{ps}}^{2}\left[1+(1 /(1+n)) 8 R_{\mathrm{a}} / R\right]^{n+1} \\
\rho(R)=\rho_{\mathrm{ps}}\left[1+(1 /(1+n)) 8 R_{\mathrm{a}} / R\right]^{n} .
\end{gathered}
$$

If the condition $R_{\mathrm{M}} \gtrsim R_{\mathrm{co}}$ applies, accretion is, however, still inhibited by the centrifugal barrier and the system enters the supersonic propeller regime. As the rotation of the magnetosphere is supersonic, dissipations at $R_{\mathrm{M}}$ induce turbulent motions that convect the dissipated neutron star rotational energy up through the outer boundary of the envelope, where this energy is dissipated. In this case, $n=1 / 2$ and the correct location of $R_{\mathrm{M}}$ should be computed by equating the neutron star magnetic pressure with the atmosphere pressure given above. We obtain:

$$
R_{\mathrm{M}}=3.2 \times 10^{8} v_{8}^{2 / 9} \mu_{30}^{4 / 9} \rho_{-12}^{-2 / 9} \mathrm{~cm}
$$

(where it was assumed that $R_{\mathrm{M}} \ll R_{\text {acc }}$ ). In the supersonic propeller regime, the material around the neutron star is pushed away from the rotating magnetosphere and accretion cannot occur. Dissipations at $R_{\mathrm{M}}$ are expected to provide the largest contribution to the X-ray luminosity:

$$
L_{\mathrm{X}}=2 \pi R_{\mathrm{M}}^{2} \rho\left(R_{\mathrm{M}}\right) c_{\mathrm{S}}^{3}\left(R_{\mathrm{M}}\right) \simeq 8.2 \times 10^{34} v_{8}^{-1} \rho_{-12} \mathrm{erg} \mathrm{s}^{-1}
$$

( $c_{\mathrm{s}}$ is the sound velocity).

- The subsonic propeller regime. When both conditions $R_{\mathrm{M}}<R_{\mathrm{acc}}$ and $R_{\mathrm{M}}<R_{\mathrm{co}}$ are satisfied, it is usually believed that the envelope around the neutron star still cannot accrete at the full regime indicated by Eq. 13 until material is cooled down below a certain critical temperature. If cooling is not efficient, the system enters the subsonic propeller regime. This is one of the most discussed regimes of wind accretion and different authors have made different assumptions regarding physical processes occurring in this case.

The subsonic propeller regime was initially investigated by Davies et al. (1979) and Davies and Pringle (1981), using theoretical findings proposed by Elsner and Lamb (1977) and Arons and Lea (1976). The latter authors argued that, in a wind-fed system, the material accumulating around the neutron star magnetosphere cannot penetrate it until its temperature is low enough for the magnetohydrodynamic instabilities to be efficient at $R_{\mathrm{M}}$. Both Elsner and Lamb (1977) and Arons and Lea (1976) suggested the RayleighTaylor instability (hereafter RTI) to be the most efficient instability in transporting material across the neutron star magnetic field lines. A criterion to trigger the RTI was derived from first principles (Bernstein et al., 1958), assuming a situation of hydromagnetic equilibrium in which the fluid velocity vanishes at any point. Under these conditions, the boundary between the plasma and a vacuum magnetic field is unstable for the RTI if the temperature of the plasma is (see also, Ikhsanov and Pustil'nik, 1996; Ikhsanov, 2005):

$$
T<T_{\text {crit }}\left(R_{\mathrm{M}}\right) \simeq 0.3 T_{\mathrm{ff}}\left(R_{\mathrm{M}}\right)=0.3 G M_{\mathrm{NS}} m_{\mathrm{p}} / k R_{\mathrm{M}}
$$


Here, $m_{\mathrm{p}}$ is the proton mass and $k$ is the Boltzmann constant. In a more detailed treatment, it can be seen that $T_{\text {crit }}$ has also a non-negligible dependence on the longitude of the neutron star magnetosphere, with the equatorial region being the least stable zone. If the condition of Eq. 37 is not satisfied, accretion is largely inhibited. In this situation, the neutron star spins at subsonic velocities within the surrounding shell and loses its rotational energy due to friction. As long as the dissipated rotational energy of the neutron star keeps the material in the spherical shell hot, the subsonic propeller regime can be sustained and the structure of the shell can be approximated as adiabatic. The magnetospheric radius can thus be estimated by using $n=3 / 2$ in Eq. 34 .

$$
R_{\mathrm{M}}=3.8 \times 10^{9} v_{8}^{6 / 7} \mu_{33}^{4 / 7} \rho_{-12}^{-2 / 7} \mathrm{~cm} .
$$

In the subsonic propeller regime the luminosity produced by the dissipation of the neutron star rotational energy can be written as:

$$
\begin{array}{r}
L_{\mathrm{sd} 3}=2 \pi R_{\mathrm{M}}^{5} \rho\left(R_{\mathrm{M}}\right) \Omega^{3}=6.2 \times 10^{35} P_{\mathrm{s} 2}^{-3} . \\
\cdot R_{\mathrm{M} 10}^{5} \rho_{-12}\left(1+16 R_{\mathrm{acc} 10} /\left(5 R_{\mathrm{M} 10}\right)\right)^{3 / 2} \mathrm{erg} \mathrm{s}^{-1},
\end{array}
$$

where $R_{\mathrm{M} 10}=R_{\mathrm{M}} / 10^{10} \mathrm{~cm}$ is given by Eq. 38 , and $R_{\mathrm{acc} 10}=R_{\mathrm{acc}} / 10^{10} \mathrm{~cm}$. A number of different prescriptions for the X-ray luminosity produced in the subsonic regime were proposed by different authors, and we refer the reader to the most relevant publications for further details (see, e.g., Ikhsanov, 2001b. c. a, and references therein). It is noteworthy that in all cases the assumed key characteristics of this regime are a strong spin-down of the neutron star and a moderate X-ray luminosity $\left(\lesssim 10^{33}-10^{34} \mathrm{erg} \mathrm{s}^{-1}\right.$ Ikhsanov, 2001a, 2007).

The approach to the subsonic propeller regime discussed above was criticised by Burnard et al. (1983). These authors argued that, when the neutron star rotational velocity is taken into account, the basic assumptions behind Eq. 37 are no longer valid and different conclusions can be reached. In particular, the presence of a shear velocity between the neutron star magnetosphere and the material in the shell would excite the KHI. Even in those situations in which the RTI is not efficiently working due to the high temperature of the plasma, material could still be pushed through the compact object magnetic field lines by the KHI and be finally accreted onto its surface. Burnard et al. (1983) presented all calculations needed to estimate the amount of material that can be accreted through the KHI depending on the NS magnetic field and spin period (as well as from the physical conditions of matter outside the NS magnetosphere). Following the simplified treatment of Bozzo et al. (2008), the mass luminosity in the subsonic propeller regime permitted by the KHI can be written as:

$$
\begin{aligned}
& L_{\mathrm{KH} 3} \simeq G M_{\mathrm{NS}} \dot{M}_{\mathrm{KH}} / R_{\mathrm{NS}}=1.5 \times 10^{39} \eta_{\mathrm{KH}} P_{\mathrm{s} 2}^{-1} R_{\mathrm{M} 10}^{3} \rho_{-12} . \\
& \cdot\left(1+16 R_{\mathrm{acc} 10} /\left(5 R_{\mathrm{M} 10}\right)\right)^{3 / 2}\left(\rho_{\mathrm{i}} / \rho_{\mathrm{e}}\right)^{1 / 2}\left(1+\rho_{\mathrm{i}} / \rho_{\mathrm{e}}\right)^{-1} \mathrm{erg} \mathrm{s}^{-1}
\end{aligned}
$$

It is thus found that, even in the subsonic propeller regime, a significant amount of material can still be accreted onto the NS at odds with what was considered before. The conditions under which the RTI becomes inefficient and the KHI begins to control matter penetration through the neutron star magnetosphere are still highly debated and difficult to be assessed. The problem is that these conditions depend strongly on the physical properties of matter and magnetic field at the magnetospheric boundary, which is an extremely complex region to be treated analytically. The conditions for the development of magnetohydrodynamic instabilities in the boundary are also affected by the X-ray 
photon field produced by the central accreting neutron star. In most of the cases of interest for this review, the photon flux changes significantly on rapid time scales (few to hundreds of seconds), thus making any analysis of the magnetospheric region even more complicated.

- The subsonic settling accretion regime. As mentioned before, it is usually assumed that when Eq. 37 is satisfied, the subsonic propeller is not applicable and all material captured by the neutron star at $R_{\text {acc }}$ can be accreted at the Bondi-Hoyle rate (Eq. 21). Shakura et al. (2012) studied this process in a more detailed way. These authors solved analytically the structure of the spherical shell around a wind-accreting neutron star, assuming that its magnetospheric boundary is RTI unstable (i.e. assuming that Eq. 37 is valid and satisfied). They found that the Bondi-Hoyle rate can only be achieved when the $\mathrm{X}$-ray luminosity from the central neutron star is $\gtrsim 4 \times 10^{36} \mathrm{erg} \mathrm{s}^{-1}$ (for typical values of the systems of interest for this review) and the inflowing material from the companion can be efficiently Compton cooled down already at $R_{\text {acc }}$. At lower luminosities, a subsonic settling accretion regime is achieved. In this regime, the mean radial velocity, $v_{\mathrm{r}}$, of the plasma filling the region between $R_{\mathrm{M}}$ and $R_{\mathrm{acc}}$ is lower than the free-fall velocity $v_{\mathrm{ff}}\left(v_{\mathrm{r}}=f(v) v_{\mathrm{ff}}, f(v)<1\right)$ and depends on the cooling time, $t_{\text {cool }}$, near the magnetosphere, so that $f(v) \sim\left[t_{\mathrm{ff}}\left(R_{\mathrm{acc}}\right) / t_{\text {cool }}\left(R_{\mathrm{acc}}\right)\right]^{1 / 3}$. Two different sub-regimes are identified, depending on the dominant cooling mechanism at the magnetospheric radius. The authors showed that if Compton cooling at the magnetospheric radius dominates over radiative losses, then

$$
f(v) \sim 0.3\left(L_{\mathrm{X}} / 10^{36} \mathrm{erg} \mathrm{s}^{-1}\right)^{-2 / 11} \mu_{30}^{6 / 11},
$$

and thus the mass accretion rate onto the neutron star is reduced by roughly a factor of 3 compared to the Bondi-Hoyle rate. While if radiative cooling dominates, then

$$
f(v) \sim 0.1 \zeta^{14 / 27}\left(L_{\mathrm{X}} / 10^{36} \mathrm{erg} \mathrm{s}^{-1}\right)^{6 / 27} \mu_{30}^{2 / 27}
$$

and the mass accretion rate onto the NS is reduced by a factor of $\sim 10$ compared to the Bondi-Hoyle rate (for typical parameters). In the above equation, $\zeta<1$ is a numerical parameter describing the extension of the transition zone inside and outside the neutron star magnetosphere (Shakura et al., 2013). The transition between the Compton and the radiative cooling regimes is mainly regulated by the neutron star X-ray luminosity. This value of the luminosity can be determined by comparing the cooling time scale in the two cases. As detailed in Shakura et al. (2013), the time scale for the Compton cooling is

$$
t_{\text {Compton }} \simeq 10\left(R_{\mathrm{M}} / 10^{9} \mathrm{~cm}\right)^{2}\left(L_{\mathrm{X}} / 10^{36} \mathrm{erg} \mathrm{s}^{-1}\right)^{-1} \mathrm{~s}
$$

while for the radiative cooling is

$$
t_{\text {radiative }} \simeq 1000\left(R_{\mathrm{M}} / 10^{9} \mathrm{~cm}\right)\left(L_{\mathrm{X}} / 10^{36} \mathrm{erg} \mathrm{s}^{-1}\right)^{-1}(f(v) / 0.3) \mathrm{s}
$$

For typical parameters, it can be seen that Compton cooling dominates for $L_{\mathrm{X}} \gtrsim 3 \times 10^{35} \mathrm{erg} \mathrm{s}^{-1}$.

- The direct accretion regime. At sufficiently high mass inflow rate from the companion star, the material outside the neutron star magnetospheric boundary reaches a critical density above which it is cooled down efficiently for the largest accretion rate to take place (i.e. the Bondi-Hoyle rate in Sect. 2.2.1. According to the calculations in 
Davies and Pringle (1981) and Bozzo et al. (2008), the critical density above which such transition occurs can be written as:

$$
\rho_{\lim _{-12}}=8.3 \times 10^{2} P_{\mathrm{s} 2}^{-3} R_{\mathrm{M} 10}^{5 / 2}\left(1+16 R_{\mathrm{acc} 10} /\left(5 R_{\mathrm{M} 10}\right)\right)^{-3 / 2} .
$$

The accretion of material with the above density would roughly release an X-ray luminosity of $\gtrsim 4 \times 10^{36} \mathrm{erg} \mathrm{s}^{-1}$, that is the transition luminosity estimated by Shakura et al. (2012). In the direct accretion regime, all material captured at $R_{\text {acc }}$ can be readily accreted onto the NS, and the X-ray luminosity released from the system is:

$$
L_{\mathrm{X}}=L_{\mathrm{acc}}=G M_{\mathrm{NS}} \dot{M}_{\mathrm{capt}} / R_{\mathrm{NS}}=5.9 \times 10^{36} v_{8} \rho_{-12} R_{\mathrm{a} 10}^{2} \mathrm{erg} \mathrm{s}^{-1} .
$$

This is the highest X-ray luminosity achievable by a wind-fed neutron star in a $\mathrm{SgXB}$.

Some of the accretion regimes discussed above have been also investigated through numerical simulations, especially the direct accretion regime (Toropin et al., 1999; Toropina et al., 2012) and the supersonic propeller (Romanova et al., 2003). Strongly magnetized neutron stars were also considered (Toropina et al., 2006). The general results obtained within these simulations are qualitatively in agreement with the theoretical predictions discussed above, but a more quantitative comparison would require the usage of full 3D numerical simulations that so far have been developed only for the case of disk accretion and for weakly magnetized neutron stars (see, e.g., Kulkarni and Romanova, 2008; Romanova et al., 2012, and references therein). The issue of extending these simulations to the case of strongly magnetized neutron stars is that for the latter the magnetospheric radius is located at hundreds of stellar radii, thus making the numerical approach used by the simulations challenging.

\subsubsection{X-rays and stellar wind interaction}

In all accretion regimes previously discussed, a conspicuous amount of X-ray radiation is released by the compact object and expected to interact with the surrounding environment. One of the most important effects resulting from this interaction in $\mathrm{SgXBs}$ is the reduction of the radiative force that accelerates the supergiant star wind due to the photoionization of heavy ions. The reduction is strongly related to the value of $L_{X}$ : for sufficiently high X-ray luminosities it is possible that most of the heavy ions in the stellar wind become so largely photoionized that the stellar wind is halted already at the supergiant photosphere, practically inhibiting the formation of a proper stellar wind. The extension of the region in which the wind velocity is affected by the X-ray radiation (measured from the position of the compact object) is called Strömgren sphere. Note that the inhibition of the stellar wind acceleration has a potentially dramatic effect on the accretion process and the emission of the X-ray radiation, as the latter can only be powered when the stellar wind material is channelled on the compact object surface. An elegant analytical approximation describing this feedback mechanism between the wind acceleration and the release of the accretion luminosity was proposed in the seminal paper of Ho and Arons (1987), and thus we summarise here briefly their approach.

It is assumed that the wind of the supergiant star is accelerated according to Eq.7 up to a distance $r_{\xi}$ from the star, where $r_{\xi}$ is the radius of the Strömgren sphere. From this distance onward, the stellar wind moves toward the neutron star with a constant velocity (due to the lack of any efficient acceleration) according to the equation:

$$
v_{\mathrm{w}}= \begin{cases}v_{\infty}\left(1-R_{*} / r\right)^{\beta} & \text { if } r<r_{\xi} \\ v_{\infty}\left(1-R_{*} / r_{\xi}\right)^{\beta} & \text { if } r \geq r_{\xi} .\end{cases}
$$


Here $r$ is the distance from the supergiant star, and $r_{\xi}$ is defined by the equation

$$
\frac{L_{\mathrm{X}}}{n\left(r_{\xi}\right)\left(r-r_{\xi}\right)^{2}}=\xi_{\mathrm{cr}}
$$

If we assume that $n(r) \simeq \rho_{\mathrm{w}}(r) / m_{\mathrm{H}}, \rho_{\mathrm{w}}(r)$ is given by Eq. 20, $L_{\mathrm{X}}$ is given by Eq. 22, $m_{\mathrm{H}}$ is the proton mass, and $\xi_{\text {cr }}$ is the critical value of the so-called ionization parameter $\xi$ at which the wind acceleration is completely cut-off by the X-ray ionization (i.e. the stellar wind becomes virtually transparent to UV photons). The value of $\xi_{\text {cr }}$ is strongly dependent on the chemical composition of the stellar wind and on the details of the micro-physics intervening in the ionization process. Ho and Arons (1987) assumed $\xi_{\mathrm{cr}} \sim 10^{4}$, but such value was then revised to $\xi_{\mathrm{cr}} \sim 3 \times 10^{2}$ by Stevens (1991). By putting together Eq. 47,22, and 20, we obtain

$$
\xi_{c r}=4 \pi m_{\mathrm{H}} \frac{G^{3} M_{\mathrm{NS}}^{3}}{R_{\mathrm{NS}}} \frac{1}{a^{2}\left(\frac{a}{r_{\xi}}-1\right)^{2}} \frac{1}{v_{\mathrm{w}}^{3}\left(r_{\xi}\right)} .
$$

This is the so-called feedback equation of Ho and Arons (1987) and can be solved to derive the distance $r_{\xi}$ from the NS at which its X-ray radiation is able to stop the acceleration of the companion star wind, in turn regulating the mass accretion rate onto the compact object and the production of the X-ray emission itself. Ho and Arons (1987) showed that Eq. 48 has typically two solutions: the first one corresponds to the case in which $r_{\xi} \simeq R_{*}$ and the wind velocity is very much reduced compared to that predicted by the CAK model. This is called the high luminosity solution because for such low velocity Eq. 14 and 22 predict a large X-ray luminosity. In the low luminosity solution, $r_{\xi} \simeq a$ and the expected X-ray luminosity is much lower due to the higher wind velocity (the wind can be accelerated up to the neutron star location). Originally, the presence of these two branches of solutions was used to explain the differences between brighter (Cyg X-1, SMC X-1) and fainter HMXBs (Vela X-1, 4U 1700-37, 4U 1538-52). More recently, their applicability to the case of wind accreting HMXBs was revised and discussed by Karino (2014), who also pointed out the limitations of one dimensional calculations in the case of binary systems. Ducci et al. (2010) also presented an extension of the previous Ho and Arons (1987) calculations, by including in the feedback equation a term describing the reduction in the mass accretion rate onto the neutron star surface caused by the wind material that is not sufficiently accelerated after the ionization to escape the supergiant companion and to reach the compact object.

The convincing observational evidences of the disruption of stellar winds in $\mathrm{SgXBs}$ (see, e.g. Watanabe et al., 2006) provided the required momentum to stimulate refined models of the interaction between the X-rays and the surrounding medium in these systems. Starting from the pioneering paper of Blondin and Woo (1995), in which the first two dimensional calculations of the stellar wind photoionization in HMXBs were presented, a number of recent studies were presented which include a more realistic description of the stellar wind composition and the photoionization process. We discuss the results of these papers in more details in Sect. 4.

We note that a different way to probe the interaction between the X-rays from the neutron star and the surrounding stellar wind is offered by the so-called HM-effect (Hatchett and McCray, 1977). The presence of the Strömgren sphere around the compact object was demonstrated to induce noticeable variations in the UV resonance lines produced within the massive star wind and providing measurements of the local wind velocity. When applied to the case of Vela X-1, the results of detailed spectroscopic observations obtained in the UV domain allow us to compare the properties of the photoionized region around the neutron star with 
that determined from complementary investigations in the X-ray domain van Loon et al., 2001; Watanabe et al., 2006). The major limitation impacting the exploitation of the HMeffect to study photoionization in wind-fed binaries is the availability of high resolution UV spectra of these systems with a sufficiently high signal-to-noise ratio. So far the HM-effect could be verified only in the cases of Vela X-1 and $4 \mathrm{U} 1700-37$, as these are the closest-by and brightest wind-fed SgXBs. For all other known sources of the same class, no data of the required quality could be obtained so far in the UV domain and thus the applicability of the HM-effect as remained largely unexplored (see van Loon et al., 2001, and references therein).

\section{Stellar and wind parameters of massive stars}

\subsection{Quantitative spectroscopy}

Stellar and wind-parameters of hot massive stars are conventionally determined by means of quantitative spectroscopy, i.e., by fitting synthetic spectral energy distributions and normalized spectre 20 to observations, mostly in the optical and/or the UV range. Meaningful mass-loss diagnostics are also provided by observations in the X-ray (see Sect. 3.8) and far-IR/mm/radio domain.

For calculating synthetic energy distributions, a consistent treatment of photosphere and wind requires so-called unified model atmospheres, at least if the lines/continua are formed outside the (quasi-) hydrostatic region. Such unified model atmospheres have been introduced by Gabler et al. (1989). By means of a typical velocity law (with $\beta=1$, see Eq. 7), one can derive a maximum $\dot{M}$ for which a hydrostatic treatment is still possible, at least in the optical and most of the UV. Estimating a limiting Rosseland optical depth $\tau_{\text {Ross }}<10^{-2}$ at the transition between the photosphere and wind (roughly located at $10 \%$ of the sound-speed), one finds $\dot{M}<6 \cdot 10^{-8} M_{\odot} \mathrm{yr}^{-1}\left(R_{*} / 10 R_{\odot}\right)\left(v_{\infty} / 1000 \mathrm{~km} \mathrm{~s}^{-1}\right)$. Comparing with "observed" (i.e., empirically derived) mass-loss rates, this limit implies that hydrostatic models are still largely sufficient for late O-dwarfs, B-stars up to luminosity class II (for early sub-types) or Ib (for mid/late sub-types), and A-stars up to luminosity class Ib. Above this limit, unified model atmospheres have to be used, since the spectral appearance is considerably affected by wind effects, even if there are no apparent wind features in a particular observation. Note that unified model atmospheres are computationally expensive, because of the coupling between velocities and frequencies via corresponding Doppler-shifts. Moreover, all models, even the purely hydrostatic ones, need to account for NLTE conditions, due to the high temperatures and comparatively low densities.

Table 1 compares present state-of-the-art atmospheric codes which can be used for the spectroscopic analysis of hot stars. Since the codes Detail/surface and TLUSTY calculate occupation numbers/spectra on top of hydrostatic, plane-parallel atmospheres, they are "only" suited for the analysis of stars with negligible winds (see above). The different computation times are mainly caused by the different approaches to deal with line-blocking/ blanketing. The overall agreement between the various codes (within their domain of application) is quite satisfactory, though certain discrepancies are found in specific parameter ranges, particularly regarding EUV ionizing fluxes (Puls et al., 2005; ; Simón-Díaz and Stasińska, 2008) and surface gravities (Massey et al., 2013).

\footnotetext{
20 Colours are less meaningful (or not usable at all), because of the high temperatures.
} 
Table 1 Comparison of state-of-the-art, NLTE, line-blanketed model atmosphere codes suited for the analysis of hot massive stars

\begin{tabular}{|c|c|c|c|c|c|c|c|}
\hline code & $\begin{array}{l}\text { Detail/ } \\
\text { Surface }^{1}\end{array}$ & TLUSTY $^{2}$ & PoWR ${ }^{3}$ & PHOENIX $^{4}$ & CMFGEN $^{5}$ & WM-basic $^{6}$ & FASTWIND $^{7}$ \\
\hline geometry & $\begin{array}{l}\text { plane- } \\
\text { parallel }\end{array}$ & $\begin{array}{l}\text { plane- } \\
\text { parallel }\end{array}$ & spherical & $\begin{array}{l}\text { spherical/ } \\
\text { pl.-parallel }\end{array}$ & spherical & spherical & spherical \\
\hline blanketing & LTE & yes & yes & yes & yes & yes & approx. \\
\hline $\begin{array}{l}\text { diagnostic } \\
\text { range }\end{array}$ & $\begin{array}{l}\text { no } \\
\text { limitations }\end{array}$ & $\begin{array}{l}\text { no } \\
\text { limitations }\end{array}$ & $\begin{array}{l}\text { no } \\
\text { limitations }\end{array}$ & $\begin{array}{l}\text { no } \\
\text { limitations }\end{array}$ & $\begin{array}{l}\text { no } \\
\text { limitations }\end{array}$ & UV & optical/IR \\
\hline $\begin{array}{l}\text { major } \\
\text { application }\end{array}$ & $\begin{array}{l}\text { BA stars } \\
\text { with negl. } \\
\text { winds }\end{array}$ & $\begin{array}{l}\text { hot stars } \\
\text { with negl. } \\
\text { winds }\end{array}$ & $\begin{array}{l}\text { WRs, } \\
\text { OB-stars }\end{array}$ & $\begin{array}{l}\text { cool stars, } \\
\text { SNe }\end{array}$ & $\begin{array}{l}\mathrm{OB}(\mathrm{A})- \\
\text { stars, } \\
\text { WRs, SNe }\end{array}$ & $\begin{array}{l}\text { hot stars w. } \\
\text { dense winds, } \\
\text { SNe }\end{array}$ & $\begin{array}{l}\text { OB-stars, } \\
\text { early A-sgs }\end{array}$ \\
\hline comments & no wind & no wind & - & $\begin{array}{l}\text { no clumping } \\
\text { no X-rays }\end{array}$ & - & no clumping & $\begin{array}{l}\text { no X-rays } \\
\text { (in progress) }\end{array}$ \\
\hline $\begin{array}{l}\text { execution } \\
\text { time }\end{array}$ & $\begin{array}{l}\text { few } \\
\text { minutes }\end{array}$ & hours & hours & hours & hours & 1 to $2 \mathrm{~h}$ & $\begin{array}{l}\text { few min. } \\
\text { to } 0.5 \mathrm{~h}\end{array}$ \\
\hline
\end{tabular}

(1) Giddings (1981); Butler and Giddings (1985), (2) Hubeny (1998), (3) Gräfener et al. (2002), (4) Hauschildt (1992), (5) Hillier and Miller (1998), (6) Pauldrach et al. (2001), (7) Puls et al. (2005)

The best fitting model-spectra and thus the corresponding stellar and wind parameters are found by either a simple 'by-eye'-inspection (e.g., Repolust et al. 2004), or by minimization methods (using a genetic algorithm, e.g., Mokiem et al. 2005, or pre-calculated model-grids, e.g., Lanz and Hubenv 2003; Hamann and Gräfener 2004; Lefever et al. 2010; Simón-Díaz et al. 2011; Sander et al. 2012; Hainich et al. 2014). It should be noted that a proper comparison between observed and model spectra can only be automatized to a certain degree as it requires detailed knowledge about which features are affected by which parameters. Furthermore, such comparisons should be made with as many lines as possible, as a particular single line can be affected by a variety of parameters which can lead, in different combinations, to the same line shape.

\subsection{Stellar parameters}

Regarding the individual stellar parameters, the following prime diagnostics are available: effective temperatures, $T_{\text {eff }}$, are derived from the ionization equilibrium 21 of nitrogen $(\mathrm{O} 2$, $\mathrm{O} 3$, and $\mathrm{WN}$ ), helium (WR and O-stars up to O4), silicon (B-stars), and magnesium (Astars). Typical accuracies are 5\%. Surface gravities are derived from the wings of the Starkbroadened Balmer lines $( \pm 0.1 \mathrm{dex})$. This method works only for purely photospheric absorption lines, i.e., $\mathrm{H}_{\gamma}$ and/or $\mathrm{H}_{\delta}$. Thus, if the available spectral range consists of emission lines only, there is no direct spectral diagnostics to infer the effective surface gravity. Radii and thus luminosities are obtained from comparing the theoretical and absolute observed fluxes 22 , or from using $T_{\text {eff }}, M_{V}$ and the bolometric correction. The distance is the major source of error here. The abundances of individual elements are finally determined from fits to their corresponding spectral lines (in the optical and the UV), where, if possible, photospheric lines should be used, to avoid contamination from wind-inhomogeneities and windembedded shocks. Corresponding errors depend on the complexity of the line-formation

\footnotetext{
21 By fitting the corresponding photospheric lines/line-strength ratios from two (or more) neighbouring ionization stages

22 If this is done over a wider range, the term spectral energy distribution (SED) is typically used.
} 
and on the number of available diagnostic lines. E.g., for BA-stars very accurate measurements of the abundances are possible for many elements ( 0.05 to 0.1 dex, see Przybilla et al. 2008), whilst for nitrogen and carbon in O-stars the typical errors are considerably larger ( 0.15 to 0.2 dex or higher), due to the complex line-formation (Rivero González et al., 2012; Martins and Hillier, 2012), and the scarcity of lines. Finally, we note that a complete analysis involves many more parameters, such as projected rotational velocity, $v \sin i$, micro- and macro-turbulence. A discussion of these parameters is beyond the scope of this review, just to mention that the measurement of $v \sin i$ is quite precise if the rotational speed exceeds $100 \mathrm{~km} \mathrm{~s}^{-1}$, and it becomes problematic for speeds below $40 \mathrm{~km} \mathrm{~s}^{-1}$ (e.g., Sundqvist et al. 2013).

\subsection{Velocity field}

Since the measurement of $\dot{M}$, one of the most important parameters in this context, will be detailed in the next section, we will concentrate here on the velocity field. Terminal velocities $v_{\infty}$ can be quite easily measured from the blue edge 23 of UV P Cygni profiles (typical accuracy: 10\%), as long as these profiles display strong absorption (and the UV has been observed!). Weaker resonance lines (e.g., from late $\mathrm{O}$ and B-dwarfs) pose a severe problem in this regard, since it is quite likely that the apparent edge velocity is lower than the actual $v_{\infty}$. When possible, the asymptotic behaviour of DACs (Sect.2.1.3) can be utilized here. In many cases, additional spectral modelling is necessary to confirm velocity measurements from UV resonance lines, since those lines are located in the iron forest which often dominates the spectral appearance in the UV. If, however, the UV has not been observed, scaling relations derived from a multitude of measurements can be used (e.g., Kudritzki and Puls 2000), or, in the case of mid/late B- and A-supergiants, $v_{\infty}$ might be derived from $\mathrm{H}_{\alpha}$ Kudritzki et al., 1999) which displays a P Cygni profile for these spectral types. Finally, the slope of the velocity law (usually corresponding to the value of $\beta 24$ ) can be determined from the shape of the UV P Cygni emission and/or the shape of $\mathrm{H}_{\alpha} / \mathrm{He}$ II 4686, if in emission. We note, however, that particularly the latter optical diagnostics also depend on the degree and stratification of the wind clumping, thus introducing a significant dichotomy. Since a particular line can easily be affected by a variety of phenomena, we stress again that all diagnostics should not rely on a single line alone, but needs to be performed in combination with a reasonable fit to the overall available spectrum. Most unified model atmospheres are applicable for a wide spectral range (cf. Table 1), and the better the overall reproduction of the observed spectrum is, the less is the chance to mis- or over-interpret a particular feature.

\subsection{Mass-loss diagnostics and the effects of wind clumping}

The most widely used diagnostics to determine mass-loss rates from $\mathrm{O}$ and WR stars are: $i$ ) thermal radio continuum emission; ii) ultraviolet resonance lines, and iii) UV and optical emission lines (see Lamers and Cassinelli (1999) for details).

Besides these well established methods, other empiric diagnostics of mass-loss rates have been suggested. E.g., Cohen et al. (2010a) proposed to measure mass-loss rates by analysing X-ray emission line profiles. Prinja and Massa (2013) suggested that the ratio of

\footnotetext{
23 Or from the black troughs, if present

24 Sometimes, two velocity laws with different $\beta$ are combined, e.g. Todt et al. 2010
} 
narrow features observed at terminal velocities in the UV resonance lines of B-supergiants provides a clumping independent tool to estimate mass-loss rates in these objects. This may be especially important because in B-type supergiants, the $\mathrm{H} \alpha$ line might be optically thick, and thus lose its diagnostic value (Petrov et al., 2014). Kobulnicky et al. (2010) suggested that IR observations of the circumstellar medium around massive stars provide a new laboratory for estimating stellar mass loss rates. This idea was applied in Hubrig et al. (2011) and further developed in Gvaramadze et al. (2012). Huenemoerder et al. (2012) suggested that the X-ray flux may provide a reliable mass-loss diagnostics in late type O-dwarfs. All this is by far not an exhaustive list of possible mass-loss diagnostics, but below we will concentrate on already well established methods.

The thermal radio emission is easy to interpret (Wright and Barlow, 1975), but it can only be observed in a small number of nearby stars. The other two diagnostics are based on spectroscopic models, and their interpretation is not straightforward. Many investigations are restricted to a semi-empirical modelling of the line profiles, e.g. using the "Sobolev with exact integration method" (SEI, Hamann, 1981; Lamers et al., 1987) and a subsequent interpretation of the derived optical-depth parameters. Not being based on consistent models, the obtained mass-loss rates are highly uncertain (e.g. because of the unknown degrees of ionization). Moreover, most resonance lines in the UV range (e.g. N v, C IV) are often saturated and therefore not suitable for precise $\dot{M}$ determinations. The far-UV (FUV) includes the P V resonance line which is very useful because it is unsaturated in typical O stars (Bouret et al., 2005; Fullerton et al., 2006), due to the low phosphorus abundance. However, since only a few instruments - such as the FUSE satellite - have been capable to observe in the FUV range, such data are rare.

A quantitative analysis of the emissior 25 lines (e.g. $\mathrm{H} \alpha$ ), as well as a reliable interpretation of the UV resonance lines, requires a detailed modelling of the stellar atmosphere and wind. The conditions in the supersonic winds from hot stars deviate extremely from local thermodynamical equilibrium, therefore the spectral analysis has to be conducted by means of appropriate stellar atmosphere models (see Table 1 ). The advances in the radiative transfer offered by these NLTE codes are huge, however, the geometries that are adopted are rather simple. Typically, only spherically symmetric stellar winds are described by the NLTE stellar atmospheres. Consequently, wind clumping can be included in these sophisticated codes only using some approximations.

\subsection{Microclumping}

The most stringent possible approximation is that clumping does not directly 26 affect the radiative transfer in a stellar wind. This would be the case when wind clumps are assumed to be optically thin at all frequencies. This approximation is called "microclumping" (or optically thin clumping) and was implemented in such codes as PoWR and CMFGEN already back in the 1990s.

It is assumed that inside clumps the density is uniform, and enhanced by a factor $D$ compared to a smooth model with the same mass-loss rate $\dot{M}$. The volume filling factor of the clumps is $f_{\mathrm{V}}=D^{-1}$, because the interclump medium is assumed to be void. Thanks to the latter assumption, the rate equations have to be solved only for the clump medium, i.e. for the enhanced density $\rho_{\mathrm{C}}=D \rho$. From the obtained population numbers, the non-LTE opacity and emissivity of the clump matter, $\kappa_{C}(D \rho)$ and $\eta_{C}(D \rho)$, can be calculated.

\footnotetext{
25 for low wind-densities, the wind emission 'only' fills in the photospheric absorption
}

26 but note that, e.g., optical depths can change, see below 
In the radiative transfer equation, the smooth-wind opacity and emissivity $\kappa(\rho)$ and $\eta(\rho)$ must be replaced for a clumped wind by

$$
\kappa_{\mathrm{f}}=f_{\mathrm{V}} \kappa_{\mathrm{C}}(D \rho) \text { and } \eta_{\mathrm{f}}=f_{\mathrm{V}} \eta_{\mathrm{C}}(D \rho) \text {. }
$$

The atomic transition rates contributing to the opacity and emissivity scale with different powers of the density depending on their respective nature. For processes linear in density, $f_{\mathrm{V}}$ and $D$ cancel, but contributions scaling with the square of the density (bound-free emission, or free-free absorption and emission) are effectively enhanced by a factor of $D$, which may depend on the location in the stellar wind, i.e. on the radial coordinate $r$.

Empirical mass-loss diagnostics are widely based on processes that scale with the square of the density. The wind emission lines in Wolf-Rayet and $\mathrm{O}$ stars, including $\mathrm{H} \alpha$, form in de-excitation cascades that are fed by radiative recombination. The thermal radio emission from stellar winds is due to the free-free process. Consequently, when a given (radio or line) emission is analysed with a model that accounts for microclumping, the derived mass-loss rate will be lower by a factor of $\sqrt{D}$ relative to a smooth-wind model.

Fitting the observed electron scattering wings of strong emission lines in Wolf-Rayet spectra can be used to determine the clumping factor $D$ and its radial dependence (Hillier, 1991; Hamann and Koesterke, 1998). For WR stars this method yields typical clumping factors $D$ between 4 and 10 (e.g., Hamann and Koesterke, 1998). Unfortunately, the method is not applicable to $\mathrm{O}$ stars, since their spectra do not show suitable emission lines. In this case, only the resonance lines remain as a density-linear diagnostic among the mass loss indicators, especially the unsaturated $\mathrm{P} V$ doublet.

For these stars too, the mass-loss rate derived from smooth models and density-squared diagnostics ( $\mathrm{H} \alpha$, infra-red and radio emission) needs to be scaled down by the square root of the clumping factor.

Strong clumping and correspondingly low mass-loss rates were obtained by Bouret et al. (2005) for two O stars analysed with non-LTE model atmospheres. Fullerton et al. (2006) exploited the fact that the far-UV spectral range observed with FUSE contains the P V resonance line, which is typically unsaturated in O-star spectra, and analysed this line in spectra of 40 O-type stars with the SEI method. They found a large discrepancy between mass-loss rates obtained from $\rho^{2}$ diagnostics (such as the analysis of $\mathrm{H} \alpha$ line or radio emission) based on unclumped models and mass-loss rates from PV lines. To overcome this discordance it was suggested that the clumping is very strong $\left(f_{\mathrm{V}} \lesssim 0.01\right)$ and therefore mass-loss rates have to be reduced by orders of magnitude.

The drastic revisions of stellar mass-loss rates because of clumping suggested from FUV diagnostics were in apparent agreement with insights gained from the analysis of high-resolution X-ray spectra of O-stars. According to the widely accepted scenario (e.g., Feldmeier et al. 1997a, see Sect. 2.1.2), X-rays from hot-star winds are produced in shockheated gas, while the bulk of the stellar wind is cool and absorbs part of the X-rays before they can emerge. The X-rays are mainly emitted in spectral lines, while the absorption is continuous (K-shell photoionization). This decoupling of emission and absorption greatly simplifies modelling of the transfer of X-rays in stellar wind. The shape of emission line profiles for such a situation was predicted by Macfarlane et al. (1991).

Macfarlane et al. (1991) considered the optically thin emission from a radially expanding shell of hot gas which suffers absorption in the smooth cool stellar wind. When this absorption is small, the line is broad and has a box-like shape. For stronger wind absorption, the line becomes more skewed (see Fig. 7 in Macfarlane et al. (1991)). The optical depth along the radial line of sight is given by $\tau_{\lambda}=\int \chi_{\lambda}(r) \mathrm{d} r$, where the atomic opacity $\left(\chi_{\lambda}=\rho_{\mathrm{w}} \kappa_{\lambda}\right)$ 
is the product of the mass absorption coefficient $\left(\kappa_{\lambda}\left[\mathrm{cm}^{2} \mathrm{~g}^{-1}\right]\right)$, and the density of the cool wind $\left(\rho_{\mathrm{w}}\right)$. The latter is obtained from the continuity equation $\dot{M}=4 \pi \rho_{\mathrm{w}}(r) v(r) r^{2} R_{*}^{2}$, where $r$ is the radial distance in units of $R_{*}$, and $v(r)$ is the velocity law that is prescribed by the expression (see also Eq. 7) $v(r)=v_{\infty}(1-1 / r)^{\beta} \equiv v_{\infty} w(r)$. Neglecting the radial dependence of the mass absorption coefficient, the optical depth can be parameterized as $\tau_{\lambda}=\tau_{0} \int w(r)^{-1} r^{-2} \mathrm{~d} r$, where

$$
\tau_{0}=\frac{\kappa_{\lambda} \dot{M}}{4 \pi v_{\infty} R_{*}}
$$

Assuming that the visible emission most likely originates far from the stellar core in the wind regions with constant velocity, Macfarlane et al. (1991) adopted a constant velocity $\left(v_{\infty}\right)$. The line shape is largely determined by the parameter $\tau_{0}$. Macfarlane et al. (1991) noticed that when $\tau_{0}$ increases, the red-shifted part of the line $(\Delta \lambda>0)$ becomes significantly more attenuated than the blue-shifted part (see Fig. 3). They suggested that evaluating the line shape can be used to determine $\tau_{0}$ and the wind density.

The K-shell opacities vary with wavelength with a power between 2 and 3 (e.g., Verner et al. 1996). Therefore $\tau_{0}$ should change by orders of magnitude in the X-ray band. Consequently, the X-ray emission line shape at shorter and longer wavelengths should be different.

The follow-up works by Ignace (2001) and Owocki and Cohen (2001) applied the Macfarlane et al. (1991) results also for X-ray emission lines, where the latter improved upon Macfarlane et al. (1991)'s model by, e.g., assuming a spatially distributed emitting plasma with velocity kinematics (used also to calculate the cool wind density) according to the "beta-law" given above (which can lead to quite different line-profiles than when assuming emission only at a specific location).

The first high-resolution X-ray spectrum of an O star was obtained by Waldron and Cassinelli (2001). Their analysis of the MEG spectrum of the O9.7Ib star $\zeta$ Ori revealed that the hot plasma is located relatively close to the stellar photosphere and that the line profiles are nearly symmetric and not skewed. Waldron and Cassinelli (2001) expanded the singleshock model of Macfarlane et al. (1991) and considered emission from spherically symmetric shocks equally distributed between $0.4 v_{\infty}$ and $0.97 v_{\infty}$ with temperatures ranging from 2 to $10 \mathrm{MK}$. They found that to explain the nearly symmetric X-ray emission line profiles, the mass-loss rate from the O-type supergiant $\zeta$ Ori must be an order of magnitude lower than derived from radio and UV-diagnostics (Lamers and Morton, 1976; Abbott et al., 1980).

Similarly, much less skewed than expected X-ray line profiles were measured from other O-type stars. Fitting observed lines with the Owocki and Cohen (2001) model generally yields quite low values of $\tau_{0}$, with weaker dependence on wavelength than expected from a smooth wind with corresponding mass-loss rates derived from, e.g., $\mathrm{H} \alpha$ using smooth wind models (Cassinelli et al., 2001; Kahn et al., 2001; Kramer et al., 2003). One possible explanation of these results is a thinner wind than predicted by "standard" theoretical wind models like Vink et al. (2000).

\subsection{New evolutionary scenario for massive stars?}

Thus, about a decade ago the massive star community encountered a severe problem - based on UV and X-ray diagnostics, the empirically estimated mass-loss rates from O-type stars were significantly lower than predicted by the theory. These low mass-loss rates were the basis for Smith and Owocki (2006) to conclude that the "reduced mass-loss rates mean that steady winds are simply inadequate for the envelope shedding needed to form a WR star". Hence, they proposed a new scenario according to which the stars with initial mass above 


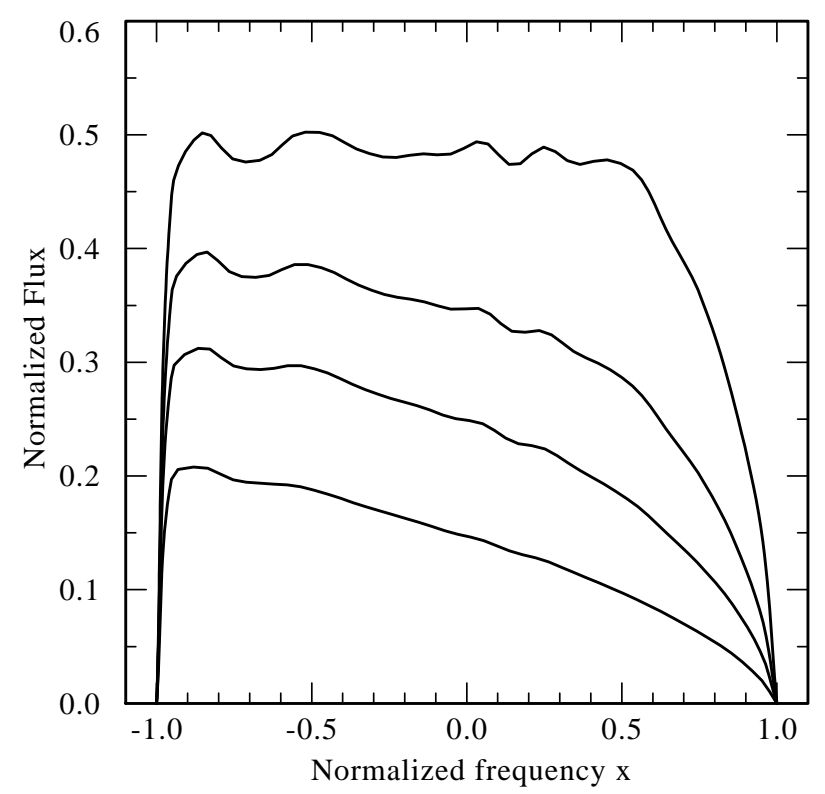

Fig. 3 The X-ray emission line profiles based on Macfarlane et al. (1991) assuming a smooth wind with constant wind velocity. For convenience we display the computed line profiles as a function of the dimensionless frequency $x$, measured relative to the line centre and in Doppler units referring to the terminal wind velocity. The negative $x$ refers to blue frequencies. From the top to the bottom line profile, $\kappa_{\lambda}=1,10,20,40$. Occultation by the stellar core is included in the model. The lines are not smooth because the X-ray emission is assumed to originate from a limited number of small parcels of hot gas randomly distributed in the wind (see details in Oskinova et al. (2004)).

$40 \ldots 50 M_{\odot}$ loose the bulk of their mass not via radiation line-driven stellar winds, but via optically thick, continuum-driven outbursts (or by hydrodynamic explosions). It was also suggested that Wolf-Rayet type stars may not be the descendants of the most massive stars, with the latter exploding as SNe at the LBV evolutionary stage.

This far reaching scenario challenged stellar evolution calculations (see also Groh et al., 2014) but was largely grounded on the empirical mass-loss rates derived using the assumption of microclumping.

\subsection{Macroclumping}

While the microclumping approximation is extremely convenient it was always clear that this approach is too stringent to describe realistic winds.

Massa et al. (2003) and Fullerton et al. (2006) realized that for strong lines in dense winds microclumping is not justified. They discussed that correctly accounting for clumping in UV resonance lines can affect the formation of P Cygni lines and, in extreme cases, produce an apparently unsaturated profile for a line that would be extremely saturated if the wind material were distributed smoothly. Prinja and Massa (2010) studied B-supergiant winds and found wide-spread signatures of optically thick clumping in spectra of these stars.

Oskinova et al. (2007) demonstrated that the microclumping approximation is not suitable for empirical mass-loss diagnostics based on UV resonance lines formed in massive star winds. They developed a radiative transfer technique that describes realistic stellar 
winds without relying on the microclumping approximation. This approach, which is called "macroclumping" (using the physical analogy with micro- and macroturbulence) was successfully used to demonstrate the concordance of empirical mass-loss rates derived from $\rho$ and $\rho^{2}$ diagnostics.

It was shown that while microclumping is not suitable for UV-resonance lines, it still can be used for the $\mathrm{H} \alpha$-line in O-supergiants. As a result, the mass-loss rate should be reduced by a factor $\sqrt{D}$ (see Eq.49) compared to the unclumped models. In case of $\zeta$ Puppis, a satisfactory fit to $\mathrm{H} \alpha$ was produced with $D=10$. Consequently, the mass-loss rate was shown to be lower by a factor 2-3 than in unclumped models. Independent work by other authors (see below) largely confirmed this approach also in other O-supergiants.

Waiving the microclumping approximation in modelling UV resonance lines requires to account for clumps of arbitrary optical thickness in line radiative transfer. For radiative transfer in lines, a clump opacity depends on its geometry, abundances, and velocity field. The latter is because with lower velocity dispersion within a clump, the Doppler broadening is smaller, and the line absorption profile is narrower but peaks higher. Thus, the clump optical depth in the line core becomes larger, while it is smaller in the line wings. Higher optical depth in the line core leads to a reduction of the effective opacity and a weakening of the line (see Fig. 5 in Oskinova et al., 2007). In a supersonically expanding medium, rays of a given (observer's frame) frequency can only interact with clumps near the surface of constant radial velocity (CRVS). The line opacity of the other clumps is Doppler-shifted out of the resonance. The porosity effect for lines is very pronounced (Hamann et al., 2008), and this porosity stems from the fact that the clumps are not covering the complete velocity field, rather than from clumps that are spread out spatially in the wind. As such, other authors (Owocki, 2008; Sundqvist et al., 2010, 2014) explicitly differentiate between porosity in physical and velocity space.

To implement the macroclumping approach in PoWR NLTE model atmospheres, a onecomponent wind model was considered, where the wind consists only of clumps (assumed to follow the " $\beta$-law" velocity law, see above) and is void between them. Moreover, the clumps were treated statistically, with two key parameters describing clumped wind properties, $L_{0}$ and $v_{\mathrm{D}}$, where the former is a parameter setting the average separation between clumps in the wind, and the latter describes the velocity distribution within clumps. The macroclumping formalism was implemented in the formal solution of the radiative transfer equation. The synthetic spectra were compared to the observed, and it was shown that mass-loss rates inferred from optically thin emission, such as the $\mathrm{H} \alpha$ line in $\mathrm{O}$ stars, are not influenced by macroclumping. On the other hand, the strength of optically thick resonance lines, such as P V, was strongly reduced because of macroclumping effects. Thus it was demonstrated that the microclumping approximation is not automatically valid for resonance lines. Consequently, relying on this approximation can lead to underestimating empirical mass-loss rates.

The shortcomings of the statistical approach used in Oskinova et al. (2007) were overcome by Monte Carlo radiative transfer models for inhomogeneous expanding stellar winds (Šurlan et al., 2012). While still relying on a phenomenological description of the wind (i.e. the hydro-equations are not being solved), this code provided a full 3-D description of the dense as well as the tenuous wind components enabling the modelling of resonance lines in a clumped stellar wind. The code also accounts for non-monotonic velocity fields.

Šurlan et al. (2013) analysed the observed optical and UV spectra of O-type stars. As a first step, the observed spectra were modeled with PoWR model atmospheres, and the empirical mass-loss rates were estimated from fitting the observed $\mathrm{H} \alpha$ emission lines. As a next step, the unsaturated UV resonance lines (i.e., P v) were modeled using a 3D Monte- 


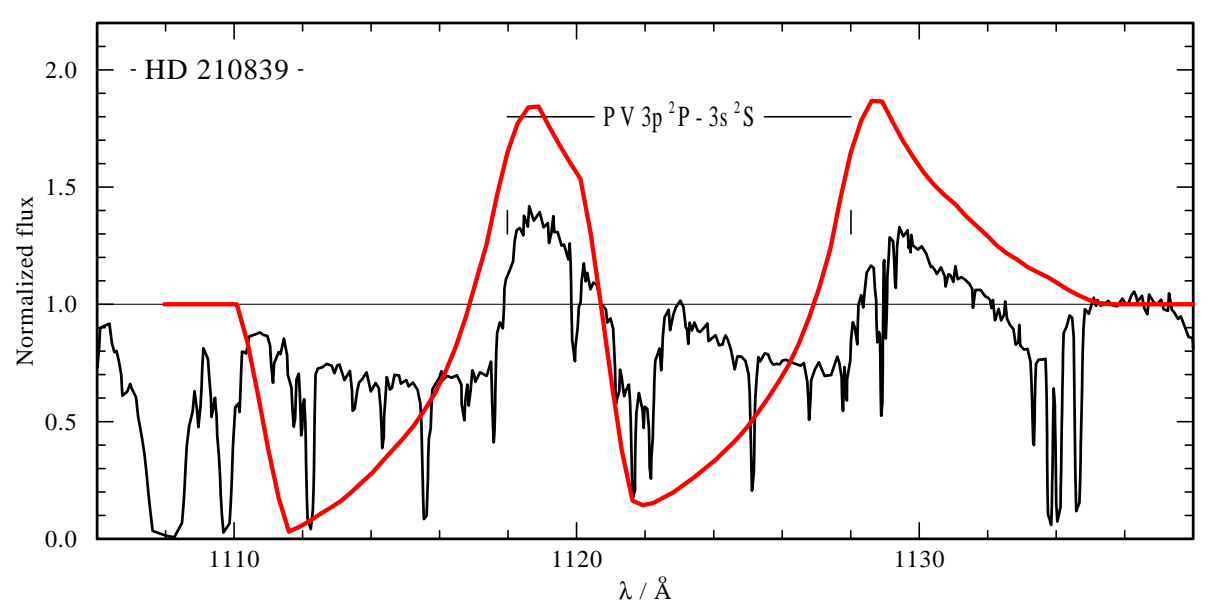

Fig. 4 Comparison of the observed PV doublet in the UV spectrum of $\lambda$ Cep (thin black line) and the model line calculated for $\dot{M}=1.6 \times 10^{-6} M_{\odot} \mathrm{yr}^{-1}$ using the microclumping approximation (figure courtesy B. Kubatova)

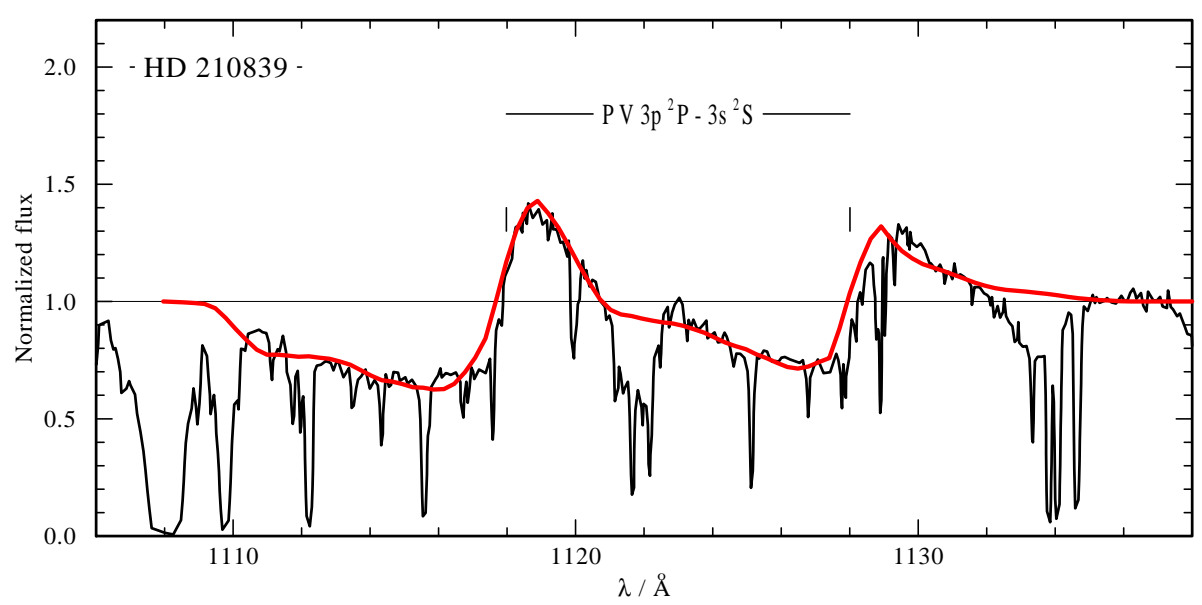

Fig. 5 The same as in Fig. 4 except that the model that is computed is based on a 3-D macroclumping wind model with the same mass-loss rate $\dot{M}=1.6 \times 10^{-6} M_{\odot} \mathrm{yr}^{-1}$ (figure courtesy B. Kubatova)

Carlo code. The ionization stratification and underlying photospheric spectra were adopted from the PoWR models. This allowed to constrain the properties of the wind clumps by fitting the observed resonance line profiles. It was shown that the UV resonance lines, such as the unsaturated doublet of $\mathrm{PV}$, can be easily reproduced when macroclumping is taken into account.

Including a velocity dispersion within the clumps helps to improve the fitting of the line wings and, thus, allows a better agreement with the observations.

To illustrate the effects of macroclumping, in Fig. 4 we compare the $\mathrm{P} v$ line observed in the O-type star $\lambda$ Cep and the model spectrum with $\dot{M}=1.6 \times 10^{-6} M_{\odot} \mathrm{yr}^{-1}$ of the same line computed with a standard model and a microclumping approximation. Clearly, the model 
severely over-predicts the line strength. In order to match the observed and the model lines using the microclumping approximation one would need to significantly reduce the massloss rate. Alternatively, the abundance of phosphorus could be severely reduced as well as some fitting parameters for the radial distribution of the volume filling factor could be introduced (e.g., Bouret et al., 2012). However, Figure 5 illustrates that these problems are easily overcome when the microclumping approximation is waived, and realistic 3-D macroclumping models (Šurlan et al., 2012) are used.

Independent from the work by Šurlan et al. (2012, 2013), Sundqvist and collaborators, in a series of publications (Sundqvist et al., 2010, 2011, 2014), solved the problem in a semi-analytic way, providing expressions that can be implemented in present-day NLTE codes, thus accounting for optically thick clumping not only in the formal solution, but also in the NLTE rate equations. Thus, they provide a tool that accounts for the back-reaction of such clumping onto the ionization balance and excitation, and which has been benchmarked using multi-D models (e.g., Sundqvist et al., 2011) similar to those described above by Šurlan et al. (2012, 2013).

Another independent study was performed by Muijres et al. (2011) and collaborators who combined non-LTE model atmospheres and a Monte Carlo method to compute the transfer of momentum from the photons to the gas in an atmosphere with clumping and porosity. Effects of clumping at the photosphere were also considered. It was shown that the line force increases as a result of increased recombination in a clumped wind. However, the line force decreases because of porosity (or macroclumping), simply because photons may travel in between the clumps, avoiding interactions with the gas. The effects of clump size on the mass-loss rate were demonstrated, e.g. it was shown that different predicted massloss rates are expected for different clump sizes. E.g. very high overdensities of gas in large clumps may result in the predicted $\dot{M}$ to decrease by factors 10 to 100 compared to winds with less dense and smaller clumps.

Recently, Noebauer and Sim (2015) have introduced a new approach to solve line-driven stellar winds self-consistently by using a Monte Carlo-based radiation hydrodynamics. While a number of simplifications were adopted (e.g. Sobolev approximation), this Monte Carlobased technique can be generalized to multidimensional geometries, and is planned to be applied for future studies of inhomogeneous outflows.

Thus, continuing the studies by Oskinova et al. (2007) on the physics of radiative transfer in resonance lines formed in clumped stellar winds and its applications for the empirical mass-loss rate diagnostics, significant work by different independent groups was done. Those studies largely confirmed and further developed the macroclumping (sometimes called porosity and vorosity) approach. Allowing for optically thick clumping in modelling resonance line in the UV spectra of massive stars resolves the previously reported discrepancy between mass-loss estimates based on $\rho$ and $\rho^{2}$ based diagnostics.

As a common result, Oskinova et al. (2007); Hamann et al. (2008), Šurlan et al. (2012, 2013) and Sundqvist et al. (2012b, 2014) agree that the mass-loss rates derived from optical and UV are a factor of a few $(2 \ldots 3)$ lower than predicted by the standard mass-loss recipe from Vink et al. (2000).

Interestingly, in a recent work by Shenar et al. (2015), where the X-ray, UV, and optical spectra of the O-type star $\delta$ Orionis were analysed consistently, it was shown that the empirically derived mass-loss rate $\dot{M} \approx 4 \times 10^{-7} M_{\odot} \mathrm{yr}^{-1}$ is only slightly below the predicted value $\left(\dot{M}_{\mathrm{Vink}} \approx 5 \times 10^{-7} M_{\odot} \mathrm{yr}^{-1}\right)$. This demonstrates that the analysis of stellar spectra is a complex task, and that it will become difficult (or even impossible) to provide a universal, exact and final number for the mass-loss reduction factor. 


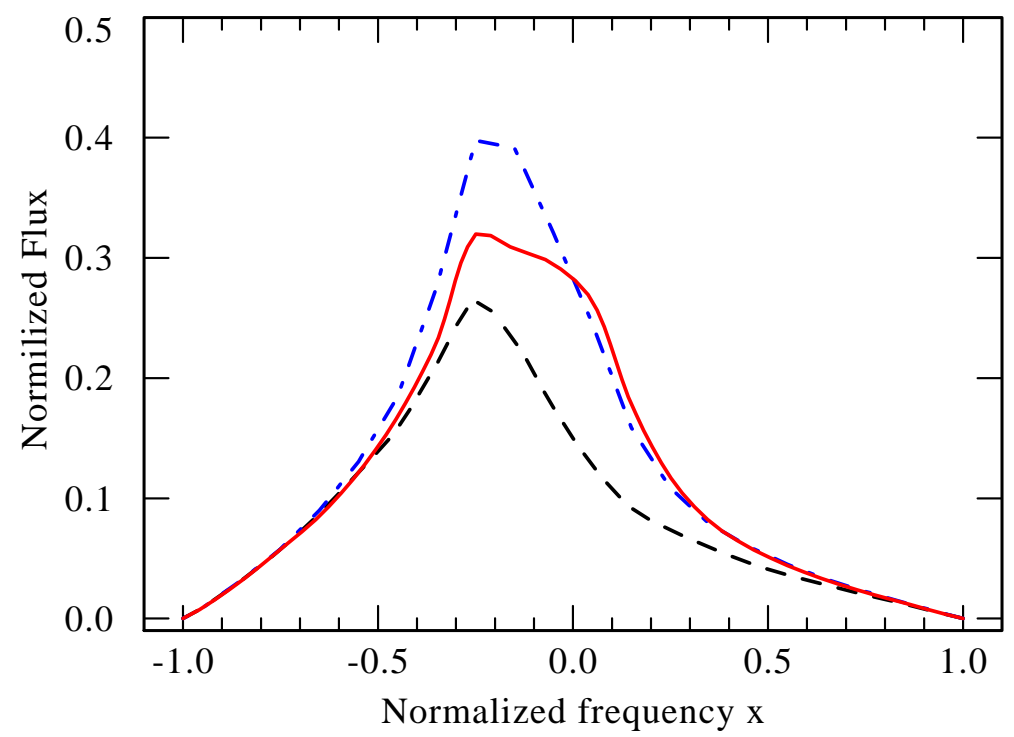

Fig. 6 Comparison of model X-ray line profiles. Three models are shown, the black dashed line is a smooth wind model, the blue dash-dotted line is a clumped wind model with spherical clumps, and the red solid line model with flat 'pancake' clumps. The computed line profiles are displayed as a function of dimensionless frequency $x$, measured relative to the line center and in Doppler units referring to the terminal wind velocity. The negative $x$ refers to blue frequencies. The stellar, wind, and model parameters are for the O-type star $\delta$ Ori (see Shenar et al., 2015, for details). The lines are normalized such that in the absence of any wind absorption the integrated line flux would be unity.

To conclude, macroclumping elevates a problem with severely reduced mass-loss rates from O-type stars. Abolishing the micro-clumping approximation from the modelling of UV and $\mathrm{X}$-ray spectra might allow to restore the standard picture, where the steady radiatively driven mass-loss from massive O-type stars could be sufficient to drive stellar evolution. However, it may be noted that already a factor of 2 to 3 lower mass-loss rates (compared to the massloss recipe by Vink et al. (2001) that is used in evolutionary models) as derived in many of the above investigations (see also the next sections) might be sufficient to modify present evolutionary predictions already at early phases, particularly regarding the evolution of the rotational velocity (Keszthelyi, 2015).

\subsection{X-ray spectroscopy with macroclumping}

Macroclumping may also elegantly explain the shapes of emission lines observed in X-ray spectra of O-type stars.

There is strong and direct observational evidence for wind clumping in O-type stars (e.g., Eversberg et al., 1998). It was suspected since long that stellar wind inhomogeneities affect the propagation of X-rays in stellar winds. The Einstein observatory discovered that OB stars are quite soft X-ray sources. Given that massive stars have dense stellar winds, it was apparently clear that the observed X-rays cannot originate from the stellar surface, since they would be completely absorbed in the overlaying dense stellar wind if it were smooth, and never reach the observer. Thus, the X-rays have to be generated in those wind regions, from where they can escape (Hillier et al., 1993). 


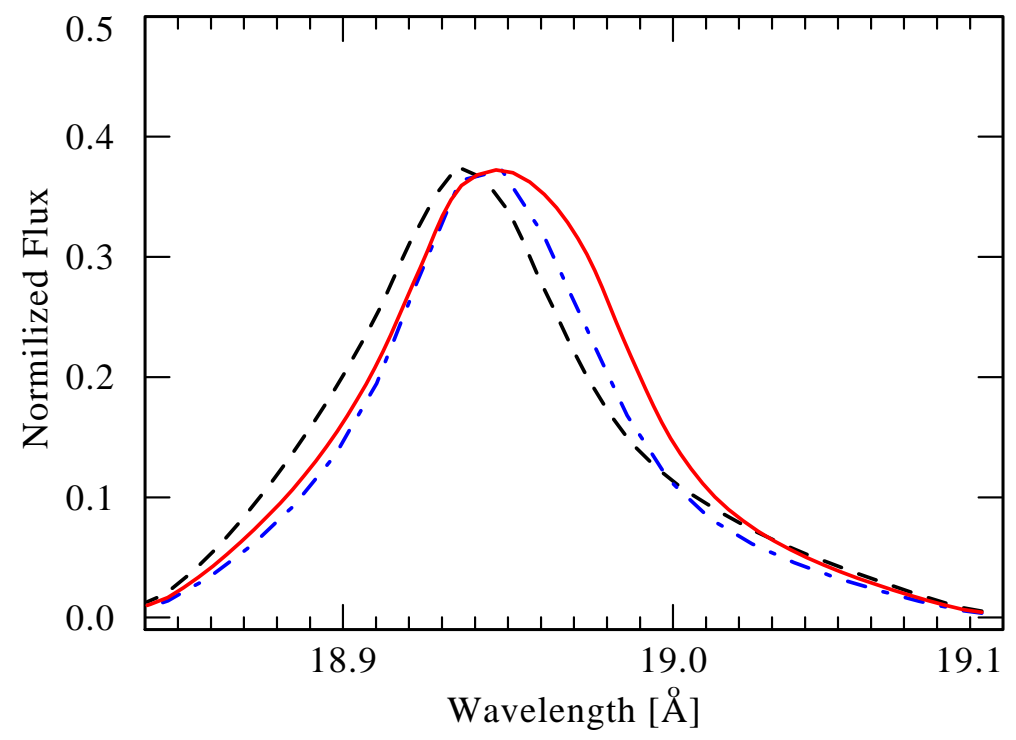

Fig. 7 The same as in Fig.6 but now the computed lines are displayed as a function of wavelength close to the O VIII $L \alpha$ line. The lines are re-normalized to the maximum as typically done in the analysis of the observed X-ray spectra, and convolved with the instrumental response of the Chandra HETGS MEG detector.

Stewart and Fabian (1981) used Einstein spectra of O-stars to study the transfer of Xrays through a uniform stellar wind as a means to determine stellar mass loss rates. They used a photoionization code to find the wind opacity. Using $\dot{M}$ as model parameter, they found from matching the model and the observed X-ray spectrum of $\zeta$ Pup that the X-ray based mass-loss rate from this star is a factor of a few lower than the mass-loss rates obtained from fitting the $\mathrm{H} \alpha$ line and the radio and IR excess. As a most plausible explanation for this discrepancy they suggest the neglected clumping in the stellar wind.

The first high-resolution spectra of O-type stars (Waldron and Cassinelli, 2001) showed nearly symmetric lines that can be well fitted by Gaussian functions, and do not show shapes predicted by Macfarlane et al. (1991) (see Fig.37). As a potential solution to this discrepancy Waldron and Cassinelli (2001) suggested inhomogeneous or non-symmetric winds. The idea that clumping may reduce the wind opacity and lead to more symmetric line profiles was also discussed by Owocki and Cohen (2001); Kramer et al. (2003). However, the first quantitative models for the shapes of emission lines emerging from a clumped stellar wind were computed by Feldmeier et al. (2003).

In Feldmeier et al. (2003) and Oskinova et al. (2004, 2006) a general formalism for Xray radiative transfer in a clumpy stellar wind was developed; while this formalism uses a phenomenological description of the clumpy wind, it attempts to take into account the basic picture emerging from 1-D LDI simulations as those discussed in Sect.2.1.2

The basic picture emerging from radiation hydrodynamic simulations of the non-linear evolution of instabilities in stellar winds is that of strong reverse shocks, which arise when a high-speed, rarefied flow impacts on slower material that has been compressed into dense shells (e.g., Owocki et al. 1988; Feldmeier et al. 1997a, see also Fig.2).

Building on this, the phenomenological 'broken-shell' model by Feldmeier et al. (2003) showed that the asymmetry of such broken shells lead to a characteristic shape of X-ray emission lines - the blueshifted part of X-ray line profiles remains flat-topped even af- 
ter severe wind attenuation, whereas the red part shows a steep decline (see Fig. 5,6 in Feldmeier et al., 2003).

The emission line models of Feldmeier et al. (2003) do not constrain clump optical depths - three cases were considered, optically thin clumps, optically thick clumps, and clumps with arbitrary optical depth (bridging between optically thin and thick cases). Feldmeier et al. (2003) considered a general structured stellar wind model - they did not use the outcome of a specific hydro-simulation, but rather concentrated on novel radiative transfer effects. On the other hand, Sundqvist et al. (2012b) applied a specific 1D LDI simulation (Feldmeier et al., 1997a) that was phased randomly among 3D patches, and computed parameterized emergent line profiles for a pre-specified value of $\tau_{0}$ by calculating the absorption directly from the actual density structure of the radiation-hydrodynamic model. They did not find significant porosity effects in their simulations.

Oskinova et al. (2004) used a 2.5-D stochastic wind model to study the propagation of $\mathrm{X}$-rays in structured winds. They considered a stochastic ensemble of clumps, allowing for a randomized mixture of clump optical depths at each radius and direction in the wind. It was shown that the clumped wind is much more transparent for the X-ray emission, even when the covering factor of the absorbing material is unity (i.e. when there are no gaps in the wind). They considered the limiting cases of optically thin and optically thick clumps, as well as the bridging case of arbitrary clump optical depths. A concept of effective opacity was introduced, and a thorough comparison with analytical models was done. It was shown that in the limiting case of optically thin clumps, the macroclumping formalism is identical to the microclumping or smooth wind formalism. Oskinova et al. (2006) compared the model X-ray line profiles with those observed in high-resolution X-ray spectra of $\mathrm{O}$ type stars, and concluded that wind clumping explains well the observed line shapes. They showed that macroclumping reduces the wavelength dependence of opacity, and in case of optically thick clumps, the opacity becomes grey. Importantly, Oskinova et al. (2006) demonstrated that macroclumping is a wavelength dependent effect - while for lines at higher energies the macroclumping is negligible, for lines at longer wavelength the macroclumping effects are important (see Fig. 8 and Fig. 9 in Oskinova et al., 2006).

Shaviv (1998) considered grey opacity in a stellar atmosphere where the photon mean free path does not exceed the scale of wind inhomogeneities. Shaviv (2000) coined the term "porous atmosphere" for massive stars to describe a multi-phase medium that allows more radiation to escape while exerting a weaker average force. Thus, in such atmosphere a considerably lower radiative acceleration results from the same total luminosity and, hence, can explain the apparent existence of super-Eddington stars (Shaviv, 2000).

Owocki et al. (2004) introduced a "porosity-length" formalism and applied it to a continuum dominated by electron scattering. In this approach, the porosity length turns out to be a photon mean free path for a medium consisting of optically thick clumps. This formalism was then used to obtain an associated scaling for the continuum-driven mass-loss rate from stars that formally exceed the Eddington limit, such as $\eta$ Car during its giant eruption in the 19th century.

Owocki and Cohen (2006) applied the porosity formalism to the case of wavelengths dependent K-shell opacity (i.e. to the same problem as was considered in Feldmeier et al., 2003). They considered spherical clumps, and developed an effective opacity law bridging the limits of optically thin and thick clumps; while the bridging law in Owocki and Cohen (2006) was chosen to allow for simple computations, it later turned out (Sundqvist et al., $2012 b$ ) that this form in fact represents that of an ensemble of clumps with local scales set by Markovian statistics. 


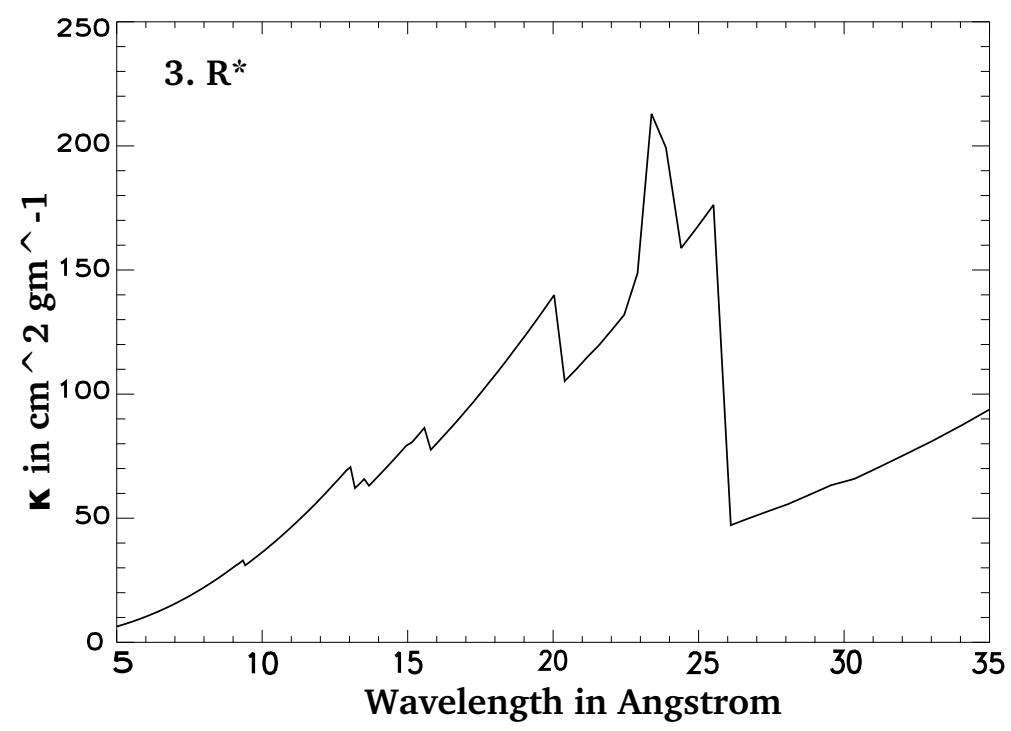

Fig. 8 Wavelength dependence of the mass absorption coefficient in the wind of the O-supergiant $\zeta$ Puppis at the distance in the wind $r \approx 3 R_{*}$. Adopted from Hervé et al. (2013).

As illustrated in Figures 6 and 7 the clump shape, i.e. the angular dependence of the clump cross-section, affects the shape of line profiles. Oskinova et al. (2006) showed that in case of spherically symmetric clumps, the line profiles are identical to those emerging from the smooth wind, albeit the same line shape would be achieved for larger $\dot{M}$ in case of a clumped wind as compared to the smooth wind. Thus, the fitting of line shapes alone cannot discriminate between the clumped and the smooth wind models. Note that this conclusion is in conflict with that reached by Leutenegger et al. (2013), who by fitting X-ray lines of $\zeta$ Pup showed that the profiles of smooth and isotropic porosity-models are only degenerate in the case of small porosity lengths, in which case the mass-loss rate correction from porosity is in any case marginal.

Macroclumping theory predicts that the wavelength dependence of stellar wind opacity for X-rays is reduced compared to smooth/microclumping models, i.e., winds are more grey for X-ray radiation (Oskinova et al., 2006). Actually, this is not only true for X-rays, but in all those cases where the clumps become optically thick to the specific radiation (Owocki et al., 2004).

Such a reduced wavelength dependency has been found in O-supergiant X-ray spectra by e.g. Kramer et al. (2003), who noticed that the wind opacity is nearly grey for X-rays in the wind of $\zeta$ Pup, while Rauw et al. (2015) found a rather modest wavelength dependence (at least longwards of $16 \AA$, see Sect. 3.10) of $\tau_{0}$ at $\approx 2 R_{*}$ in their analysis of $\lambda$ Cep 27 .

Waldron and Cassinelli (2007) presented an analysis of a broad collection of OB stellar line profile data to search for morphological trends. It was found in the majority of the OBstars that the emission lines are symmetric. But there is evidence for small, finite, blueward line shifts that also increase with stellar luminosity. This may be evidence for macroclumping playing an increasing role in OB-supergiant winds, compared to the stars of lower lu-

27 Cohen et al. (2014) find wavelength dependences of $\tau_{0}$ in their sample of $\mathrm{O}$ stars that are consistent with models without porosity; this is not surprising, since in the high frequency regime considered by Cohen et al. 2014 (below $\sim 15-20 \AA$ ) porosity is much less effective due to the lower opacity. 


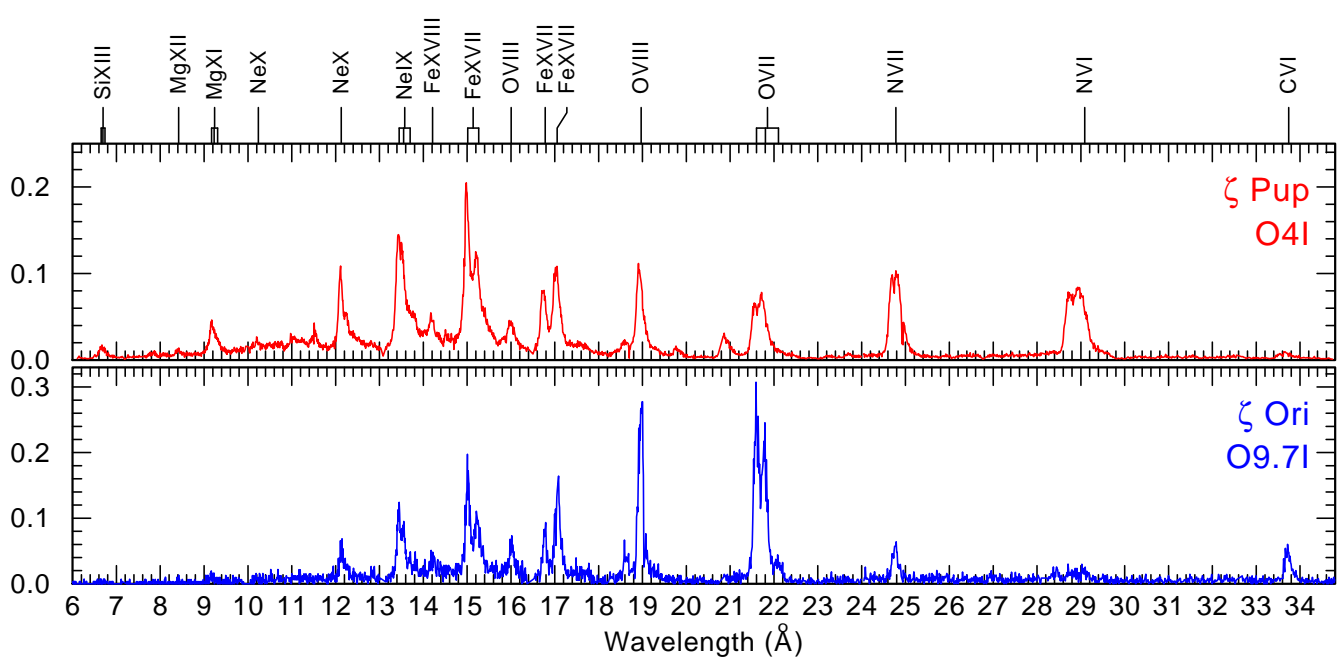

Fig. 9 An example of XMM-Newton RGS spectra of two prominent O-supergiant stars.

minosity classes. However, it may also simply be an effect of the increasing importance of absorption effects due to the higher supergiant mass-loss rates.

3.9 The size of clumps that attenuate X-rays

In the context of this review it is useful to provide estimates for the size of clumps that would attenuate X-rays, especially to make sure that such clumps indeed can be present in stellar winds in accordance with our knowledge of OB-supergiants. To obtain these estimates of clump sizes we follow the treatment discussed in Oskinova et al. (2011) and Oskinova et al. (2012).

Using Eq.50 the optical depth of an ideal spherical clump is

$$
\tau_{\text {clump }}(\lambda)=\rho_{\mathrm{w}} \kappa_{\lambda} D d_{\text {clump }} R_{*}=\tau_{0}(\lambda) \frac{d_{\text {clump }}}{f_{\mathrm{v}}} \cdot \frac{1}{\left(1-\frac{1}{r}\right)^{\beta} r^{2}},
$$

where $r=R / R_{*}$ and $d_{\text {clump }}$ is the characteristic geometrical size of the clump expressed in $R_{*}$. Note further that here the porosity length (mean free path between clumps), $h=$ $d_{\text {clump }} / f_{\mathrm{V}}$. The strong wavelength dependence of $\kappa_{\lambda}$ (see an example in Fig. 8) implies that the clump optical depth is wavelength dependent.

It is convenient to express $\tau_{0}(\lambda)$ (see Eq. 50) as

$$
\tau_{0}(\lambda) \approx 722 \frac{\dot{M}_{-6}}{v_{\infty} \mathscr{R}_{*}} \cdot \kappa_{\lambda},
$$

where $\dot{M}_{-6}$ is the mass-loss rate in units $10^{-6} M_{\odot} \mathrm{yr}^{-1}, v_{\infty}$ is the terminal velocity in $\left[\mathrm{km} \mathrm{s}^{-1}\right]$, and $\mathscr{R}_{*}=R_{*} / R_{\odot}$. If at some radius $r$, the clump size is larger than $d_{\text {clump }}^{\tau=1}$, such a clump is no longer optically thin for the $\mathrm{X}$-ray radiation at wavelengt $\lambda$. Assuming again a beta-velocity law, the size of a clump with optical depth $\tau=1$ at wavelength $\lambda$ is

$$
d_{\text {clump }}^{\tau=1}=\frac{f_{\mathrm{V}}}{\tau_{0}(\lambda)} \cdot\left(1-\frac{1}{r}\right)^{\beta} r^{2} .
$$


The microclumping approximation is valid only for significantly smaller clumps.

Let us estimate the geometrical size of a clump which has optical depth unity. We consider the O-type star $\zeta$ Pup and use its parameters from Bouret et al. (2012): $\dot{M}_{-6}=1.9$, $v_{\infty}=2300, R_{*}=18.8 R_{\odot}, \beta=0.9$, and a filling factor $f_{\mathrm{V}}=0.05$. Then $\tau_{0}(\lambda) \approx 0.03 \kappa_{\lambda}$.

Assuming a constant volume filling factor $f_{\mathrm{V}}=0.05$, and using values of $\kappa_{\lambda}$ at $3 R_{*}$ as shown in Fig. 8 the geometrical size of a clump optically thick for X-ray radiation at $\lambda=10 \AA$ and $r=3 R_{*}$ is $d_{\text {clump }} \approx 0.35 R_{*}$, while at $\lambda=24 \AA d_{\text {clump }} \approx 0.06 R_{*}$.

Though these estimates seem to be reasonable, the clump size and volume filling factor are actually not mutually exclusive, but related physically via the mean-free-path between clumps (i.e. via the porosity length $h=d_{\text {clump }} / f_{\text {vol }}$ ). Inserting the above numbers, one finds $h \approx 6.9 R_{*}$ at $10 \AA$ and $h \approx 1.2 R_{*}$ at $24 \AA$. This thus means that for clumps to become optically thick at $10 \AA$, they have to be individually separated by a (physical) mean free path $\sim 7 R_{*}$ (which is even much larger than the $3 R_{*}$ at which the computation were made). Thus, the clumps considered above cannot become optically thick at $10 \AA$, and their actual sizes will be smaller than calculated from the condition of unity optical depth.

$\mathrm{SgXBs}$ are quite hard X-ray sources, e.g. the maximum of the spectral energy distribution in Vela $X-1$ is at $\lambda \approx 4 \AA$, (e.g., Doroshenko et al., 2011). As shown in Oskinova et al. (2012), at a distance of $2 R_{*}$ from the stellar core, only geometrically large clumps with diameters larger than $\sim 0.5 R_{*}$ are optically thick for the ionizing radiation below $10 \AA$.

The analysis of the X-ray emission from the hard state of Cyg X-1 (a binary system hosting a supergiant star with a blackhole companion) presented by Grinberg et al. (2015) showed that an optical depth of order unity at $3 \mathrm{keV}$ can only be achieved if $h \approx 60 R_{*}$ (adopting the same parameters as above and the approximate scaling $\kappa_{\lambda} \approx 30 E_{\mathrm{keV}}^{-2}$, with $E_{\mathrm{keV}}$ the energy in $\mathrm{keV}$, e.g., Owocki et al. 2013). This illustrates that clumps can hardly be optically thick at those energies, similarly to what was concluded by Oskinova et al. (2012).

Moreover, and as also demonstrated above, due to the strong wavelength-dependence of the opacity, effects of macroclumping might indeed start to become relevant at longer wavelengths, and in particular when approaching the EUV part of the spectrum.

\subsection{X-ray spectroscopy neglecting macroclumping}

There are other approaches that allow to fit observed X-ray line profiles. In this section we briefly review these works.

As was pointed out in Oskinova et al. (2006), X-ray lines in O-star spectra are differently affected by clumping. Importantly, the best X-ray lines to probe the stellar wind structure are at the wavelengths where the wind opacity achieves the largest value. This is because the same wind clump would be optically thin at shorter wavelength and optically thick at longer ones, see Eq.52.

Due to its large effective area and softer response, the XMM-Newton RGS spectrograph is excellently suited to probe such lines. Figure 9 shows an example of X-ray spectra of two O-type stars. The wavelength dependence of the mass-absorption coefficient in the wind of $\zeta$ Pup is illustrated in Figure 8 As can be easily seen from a comparison of these two figures, the strongest lines at the wavelength where the cool wind opacity is largest and hence the lines that are best suited to probe wind clumping are O VIII $\lambda 18.97 \AA$, O VII $\lambda 21.6 \AA$, N VII $\lambda 24.78 \AA$ and N VI $\lambda 28.78 \AA$.

Leutenegger et al. (2013) fitted X-ray emission line profiles from high resolution $X M M$ Newton and Chandra grating spectra of the early $\mathrm{O}$ supergiant $\zeta$ Pup, to evaluate the relative importance of porosity and mass-loss rate in affecting the line shapes. Their method relies 
on fitting the Owocki and Cohen (2001) model mentioned above, with extensions to account for porosity and macroclumping according to Sundqvist et al. (2012b) and resonance line scattering according to Leutenegger et al. (2007). The model is specified by a rather small number of free parameters and implemented as local models in the global fitting software $X S P E C$, and the match to observed lines is then evaluated by a standard goodness of fit.

The conclusions are based on the formal $68.3 \%$ confidence limits obtained during the fitting procedure. Leutenegger et al. (2013) excluded from their analysis the diagnostic lines most sensitive to wind clumping, since they were aiming to primarily investigate the reliability of deriving mass-loss rates from (relatively high frequency) lines. Only lines blueward of $\lambda 20.91 \AA$, i.e. probing relatively low wind opacities, were thus considered. As already discussed in the previous paragraph, this might be a reason why only modest or no porosity effects were noticed in their analysis.

Leutenegger et al. (2013) demonstrated that neglecting macroclumping may lead to about $40 \%$ underestimate of mass-loss rates (see their section 4.3) for a porosity length $h \sim R_{*}$ when porosity effects are important, which, as outlined above, is a question of wavelength, mass-loss rate, velocity law, etc. Interestingly, such porosity lengths were recently derived from the analysis of X-ray variability in Cyg X-1 (Grinberg et al., 2015).

Indeed, the wavelength dependence of wind opacity implies that lines at shorter wavelengths $(<20 \AA)$ are less affected by wind absorption/clumping than the lines at longer wavelength. Thus, these lines are well suited to study the dynamics, emission measure, and spatial distribution of the hot plasma.

Consistent analyses of spectral lines, including important wind diagnostic lines of $\mathrm{O}$ and $\mathrm{N}$, in the X-ray spectra of $\zeta$ Pup (O4 Ief) and its spectroscopic twin $\lambda$ Cep (O6 Ief) were performed by Hervé et al. (2013) and Rauw et al. (2015). Both these works neglected the influence of porosity on the analysed X-ray emission lines (based on previous results see above - for lines at not too long wavelengths), and yet were able to achieve excellent fits to the observed X-ray spectra for realistic stellar wind parameters. This was achieved by introducing a new free parameter - the radial distribution of the hot plasma filling factor.

It is important to note that Cohen et al. (e.g., 2010b) tested models with varying filling factors by fitting them to observed X-ray emission line profiles. They concluded that including a radial dependence of the filling factor does not improve the line fits. Hence, they suggested that a radial dependence of the filling factor can be neglected.

In their analysis Rauw et al. (2015) employ the cool wind opacity as computed using the non-LTE stellar atmosphere model CMFGEN. This numeric stellar atmosphere provides theoretical predictions for the parameter $\tau_{0}(\lambda, r)$ (see Eq. 50). The same parameter can be empirically estimated by fitting the Owocki and Cohen (2001) model to the observed line profiles. Then, the predictions from the NLTE wind model and the empirically estimated values can be compared.

Studying the X-ray emission line profiles, Rauw et al. (2015) demonstrated that the Owocki and Cohen (2001) models cannot easily explain the morphology of the NVII Ly $\alpha$ line, though some other used lines (NeX, FeXVII and OVIII, with $\lambda<20 \AA$ ) were matched almost perfectly. Notably, the wind opacity derived using this formalism is smallest at the wavelength of the N VII $\lambda 24.78 \AA$ line (see Table 4 in Rauw et al. (2015)), in contradiction to the expectations from the NLTE stellar wind models.

To achieve satisfactory fits for all lines of the X-ray spectrum of $\lambda$ Cep, Rauw et al. (2015) introduced a model, where the stellar wind is divided into a grid of concentric shells, each with own temperature and emission measure of X-ray emitting gas. The temperature and the emission measure filling factor is then varied until the model is a good agreement with observations. As previously, the cool wind opacity is provided by the NLTE atmo- 
sphere with specified mass-loss rate, velocity field and other stellar parameters. The good correspondence between the model and the observations is achieved for mass-loss rates in agreement with the one obtained from fitting the UV lines with the models that account for macroclumping (see Figs.45) as derived by Šurlan et al. (2013); Sundqvist et al. (2014). Remember, that macroclumping effects needed to be included in the UV-analysis (optically thick clumps with respect to UV-lines), but were excluded from the X-ray analysis. The best fit model constrains the location of the X-ray plasma in $\lambda$ Cep between $1.1 R_{*}$ and $2.5 R_{*}$, with a non-monotonic radial distribution of the filling factor.

Despite the fact that $\lambda$ Cep is a spectroscopic twin of $\zeta$ Pup in the optical and in X-rays, there are very different conclusions about the filling factor distribution and the location of hot gas in these stars. While the results from Hervé et al. (2013) and Leutenegger et al. (2013) allow the presence of hot plasma at very large distances from the star, the results of Rauw et al. (2015) require a finite radius for the X-ray emitting gas. In this respect, this is similar to the approach of Oskinova et al. (2006).

It seems that numeric hydrodynamic simulations could accommodate non-monotonic filling factors, but it is difficult to reconcile the hydrodynamic models with such a sharp cut-off of X-ray emitting gas at $2.5 R_{*}$ in $\lambda$ Cep (see Krtička et al. 2009).

Waldron and Cassinelli (2007) studied the spatial distribution of hot gas in OB stars from an analysis of their X-ray spectra. They found that the highest temperatures occur near the star and steadily decrease outward. This trend seemed to be most pronounced in OB supergiants. For the lower density wind stars, both high and low X-ray source temperatures may exist near the star. They called this intriguing temperature distribution the "near-star high-ion problem" for OB stars. By invoking the traditional OB stellar massloss rates, Waldron and Cassinelli (2007) found a good correlation between the spatial onset of X-ray radiation and X-ray continuum optical depth unity radii. On the other hand Leutenegger et al. (2013) used the observed flux in the far wings of various X-ray lines, and from this inferred hot gas at high temperatures far away from the star directly from observations of $\zeta$ Pup.

Observational constraints on the radial stratification of the clumping filling factor of the cool wind material were provided in Puls et al. (2006). From the study of own and archival data for $\mathrm{H} \alpha$, IR, mm and radio fluxes, and using approximate methods, calibrated to more sophisticated models, it was shown that the minimum clumping factor (except for the lowermost unclumped wind with $r<1.1 R_{*}$ ) is found in the outermost, radio-emitting region 28 . Thus, the radio mass-loss rates would be the lowest ones, compared to those derived from $\mathrm{H} \alpha$ and the IR, when analysed by means of unclumped models. These radio rates (assuming $D=1$ in the outermost wind) agree well with those predicted by Vink et al. (2000), but are only upper limits, since the absolute value of $D$ could not be constrained from this pure micro-clumping investigation, and might be larger than unity. For denser winds, it turned out that the inner wind region (from $r>1.1 R_{*}$ ) is more strongly clumped than the outermost one (with a normalized clumping factor of $\approx 4$ ), whereas thinner winds have similar clumping properties in the inner and outer regions.

An interesting application of X-ray spectroscopy of stellar winds is its usefulness to constrain the absorbing column density of cool wind material. Indeed, the stellar mass-loss rate has a direct influence on the shape of the X-ray emission lines (see Fig. 10 adopted from Oskinova et al. (2006)). In this respect, Oskinova et al. (2006); Hervé et al. (2013); Shenar et al. (2015); Rauw et al. (2015) demonstrated that X-ray spectroscopy is a very use-

\footnotetext{
28 The clumping factor used in this (and other) work corresponds to the over-density $D=f_{V}^{-1}$ introduced in Sect. 2.1
} 


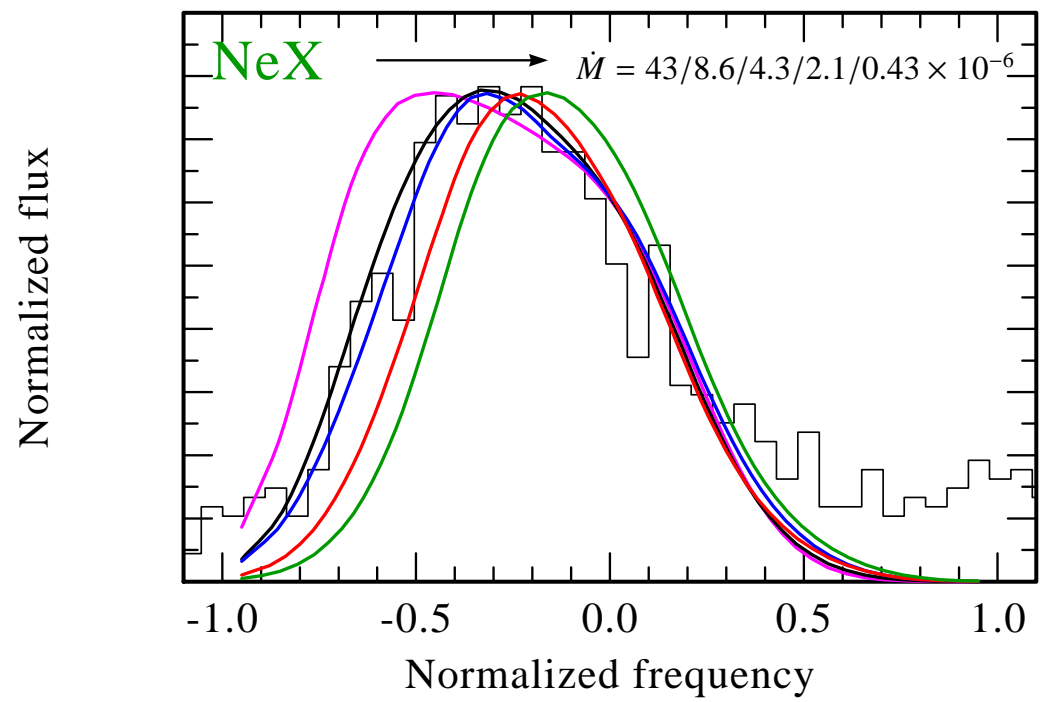

Fig. 10 Observed (histogram, Chandra X-ray telescope) and modelled Ne $\mathrm{X}$ line in $\zeta$ Pup. From left to right, model line profiles are calculated assuming $\dot{M}=43,8.6,4.3,2.1,0.43 \times 10^{-6} M_{\odot} \mathrm{yr}^{-1}$. All synthetic lines have been normalized to the observed maximum flux. Adopted from Oskinova et al. (2006)

ful tool to constrain stellar mass-loss rates, especially when used in combination with NLTE modelling of UV/optical spectra.

A different approach was used by Cohen et al. (2014). Based on their earlier work, they suggested to directly measure mass-loss rates from the observed X-ray line profiles, which should result in an unbiased value from all lines with negligible porosity (see above). By this method, they found mass-loss rates for O-stars which are, interestingly, also a factor of 2 to 3 (or even more) lower than the corresponding rates from Vink et al. (2001), in accordance with most results from macro-clumped UV-diagnostics (see above), and also consistent with optical $\mathrm{H} \alpha$ when clumping is accounted for (see Cohen et al. 2014). Future work shall demonstrate how useful and robust this method is.

In summary, this brief review does not cover all available models and approaches to model $\mathrm{X}$-ray emission lines and spectra. Mainly, we concentrated here on the influence of clumping/porosity on UV and X-ray line profiles. Some of the models we considered do not favour the presence of optically thick clumping for X-rays in stellar winds. Others provide a satisfactory description of observed spectra allowing variable filling factors of hot gas. In another approach the optical depth of clumps in stellar wind is not constrained, and clumps can be optically thick for X-rays, depending on wavelength and mass-loss rate. Future observations and modelling work will either allow to discriminate between the importance of non-monotonic X-ray filling factors and clumping in O-star winds, or provide evidence for the co-existence of both effects.

\section{Stellar wind parameters from X-ray observations of classical SgXBs and SFXTs}

The class of high mass X-ray binaries (HMXBs) with supergiant companions, is composed by two sub-classes: the classical systems or $\mathrm{SgXBs}$, like Vela X-1 already mentioned earlier 
Table 2 Wind-accreting Supergiant X-ray Binary systems with neutron stars: (A) SgXBs (B) SFXTs.

\begin{tabular}{|c|c|c|c|c|c|c|}
\hline Name & Companion & $\begin{array}{l}\text { Distance } \\
\mathrm{kpc}\end{array}$ & $\begin{array}{l}\text { Orbital } \\
\text { Period (d) }\end{array}$ & $\begin{array}{l}\text { Spin } \\
\text { Period (s) }\end{array}$ & $\begin{array}{l}\text { Super-Orbital } \\
\text { Period (d) }\end{array}$ & $\begin{array}{l}\text { Current } \\
\text { Class. }\end{array}$ \\
\hline $1 \mathrm{~A} 0114+650$ & B0.5I ${ }^{1}$ & $7.2^{2}$ & $11.59^{1}$ & $9475^{3}$ & $30.7^{4}$ & $\mathrm{~A}$ \\
\hline Vela X-1 & B0.5Ib & $1.7-2.1^{5}$ & $8.964^{6}$ & $283^{6}$ & - & A \\
\hline 1E 1145.1-6141 & B2Iae ${ }^{7}$ & $8.2^{7}$ & $14.365^{8}$ & $297^{9}$ & - & A \\
\hline GX 301-2 & $\mathrm{B} 1 \mathrm{Ia}+{ }^{10}$ & $3-4^{10}$ & $41.492^{11}$ & $675700^{10}$ & - & A \\
\hline $4 U$ 1538-522 & $\mathrm{B} 0 \mathrm{I}^{12}$ & $5.5^{12}$ & $3.728^{13}$ & $528-530^{14}$ & - & A \\
\hline IGR J16318-4848 & $\operatorname{sgB}[e]^{15}$ & $0.9-6.2^{15}$ & - & - & - & A \\
\hline IGR J16320-4751 & O8I ${ }^{16}$ & $3.5^{16}$ & $8.986^{17}$ & $1309^{18}$ & - & A \\
\hline IGR J16393-4641 & OB $?^{19}$ & $>10 ?^{19}$ & $4.24^{20}$ & $912.0^{21}$ & - & A \\
\hline IGR J16465-4507 & $09.5 \mathrm{Ia}^{22}$ & $9.5^{23}$ & $30.243^{24,25}$ & $228^{26}$ & - & A \\
\hline IGR J16493-4348 & B0.5 $\mathrm{Ib}^{27}$ & $>6^{27}$ & $6.782^{28}$ & $1093^{28}$ & $6.8^{29}$ & A \\
\hline OAO $1657-415$ & Ofpe/WN9 $9^{30}$ & $4-8^{30}$ & $10.448^{30}$ & $38.2^{31}$ & - & A \\
\hline $4 \mathrm{U} 1700-37$ & O6.5 Iaf+ 32 & $1.9^{33}$ & $3.412^{34}$ & - & - & A \\
\hline EXO 1722-363 & $\mathrm{B} 0-\mathrm{B} 1 \mathrm{Ia}^{35}$ & $6-10.5^{36}$ & $9.742^{37}$ & $413.89^{38}$ & - & A \\
\hline IGR J18027-2016 & $\mathrm{B} 1 \mathrm{Ib}^{39}$ & $12.4^{39}$ & $4.469^{39}$ & $139.612^{40}$ & 一 & A \\
\hline XTE J1855-026 & B0 Iaep ${ }^{41}$ & - & $6.0724^{42}$ & $360.7^{43}$ & - & A \\
\hline 4U 1907+097 & O8Ia,O9Ia ${ }^{44}$ & $5^{44}$ & $8.3753^{44}$ & $437-441^{45}$ & - & A \\
\hline 4U 1909+07 & B1-B3 (I) ${ }^{46}$ & $4.85 \pm 0.50^{46}$ & $4.4^{47}$ & $605^{46}$ & $15.180^{39}$ & A \\
\hline IGR J19140+0951 & $\mathrm{B} 0.5 \mathrm{Ia} / \mathrm{d}^{47}$ & $2-5^{47}$ & $13.55^{48}$ & - & - & A \\
\hline IGR J16207-5129 & $\mathrm{BOI}^{42}$ & $6^{49}$ & $9.726^{50}$ & - & - & A \\
\hline IGR J16418-4532 & $\overline{\mathrm{OB} \mathrm{Sg}}$ & $13^{51}$ & $3.753^{52}$ & $1212^{53}$ & $14.6842^{54,55}$ & $A$ or $B^{56}$ \\
\hline XTE J1739-302 & O8Iab(f) $)^{57,58}$ & $2.7^{58}$ & $\overline{51.47^{59}}$ & - & - & $\mathrm{B}$ \\
\hline IGR J17544-2619 & $\mathrm{O} 9 \mathrm{Ib}^{60}$ & $3.0 \pm 0.2^{60}$ & $4.926^{61}$ & $71.49 / 11.6^{62}$ & - & B \\
\hline SAX J1818.6-1703 & $\sim \mathrm{B} 0 \mathrm{I}^{63,64}$ & $2,2.1^{65,66}$ & $30.0^{67,68}$ & - & - & B \\
\hline IGR J16479-4514 & O8.5I, O9.5Iab ${ }^{58,42}$ & $4.9,2.8^{58,42}$ & $3.3194^{68,69}$ & - & $11.880^{54,55}$ & B \\
\hline IGR J18483-0311 & B0.5Ia 70 & $3^{70}$ & $18.55^{71}$ & $21.0526^{72}$ & - & B \\
\hline IGR J18450-0435 & $09.5 \mathrm{I}^{73}$ & $3.6^{73}$ & $5.7195^{74}$ & - & - & B \\
\hline IGR J18410-0535 & $\mathrm{B} 1 \mathrm{Ib}^{14}$ & $3.2^{75}$ & - & - & - & B \\
\hline IGR J08408-4503 & $08.5 \mathrm{Ib}^{76}$ & $2.7^{77}$ & $9.5436^{78}$ & - & $285 \pm 10^{78}$ & B \\
\hline IGR J11215-5952 & $\mathrm{B} 0.5 \mathrm{Ia}^{79,80}$ & $6.2,8^{81,79}$ & $164.6^{82,83}$ & $186.78^{84}$ & - & B \\
\hline IGR J16328-4726 & O8Iafpe ${ }^{85,86,87}$ & $3-10^{87}$ & $10.068^{88,89}$ & - & 一 & B \\
\hline IGR J18462-0223 & & & & & & $\mathrm{B}^{90}$ \\
\hline
\end{tabular}

(1) Crampton et al. 1985; (2) Reig et al. 1996; (3) Wang 2011; (4) Farrell et al. 2006; (5) Nagase et al. 1986; (6) Quaintrell et al. 2003; (7) Densham and Charles 1982; (8) Rav and Chakrabarty 2002; (9) White et al. 1980; (10) Kaper et al. 2006; (11) Koh et al. 1997; (12) Revnolds et al. 1992; (13) Clark et al. 1994; (14) Clark 2000; (15) Filliatre and Chaty 2004; (16) Rahoui et al. 2008; (17) Corbet et al. 2005; (18) Lutovinov et al. 2005; (19) Bodaghee et al. 2012; (20) Pearlman et al. 2011; (21) Bodaghee et al. 2006; (22) Coe et al. 1996; (23) Clark et al. 2010; (24) La Parola et al. 2010; (25) Walter et al. 2006; (26) Nespoli et al.|2010; (27) Pearlman et al.|2013; (28) Corbet et al.|2010b; (29) Mason et al. 2012; (30) White and Pravdo 1979; (31) Jones et al. 1973; (32) Ankav et al.|2001; (33) Corbet et al. 2010; (34) Mason et al. 2009; (35) Manousakis and Walter 2011; (36) Masetti et al. 2008; (37) Torrejón et al. 2010b; (38) Mason et al. 2011; (39) Negueruela et al. 2008; (40) Corbet et al. 1999; (41) Corbet and Mukai 2002; (42) Nespoli et al. 2008; (43) in't Zand et al. 1998; (44) Cox et al. 2005; (45) Fritz et al. 2006; (46) Martínez-Núñez et al. 2015; (47) Hannikainen et al. 2007; (48) Corbet et al. 2004; (49) Negueruela et al. 2007. (50) This period has been reported by Jain et al. 2011 but never confirmed; (51) Chaty et al. 2008; (52) Corbet et al. 2006; (53) Sidoli et al. 2012; (54) Corbet and Krimm 2013; (55) Drave et al.2013a; (56) This source has been considered as SgXB by Romano et al. 2014b Drave et al. 2013b and as SFXT by Paizis and Sidoli 2014 since it displays hard Xray properties, in term of cumulative luminosity ditribution of the flares, compatible with other SFXTs; (57) Negueruela et al. 2006b; (58) Rahoui et al. 2008; (59) Drave et al. 2010; (60) Giménez-García et al. 2016; (61) Clark et al. 2009; (62) A possible spin period of $71.49 \pm 0.02$ was reported by Drave et al. 2012 but this result was not confirmed and questioned by (Drave et al., 2014); Romano et al. 2015 reported instead a marginal evidence for a periodicity at $\sim 11.6 \mathrm{~s}$ which could be associated with the neutron star spin period; (63) Negueruela and Smith 2006 (64) Torreión et al. 2010a; (65) Negueruela et al. 2008; (66) Zurita Heras and Chaty 2009; (67) Bird et al. 2009; (68) Jain et al. 2009; (69) Romano et al. 2009b; (70) Rahoui and Chaty 2008; (71) Levine and Corbet 2006; (72) Sguera et al. 2007 but note that their results were criticized by (Ducci et al., 2013); (73) Coe et al. 1996; (74) Goossens et al. 2013; (75) Walter et al. 2006; (76) Barba et al. 2006; (77) Leyder et al. 2007; (78) Gamen et al. 2015; (79) Negueruela et al. 2005; (80) Lorenzo et al.|2010; (81) Masetti et al.|2006; (82) Sidoli et al. 2006; (83) Sidoli et al. 2007; (84) Swank et al. 2007; (85) Hanson et al. 1996; (86) Coleiro et al. 2013; (87) Fiocchi et al. 2010; (88) Corbet et al. 2010a; (89) Fiocchi et al. 2013; (90) Sguera et al. 2015 
in this review, and the Supergiant Fast X-ray Transients (SFXTs). While these systems share a number of common properties, their variability behavior in the X-ray domain is significantly different. As we discuss in the following sections, observations of these systems can provide us with information on the physical properties of massive star winds. For instance, recently a comparative analysis of the optical companion winds between the SgXBs Vela X-1 and the SFXT IGR J17544-2619, pointed to a substantial difference in their terminal velocities, being $\sim 1500 \mathrm{~km} \mathrm{~s}^{-1}$ in the case of IGR J17544-2619 and $\sim 700 \mathrm{~km} \mathrm{~s}^{-1}$ in Vela X-1 (Giménez-García et al., 2016).

We summarise shortly below the present knowledge about classical SgXBs and SFXTs. We refer the reader to Walter et al. (2015) for a recent and more extended review of these systems.

Supergiant X-ray Binaries ( $\mathrm{SgXBs}$ ) are among the first detected galactic X-ray sources e.g., Cyg X-1 or Vela X-1. They consist of OB supergiant mass donors and a compact object accreting from the strong stellar wind. Table 2 lists all known $\mathrm{SgXBs}$ harbouring as a compact object a neutron star.

The classical $\mathrm{SgXB}$ s are persistent systems in X-rays, displaying a moderate X-ray luminosity achieving $10^{36}-10^{37} \mathrm{erg} \mathrm{s}^{-1}$. The X-ray emission from $\mathrm{SgXBs}$ is usually characterised by a typical short-term variability, comprising flares and "off-states" that can reach a dynamical range of $\sim 10-100$ over time-scales of few to hundred of seconds. As briefly anticipated in Sect. 1 this variability is ascribed to the presence of inhomogeneities in the wind material that is accreted onto the neutron star or to switches between different accretion regimes. We discuss these aspects in more details in Sect.4.1 As an example of the SgXBs $\mathrm{X}$-ray variability, we show in the left panel of Fig. 11 a typical lightcurve from Vela X-1 as observed in the soft X-ray domain. The corresponding X-ray spectrum is reported in the left panel of Fig. 12 (together with a second example of an X-ray spectrum for the classical SgXB GX 301-2).

The spectrum of Vela X-1, as for other SgXBs, displays a large absorption column density $\left(N_{\mathrm{H}} \gg 10^{22} \mathrm{~cm}^{-2}\right)$ that is usually ascribed to the dense wind material from the supergiant companion filling the neutron star surroundings. Monitoring observations of these sources at different orbital phases can thus be used to measure changes in the absorption column density and probe the massive star wind on large scales (see Sect.4.4.1). These observations are also particularly useful to reveal the presence of long-lived dense structures around the compact objects that are usually associated to the disruption of the stellar wind by the gravitational field and X-ray radiation from the compact object (see Sect.4.2).

The relatively complex continuum emission can be described by using one or more power-law components, typically interpreted in terms of Comptonization of the thermal photons from the neutron star surface in its accretion column. Several emission lines with energies comprised in the range $2-8 \mathrm{keV}$ are also usually detected in the spectra of Vela X-1 and other classical SgXBs. These are produced as a consequence of the fluorescent emission of X-rays from the neutron star on the surrounding wind material. The most prominent line is the $\mathrm{Fe} \mathrm{K} \alpha$ from neutral iron and/or low-ionization stages, which has a centroid energy of $6.4 \mathrm{keV}$. Fluorescence from Fe ions at higher ionization states can give rise to emission lines in the range 6.7-6.9 keV. The weaker line detected usually around $7.1 \mathrm{keV}$ corresponds to the $\mathrm{Fe} \mathrm{K} \beta$ line. In sources with particularly high absorption column densities $\left(N_{\mathrm{H}} \gg\right.$ $10^{23} \mathrm{~cm}^{-2}$ ) other fluorescent lines can also be detected (e.g., the Ni $\mathrm{K} \alpha, \mathrm{Ca} \mathrm{K} \alpha$, Ar K $\alpha$, and $\mathrm{S} \mathrm{K} \alpha$; see right panel of Fig. 12]. We exploit the usability of these fluorescence features as probes of the massive star wind in Sect.4.3.

High resolution X-ray spectra of classical $\mathrm{SgXBs}$ carried out with the gratings on-board $X M M-N e w t o n$ and Chandra revealed also the presence of other much less prominent emis- 
Vela X-1 (XMM-Newton)

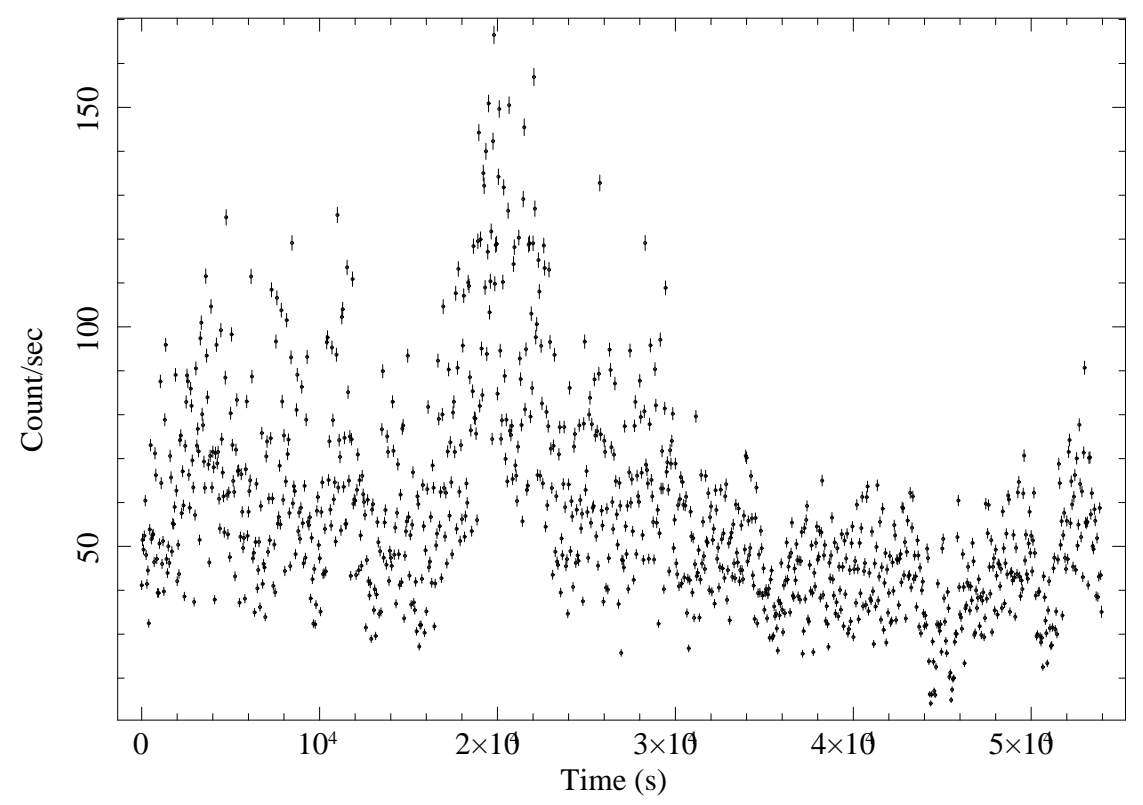

IGR J17544-2619 (XMM-Newton)

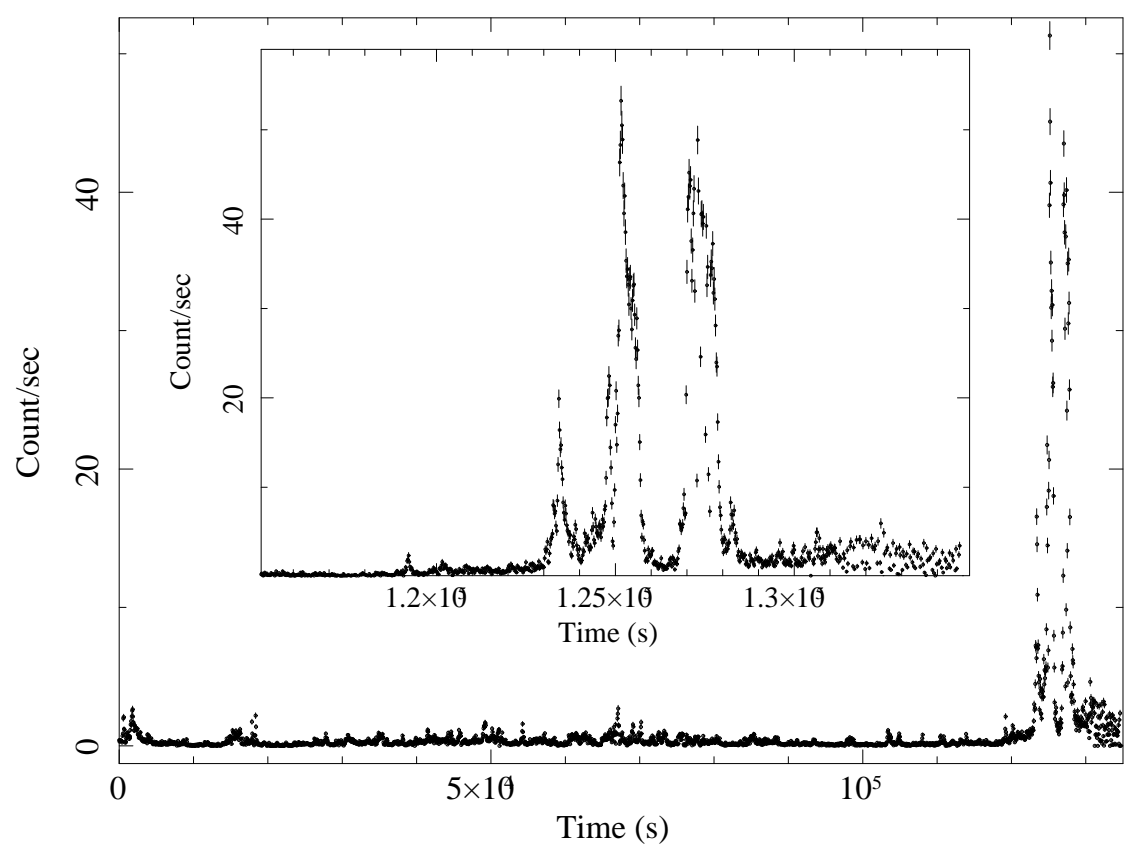

Fig. 11 Top: An example of a classical SgXB lightcurve as observed by XMM-Newton (0.5-10 keV). In this case the source is Vela X-1 (see Table 2). The observation (ID. 0111030101) was carried out on November 2, 2000 for about $60 \mathrm{ks}$. The flaring behaviour is evident, with X-ray flux variations by a factor of $\sim 3-10$ during the entire observation (see Martínez-Núñez et al., 2014, for more details on the XMM-Newton data analysis and results for this source). This variability can be reasonably well explained by assuming that the accretion on the neutron star hosted in this system is taking place from a highly structured and clumpy stellar wind. Bottom: an example of an SFXT lightcurve as observed by XMM-Newton (0.5-10 keV). In this case the source is the SFXT prototype IGR J17544-2619 and the lightcurve is obtained from the longest continuous observational campaign performed in X-rays on one of these sources (ID. 0744600101; (Bozzo et al., 2016)). The more extreme variability compared to the classical $\mathrm{SgXB}$ can be immediately seen by comparing this lightcurve with that on the top panel. A simple clumpy wind accretion model is not able to fully explain the SFXT behaviour in X-rays, and additional complications have to be taken into account (see Sect. 4.5. 
Vela X-1 (XMM-Newton)

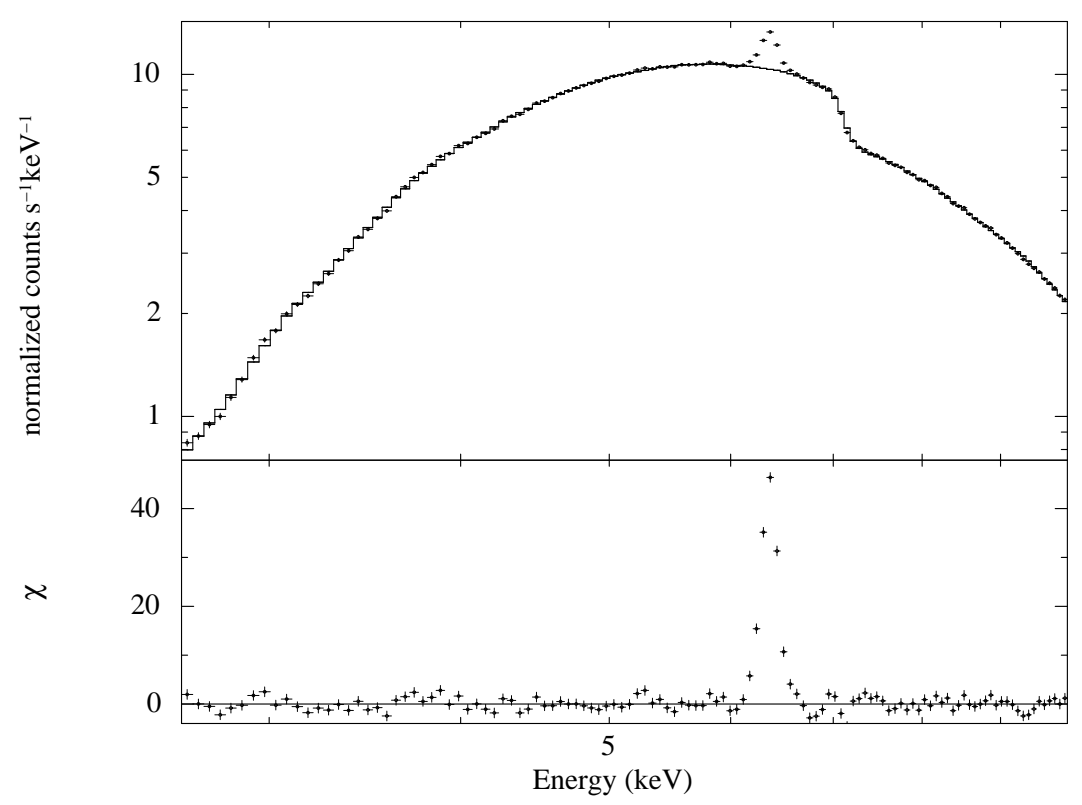

GX 301-2 (XMM-Newton)

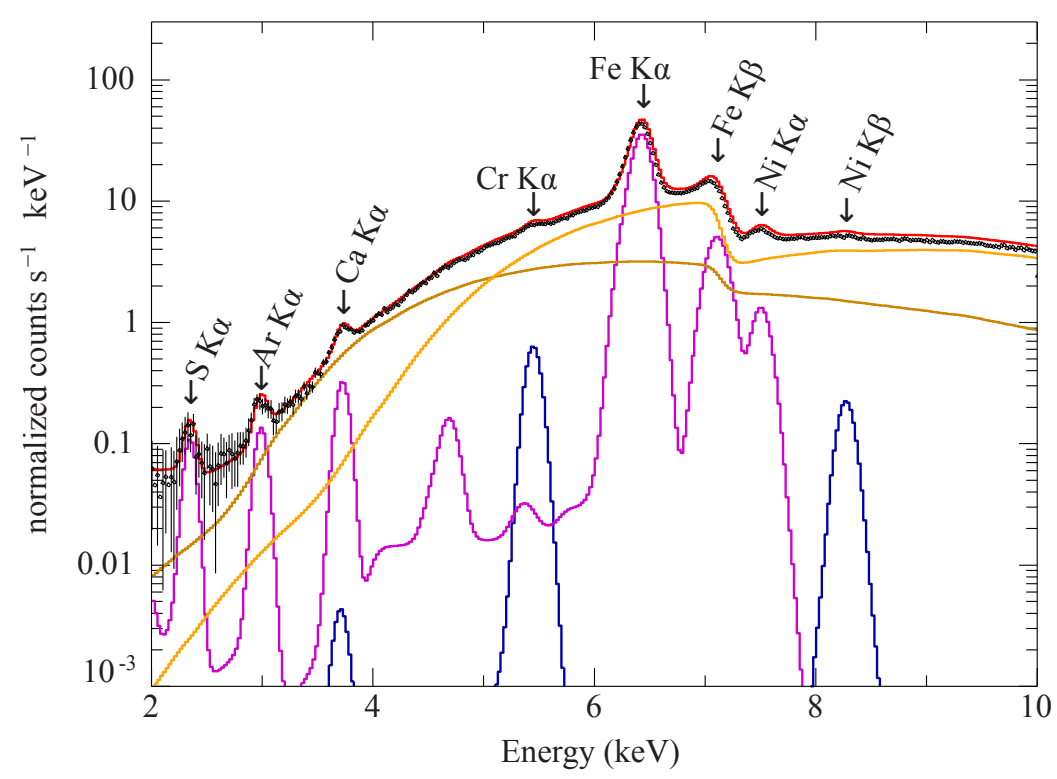

Fig. 12 Top: An example of an X-ray spectrum of Vela X-1. This corresponds to the average source spectrum extracted during the XMM-Newton observation ID. 0111030101 (see Fig. 111). The continuum of the spectrum is preliminarily described by the sum of two power-law components (one including a high energy cut-off) and a prominent iron $\mathrm{K} \alpha$ line line at $6.4 \mathrm{keV}$ (see Sect.4.3. The latter can be clearly seen in the residuals from the fit when it is not included in the spectral model (bottom panel). The average absorption column density measured from Vela X-1 during the XMM-Newton observation is $N_{\mathrm{H}} \sim 2 \times 10^{23} \mathrm{~cm}^{-2}$.

Bottom: Another example of an X-ray spectrum from a classical SgXB. In this case the source is GX 301-2, the $\mathrm{SgXB}$ characterized by one of the highest intrinsic absorption column density $\left(N_{\mathrm{H}} \sim 10^{24} \mathrm{~cm}^{-2}\right)$. The continuum emission is described by a partial covering plus an absorbed power-law model. A number of prominent emission lines are visible above the continuum. These are the $\mathrm{Fe} \mathrm{K} \alpha, \mathrm{Fe} \mathrm{K} \beta, \mathrm{Ni} \mathrm{K} \alpha, \mathrm{Ca} \mathrm{K} \alpha$, $\operatorname{Ar~K} \alpha$, and $\mathrm{S} \alpha$ fluorescence lines (see Fürst et al., 2011, for details about the data analysis and results). Reproduced with permission from Fürst et al. (2011). 
sion lines at energies of $\lesssim 3 \mathrm{keV}$ that are typically produced by radiative decays of highly ionized ions (e.g., S, Si, Mg, Ne; see Watanabe et al., 2006, and references therein). These features are particularly important to investigate the properties of the stellar winds, as the measured Doppler shifts in their centroid energies allow us to derive accurate estimates of the relative velocity between the neutron star and the surrounding material. This is discussed in Sect.4.2

A peculiar sub-class of SgXBs is that of the SFXTs (Sguera et al., 2005; Negueruela et al. 2006a; Sguera et al., 2006). At present, about $10 \mathrm{SgXBs}$ belong to this sub-class, with the prototype being IGR J17544-2619 (Sunyaev et al., 2003). An overview of the confirmed SFXT sources is reported in Table 2 Although the SFXTs host neutron stars accreting from similar supergiant stars as those in classical $\mathrm{SgXBs}$, they display a much more pronounced variability in X-rays, with a typical dynamical range that can reach $10^{5}-10^{6}$, compared with a factor 10 in Vela X-1 during a giant flare. The SFXTs achieve an X-ray luminosity as high as the typical average value of classical $\mathrm{SgXBs}$ only sporadically during their peculiar short outbursts. These events last a few thousand of seconds at the most and reach $10^{37}$ $10^{38} \mathrm{erg} \mathrm{s}^{-1}$ (see, e.g. Rampy et al., 2009; Romano et al., 2015; Bozzo et al., 2011). We show an example of an SFXT lightcurve in the soft X-ray domain (0.5-10 keV) in the lower panel of Fig. 11, where a bright flare is suddenly detected after an extended period of low $\mathrm{X}$-ray activity.

The amount of time that the SFXTs spend in these bright flares is only a few \% (Paizis and Sidoli, 2014; Romano et al., 2014a). For most of the time they are detected at an intermediate emission state with a typical luminosity of $10^{33}-10^{34} \mathrm{erg} \mathrm{s}^{-1}$, during which less prominent flares are observed. The latter are reminiscent of the brightest outburst (i.e. they occur on the same time-scales), but their peak luminosity is $\lesssim 10^{35} \mathrm{erg} \mathrm{s}^{-1}$ (Sidoli et al., 2008; Bodaghee et al., 2011; Sidoli et al., 2010; Bozzo et al., 2010). A number of SFXTs were also observed in a quiescent state, with luminosities down to $10^{32} \mathrm{erg} \mathrm{s}^{-1}$ (González-Riestra et al., 2004; in't Zand, 2005; Leyder et al., 2007; Bozzo et al., 2010, 2012; Drave et al., 2014). Overall, these sources appear to be substantially sub-luminous compared to the classical SgXBs (Bozzo et al., 2015).

Given several similarities in the spectral shape between SFXTs (especially when in outburst) and classical $\mathrm{SgXBs}$, it has always been assumed that the bulk of the X-ray emission from the two classes of systems has a common origin and should be produced by the accretion of the structured supergiant star wind onto the neutron star. Clumps are thus expected to drive at least part of the X-ray variability of the SFXTs. However, the physical mechanisms responsible for the more extreme behaviour of these sources is still not fully understood and remains highly debated (Sidoli, 2012; Paizis and Sidoli, 2014; Walter et al., 2015). We discuss this issue more extensively in Sect.4.5

4.1 X-ray variability and clumpy stellar winds: flares and off-states

It was shown (see Sect. 2.2.1) that X-ray luminosity $L_{X}$ recorded from $S g X B$ s and SFXTs traces reasonably well the mass per unit time that is accreted by the neutron star. It was also shown in that section how changes in the mass accretion rate of a wind-fed system can lead to almost immediate variations of its X-ray luminosity, as there is no accretion disk mediating the transfer of material from the supergiant companion to the compact object. In the simplest accretion scenario depicted in Sect.2.2.1. where all complications related to the presence of the neutron star magnetic field and spin rotation are neglected, we can obtain an 
expression connecting directly the X-ray luminosity with the properties of the stellar wind merging Eq. 13 and 17

$$
L_{\mathrm{X}}=4 \pi \zeta \rho(a, t) \frac{\left(G M_{\mathrm{NS}}\right)^{3}}{R_{\mathrm{NS}} v_{\mathrm{w}}^{3}},
$$

where we also applied the simplification $v_{\mathrm{rel}} \sim v_{\mathrm{w}}$. From this equation, it can be seen that a change in the density of the wind can lead to a comparable variation of the X-ray luminosity and the latter can be boosted even more in case of variations in the wind velocity. As we have seen in Sect. 2.1.2 changes in density and velocity of the wind at the neutron star location can be induced by the presence of moderately dense clumps or inhomogeneities. It thus turns out from Eq. 54 that a dynamic range up to 10-100 in the X-ray luminosity of wind-fed binaries can be easily achieved by assuming that the neutron star is accreting from a clumpy and structured wind. It is worth remarking here that Eq. 54 is derived in the simplest case of the direct accretion regime, thus neglecting any factor that might arise due to the presence of a centrifugal/magnetic gate and the onset of a possible settling accretion regime.

Under the assumptions above, one could thus use the observed X-ray variability and continuous flaring behaviour in classical $\mathrm{SgXBs}$ to estimate the properties of clumps needed to regulate it. In the simplified approach proposed by Walter and Zurita Heras (2007), it is assumed that the neutron star accretes only a certain fraction of the clump mass $\left(M_{\mathrm{cl}} \gtrsim M_{\mathrm{acc}}\right)$ and that its radius is larger than the NS corotation radius $\left(R_{\mathrm{cl}} \gtrsim R_{\mathrm{acc}}\right.$; see Eq. 14). In this case we can define:

$$
M_{\mathrm{cl}}=\left(R_{\mathrm{cl}} / R_{\mathrm{acc}}\right)^{2} M_{\mathrm{acc}} .
$$

If we further consider that the duration of a flare $t_{\mathrm{fl}}$ is proportional to the time that the neutron star needs to go through the clump and accrete (at least) a part of it, we can estimate

$$
R_{\mathrm{cl}} \sim v_{\mathrm{fl}} \times t_{\mathrm{fl}} .
$$

Here the velocity of the clump $v_{\mathrm{fl}}=f v_{\mathrm{w}}$ is assumed to be a fraction $f$ of the stellar wind velocity $v_{\mathrm{w}}$. The mass of the clump is thus given by:

$$
M_{\mathrm{cl}}=\frac{L_{\mathrm{X}} R_{\mathrm{NS}}}{\left(G M_{\mathrm{NS}}\right)^{3}} t_{\mathrm{fl}}^{3} f^{2} v_{\mathrm{w}}^{6} .
$$

For typical parameters of flares in classical $\mathrm{SgXBs}$ (see, e.g., the right panel of Fig. 11) and considering a standard neutron $\operatorname{star}\left(R_{\mathrm{NS}}=10 \mathrm{~km}, M_{\mathrm{NS}}=1.4 M_{\odot}\right)$, one obtains:

$$
M_{\mathrm{cl}} \simeq 5 \times 10^{18} \mathrm{~g}\left(\frac{L_{\mathrm{X}}}{10^{36} \mathrm{erg} / \mathrm{s}}\right)\left(\frac{t_{\mathrm{fl}}}{0.5 \mathrm{ks}}\right)^{3}\left(\frac{f}{0.5}\right)^{2}\left(\frac{v_{\mathrm{w}}}{1000 \mathrm{~km} \mathrm{~s}^{-1}}\right)^{6}
$$

that yields a reasonable value compared to the clump mass of $\sim 10^{18} \mathrm{~g}$ (at the most) inferred from LDI numerical simulations of hot star winds (see Sect. 2.1.2). Walter and Zurita Heras (2007) also noted that through Eq. 58 particularly long $\left(t_{\mathrm{fl}} \gg 1 \mathrm{ks}\right)$ and structured flares could still be interpreted in terms of accretion from an inhomogeneous wind, if each individual peak of intensity during the flare is associated with the accretion of a small clump being part of a clustered structure.

Refined calculations to link X-ray flares to the physical properties of the clumps were presented by Ducci et al. (2009), including the orbital characteristics of SgXBs and a distribution in radius and mass for the clumps. The effect of different impact parameters for 
the clump accretion onto the neutron star was also included into the calculation, thus permitting more reliable estimates for the clump physical properties from the observation of $\mathrm{X}$-ray flares that those inferred from the simplified calculations presented above. Assuming the standard direct accretion scenario (Sect. 2.2.2), Ducci et al. (2009) derived synthetic Xray lightcurves of several classical $\mathrm{SgXBs}$, and comparing these results with those obtained from X-ray observations, inferred ranges for the clump masses of $5 \times 10^{18} \mathrm{~g}$ to $5 \times 10^{21} \mathrm{~g}$ in Vela X-1, $5 \times 10^{16} \mathrm{~g}$ to $2 \times 10^{19} \mathrm{~g}$ in $4 \mathrm{U} 1700-37$, and $5 \times 10^{16} \mathrm{~g}$ to $2 \times 10^{21} \mathrm{~g}$ in IGR J164184532 (see also Romano et al., 2012). In some cases, these calculations thus suggest the presence of clumps with a significantly larger mass than that expected according to LDI simulations of massive star winds (see above).

Similar results were also independently obtained by other authors with different methods. Fürst et al. (2010) studied the lightcurve of Vela X-1 as observed by the INTEGRAL satellite and showed that the log-normal distribution of the brightness of individual flares could be well reproduced in a simulation in which the direct accretion process onto the neutron star is taking place from a clumpy rather than a smooth stellar wind. They found that clumps in Vela X-1 should have masses in the range $5 \times 10^{19}-10^{21} \mathrm{~g}$ in order to explain the Xray data (see also Fürst et al., 2014). Similarly, Martínez-Núñez et al. (2014) and Fürst et al. (2014) could only explain the properties of some X-ray flares detected during dedicated observations of Vela X-1 by assuming clump masses of $\gtrsim 10^{21} \mathrm{~g}$.

On one hand, it thus seems that the scenario of the accretion from a clumpy wind can explain reasonably well the X-ray variability of classical $\mathrm{SgXBs}$. In particular, it can reproduce the main features of their high energy emission and the typical shape of the observed lightcurves. On the other hand, a number of published results suggest the presence of a population of clumps in these sources that are a factor of $\sim 100-1000$ more massive than those expected from the LDI simulations (see Sect.2.1.2. One possibility is that such a discrepancy arises as a consequence of the currently known limitations of LDI simulations, which cannot yet account for complex interactions between these structures when multi-dimensional approaches are used (and might thus lead to the formation of larger structures than currently foreseen; see Sect.(2.1.2). However, it is also likely that the presence of centrifugal/magnetic gates or the onset of a settling accretion regime could play an important role in the determining the release of the X-ray luminosity, thus affecting the estimates of the clump masses derived with the techniques described above. As shown in Sect. 2.2.2, the switch between one regime of accretion to another can boost the variation of the system X-ray luminosity dramatically, even in case of modest changes in the wind density and/or velocity. In these cases, the X-ray luminosity is no longer an obvious tracer of the mass accretion rate, and small clumps can easily become the cause of large swings in the X-ray emission properties of the system. Neglecting these effects can thus potentially led to an overestimate the sizes and masses of clumps. A more quantitative analysis is currently hampered by the uncertainties on the physical processes occurring in the different accretion regimes and the poorly known properties of the neutron stars hosted in many of the $\mathrm{SgXBs}$. The development of a clumpy wind accretion model accounting for different accretion regimes is still under way.

Clumpy wind accretion models encounter more difficulties in explaining the extreme variability of the SFXT sources. By looking at Eq. 54 one would immediately conclude that unreasonably large and dense clumps would be needed to achieve a dynamic range in the X-ray luminosity comparable to that of the SFXTs (up to $10^{5}-10^{6}$ ). Furthermore, the typical lightcurve of an SFXT is significantly different from that of a classical system, as it is characterized by sporadic isolated bright flares between which the source spend a long time in a much lower and less variable luminosity regime (see the right panel of Fig 11). As we discuss in Sect. 4.5, the models proposed so far to interpret the peculiar behaviour of the 
SFXTs are considering a number of different mechanisms to regulate their variability, but in all cases clumps still play an important role in achieving the overall $\mathrm{X}$-ray dynamic range.

Convincing evidences for the presence of clumps in the stellar winds of SFXT supergiants have been found especially by searching for absorption events in the soft X-ray lightcurves of these objects. The role of dense clumps is indeed two-fold. Clumps that lead to an increased mass accretion rate produce X-ray flares as discussed above, but during the ingresses (egresses) from these flares the clump approaching (moving away from) the neutron star will also cause a dimming or even obscuration of the X-ray source. These events lead to remarkable increases of the absorption column density in the direction to the sources and are expected to be even more frequent than the X-ray flares (all clumps simply passing through the line of sight to the observer even without being accreted contribute to increase the $N_{\mathrm{H}}$ ). If we use again the simple equations presented earlier in this section, we can estimate the absorption column density associated to the passage of a clump along the line of sight to the observer as:

$$
\begin{aligned}
& N_{\mathrm{H}} \simeq \frac{M_{\mathrm{cl}}}{R_{\mathrm{cl}}^{2} m_{\mathrm{p}}}=\frac{L_{\mathrm{X}} R_{\mathrm{NS}}}{\left(G M_{\mathrm{NS}}\right)^{3}} t_{\mathrm{fl}} v_{\mathrm{w}}^{4}= \\
& 9 \times 10^{22} \mathrm{~cm}^{-2}\left(\frac{L_{\mathrm{fl}}}{10^{36} \mathrm{erg} \mathrm{s}^{-1}} \frac{t_{\mathrm{fl}}}{0.5 \mathrm{ks}}\left(\frac{v_{\mathrm{w}}}{1000 \mathrm{~km} \mathrm{~s}^{-1}}\right)^{4}\right)
\end{aligned}
$$

From Eq. 59 we note that clumps usually remain optically thin in the X-rays (see also Sect. 3.9) and that the increase in the absorption column density is in the range fully accessible through soft X-ray observations (e.g. carried out with XMM-Newton, Swift/XRT, Suzaku, and Chandra). Rampy et al. (2009) were the first to report the evidence of clumps in the winds of the SFXTs supergiant companions by using this technique. These authors detected an absorption events in the Suzaku lightcurve of the SFXT IGR J17544-2619 that lasted $300 \mathrm{~s}$ and estimated that such effect could have been produced by a clump as large as $\sim 4.2 \times 10^{9} \mathrm{~cm}$ and with a mass of $\sim 1.5 \times 10^{18} \mathrm{~g}$. The increase of the absorption column density in the direction of the SFXT IGR J18410-0535 during a bright flare from the source led Bozzo et al. (2011) to conclude that the event could have been caused by a clump with $R_{\mathrm{cl}}$ $\sim 8 \times 10^{11} \mathrm{~cm}$ and $M_{\mathrm{cl}} \sim 1.4 \times 10^{22} \mathrm{~g}$. By applying the refined clumpy wind accretion model developed by Ducci et al. (2009) to the SFXTs IGR J11215-5952 and IGR J18483-0311, clumps in the mass range $10^{17} \mathrm{~g}$ to $5 \times 10^{20} \mathrm{~g}$ and $10^{18} \mathrm{~g}$ to $5 \times 10^{21} \mathrm{~g}$ were obtained (see also Romano et al., 2010).

As for the classical $\mathrm{SgXBs}$, the estimated properties of the clumps presented above were all obtained by assuming the simplest direct accretion regime and neglecting possible systematic uncertainties affecting the luminosity and absorption column density determined from the fit to the X-ray spectra of these sources. For this reason, caution should be taken when comparing the derived clump properties in the SFXTs with those inferred from simulations and observations of isolated supergiant stars.

Furthermore, it should be noted that in both cases in which clump parameters are derived from flares of $\mathrm{SgXB}$ sources or absorption events in their lightcurves, there are systematic uncertainties on the values of the X-ray luminosity, $L_{\mathrm{X}}$, and the amount of absorbing neutral material, $N_{\mathrm{H}}$, that are usually not included in the calculations. We discuss separately these issues in Sect.4.4.1 and 4.4.2

X-ray flares are not the only way in which we can probe the presence of small scale structures in the winds of supergiant stars in $\mathrm{SgXBs}$. At least four classical $\mathrm{SgXBs}$ (Vela X- 
1, 4U 1700-329, 4U 1907+09 and GX 301-2) show occasional "off-states" (see Table 3), which have been often interpreted in terms of the clumpy properties of the stellar wind. During the off-states the X-ray luminosity of these sources reaches very low levels, often below the observable limit, and sometimes also other changes to the X-ray emission properties are detected. It is important to note here that different works in the literature use the term off-state for different levels of luminosity even in the same source, as the effective limit is often driven by the sensitivity and energy range of the observing instrument rather than by physical considerations. A variety of mechanisms has been proposed to explain these low luminosity events, and we summarise below all relevant findings from the literature.

As in many other areas, the best studied source for low and off-states is Vela X-1, due to its relatively small distance and high X-ray flux (see Table 2). The possibly first observation of such a state was reported by Kallman and White (1982), and described as a "brief absorption event" lasting for $\sim 6$ minutes with no evident change in the source spectral properties. In 1983, a Tenma observation showed a drop in intensity lasting for less than 80 minutes and during which pulsations disappeared as well. Hayakawa (1984) discussed both of the preceding observations as being possibly caused by a planet eclipsing the source, while Inoue et al. (1984), analysing the 1983 observation in detail offered a sudden choking of the accretion as alternative. Lapshov et al. (1992) reported on an extended low state in Vela X-1 of more than 10 hours duration outside the source X-ray eclipse observed by Granat/Watch. In this case the off-state was explained in terms of an abrupt reduction in the accretion rate. At the start of a RXTE/PCA observation in 1996, Kreykenbohm et al. (1999) noted an interval of at least $550 \mathrm{~s}$ duration with low count rates and no observable pulsations from the source. In a somewhat different case, Kretschmar et al. (2000) found a sudden flux decrease in January 1998 with RXTE with disappearing pulsations while significant non-pulsed source flux remained for more than an hour. As an explanation, a massive $(\tau \approx 1.6)$ and very large $\left(\sim 10^{13} \mathrm{~cm}\right)$ clump obscuring the pulsar and destroying coherent pulsations by scattering was considered. Using deep INTEGRAL observations of Vela X-1 from late 2003 Kreykenbohm et al. (2008) found five occurrences of off-states with durations between 520 and $1990 \mathrm{~s}$ (an example of an off-state from Vela X-1 is shown in Fig. 13). For four of the five instances the preferred explanation is a combination of strong density fluctuations in the wind and the onset of a centrifugal gate, while for the last and longest, again a massive clump was found to best explain the data. Doroshenko et al. (2011) analysed a $100 \mathrm{ks}$ observation of Vela X-1 by Suzaku and found three off-states. At odds with previous results, mostly obtained with less sensitive instruments, they found that pulsations continued also during the off-states, albeit with clear changes in the spectra and pulse profiles. They explained their results by invoking a gated accretion, in which the magnetospheric boundary becomes stable with respect to the RTI but remains unstable with respect to the KHI (that usually allows accretion to occur at a somewhat reduced rate; see Sect. 2.2.2). Sidoli et al. (2015) combined data over 10 years of INTEGRAL observations searching for low or off-states in the hard X-ray lightcurve of Vela X-1. They found that while some off-states can appear at any orbital phase, other are more common close to the eclipses and with different distributions for the eclipse egress and ingress. This asymmetric distribution is explained by scattering in ionized material, compatible with the photoionisation wake (see, e.g., the geometry in the Vela X-1 system outlined by Kaper et al. (1994)). The cases of off-states evenly distributed around the orbit could be caused either by exceptionally low matter density or gated accretion or by changes in accretion regime within the spherical settling accretion model (Shakura et al., 2013). In order

\footnotetext{
29 A source that we do not deeply discuss in this review as the nature of its accreting compact object is still debated.
} 


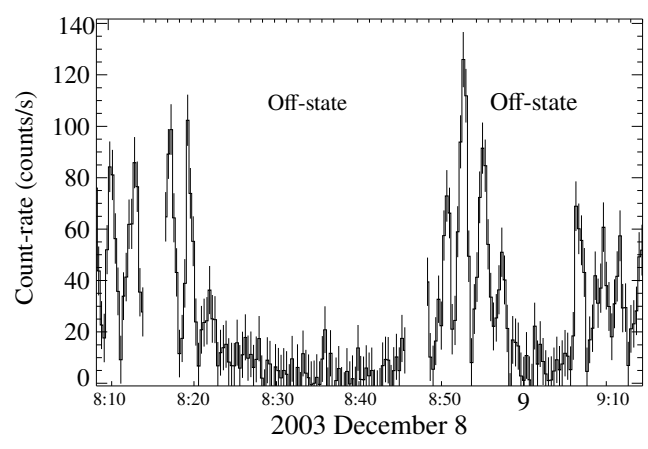

Fig. 13 An example of an off-state in Vela X-1 as observed by IBIS/ISGRI onboard INTEGRAL (20-60 keV). Due to the relatively low sensitivity of ISGRI to faint sources, no residual flux from Vela X-1 is recorded by INTEGRAL during these offstates. However, more sensitive instruments revealed that the source is still emitting in $\mathrm{X}$-rays and pulsating during these events, albeit at a much more reduced level than usual (see text for details). Reproduced with permission from Kreykenbohm et al. 2008).

to test possible explanations of the observed off-states, Manousakis and Walter (2015) created a hydrodynamical model of the Vela X-1 system. They found that low-density bubbles formed close to the neutron star, due to unstable hydrodynamic flows, lead to off-states with typical durations between 5-120 minutes. As in the simulations presented by these authors the stellar wind is assumed to follow the simplest CAK approximation (see Sect. 2.1.1), these findings opened the new interesting possibility that (at least) not all off-states could be related to clumps and/or gated accretion regimes.

In the case of $4 \mathrm{U} 1907+09$, a less deeply studied source than Vela X-1, in't Zand et al. (1997) first noted a peculiar dipping behaviour in several RXTE observations, finding at least 11 dips longer (up to $\sim 1.5 \mathrm{~h}$ ) than the pulse period ( $\sim 600 \mathrm{~s}$ ). Voids in the inhomogeneous medium surrounding the neutron star were considered the most likely possibility to interpret the drops in the accretion, and the possibility was considered that voids might also be created due to photoionization. Roberts et al. (2001) found 4U 1907+09 in a very low state during two out of the four performed ASCA observations spread roughly equally around the $8.4 \mathrm{~d}$ orbital period. These low states of relatively long duration $(\sim 10 \mathrm{ks})$ showed no signs of increased absorption, and were interpreted in terms of magnetically inhibited accretion. Rivers et al. (2010) noted dips in the Suzaku observations of 4U 1907+09, which Doroshenko et al. (2012) analysed later in more detail. These observations included four off-states of about 700-900 s duration. Similar to the case of Vela X-1, Doroshenko et al. (2011) demonstrated the presence of a weak but significant flux and pulsations during all these off-states and interpreted them in a coherent way for the two sources.

GX 301-2 is a rather peculiar system with an eccentric orbit $(e=0.462)$ and a marked orbital flux variation with a strong pre-periastron flare. Göğüs et al. (2011) noted a peculiar dip in one of the RXTE lightcurve of the source during which X-ray pulsations declined, disappeared for one spin cycle (686 s), and then reappeared again. Spectral changes were similar to those observed during the dips in Vela X-1 (Kreykenbohm et al., 1999). A brief cessation of accretion to the magnetic poles was considered the most probable explanation. During an observation of part of the source pre-periastron flare with XMM-Newton, Fürst et al. (2011) found another interval of low flux in which the pulsations ceased almost completely for several spin cycles, without any indication of increased absorption or other spectral changes. Investigating this dip in detail, they argued that it is most likely that during the dip the accretion ceased and the afterglow of fluorescent iron around the neutron star accounted for the main portion of the residual X-ray flux.

Two possible off-states were also identified in the lightcurves of the classical $\mathrm{SgXB}$ IGR J16418-4532 and the SFXT IGR J16328-4726 by Drave et al. (2014) and Bozzo et al. (2012), respectively. In both cases the statistics of the X-ray data was far too low to perform a 
Table 3 Overview of off-state or low flux observations in SgXBs. In some cases luminosities have been estimated based on the information in the original work and the newer distance estimates became available in the literature (see Table 2.

\begin{tabular}{|c|c|c|c|c|c|}
\hline Source & Date & Instrum. & Energy range & Off-state luminosity & References \\
\hline$\overline{\text { Vela X-1 }}$ & 9 May 1979 & Einstein & $2-10 \mathrm{keV}$ & not quantified & $\overline{\mathrm{KW} 82, \mathrm{Ha} 84}$ \\
\hline Vela X-1 & 12 Mar 1983 & Tenma & $3-9 \mathrm{keV}$ & $<10 \%$ of preceding & In84, Ha84 \\
\hline Vela X-1 & 9 Jan 1991 & Watch & $8-15 \mathrm{keV}$ & not quantified & $\mathrm{La} 92$ \\
\hline Vela X-1 & 23 Feb 1996 & RXTE/PCA & $3-30 \mathrm{keV}$ & $<15 \%$ of normal & Kr99 \\
\hline Vela X-1 & 22 Jan 1998 & RXTE/PCA & $2-60 \mathrm{keV}$ & not quantified & $\mathrm{Kr} 00$ \\
\hline Vela X-1 & 8 Dec 2003 & ISGRI & $20-40 \mathrm{keV}$ & not quantified & $\mathrm{Kr} 08$ \\
\hline Vela X-1 & 17-18 Jun 2008 & Suzaku/XIS & $0.4-70 \mathrm{keV}$ & $\sim 2.4 \times 10^{35} \mathrm{erg} \mathrm{s}^{-1}$ & Do11 \\
\hline Vela X-1 & 2002-2012 & ISGRI & $22-50 \mathrm{keV}$ & $\lesssim 3 \times 10^{35} \mathrm{erg} \mathrm{s}^{-1}$ & Si15 \\
\hline 4U 1907+09 & 17-23 Feb 1996 & RXTE/PCA & $2-15 \mathrm{keV}$ & up to $98 \%$ decrease & iZ97 \\
\hline 4U 1907+09 & $14-16$ Oct 1996 & ASCA & $2-10 \mathrm{keV}$ & $\sim 1.6 \times 10^{34} \mathrm{erg} \mathrm{s}^{-1}$ & Ro01 \\
\hline 4U 1907+09 & 2-3 May 2006 & Suzaku/XIS & & $\sim 10^{35} \mathrm{erg} \mathrm{s}^{-1}$ & Ri10, Do12 \\
\hline $4 \mathrm{U} 1700-37$ & 17-18 Feb 2001 & XMM-Newton & $0.5-10 \mathrm{keV}$ & $2 \times 10^{35} \mathrm{erg} \mathrm{s}^{-1}$ & vdM05 \\
\hline$\overline{\mathrm{GX} 301-2}$ & 28 May 2010 & RXTE/PCA & $3-25 \mathrm{keV}$ & not quantified & Go11 \\
\hline GX 301-2 & & XMM-Newton & $2-10 \mathrm{keV}$ & $\lesssim 4 \times 10^{35} \mathrm{erg} \mathrm{s}^{-1}$ & Fu11 \\
\hline$\overline{\text { IGR J16418-4532 }}$ & & XMM-Newton & $0.5-10 \mathrm{keV}$ & $\lesssim 8 \times 10^{34} \mathrm{erg} \mathrm{s}^{-1}$ & $\overline{D r 13}$ \\
\hline IGR J16328-4726 & & XMM-Newton & $0.5-10 \mathrm{keV}$ & $4.1 \times 10^{35} \mathrm{erg} \mathrm{s}^{-1}$ & $\overline{B o 12}$ \\
\hline IGR J17544-2619 & 16 Sept 2012 & XMM-Newton & $0.5-10 \mathrm{keV}$ & $4 \times 10^{32} \mathrm{erg} \mathrm{s}^{-1}$ & Dr14 \\
\hline
\end{tabular}

$\begin{array}{lll}\text { Bo12: Bozzo et al. (2012) } & \text { Ha84: Hayakawa (1984) } & \text { La92: Lapshov et al. (1992) } \\ \text { Do11: Doroshenko et al. (2011) } & \text { In84: Inoue et al. (1984) } & \text { Ri10 Rivers et al. (2010) } \\ \text { Do12: Doroshenko et al. (2012) } & \text { iZ97: in’t Zand et al. (1997) } & \text { Ro01 Roberts et al. (2001) } \\ \text { Dr13: Drave et al. (2013b) } & \text { KW82: Kallman and White (1982) } & \text { Si15: Sidoli et al. (2015) } \\ \text { Dr14: Drave et al. (2014) } & \text { Kr00: Kretschmar et al. (2000) } & \text { vdM05: van der Meer et al. (2005) } \\ \text { Fu11: Fürst et al. (2011) } & \text { Kr99: Kreykenbohm et al. (1999) } & \\ \text { Go11: Göğüs et al. (2011) } & \text { Kr08: Kreykenbohm et al. (2008) }\end{array}$

detailed spectral and timing analysis of the two events, but Table 3 shows that the luminosity measured during the off-state was roughly comparable to those obtained in the brighter SgXBs mentioned above.

Although the origin of the off-states is still unclear and highly debated, all possibilities described above are particularly interesting to use these events as additional probes of stellar winds in $\mathrm{SgXBs}$. If off-states are due to voids between clumps, observations of these events could be used in combination with the detection of X-ray flares to build a more complete map of the stellar wind and compare this with the outcomes of LDI or more advanced simulations (see Sect. 2.1.2). A complication to this simple scenario comes from the fact that, as pointed out by several authors, off-states might also be (at least) partly related to a change in the accretion regime, or to the onset of a centrifugal and/or magnetic inhibition of accretion, or to the scattering effect produced by photo-ionised large-scale wind structures. It is particularly tricky to disentangle these effects, because any drop in the mass accretion rate implies an increase in the size of the neutron star magnetosphere and thus a large possibility to switch toward a propeller-like accretion regime (see Sect.2.2.2). Additionally, as for the $\mathrm{X}$-ray flares discussed above, the properties of the stellar winds inferred from the study of the off-states would also be affected by the known systematic uncertainties on the source luminosity and absorbing material derived from the X-ray data (see Sect.4.4.1 and 4.4.2). 
4.2 Disrupting the stellar wind: photoionization, accretion wakes, and super-orbital modulations

Observations and hydrodynamic simulations of wind-fed systems have convincingly shown that these stellar winds are heavily affected by the X-ray radiation emitted by the neutron star (see, e.g., Blondin et al., 1991, and references therein). If the luminosity of the X-ray source is sufficiently intense (typically $\gtrsim 10^{35}-10^{36} \mathrm{erg} \mathrm{s}^{-1}$ ), the photoionization of the wind changes the state of a large fraction of the heavy ions within the so-called Strömgren sphere (they become ionized to a higher degree, with corresponding lines only in the high energy, low-flux spectral region of the star), and the driving acceleration mechanism of the wind is cut off (see Sect. 2.1). The wind is not further accelerated, it is accumulated at the neutron star location, giving rise to a "photoionization wake" (see, e.g., Fransson and Fabian, 1980). As the neutron star moves along its orbit, the photoionization wake is displaced together with the compact object and the additional material halted at the bow-shock (see Sect.2.2.1). During the neutron star revolution, the latter give rise to an elongated dense structure that is usually termed "accretion wake" (see, e.g., Blondin et al., 1990). A substantial theoretical effort was devoted in the past to calculate the details of the interaction between the X-ray radiation and the surrounding environment, starting from the pioneering works of Tarter et al. (1969) and Krolik and Kallman (1984) and arriving to the more specialized calculations of Friend and Castor (1982) and Ho and Arons (1987) to the case of wind-fed binaries (see also, more recently, Ducci et al. (2010)). However, only with the most recent hydrodynamical simulations it has been possible to follow in detail the photoionization of the wind (see, e.g., Krtička et al., 2012; Krticka et al., 2015) and the formation of time-dependent accretion/photoionization wakes (Manousakis and Walter, 2015; Čechura and Hadrava, 2015).

As we discuss below, the photoionization of the wind and the formation of accretion/photoionization wakes are all additional tools to probe the characteristics of the stellar winds in SgXBs. In particular, they can be used to indirectly infer the physical properties of the unperturbed winds in these systems by comparing observational results with numerical simulations of the interaction between the supergiant outflow and the X-ray radiation from the compact object.

Among the different spectral features produced by the photoionization of the stellar wind and thus arising especially in the photoionization wake, the Lyman series lines from the $\mathrm{H}$ and He-like ions are the most appropriate features to probe the dynamics of this medium. These lines are formed by radiative decays following either photoexicitation or radiative recombination, and their properties can be accurately calculated from theory. Shifts in the centroid energies of these lines can thus be easily evaluated from X-ray observations, providing a direct measurement of the wind velocity. Together with these lines, the width in energy of the radiative recombination continuum, which is formed by the photons generated in recombination and distributed into a continuum (typically line-like in the case of the $\mathrm{SgXBs}$ ), provides an estimate of the wind electron temperature. These diagnostic techniques have been successfully exploited, as an example, in the case of Vela X-1 by Watanabe et al. (2006). The red and blue velocity shifts measured at different orbital phases from the emission lines of several elements ( $\mathrm{Si}, \mathrm{S}, \mathrm{Ca}, \mathrm{Al}, \mathrm{Mg}, \mathrm{Ne}$ ) at energies $\sim 1.9-2.7 \mathrm{keV}$ revealed that the supergiant wind close to the neutron star location has a velocity that is a factor of a few lower than $\sim 2-3$ expected. By using Monte Carlo simulations, Watanabe et al. (2006) concluded that the reduced velocities were due to the effect of the wind photoionization by the neutron star X-ray emission that led to a substantial drop in the radiatively driven acceleration of the stellar wind. The parameters of the unperturbed wind reconstructed from their Monte Carlo simulations provided a mass-loss rate of $(1.5-2.0) \times 10^{-6} M_{\odot} \mathrm{yr}^{-1}$ and 
a terminal wind velocity of $1100 \mathrm{~km} \mathrm{~s}^{-1}$, compatible with independent estimates obtained from the P Cygni profile of UV resonance lines and expected for a B0.5 Ib supergiant (as the one hosted in Vela X-1; see Table 2). Similar results have been presented also for the bright $4 \mathrm{U} 1700-37$ system, a source that we do not deeply discuss in this review as the nature of the accreting compact object in this system is still debated (Boroson et al., 2003).

In general, despite the interest and the important achievements of such studies, they have been carried out so far only for a few close-by SgXBs. This is mainly due to the fact that $\mathrm{SgXBs}$ are heavily absorbed in the soft X-ray domain and the sensitivity of the currently available $\mathrm{X}$-ray gratings, the sole instruments that permit to analyse spectral lines at $\lesssim 3 \mathrm{keV}$ with the required accuracy, limits the possibility to carry out these studies only to tentatively bright objects.

The presence of an accretion wake can be observationally inferred in many sources by studying the different shapes of the energy resolved lightcurve folded on the system orbital period (see, e.g., Falanga et al., 2015, for examples), or by measuring the variation of the $N_{\mathrm{H}}$ at different orbital phases. The absorption column density can easily reach values as high as $N_{\mathrm{H}} \gtrsim 10^{23}-10^{24} \mathrm{~cm}^{-2}$ when the accretion wake is located along the line of sight to the observer. In a system where the wind can be described by a $\beta$-law, it follows the CAK approximation (Sect.2.1), the hydrogen number density $n_{\mathrm{H}}(r)$ as a function of the distance $r$ from the supergiant is given by:

$$
n_{\mathrm{H}}(r)=\frac{X_{\mathrm{H}} \dot{M}_{\mathrm{w}}}{m_{\mathrm{H}} v_{\infty}\left(1-R_{\star} / r\right)^{\beta} 4 \pi r^{2}}
$$

Here $X_{\mathrm{H}}$ is the hydrogen mass fraction, $\dot{M}_{\mathrm{w}}$ is the supergiant mass loss rate, $v_{\infty}$ the terminal velocity of its wind and $R_{\star}$ its radius. The corresponding value of the $N_{\mathrm{H}}$ can be calculated by integrating $n_{\mathrm{H}}(r)$ along the observer line of sight and depends on the system inclination $i$, its semi-major axis $a$, its eccentricity $e$, and the longitude of its periastron $\omega$. Any deviation in the measured profile of the $N_{\mathrm{H}}$ compared to the one in Eq. 60, can be ascribed to the presence of massive and long-lived structure in the system, and thus most likely to an accretion wake. We show an application of this method in Fig. 14 Note that the observations used to study the orbital profile of the absorption column density in a system are usually collected over time scales much longer than the orbital period and thus the $N_{\mathrm{H}}$ enhancement cannot be caused by clumps or other short lived structures. It is indeed expected that the contribution of these fast moving structures is averaged away by the long integration times.

As the characteristics of the accretion wake depend on the compact object and wind properties, some authors have been using measurements of the averaged absorption column density profile to indirectly infer the supergiant mass low rate and the wind velocity from the comparison between observational results and numerical simulations (i.e., as done in the case of the emission lines from photoionized plasmas described above). This technique has been applied, for example, to estimate the wind velocity of the classical SgXB IGR J172523616 (Manousakis and Walter, 2011).

A relatively recent and puzzling discovery is the detection in several $\mathrm{SgXBs}$ of a superorbital variability, i.e., a modulation of their X-ray flux on a time-scale that is significantly longer that the orbital period. The super-orbital variability is a well-known phenomenon of disk-accreting systems and it is usually ascribed to the presence of a precessing warp in the disk that periodically obscures the X-ray source (Ogilvie and Dubus, 2001). As in all SgXBs of interest for this review accretion takes place from the stellar wind, it could be possible that the periodical obscuration of the X-ray source is related to other dense structures, e.g., the accretion wake. However, Farrell et al. (2008) showed that at least in the case of the 


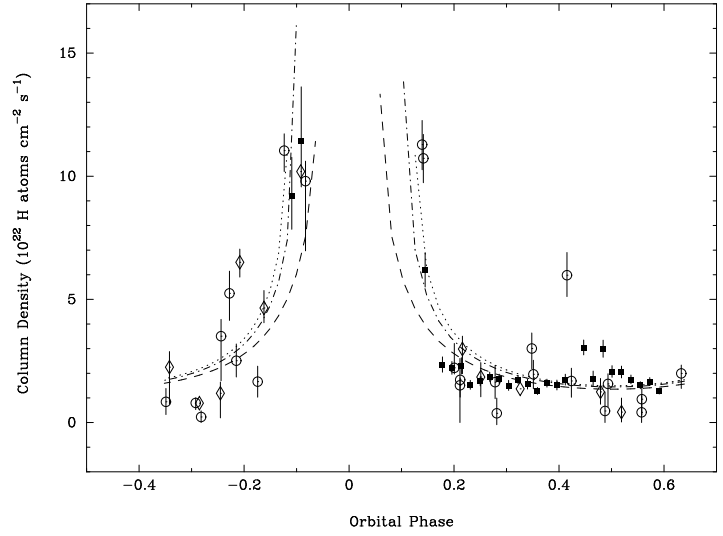

Fig. 14 Orbital modulation of the $N_{\mathrm{H}}$ as a function of the orbital phase in the eclipsing $\mathrm{SgXB} 4 \mathrm{U}$ 1538-522, (see Mukheriee et al., 2006, reproduced with permission). The authors assume a circular orbit with $a_{x} \sin (i)=53.1 \quad$ lt-s and three different values for the inclination: $i=65^{\circ}, 75^{\circ}, 85^{\circ}$ (dashed, dashed-dotted, and dotted lines respectively). The following wind parameters are also assumed: $\beta=0.5$ (Please note that the assuming $\beta$ is too small, since considering the so-called finite-cone-angle effect implies a minimum $\beta$ value of $\sim 0.7-0.8$ ), $\dot{M}=10^{-6} M_{\odot} / y r, v_{\infty}=1000 \mathrm{~km} / \mathrm{s}$. They use observations from RXTE in 1997 (diamonds), BeppoSAX in 1998 (squares), and RXTE in 2003 (circles).

classical SgXB 1A 0114+650, no significant changes of the absorption column density in the direction of the source could be measured at different super-orbital phases. These authors suggested that a modulation of the accretion rate on the super-orbital time-scale could better explain the data, but so far no mechanism has been proposed to drive such changes. Corbet and Krimm (2013) reported on several additional discoveries of super-orbital modulations both among the classical SgXBs as well as in SFXTs. In all these systems, there is a clear correlation between the orbital and the super-orbital periods, and thus it was suggested that the mechanism modulating the mass accretion rate should be related to the separation between the compact object and the supergiant star. As the mass accretion rate in all relevant systems is regulated by the wind of the massive star and only a few systems among both the classical sgXBs and SFXTs show superorbital modulations, it is likely that the involved mechanisms are related to some specific properties of the mass donor star rather than of the compact object. An example is the presence of an off-set between the orbital plane and the rotation axis of the primary, which could modulate the rate at which the stellar wind arrives close to the compact object on a time scale related (but longer) that the orbital period of the system. The presence of an (even small) eccentricity could affect and complicate the period of the resulting super-orbital modulation by enhancing the mass accretion rate toward periastron and decreasing it at apastron (see, Corbet and Krimm, 2013, and references therein).

4.3 Fluorescence emission lines from the stellar wind and the neutron star surroundings

An important emission line formation process in wind-fed systems is the fluorescence from ions in a low charge state, produced due to the illumination by the X-ray source of the circumstellar material. As the intensity of a fluorescence emission is proportional to the fluorescence yield and the latter increases with the atomic number of the ion, the $\mathrm{Fe} \mathrm{K} \alpha$ fluorescent line is usually the most prominent among these features in the spectra of $\mathrm{SgXBs}$ 

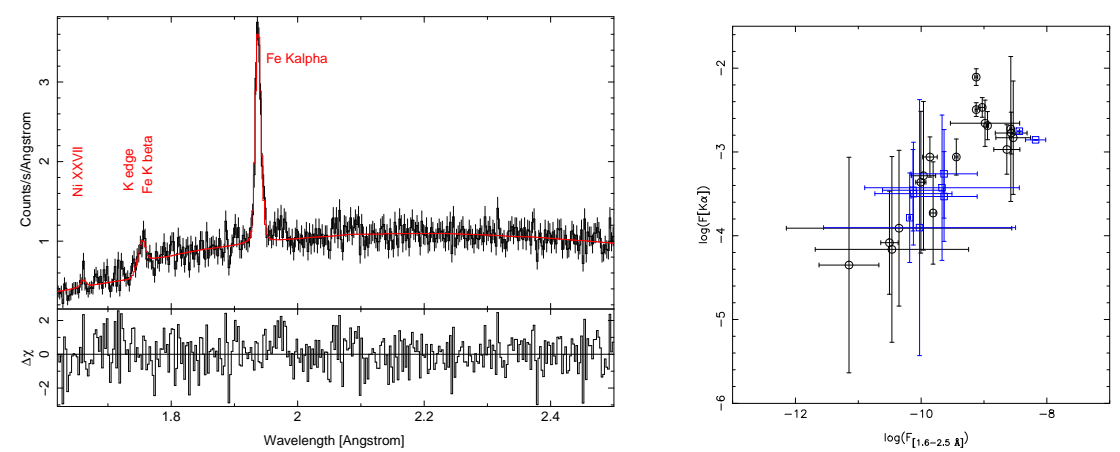

Fig. 15 Left: plot reproduced with permission from Torrejón et al. (2010a) . Chandra HETG spectrum of the HMXB Vela X-1. We can see a prominent $\mathrm{Fe} \mathrm{K} \alpha$ line together with $\mathrm{FeK} \beta$. Right: plot reproduced with permission from Torreión et al. (2010a). The logarithm of the flux of $\mathrm{Fe} \mathrm{K \alpha}\left(\mathrm{ph} / \mathrm{cm}^{2} / \mathrm{s}\right)$ is well correlated with the logarithm of the continuum flux $\left(\mathrm{erg} / \mathrm{cm}^{2} / \mathrm{s}\right)$. The black circles correspond to HMXBs, while blue squares indicate low mass X-ray binaries (LMXBs).

(see, e.g. Giménez-García et al., 2015, for a recent work). The energy of fluorescence emission is affected in a non-trivial way by the charge state of the ion, and thus it is more difficult to probe the physics of the emitting medium with the corresponding lines compared to the emission lines from the $\mathrm{H}$ and He-like ions discussed in Sect.4.2 However, as we discuss below, the fluorescence lines can be used to investigate the distribution of cold matter around the neutron star.

The profile of the Fe K $\alpha$ fluorescent line in SgXBs can usually be well characterized by using a simple Gaussian profile. Although this is known to be a simplistic approach, fits to the X-ray spectra with such a model generally permit us to estimate the intensity, width, centroid energy, and equivalent width (EW) of the line with a reasonable accuracy, as well as to constrain the time variability of these parameters. High resolution spectra, obtained with the gratings instruments on-board Chandra, have shown that in wind-fed $\mathrm{SgXBs}$ the Fe $\mathrm{K} \alpha$ line is a narrow feature (FWHM $<5 \mathrm{~m} \AA$ ) and it is centred on average at $\lambda=1.9387 \pm$ $0.0016 \AA$ (see the left panel of Fig. 15 and Torreión et al. (2010a)). The general finding that the intensity of the $\mathrm{Fe} \mathrm{K} \alpha$ line directly correlates with the intensity of the source confirms that this feature is produced as a consequence of the illumination of the stellar wind material by the X-ray emission from the compact object (see right panel of Fig. 15). This has recently been also reconfirmed through an in-depth analysis of all available XMM-Newton data on SgXBs (Giménez-García et al., 2015).

The size of the fluorescence emitting region in $\mathrm{SgXBs}$ has been estimated for a number of sources using different methods. Observations of eclipsing wind-fed $\mathrm{SgXBs}$ show that the $\mathrm{EW}$ of the $\mathrm{Fe} \mathrm{K} \alpha$ is generally higher when the systems are in eclipse (see the left panel of Fig. 16). This indicates that at least in some cases the reprocessing region is extending beyond the radius of the massive companion. In other cases it was shown that the bulk the $\mathrm{Fe} \mathrm{K} \alpha$ emission comes from the the immediate surroundings of the compact object, and thus that the accretion wake is likely playing a key role in the $\mathrm{Fe} \mathrm{K} \alpha$ line production (see Sect.4.2). This is the case, for example, of Vela X-1 (Watanabe et al., 2006). In IGR J17252-3616, Manousakis and Walter (2011) inferred the existence of a dense cocoon of cold material with a radius of $\sim 10 \mathrm{R}_{\odot}$ around the neutron star by analysing the variation of $\mathrm{Fe} \mathrm{K} \alpha$ during the eclipse. The size of this structure is thus $\sim 0.5$ times smaller than 

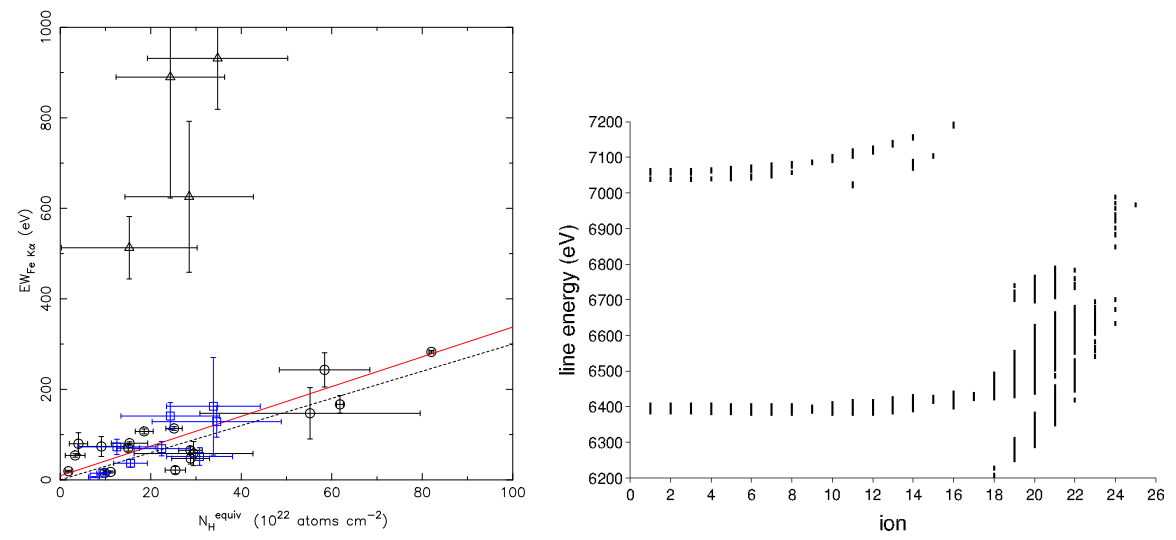

Fig. 16 Left: EW of Fe K $\alpha$ against $N_{\mathrm{H}}$ (plot from Torreión et al., 2010a, reproduced with permission). The EW of the line increases with the column density. Black circles are HMXBs, blue squares are LMXBs, and black triangles are eclipse data. In eclipse the $\mathrm{EW}$ of $\mathrm{Fe} \mathrm{K} \alpha$ is greater because a significant fraction of the continuum is blocked. The black dashed line represents the prediction of the theoretical model in Kallman et al. (2004). Right: in the lower part of the plot the energy of $\mathrm{Fe} \mathrm{K} \alpha$ is shown as a function of the ionization state of $\mathrm{Fe}$ ions (Kallman et al., 2004, reproduced with permission).

the stellar radius estimated for the supergiant hosted in this system (Mason et al., 2009). Audley et al. (2006) used the time delay between the pulsar eclipse and the Fe K $\alpha$ eclipse in OAO $1657-415$ to prove that $80 \%$ of $\mathrm{Fe} \mathrm{K} \alpha$ is emitted in this source within a region of $R<8 \mathrm{R}_{\odot}$. Fürst et al. (2011) analysed the delay in the arrival times of the pulses in the $\mathrm{Fe} \mathrm{K} \alpha$ energy range to conclude that the fluorescence region in GX 301-2 has a radial extension $R<1 \mathrm{R}_{\odot}$. Different results were reported for this source by Endo et al. (2002). The latter authors assumed that the width of the line was caused by the Doppler broadening of the free-fall motion of the accreting matter onto the neutron star and inferred a size of the fluorescence emitting region of $R<0.1 \mathrm{R}_{\odot}$. This is significantly smaller than previous estimates. A plausible reason is that they neglect other broadening mechanisms, such as the blend of multiple fluorescence lines from different ionization states of iron (Fürst et al., 2011). Finally, the alternative method employed by Rodriguez et al. (2006), which takes into account multiple ionization states of iron and the impact of a ionization parameter that changes at different distances from the neutron star, gave an extension of the reprocessing region in the $\mathrm{SgXB}$ IGR J16320-4751 of $R>1.5 \mathrm{R}_{\odot}$.

So far, the observations of wind-fed $\mathrm{SgXBs}$ have been supporting reasonably well the theoretical prediction that the measured equivalent width (EW) of the $\mathrm{Fe} \mathrm{K} \alpha$ line due to $\mathrm{X}$ ray transmission through a optically thin medium is related to the hydrogen column density $N_{\mathrm{H}}$ measured in the direction of a source. The latter is theoretically estimated through Eq. 5 in Kallman et al. (2004):

$$
E W \simeq 300 \mathrm{eV} \frac{N_{\mathrm{H}}}{10^{24} \mathrm{~cm}^{-2}} .
$$

We show in Fig. 16 (left panel) the currently observationally measured correlation between the EW of the Fe K $\alpha$ and the $N_{\mathrm{H}}$ in different SgXBs (see also Giménez-García et al., 2015). In these sources the region reprocessing most of the neutron star X-ray emission is thus also providing a dominant contribution to the material absorbing this radiation along the observer line of sight. 
The centroid energy of the $\mathrm{Fe} \mathrm{K} \alpha$ is one of the measurements of this spectral feature that provides a direct indication of the physical conditions in the wind, as it depends on the ionization state of the Fe ions. In Figure 16 (right panel) we can see that the centroid energy of the line significantly increases with luminosity, especially when the iron is highly ionized (Kallman et al., 2004). Chandra measurements revealed the presence of a number of Fe ions, ranging from Fe II to Fe X (Torreión et al., 2010a). This is compatible with the properties of an O-B supergiant companion, as we expect to find Fe II-III in the wind of a BI star and Fe IV-VI in the wind of an O-star (see, e.g., Kudritzki and Puls, 2000). As can be seen in Fig. 16 (right panel) the centroid energy of the line depends only weakly on the ionization state of the iron ions below $\sim$ Fe XVIII. For the low Fe ionization states, a more sensitive tracer of the conditions in the donor star wind is the ratio between the fluxes of the $\mathrm{Fe} \mathrm{K} \alpha$ and the $\mathrm{Fe} \mathrm{K} \beta$ line (see, e.g. Palmeri et al., 2003). For neutral iron we expect a value of $\mathrm{Fe} \mathrm{K} \beta / \mathrm{Fe} \mathrm{K} \alpha=0.125$ (Kaastra and Mewe, 1993). This ratio increases to $\sim 0.2$ for Fe VIII. However, as generally the flux of the $\mathrm{Fe} \mathrm{K} \beta$ is only $10-20 \%$ of the Fe $\mathrm{K} \alpha$ line, this technique requires data with particularly high statistics in order to perform sufficiently accurate measurements (see, e.g., the case of GX 301-2; Fürst et al., 2011). When calculating the ratio between the fluxes of the $\mathrm{Fe} \mathrm{K} \alpha$ and the $\mathrm{Fe} \mathrm{K} \beta$ line in sources characterized by an absorption column density $>10^{23} \mathrm{~cm}^{-2}$, self-absorption is also expected to play an important role. Theoretically, the effect of self-absorption is different for the two lines and might lead to artificially high evaluated flux ratios. However, it shall be noted that the effect of self-absorption could not be robustly tested yet against high quality observations and thus its anticipated impact on the $\mathrm{Fe} \mathrm{K} \alpha$ and the $\mathrm{Fe} \mathrm{K} \beta$ flux ratio remains to be confirmed.

Another characteristic of the $\mathrm{Fe} \mathrm{K} \alpha$ line that can be used to probe stellar winds is its width in energy. This parameter provides an upper limit on the velocity of the X-ray reprocessing material around the neutron star, as this is generally considered to be the main broadening mechanism for the line (but other mechanisms, such as line blending, cannot be excluded). Chandra observations proved so far that the $\mathrm{Fe} \mathrm{K} \alpha$ line in $\mathrm{SgXBs}$ is relatively narrow (FWHM $<5 \mathrm{~m} \AA$ ), leading to upper limits on the velocity of the X-ray reprocessing material of $\lesssim 1000 \mathrm{~km} / \mathrm{s}$ (Hanke et al., 2009; Torrejón et al., 2010a, 2015). This is in agreement with the typical velocities of $\mathrm{O}-\mathrm{B}$ star winds in $\mathrm{SgXBs}$ but would also be compatible with the free fall velocity of material located at a distance from the neutron star where the Fe $\mathrm{K} \alpha$ line is produced (Endo et al., 2002). The two processes are generally difficult to disentangle, unless accurate measurements of the width of the line are available during different phases of the neutron star orbit (the accretion flow is not expected to be as isotropic as the stellar wind).

In a few $\mathrm{SgXBs}$, the $\mathrm{Fe} \mathrm{K} \alpha$ line has been also observed to show a Compton-shoulder extending down to $\sim 6.2 \mathrm{keV}$. A Compton shoulder forms when X-ray photons propagating through a low temperature medium $\left(\lesssim 10^{5} \mathrm{~K}\right)$ transfer part of their energy to the free electrons via Compton scattering. If the Compton optical depth of the medium is $\gtrsim 0.1$, the probability of those scatterings to occur is not negligible. The resulting effect is more pronounced on emission lines at higher energies, and thus particularly on the $\mathrm{Fe} \mathrm{K} \alpha$ line, due to the energy dependence of the Compton scattering opacity with respect to that of the photoionization. As the shape of the Compton shoulder is sensitive to the electron column density and temperature of the scattering medium, it provides an additional tool to study the physical properties of the cold material around the neutron star. So far, this feature could be clearly detected in the two classical $\mathrm{SgXB}$ s characterized by the highest absorption column densities, i.e., GX 301-2 (Watanabe et al., 2003) and IGR J16318-4848 (Ibarra et al., 2007).

The $\mathrm{Fe} \mathrm{K} \alpha$ line is not the sole fluorescent line that can be detected in the X-ray spectra of SgXB and SFXT sources. As an example, Fürst et al. (2011) reported the detection of 
the $\mathrm{NiK} \alpha, \mathrm{NiK} \beta, \mathrm{CrK} \alpha, \mathrm{CaK} \alpha, \operatorname{ArK} \alpha$, and $\mathrm{SK} \alpha$ fluorescent lines in GX 301-2. Due to the lower fluorescence yield of all these elements compared with iron, these additional spectral features are usually much fainter than the $\mathrm{Fe} \mathrm{K} \alpha$ line and thus it is more difficult to use them as probes of the material around the neutron star.

\subsection{X-ray observations of SgXBs and SFXTs: caveats}

As anticipated in Sect. 4.1 we discuss below the effect of systematic uncertainties affecting two of the key observables obtained from the X-ray data of $\mathrm{SgXBs}$ and used to infer stellar wind parameters: the absorption column density in the direction of a source and its bolometric X-ray luminosity.

\subsubsection{The absorption column density}

The absorption of X-rays in the interstellar medium or close to the X-ray source itself plays a key role in the analysis of X-ray spectra (Wilms et al., 2000; Hanke, 2011). As an observational fact, the flux of any kind of X-ray source located within the Milky Way or beyond is significantly suppressed below $\sim 5 \mathrm{keV}$. This is due to photo-ionization of matter by X-ray photons along the line of sight to the source (see, e.g., Dickey and Lockman, 1990; Mathis, 1990 , for reviews about the interstellar extinction). The effect of absorption on the source X-ray flux $I_{\text {src }}(E)$ at a certain energy $E$ can be described by:

$$
I_{\mathrm{obs}}(E)=I_{\mathrm{src}}(E) e^{-\sigma(E) N_{\mathrm{H}}}
$$

where $I_{\mathrm{obs}}(E)$ is the observed source flux and $\sigma(E)$ is the energy dependent cross-section of the absorbing medium. In $\mathrm{SgXBs}$, the latter comprises the contribution from the interstellar medium (ISM) and from the medium local to the source. The latter is by far the most dominant, and generally the ISM contributes only for a few to $10-30 \%$ (at the very most). However, in those cases in which an accurate estimate of the absorption column density is needed, both contributions have to be taken into account.

In most cases, the estimates of the ISM absorption column density in the direction of a source are assumed to coincide with the measurements of the neutral hydrogen column densities obtained from the Galactic surveys carried out at $21 \mathrm{~cm}$ (Kalberla et al., 2005; Dickey and Lockman, 1990). As pointed out by Wilms et al. (2000), an uncertainty behind this assumption is that the contribution of the Galactic $\mathrm{H}_{2}$ molecular clouds to the total $N_{\mathrm{H}}$ is not considered. These clouds are not uniformly distributed in the Milky Way (Shull and Beckwith, 1982) and thus the assumed abundance of $\mathrm{H}_{2}$ relative to neutral hydrogen should be, in principle, carefully evaluated for each source.

Concerning the material absorbing the X-ray emission local to the source, the problem is even more complicated as the abundances of high $Z$-elements (e.g., oxygen and iron which are responsible for most of the absorption in X-rays) have to be known accurately. These abundances might change among different X-ray sources, as they are expected to be tightly related to the specific binary system environment. The latter is known to be heavily affected by the outflowing material from the massive companion, as well as from the supernova explosion that led to the formation of the neutron star. As these processes of chemical enrichment are not known yet with a reasonably good accuracy, it is typically assumed that the surroundings of a $\mathrm{SgXB}$ are characterized by a solar or ISM-like composition. For the latter, updated models are periodically made available, following our progresses in the understanding of the Milky Way chemical composition. 
Table 4 List of photo-ionization cross-sections ${ }^{\mathrm{a}}$ and abundances ${ }^{\mathrm{b}}$ as available in ISIS or XSPEC, as well as absorption models. ISIS and XSPEC are two of the most used fitting tools for accurate X-ray spectral analysis available to date. In the table below, the asterisk identify the default XSPEC configuration (version 12.8.2).

\begin{tabular}{|c|c|c|}
\hline \multicolumn{2}{|c|}{ Cross-sections } & \multirow{2}{*}{ Comment } \\
\hline obcm & Balucinska-Church and McCammon (1992) & \\
\hline \multirow{2}{*}{$\begin{array}{l}\text { bcmc* } \\
\text { vern }\end{array}$} & Balucinska-Church and McCammon (1992) & \multirow[t]{2}{*}{$\mathrm{H}_{2}$-cross-section from Yan et al. (1998) } \\
\hline & Verner et al. (1996) & \\
\hline \multicolumn{2}{|c|}{ Abundance vector } & Comment \\
\hline aneb & Anders and Ebihara (1982) & solar \\
\hline angr* & Anders and Grevesse (1989) & solar \\
\hline feld & Feldman (1992) & solar \\
\hline grsa & Grevesse and Sauval (1998) & solar \\
\hline wilm & Wilms et al. (2000) & ISM \\
\hline lodd & Lodders (2003) & solar \\
\hline aspl & Asplund et al. (2009) & solar \\
\hline \multicolumn{2}{|c|}{ Absorption models } & Contributions \\
\hline \multicolumn{2}{|c|}{ wabs } & $\begin{array}{l}\text { neutral, atomic, thin gas } \\
\text { (abundances fixed to aneb) }\end{array}$ \\
\hline \multirow{4}{*}{$\begin{array}{l}\text { phabs } \\
\text { tbabs }\end{array}$} & & neutral, atomic, thin gas \\
\hline & Wilms et al. (2000) & neutral, atomic, thin gas \\
\hline & & + neutral $\mathrm{H}_{2}$-molecules \\
\hline & & $\begin{array}{l}\text { + spherical, chemical homogeneous dust grains } \\
\text { (cross-sections fixed to vern) }\end{array}$ \\
\hline tbnew $^{\mathrm{c}}$ & Wilms priv.comm. & improved version of tbabs including high \\
\hline \multirow{3}{*}{$\begin{array}{l}\text { cabs } \\
\text { ctbabs }\end{array}$} & & $\begin{array}{l}\text { resolution cross-sections at important edges } \\
\text { optically-thin Compton scattering }\end{array}$ \\
\hline & Eikmann et al. $(2012,2014)$ & full Compton scattering \\
\hline & & + fluoresence line emission \\
\hline \multirow{2}{*}{\multicolumn{2}{|c|}{ warmabs }} & uniform, collisional ionization (warm stellar winds) \\
\hline & & based on a fixed, ionization-balanced, thin gas \\
\hline \multicolumn{3}{|c|}{ http://heasarc.nasa.gov/xanadu/xspec/xspec11/manual/node36.html } \\
\hline \multicolumn{3}{|c|}{ https://heasarc.gsfc.nasa.gov/xanadu/xspec/manual/XSabund.html } \\
\hline \multicolumn{3}{|c|}{ http://pulsar.sternwarte.uni-erlangen.de/wilms/research/tbabs } \\
\hline
\end{tabular}

Measurements of the absorption column density can thus be derived from the assumed abundances by using the cross-sections and energies for the X-ray absorption on the shell electrons of each of the involved elements. In most of the X-ray sources, the effect of Thomson scattering has also to be taken into account when computing the total X-ray absorption, as part of the material close to the compact object could be significantly ionized and contribute differently to enhance the $N_{\mathrm{H}}$ compared to the neutral material (see Sect. 4.2). At present, not all cross-sections and energies for the Thomson scattering can be derived precisely from the atomic physics and thus most of the absorption models have to rely on specific cross-section tables. Furthermore, when the density of the ionized absorbing material reaches values of $\gtrsim 10^{24} \mathrm{~cm}^{-2}$, Compton-scattering effects also come into play and have to be included in the estimate of the total $N_{\mathrm{H}}$ (Eikmann et al., 2012). An overview of all the currently available absorption models, abundances, and cross sections for the different processes is presented in Table 4 together with their identification code in the two most used software packages for the fitting of X-ray data (i.e., ISIS 30 and XSPE 31 ).

As we can see from Table 4 a number of different absorption models have been published in the literature, each using different assumptions for the ISM cross-sections and abundances of the different elements. To illustrate how the value of the absorption column density in the direction of a source changes when different models are used, we show in Fig. 17 (left panel) the ratio of the two absorption models phabs and tbnew as a function of the energy for $N_{\mathrm{H}}=1 \times 10^{22} \mathrm{~cm}^{-2}$. The main differences between the two models can be

$30 \mathrm{http} / / /$ space.mit.edu/asc/isis/.

31 https://heasarc.gsfc.nasa.gov/xanadu/xspec/. 

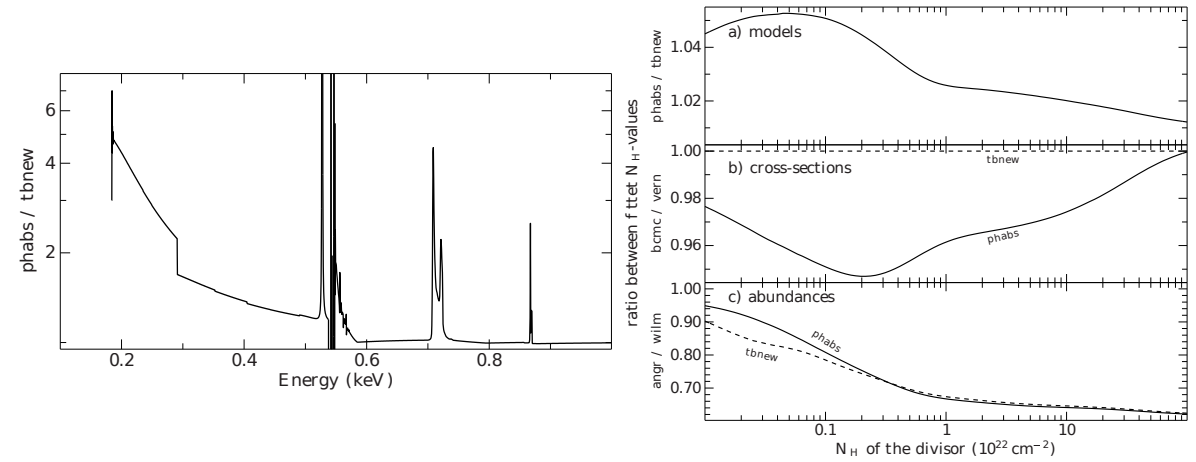

Fig. 17 Left: ratio between the phabs- and the tbnew-absorption-model. An $N_{\mathrm{H}}$ of $1 \times 10^{22} \mathrm{~cm}^{-2}$ was used to calculate the absorbed spectra featuring a constant photon flux. The cross-sections were set to vern and the abundances to wilm. Right: comparison of derived $N_{\mathrm{H}}$-values from X-ray spectra based on a) different models, b) cross-sections, and c) abundances. The assumed $N_{\mathrm{H}}$ using a specific configuration is plotted against the $N_{\mathrm{H}}$-ratio when fitted with a different configuration. The cross-sections and abundances for a) were set to vern and wilm, respectively. In b) and c), the results using either the phabs- (solid line) or the tbnewabsorption-model (dashed line) are also shown. References to the photo-ionization cross-sections, abundances and absorption models given in Table 4

noted around the energies corresponding to the $\mathrm{K}$-edges of oxygen $(0.53-0.58 \mathrm{keV})$, neon $(0.87 \mathrm{keV})$, and the L-edge of $\mathrm{Fe}(0.71 \mathrm{keV}-0.73 \mathrm{keV})$. Furthermore, phabs absorbs the $\mathrm{X}$ ray spectrum less than tbnew for energies below $0.6 \mathrm{keV}$ (up to a factor of $\sim 5$ at $0.2 \mathrm{keV}$ ), and thus the $N_{\mathrm{H}}$ values derived with phabs are systematically higher than those derived with tbnew. This is illustrated in the right panel of Fig. 17, where several spectra were simulated over a wide range of $N_{\mathrm{H}}$ values by using tbnew and fit with phabs. The largest discrepancy between the two models is about $5 \%$ at $5 \times 10^{20} \mathrm{~cm}^{-2}$. Similarly, the derived value of the $N_{\mathrm{H}}$ depends on the cross-sections and abundances used. Panel b) and c) on the right side of Fig. 17] show these effects as a function of $N_{\mathrm{H}}$. Outdated cross-sections, as bcmc, underestimate the column density up to $5 \%$ (note that the cross-sections in tbnew are fixed to vern). The most dramatic differences in values of the inferred $N_{\mathrm{H}}$ are obtained when models adopting solar and ISM-abundances are compared: in the first case, values of the absorption column density exceeding $10^{21} \mathrm{~cm}^{-2}$ are all underestimated by more than $20 \%$. This effect is more pronounced for higher values of $N_{\mathrm{H}}$.

From the above results, it is clear that any measurement of the absorption column density in a X-ray source, and particularly in $\mathrm{SgXBs}$, is significantly dependent on the chosen absorption model, cross-section, and abundances prescription. In case $N_{\mathrm{H}}$ values derived from the X-ray observations are used to infer the physical properties (e.g., density, mass, and size) of structures in the stellar wind around a neutron star, it would be a good practice to clearly state the models and prescriptions used. In this way, possible discrepancies between different findings can be investigated in terms of systematic uncertainties among the different absorption models or ascribed to intrinsic changes in the properties of the absorbing medium local to the source.

\subsubsection{The neutron star bolometric X-ray luminosity}

The intrinsic bolometric X-ray luminosity $L_{X}$ of an $\operatorname{SgXB}$, that as we saw in the previous sections is a key parameter to infer the stellar wind parameters and the details of the accretion 


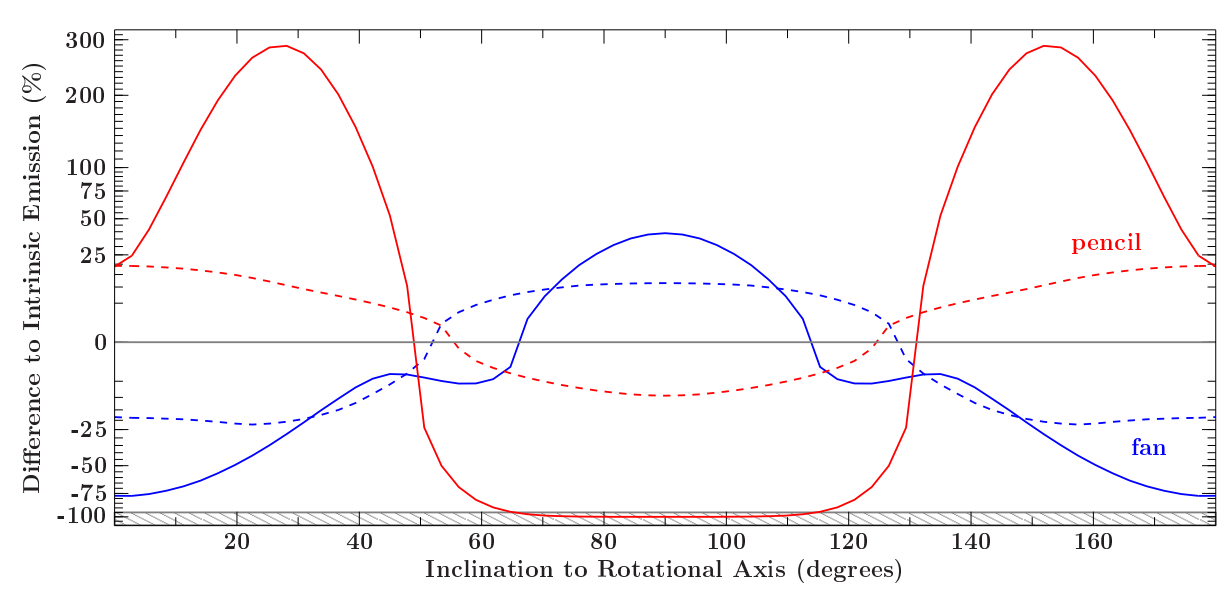

Fig. 18 Relative difference of the derived luminosity to the real intrinsic luminosity depending on the inclination to the neutrons star's rotational axis. The derived luminosity is calculated from the observed flux assuming isotropic emission ( $4 \pi$-factor). The intrinsic luminosity assuming a Gaussian (solid lines) or no beam pattern (dashed lines) is calculated for a pencil beam (blue) and a fan beam geometry (red). The dashed area corresponds to observed fluxes $\leq 5 \%$ off the intrinsic flux, which would result in an apparently dim pulsar.

processes, is commonly derived from the measured X-ray flux $F_{X}$ of the source by using the relation:

$$
L_{\mathrm{X}}=4 \pi d^{2} F_{\mathrm{X}}
$$

Here, it is assumed with the $4 \pi$ factor that the neutron star's X-ray radiation is emitted isotropically. In most of the cases of interest for this review, the larger source of uncertainty on $L_{\mathrm{X}}$ is due to the poorly known distance to the system, $d$. As it can be seen in Table 2, this distance is often known only within an accuracy of a factor $\sim 2-3$ and for some of the $\mathrm{SgXBs}$ contradicting measurements have been reported.

The rough assumption about the isotropic nature of the X-ray emission introduces in all cases an additional source of uncertainty. This assumption does not generally apply to accreting neutron stars: the bulk of the X-rays are produced in the accretion columns near the two magnetic poles, where accreting matter hits the neutron star surface (see Becker and Wolff, 2007, and references therein). Furthermore, the emission profiles of the accretion column (or the hot spots on the surface) are not known and might be a complex combination of a fanor pencil-beam (see, e.g., Harding, 1994).

The emission patterns are also distorted by general relativistic effects in the strong gravitational field of the neutron star. In most cases, both magnetic poles are visible over one full rotation of the neutron star (Beloborodov, 2002). Depending on the geometry and the position of the observer, the total flux might be beamed and boosted towards the observer, or pointed in a different direction and lowered in intensity. To provide an estimate of this effect we have used an extended light-bending code, which is based on work by Kraus (2001). The new code, allowing for more complicated accretion column and emission geometries, has been used already to explain phase-shifts at the cyclotron line energies seen in the pulseprofiles of some accreting neutron stars (Schönherr et al., 2014). We have assumed standard neutron star parameters for the mass and radius, and a magnetic axis going through the star's center inclined by $30^{\circ}$ with respect to the rotational axis. In Fig. 18 we show, as a function of the inclination angle to the observer, the difference between the computed emission and 
the real intrinsic emission in the case of: (i) a pencil beam with two hotspots of $1 \mathrm{~km}$ radius located at the neutron star magnetic poles, (ii) a fan beam with two accretion columns of $1 \mathrm{~km}$ in radius and $2 \mathrm{~km}$ in height located at the neutron star magnetic poles. In both cases we have simulated a non-beamed emission from the emission regions and a Gaussian beam pattern with a half opening angle of $10^{\circ}$ (see, e.g., Basko and Sunyaev, 1975). This beam pattern accounts for the angular distribution of the emerging photons, which boosts the photons leaving (i) the hot spots perpendicular to the surface in case of a pencil-beam and (ii) the accretion columns sideways in the fan-beam case. The calculated difference in Figure 18 can be considered as the error that would be introduced on the estimate of the source luminosity by assuming a simplified isotropic emission pattern instead of the correct one. The introduced error is larger in the pencil beam geometry, where the beamed-case easily leads to an over-estimation as large as $\sim 300 \%$. There is a distinct range of inclinations between $50^{\circ}$ and $130^{\circ}$ where the derived luminosity is drastically under-estimated. The pulsar is even not detectable between $70^{\circ}$ and $110^{\circ}$ since the pencil beam never "strikes" the observer (dashed region in Fig. 18). In the fan beam geometry the effect is more modest, with the largest recorded over-estimation reaching $\sim 40 \%$. At inclinations between $-20^{\circ}$ and $+20^{\circ}$ the luminosity is underestimated, however, by $-40 \%$ down to $-75 \%$. For both considered emission cases, the error on the estimated luminosity is limited to a maximum of $\pm 25 \%$ once no beam pattern is assumed (dashed lines in Fig. 18), which can be interpreted as a lower limit. We stress that, however, all these estimated differences strongly depend on the assumed emission geometry, like the location of the magnetic poles. The differences can become even larger if: (i) a less favourable geometry is considered for the inclination of the neutron star's magnetic axis with respect to its rotational axis and for the emission pattern; (ii) more extreme neutron star spin periods and magnetic fields are explored.

Finally, a last source of uncertainty comes from the source X-ray flux obtained form the observational data. Beside uncertainties due to the calibrations of the X-ray instruments used to carry out the observations (Guver et al.,2015, see, e.g.,), the bolometric flux of a source is usually measured on a relatively small energy band (e.g. $0.5-10 \mathrm{keV}$ ) and then extrapolated to a much wider range to obtain the bolometric luminosity (typically $0.1-100 \mathrm{keV}$ ). This procedure can lead to a significant over- or under-estimation of the source bolometric flux, especially for those cases in which the spectral energy distribution is not accurately known and fits to the observed X-ray spectra are performed with phenomenological rather than physically justified models (see the discussions in, e.g., Becker et al., 2012; Farinelli et al., 2012a, b).

It thus seems that, whenever accurate measurements are required to derive the physical parameters of stellar winds or details on the accretion processes in $\mathrm{SgXBs}$ from an X-ray observation, caution should be taken on the estimated values of the source luminosity.

\subsection{The peculiar case of the SFXTs}

SFXTs and classical $\mathrm{SgXBs}$ are known to have optical counterparts with similar properties (Masetti et al., 2006; Chaty et al., 2008; Rahoui et al., 2008), even though dedicated opti$\mathrm{cal} / \mathrm{IR} / \mathrm{UV}$ spectroscopic monitoring campaigns of all these sources have been performed only rarely (especially for the SFXTs; see, e.g. Lorenzo et al., 2010, 2014). In all cases, these campaigns could only provide limited information on stellar winds, as all $\mathrm{SgXBs}$ are located several kpc away and the high extinction toward their directions makes it impossible to carry out detailed spectroscopic investigations as it is done for close-by supergiants (see Sect. 3. 
As we discussed in Sect. 4.1, if one assumes that the flares in SFXTs are produced by direct accretion (see Sect. 2.2.2) of wind clumps onto the neutron star (as originally suggested by in't Zand, 2005), the derived clump masses can be as large as $10^{23} \mathrm{~g}$ and the required density ratio between the clumps and the intra-clumps material should be of the order of $10^{4}-10^{5}$ (see Sect. 4.1 and Walter and Zurita Heras, 2007; Levder et al., 2007; Ducci et al., 2009, 2010; Romano et al., 2010, 2012; Bozzo et al., 2011). These values are far too extreme compared to the more moderate clumpy winds that are expected according to the results of the latest observational campaigns and numerical simulations of hot stellar winds (see Sect.4.1). Therefore, it seems that a simple clumpy wind scenario is not able to account for the variability of the SFXTs, at odds with what has been found for the classical SgXBs in Sect. 4.1. Other mechanisms have thus to be invoked.

The similarity in the orbital periods of most SFXTs and classical SgXBs complicates the picture even more, so that models comprising only the accretion from the clumpy supergiant wind and peculiar orbital geometries (Negueruela et al., 2006a) are not able to fully explain the difference between the two classes of systems. However, the high proper motion recently measured in the prototypical SFXT IGR J17544-2619 supports the suggestion that at least some SFXTs might have highly eccentric orbits (Maccarone et al., 2014). This would lead to a slight decrease of the averaged X-ray luminosity and an increase in the achievable dynamic range of the system, as a neutron star on an eccentric orbit spends a long time away from its companion in regions where the stellar wind (and thus the accretion) is lower (Negueruela et al., 2006a). With the increasing observing time targeted on SFXTs in the last 10 years, it became eviden 32 that the case of IGR J17544-2619 might not be unique and it was found that bright flares in a number of SFXTs appear clustered at certain orbital phases, assumed to be the periastron passage of a somewhat elongated orbit (Drave et al., 2010; Romano et al., 2014b). However, even in these cases, flares do not necessarily occur at every periastron passage (Sguera et al., 2007; Drave et al., 2014) and intense periods of Xray activity have been recorded by the same sources at larger distances from the supergiant (see, e.g., Goossens et al., 2013) or spread along the entire orbit (Smith et al., 2012). Controversial results about the distribution of flares along the orbit of XTE J1739-302 have been obtained by different teams, although probably due only to different instrumental sensitivity and observing strategy (Smith et al., 2012; Drave et al., 2010).

The long term monitoring observations of many SFXTs, that were carried out mainly to search for the outbursts of these sources and the most likely orbital phases at which these events are occurring, clearly evidenced that these systems are significantly sub-luminous compared to other classical SgXBs. In particular, their average X-ray luminosity was measured to be a factor of 100 lower than that expected from a classical SgXB with a similar orbital period (Romano et al., 2011, 2014a). In IGR J16479-4514, the SFXT with the shortest orbital period ( $\sim 3$ days) and, very likely, a circular orbit, a Suzaku observation allowed to almost continuously and deeply cover $80 \%$ of the orbit at X-rays (Sidoli et al., 2013). In this source, the ratio between the scattered X-rays observed during the $\mathrm{X}$-ray eclipse and the level of the uneclipsed emission permitted to derive an estimate of the wind density at the neutron star distance of $7 \times 10^{-14} \mathrm{~g} \mathrm{~cm}^{-3}$ (Sidoli et al., 2013). Assuming a spherical outflowing supergiant wind, this density implies a ratio between the wind mass loss rate and the wind terminal velocity $\dot{M}_{w} / v_{\infty}=7 \times 10^{-17} \mathrm{M}_{\odot} / \mathrm{km}$. Assuming terminal wind velocities from 500 to $3000 \mathrm{~km} \mathrm{~s}^{-1}$ (see Sect. 2.1), this ratio would imply an X-ray luminosity of

\footnotetext{
32 We are not considering here in detail the case of the peculiar SFXT IGR J11215-5952, where the outbursts are strictly periodic (Sidoli et al., 2006, 2007) and they occur at each periastron passage (Romano et al., 2009a).
} 
$\mathrm{L}_{\mathrm{X}}=3-15 \times 10^{36} \mathrm{erg} \mathrm{s}^{-1}$ according to the standard wind accretion scenario (see Eq. 22). This is more than two orders of magnitude higher than the observed luminosity. The conclusion of all these studies is thus that, besides clumps and orbital elongations (only in some cases), a mechanism should be at work in all SFXTs to substantially reduce the mass accretion rate and the average X-ray luminosity.

A first attempt to address these issues by combining wind accretion models with hydrodynamic simulations of massive star winds was carried out by Oskinova et al. (2012), who derived the implied X-ray variability of the system by assuming a direct accretion scenario and circular orbit 33 (see also Sect.4.1). They obtained an extreme and continuous X-ray variability at any orbital phase of the system achieving up to eight orders of magnitude in luminosity. Not only this variability is significantly larger than that observed in the SFXTs, but also the sub-luminosity issue of these sources could not be addressed. On one hand, the large variability is related to the fact that the authors employed 1D hydrodynamic simulations of the stellar wind, in which clumps are known to grow to large sizes and densities (i.e., much larger than those expected from multi-dimensional simulations, see Sect. 2.1.2). On the other hand, this work highlighted again the need for an additional mechanism to reduce the average mass accretion rate onto the neutron star, independently from the properties of the stellar wind.

As illustrated in Sect.2.2.2, a viable solution to reduce the mass accretion rate onto the neutron star is to assume that either centrifugal/magnetic gates are at work, or a settling accretion regime sets in. The possibility of having a centrifugal inhibition of accretion in SFXTs was first proposed by Grebenev and Sunyaev (2007). If the neutron stars in SFXTs are spending most of their time in the supersonic propeller regime, a reduction of the $\mathrm{X}$ ray luminosity by a factor of 100-1000 can be achieved compared to the standard direct accretion regime by assuming the neutron star is endowed with a standard magnetic field $\left(\mu_{30} \simeq 0.1-1\right)$ and a spin period in the range $\simeq 10-100 \mathrm{~s}$ (considering values for the stellar wind parameters in agreement with those expected for O-B supergiants, see Eq. 26. and 35. Combining the centrifugal barrier with the presence of a moderately clumpy wind, one could argue that the sporadic encounter of a dense clump with the neutron star could provide the required increase in the local density to switch from the supersonic propeller to the direct accretion regime (see Eq. 45) and produce a bright short flare as observed in the SFXTs. If a reasonable spread in density between different clumps is assumed, the X-ray dynamic range of the supersonic propeller could be combined with that implied by the simple direct accretion from a clumpy wind (a factor $\sim 10-100$, see Sect.4.1) to nearly explain the extreme variability of the SFXTs. The problem with the sole adoption of the centrifugal gate and the clumpy wind is that such combination of mechanisms would not be able to explain why only the SFXTs and not the classical SgXBs achieve an extreme X-ray variability: the neutron stars hosted in these systems are characterized by similar spin periods and in some cases these can be significantly longer than 10-100 s (Table2).

An alternative possibility would be to combine the presence of a magnetic gate with a clumpy wind model. As it was shown in Sect. 2.2.2 the magnetic gate can easily lead to variations in the system luminosity by a factor of $10^{5}$ and thus it would be better suited to explain the SFXT X-ray dynamic range (see Eq. 28, 29, and 22). The advantage of the magnetic gate, compared to the centrifugal gate, is that it would require extremely large neutron star magnetic fields to be at work $\left(\mu_{30} \simeq 10-100\right)$ and particularly long spin periods

\footnotetext{
33 In their calculations the neutron star is not properly orbiting the companion but is assumed to be fixed at a certain distance while the variable and evolving wind is blown in its direction. The feedback of the X-ray radiation onto the stellar wind is also not included.
} 
( $>1000$ s). These values might not be unreasonable for a young neutron star in an HMXB and could provide a way to differentiate SFXTs from classical SgXBs (Bozzo et al., 2008). According to this idea, SFXTs should host neutron stars with systematically longer spin periods and more intense magnetic fields than classical SgXBs. However, to date, there are no consolidate measurements of SFXT spin periods (see Table2), and the only SFXTs where a hint of the neutron star magnetic field value has been derived, shows a low field of a few $10^{11} \mathrm{C}^{34}$ (Sguera et al., 2010) or a few $10^{12} \mathrm{G}$ (Bhalerao et al., 2015).

A different way to significantly reduce the accretion rate onto the neutron star with respect to the direct accretion regime is the onset of a settling accretion regime. As we discussed in Sect. 2.2.2, this regime is believed to set in when the accreting material is not efficiently cooled down at the accretion radius (Eq. 14) and the Rayleigh-Taylor instability is only able to drive a reduced mass flow rate through the neutron star magnetosphere. Depending on the dominating cooling mechanism at the magnetospheric radius, it can be seen from Eq. 41 and 42 that the accretion rate can be reduced by a factor ranging from 3 to 10 with respect to the direct accretion regime. On one hand, these reductions in the mass accretion rates are too small to reproduce the entire dynamic range of the SFXTs, and in particular to explain their lowest luminosity states $\left(L_{X} \simeq 10^{32} \mathrm{erg} \mathrm{s}^{-1}\right)$. As both Eq. 41 and 42 give the reduction in mass accretion rate as a function of the average source luminosity, it would be possible to achieve the low X-ray emission level of the SFXTs in quiescence within the settling accretion regime only by assuming that SFXTs are a priori significantly fainter in X-rays than the classical SgXBs. This can only be realized if the average mass-loss rate of the supergiant stars in the SFXTs is lower by a factor of 10-100 compared to those of supergiant stars in classical SgXBs. This reduction in mass-loss rate is, however, difficult to reconcile with the present information we have available on these stars, as the latter do not seem to be systematically different from supergiants in classical $\mathrm{SgXBs}$ (see Sect. 4). On the other hand, the high X-ray luminosity reached during the SFXTs brighter outbursts is not easily achieved within the settling accretion regime and would require the presence of very dense and massive clumps to increase the local mass accretion rates onto the neutron star by a factor of $\sim 100-1000$. As such large clumps seem not realistic given our present knowledge of massive star winds (see Sects.2.1.2, 3.9 and 4.1, but also 2.1.3, an alternative possibility was discussed by Shakura et al. (2014). These authors suggested that the bright SFXT outbursts could be triggered by the collapse onto the neutron star of all (or part of) the hot shell that forms around the compact object in the settling accretion regime. The collapse of the shell might occur sporadically due to reconnection events between the supergiant magnetic field embedded in the accreting material and the neutron star magnetosphere 35 . The advantage of this idea is that the excess mass accretion rate required to reach the luminosities of the SFXTs during their outbursts is provided by accumulation within the hot shell around the neutron star rather than the presence of large clumps. Shakura et al. (2014) noted that the typical energy released in an SFXT bright flare $\left(10^{38}-10^{40} \mathrm{erg}\right)$ would be consistent with the estimated mass of the entire hot shell around the compact object (see also the discussion in Drave et al., 2014).

Paizis and Sidoli (2014) also proposed that the settling accretion model seems to be supported by the hard X-ray (20-100 keV) cumulative luminosity distributions of the SFXTs bright flares as observed with INTEGRAL (IBIS/ISGRI) in the past 10 years. The main observational result of this study is that the cumulative luminosity distributions of the SFXTs

\footnotetext{
34 Note that this measurement has not been confirmed by Sguera et al. (2015).

35 Note that this event is considered sporadic because it requires the two magnetic fields to be aligned along a preferred geometry and it is being assumed here that only the winds of supergiant stars in SFXTs are sufficiently magnetized to give rise to such effects as bright outbursts are not observed in classical $\mathrm{SgXBs}$.
} 
flares are well described by a power-law and seem different from the one shown by classical persistent HMXBs (Vela X-1 and 4U 1700-37, where the most frequent state is at high luminosity, around $\left.1-2 \times 10^{36}\right)$. The latter are more similar to log-normal distributions, as expected for a system accreting from a clumpy rather than from a smooth wind (see Sect. 4.1). Paizis and Sidoli (2014) noted that the power-law fit of the SFXT cumulative luminosity distributions is reminiscent of Self-Organized Criticality (SOC) Systems (see, e.g., Aschwanden, 2013). A SOC (Bak et al., 1987) is a system naturally evolving into a critical state where a minor event is able to start a chain reaction leading to a catastrophe, like the unpredictable "avalanches" in a sand-pile, when a certain instability threshold is reached. Several phenomena are believed to behave like SOC systems: solar flares, lunar craters, earthquakes, landslides, forest fires, ...(Newman, 2005). The power-law scaling of SFXTs cumulative luminosity distributions was thus considered suggestive of the fact that SFXTs flares can be associated to "avalanches" in SOC systems, which are triggered when a critical state is reached. This would provide support in favour of the settling accretion model, where the hot matter stored in the shell can penetrate the magnetosphere only if it is able to cool down to a critical temperature (see discussion above and Sect. 2.2.2). According to this interpretation, the observed power-law luminosity distributions of SFXTs flares might be coupled to the properties of the magnetized stellar wind and the physics of its interaction with the neutron star magnetosphere (Shakura et al., 2014), in analogy to what happens, e.g., in the case of the solar flares (these are believed to be produced by the reconnection of the coronal magnetic field and show a power-law distribution; Datlowe et al., 1974).

The results presented by Paizis and Sidoli (2014) were later criticized by Bozzo et al. (2015). These authors used the long-term monitoring observations of many SFXTs carried out with the XRT on-board Swift to build the cumulative luminosity distributions of the SFXTs emission in the soft X-ray domain (0.5-10 keV). Thanks to the higher sensitivity of the XRT compared to ISGRI for the low luminosity states of the SFXTs, it was possible to show that the approximated power-law shape of the cumulative luminosity distributions of these sources is no longer valid when the investigated emission range extends all the way down to $\simeq 10^{33} \mathrm{erg} \mathrm{s}^{-1}$. From these results, it was concluded that the Swift/XRT cumulative luminosity distributions of the SFXTs could have a similar functional shape as those of other $\mathrm{SgXBs}$ in the soft X-rays, but they are simply shifted to lower luminosities. This shift was interpreted as additional evidence of the strong inhibition of accretion that seems to affect all SFXTs (see also Romano et al., 2014a). We note that the discrepancy between the soft and hard X-ray cumulative luminosity distributions of the SFXTs could be explained by the fact that INTEGRAL caught only the emission during the flares (so that the cumulative luminosity distributions reported by Paizis and Sidoli (2014) are distributions of the X-ray flares peaks only), while the luminosity distributions reported by Bozzo et al. (2015) include data collected from all SFXT emission states. At present it is not possible to discriminate through the XRT data if the inhibition of accretion in SFXTs (compared to other SgXBs) is due to the presence of a centrifugal/magnetic gate or the onset of a settling accretion regime. As discussed by Bozzo et al. (2015) neither of the two possibilities seem able at present to satisfactorily explain the difference between classical SgXBs and SFXTs, as one would require large spin periods and magnetic fields that are not measured in the SFXTs and the other would imply a substantially lower mass-loss rate from the supergiant stars in SFXTs for which there is no clear supporting evidence. The issue of the peculiar SFXT $\mathrm{X}$-ray variability thus remains highly debated.

Finally, it cannot be excluded that the class of SFXTs is inhomogeneous, including massive binaries where different mechanisms driving the X-ray flaring transient behaviour are at work: sources where the flaring activity is sporadic and unpredictable are probably dif- 
ferent from SFXTs where the outbursts are periodic, as it occurs for IGR J11215-5952 (Sidoli et al., 2006, 2007). For this source, Sidoli et al. (2007) proposed that the periodic and short outbursts are produced when the neutron star crosses a denser and slower component in the supergiant wind, inclined with respect to the orbital plane. This putative second component of the supergiant wind could probably be due to the donor magnetic field that is able to steadily compress the out-flowing wind onto the magnetic equatorial plane. The minimal supergiant magnetic field, $\mathrm{B}_{\min }$, needed to compress the wind can be estimated from the "wind magnetic confinement parameter" $\eta$ (ud-Doula and Owocki, 2002), assuming $\eta=1: \mathrm{B}_{\min }=\sqrt{\dot{M}_{\mathrm{w}} v_{\infty}} / R_{*}$. Following Romano et al. (2009b) and assuming the updated stellar parameters reported by Lorenzo et al. (2014), $\left(\mathrm{R}_{*}=40 \mathrm{R}_{\odot}, \dot{M}_{\mathrm{w}}=10^{-6} \mathrm{M}_{\odot} \mathrm{yr}^{-1}\right.$, $v_{\infty}=1200 \mathrm{~km} \mathrm{~s}^{-1}$ ), one would get $B_{\min } \sim 30 \mathrm{G}$. So far, a measurement of the magnetic field in the supergiant companions of SFXTs has not been performed. If doable, it could help investigating the applicability of the proposed model for IGR J11215-5952 in the near future. It should be noted, however, that no evidence for the second dense wind component around the supergiant star in IGR J11215-5952 has been found to support the model proposed by Sidoli et al. (2007), despite the intensive searches carried out by Negueruela et al. (2010).

A slightly different interpretation of the peculiar SFXT IGR J11215-5952 was proposed by Lorenzo et al. (2014). These authors obtained high-resolution spectra of the optical counterpart of IGR J11215-5952, HD 306414, spanning three months and including one of its outbursts (in 2007 February). The supergiant star HD 306414 showed significant variability of the $\mathrm{H}_{\alpha}$ line (shape and centroid of the emission feature) simultaneously with the X-ray outburst, but similar variabilities were also observed seven weeks before the peak of the X-ray outburst (i.e. about $1 / 3$ of the orbital period) when no X-ray flaring activity was expected. It was thus suggested that IGR J11215-5952 might have a large eccentricity (around 0.8 ) and that the neutron star in this system is able to accrete material from the wind of the companion only when it is close to its atmosphere during the periastron passage. In this configuration, the accretion would thus most likely take place from a transient tidal stream through the inner Lagrangian point, which could lead to the formation of a transient accretion disk, in turns triggering the periodic X-ray outbursts.

The formation of short-lived accretion disks around the neutron stars in the SFXTs is also a possibility that has been discussed in several works to enhance the achievable X-ray luminosity in these systems. So far, no observational evidence of an accretion disk could be found during the observations of quiescent and out-bursting SFXTs, but Ducci et al. (2010) showed that an accretion disk could be formed especially in systems characterized by a short orbital period, a high X-ray luminosity, and a non negligible eccentricity. The idea is that in those cases in which the X-ray luminosity is high enough to significantly slow down the stellar wind at the neutron star location and the compact object is close to the supergiant, the accretion radius becomes large enough that the angular momentum of the accreting material can not be neglected any longer. Although the formation and dissipation of these short-lived accretion disks is far from being understood, it is well known that an accretion disk can sustain a significantly higher mass accretion rate than the one of a windfed system and can thus lead to higher X-ray luminosities (see, e.g., Frank et al., 2002). At least in one case, it has been convincingly shown that the peak luminosity reached during an SFXT outburst was far too high to be achievable through accretion from a supergiant stellar wind and it was suggested that a disk around the neutron star was formed slightly before the event (Romano et al., 2015). However, no significant changes in the X-ray emission from the source or other observational features could be found to firmly support this idea. Another extreme behaviour from a SFXT has been observed by INTEGRAL in the SFXT IGR J18483-0311 (Sguera et al., 2015), with an unusually long outburst spanning 11 days 
(instead of a few hours), an interval of time lasting about $60 \%$ of the orbit. The long-based observational data-set available now, after 10 years since the SFXTs' discovery, is allowing us to catch even the most extreme (and rare) behaviours from the members of the class. 


\section{Conclusions}

This review attempts to combine the efforts of two different scientific communities that have been independently working to improve our understanding of massive star winds by using either isolated objects or massive stars in binary systems. We have largely reviewed the stateof-the-art of the research in the two fields and showed that the driving aim of these studies in both communities has been the investigation of the physical properties of dense structures populating the surroundings in isolated massive stars and feeding the compact objects in massive binaries. In the former, our relatively limited knowledge on the properties of these structures lead to systematic uncertainties in the estimated mass loss rates from massive stars. Consequently, our ability to reconstruct the long term evolution of these sources and to properly evaluate their role influencing their environment is limited. In the field of massive binaries, in turn, the lack of a proper understanding of the same structures represents one of our major limitations in the theoretical modelling of the wind accretion process onto the compact objects and thus on the ability to predict and reproduce their extreme variability in the X-ray domain (where the bulk of the energy from these systems is released).

The investigation of stellar winds in isolated massive stars has progressed significantly in the past years not only thanks to the availability of improved high sensitivity data in the optical, UV, and X-ray domain, but also to the development of more and more sophisticated numerical simulations. Although many advances have been made in different areas, we illustrated in the course of this review how a large number of fundamental issues are still far from being solved. Among these, various approaches are used by different groups in applying micro and macro-clumping methods, sometimes resulting in different derived mass loss rates and wind properties of massive stars. The formation and origin of wind clumps is also still a matter of debate, as different mechanisms have been proposed to produce these structures and drive their evolution in shape, density, and velocity over time.

In massive binaries, we particularly highlighted in the review how the degeneracy between the properties of the accretion flow regulated by the stellar wind (wind velocity and density, mass loss rates, ionization status) and the physical parameters of the compact object (mainly mass, radius, spin, and magnetic field strength) in all available models makes it very challenging to understand the details of the accretion processes and distinguish among the different proposed mechanisms to drive the release of the high energy radiations from these systems. The advent of the current generation of X-ray observatories, the main energy domain where observations of these systems are carried out, permitted to gain important insights into the accretion of stellar winds onto compact objects. This has been possible mainly through spectral and timing analyses performed by using integration times as short as the dynamical timescales of the relevant physical processes (heating/cooling of the accretion flow, free-fall motion). These results opened the interesting possibility of using the compact objects in these systems as probes in situ of the stellar wind and its structures. This could thus provide, in principle, independent measurements to be compared with those derived from the studies of isolated massive stars. The development of detailed theoretical models and advanced numerical simulations of wind accreting systems has shown, however, that it is very challenging to distinguish between different possible accretion regimes. The gravitational field of the compact object, as well as its rotational period and magnetic field strength, can greatly alter the geometry of the accretion flow. Thus the properties of the stellar wind and its structures inferred from measurements of the X-ray luminosity and spectral variations in the binary sources have to be taken with caution. The availability of more predictable properties for the stellar wind and its structures for a given massive star 
with a known spectral classification could allow us to remove some of the degeneracies and distinguish between different proposed accretion scenarios.

It thus seems that combining observational, theoretical, and simulation efforts of massive star winds and wind accretion in binary sources could certainly lead to significant simultaneous advantages for both fields of research, advancing our understanding of the accretion physics as well as of the galactic and cosmic evolution. For this reason we intend to pursue the common interests of this combined community in the years to come by promoting: (i) the development of a new generation of hydrodynamic/magnetohydrodynamic simulations that can take into account the formation and evolution of stellar winds in presence of a compact object; (ii) the organization of multi-wavelength observational campaigns that take mutual advantage of the different diagnostic techniques exploited for isolated massive stars in the optical/UV/X-rays with those adopted by the binary community to the properties of stellar winds; (iii) advances in theoretical studies of the interaction between the X-ray radiation from accreting compact objects and stellar winds, especially in cases of highly inhomogeneous flows.

\section{Acknowledgements}

We are grateful to the two anonymous referees for carefully reviewing such a large and detailed piece of work and for providing very useful comments, which have allowed us to improve this paper.

SMN acknowledges the support of the Spanish Unemployment Agency, which allowed her to continue her scientific collaborations while being temporarily unemployed during the critical situation of the Spanish Research System until March 2016.

SMN acknowledges support by research project ESP2016-76683-C3-1-R.

LS acknowledges the Italian Space Agency financial support INTEGRAL ASI/INAF agreement n. 2013-025.R.0, and the grant from PRIN-INAF 2014, "Towards a unified picture of accretion in High Mass X-Ray Binaries" (PI: Sidoli).

AGG acknowledges support by Spanish MICINN under FPI Fellowship BES-2011050874 and the Vicerectorat d'Investigació, Desenvolupament i Innovació de la Universitat d'Alacant under project GRE12-35.

IK, MK, and JW are supported by the Bundesministerium für Wirtschaft und Technologie under grant number 50OR1207 of the Deutsches Zentrum für Luft- und Raumfahrt. MK also acknowledges support by the Bundesministerium für Wirtschaft und Technologie under Deutsches Zentrum für Luft- und Raumfahrt grant 50OR1113.

AS is supported by the Deutsche Forschungsgemeinschaft (DFG) under grant HA 1455/26.

JMT acknowledges research grants ESP2013-48637-C2-2P and ESP2014-53672-C3-3-

P.

This publication was motivated by a team meeting sponsored by the International Space Science Institute at Bern, Switzerland.

\section{References}

D.C. Abbott, The theory of radiatively driven stellar winds. I - A physical interpretation. ApJ 242, 1183-1207 (1980). doi:10.1086/158550

D.C. Abbott, The theory of radiatively driven stellar winds. II - The line acceleration. ApJ 259, 282-301 (1982) 
D.C. Abbott, J.H. Bieging, E. Churchwell, J.P. Cassinelli, VLA radio continuum measurements of mass loss from early-type stars. ApJ 238, 196-202 (1980). doi:10.1086/157973

E. Alecian, G.A. Wade, C. Catala, J.H. Grunhut, J.D. Landstreet, S. Bagnulo, T. Böhm, C.P. Folsom, S. Marsden, I. Waite, A high-resolution spectropolarimetric survey of Herbig Ae/Be stars - I. Observations and measurements. MNRAS 429, 1001-1026 (2013). doi:10.1093/mnras/sts383

E. Anders, M. Ebihara, Solar-system abundances of the elements. Geochim. Cosmochim. Acta 46, 2363-2380 (1982)

E. Anders, N. Grevesse, Abundances of the elements - meteoritic and solar. Geochim. Cosmochim. Acta 53, 197-214 (1989)

A. Ankay, L. Kaper, J.H.J. de Bruijne, J. Dewi, R. Hoogerwerf, G.J. Savonije, The origin of the runaway high-mass X-ray binary HD 153919/4U1700-37. A\&A 370, 170-175 (2001). doi:10.1051/0004-6361:20010192

J. Arons, S.M. Lea, Accretion onto magnetized neutron stars - Structure and interchange instability of a model magnetosphere. ApJ 207, 914-936 (1976). doi:10.1086/154562

M.J. Aschwanden, A Macroscopic Description of Self-Organized Criticality Systems and Astrophysical Applications. ArXiv e-prints (2013)

M. Asplund, N. Grevesse, A.J. Sauval, P. Scott, The chemical composition of the sun. ARA\&A 47, 481-522 (2009)

M.D. Audley, F. Nagase, K. Mitsuda, L. Angelini, R.L. Kelley, ASCA observations of OAO 1657-415 and its dust-scattered X-ray halo. MNRAS 367, 1147-1154 (2006)

J. Babel, Multi-component radiatively driven winds from A and B stars. I. The metallic wind of a main sequence A star. A\&A 301, 823 (1995)

P. Bak, C. Tang, K. Wiesenfeld, Self-organized criticality - An explanation of 1/f noise. Physical Review Letters 59, 381-384 (1987). doi:10.1103/PhysRevLett.59.381

M. Balucinska-Church, D. McCammon, Photoelectric absorption cross sections with variable abundances. ApJ 400, 699 (1992)

R. Barba, R. Gamen, N. Morrell, HD 74194, a new binary supergiant fast X-ray transient?, possible optical counterpart of INTEGRAL hard X-ray source IGR J08408-4503. The Astronomer's Telegram 819, 1 (2006)

M.M. Basko, R.A. Sunyaev, Radiative transfer in a strong magnetic field and accreting Xray pulsars. A\&A 42, 311-321 (1975)

B. Bates, S. Gilheany, IUE observations of mass-loss spectral features in B5-B9 supergiants. MNRAS 243, 320-329 (1990)

P.A. Becker, M.T. Wolff, Thermal and bulk comptonization in accretion-powered x-ray pulsars. ApJ 654, 435-457 (2007)

P.A. Becker, D. Klochkov, G. Schönherr, O. Nishimura, C. Ferrigno, I. Caballero, P. Kretschmar, M.T. Wolff, J. Wilms, R. Staubert, Spectral formation in accreting X-ray pulsars: bimodal variation of the cyclotron energy with luminosity. A\&A 544, 123 (2012)

A.M. Beloborodov, Gravitational bending of light near compact objects. ApJ 566, 85-88 (2002)

I.B. Bernstein, E.A. Frieman, M.D. Kruskal, R.M. Kulsrud, An energy principle for hydromagnetic stability problems. Proceedings of the Royal Society of London. Series A, Mathematical and Physical Sciences 244(1236), 17-40 (1958)

V. Bhalerao, P. Romano, J. Tomsick, L. Natalucci, D.M. Smith, E. Bellm, S.E. Boggs, D. Chakrabarty, F.E. Christensen, W.W. Craig, F. Fuerst, C.J. Hailey, F.A. Harrison, R.A. Krivonos, T.-N. Lu, K. Madsen, D. Stern, G. Younes, W. Zhang, NuSTAR detection of a cyclotron line in the supergiant fast X-ray transient IGR J17544-2619. MNRAS 447, 2274-2281 (2015). doi:10.1093/mnras/stu2495 
A.J. Bird, A. Bazzano, A.B. Hill, V.A. McBride, V. Sguera, S.E. Shaw, H.J. Watkins, Discovery of a 30-d period in the supergiant fast X-ray transient SAX J1818.6-1703. MNRAS 393, 11-15 (2009). doi:10.1111/j.1745-3933.2008.00583.x

J.M. Blondin, J.W. Woo, Wind dynamics in SMC X-1. 1: Hydrodynamic simulation. ApJ 445, 889-895 (1995). doi:10.1086/175748

J.M. Blondin, T.R. Kallman, B.A. Fryxell, R.E. Taam, Hydrodynamic simulations of stellar wind disruption by a compact X-ray source. ApJ 356, 591-608 (1990). doi:10.1086/168865

J.M. Blondin, I.R. Stevens, T.R. Kallman, Enhanced winds and tidal streams in massive X-ray binaries. ApJ 371, 684-695 (1991)

A. Bodaghee, R. Walter, J.A. Zurita Heras, A.J. Bird, T.J.-L. Courvoisier, A. Malizia, R. Terrier, P. Ubertini, IGR J16393-4643: a new heavily-obscured X-ray pulsar. A\&A 447, 1027-1034 (2006). doi:10.1051/0004-6361:20053809

A. Bodaghee, J.A. Tomsick, J. Rodriguez, S. Chaty, K. Pottschmidt, R. Walter, P. Romano, Suzaku Observes Weak Flares from IGR J17391-3021 Representing a Common Low-activity State in this Supergiant Fast X-ray Transient. ApJ 727, 59 (2011). doi:10.1088/0004-637X/727/1/59

A. Bodaghee, F. Rahoui, J.A. Tomsick, J. Rodriguez, Chandra Observations of Five INTEGRAL Sources: New X-Ray Positions for IGR J16393-4643 and IGR J17091-3624. ApJ 751, 113 (2012). doi:10.1088/0004-637X/751/2/113

H. Bondi, F. Hoyle, On the mechanism of accretion by stars. MNRAS 104, 273-282 (1944)

B. Boroson, S.D. Vrtilek, T. Kallman, M. Corcoran, Chandra Grating Spectroscopy of the X-Ray Binary $4 \mathrm{U}$ 1700-37 in a Flaring State. ApJ 592, 516-531 (2003). doi: $10.1086 / 375636$

J.-C. Bouret, T. Lanz, D.J. Hillier, Lower mass loss rates in O-type stars: Spectral signatures of dense clumps in the wind of two Galactic O4 stars. A\&A 438, 301-316 (2005). doi:10.1051/0004-6361:20042531

J.-C. Bouret, D.J. Hillier, T. Lanz, A.W. Fullerton, Properties of Galactic early-type O-supergiants. A combined FUV-UV and optical analysis. A\&A 544, 67 (2012). doi:10.1051/0004-6361/201118594

E. Bozzo, M. Falanga, L. Stella, Are There Magnetars in High-Mass X-Ray Binaries? The Case of Supergiant Fast X-Ray Transients. ApJ 683, 1031-1044 (2008). doi: $10.1086 / 589990$

E. Bozzo, L. Stella, C. Ferrigno, A. Giunta, M. Falanga, S. Campana, G. Israel, J.C. Leyder, The supergiant fast X-ray transients XTE J1739-302 and IGR J08408-4503 in quiescence with XMM-Newton. A\&A 519, 6 (2010). doi:10.1051/0004-6361/201014095

E. Bozzo, A. Giunta, G. Cusumano, C. Ferrigno, R. Walter, S. Campana, M. Falanga, G. Israel, L. Stella, XMM-Newton observations of IGR J18410-0535: the ingestion of a clump by a supergiant fast X-ray transient. A\&A 531, 130 (2011). doi:10.1051/00046361/201116726

E. Bozzo, L. Pavan, C. Ferrigno, M. Falanga, S. Campana, S. Paltani, L. Stella, R. Walter, XMM-Newton observations of four high mass X-ray binaries and IGR J17348-2045. A\&A 544, 118 (2012). doi:10.1051/0004-6361/201218900

E. Bozzo, P. Romano, L. Ducci, F. Bernardini, M. Falanga, Supergiant fast X-ray transients as an under-luminous class of supergiant X-ray binaries. Advances in Space Research 55, 1255-1263 (2015). doi:10.1016/j.asr.2014.11.012

E. Bozzo, V. Bhalerao, P. Pradhan, J. Tomsick, P. Romano, C. Ferrigno, S. Chaty, L. Oskinova, A. Manousakis, R. Walter, M. Falanga, S. Campana, L. Stella, M. Ramolla, R. Chini, Multi-wavelength observations of IGR J17544-2619 from quiescence to outburst. 
ArXiv e-prints (2016)

D.J. Burnard, J. Arons, S.M. Lea, Accretion onto magnetized neutron stars - X-ray pulsars with intermediate rotation rates. ApJ 266, 175-187 (1983). doi:10.1086/160768

K. Butler, J.R. Giddings, notitle. Newsl. Anal. Astron. Spectra 9 (1985)

J.P. Cassinelli, N.A. Miller, W.L. Waldron, J.J. MacFarlane, D.H. Cohen, Chandra Detection of Doppler-shifted X-Ray Line Profiles from the Wind of $\zeta$ Puppis (O4 F). ApJ 554, 5558 (2001). doi:10.1086/320916

J.I. Castor, D.C. Abbott, R.I. Klein, Radiation-driven winds in Of stars. ApJ 195, 157-174 (1975)

S. Chaty, F. Rahoui, C. Foellmi, J.A. Tomsick, J. Rodriguez, R. Walter, Multi-wavelength observations of Galactic hard X-ray sources discovered by INTEGRAL. I. The nature of the companion star. A\&A 484, 783-800 (2008). doi:10.1051/0004-6361:20078768

D.J. Clark, A.B. Hill, A.J. Bird, V.A. McBride, S. Scaringi, A.J. Dean, Discovery of the orbital period in the supergiant fast X-ray transient IGR J17544-2619. MNRAS 399, 113117 (2009). doi:10.1111/j.1745-3933.2009.00737.x

D.J. Clark, V. Sguera, A.J. Bird, V.A. McBride, A.B. Hill, S. Scaringi, S. Drave, A. Bazzano, A.J. Dean, The orbital period in the supergiant fast X-ray transient IGR J16465-4507. MNRAS 406, 75-79 (2010). doi:10.1111/j.1745-3933.2010.00885.x

G.W. Clark, The Orbit of the Binary X-Ray Pulsar 4U 1538-52 from Rossi X-Ray Timing Explorer Observations. ApJ 542, 131-133 (2000). doi:10.1086/312926

G.W. Clark, J.W. Woo, F. Nagase, Properties of a B0 I stellar wind and interstellar grains derived from GINGA observations of the binary X-ray pulsar 4U 1538-52. ApJ 422, 336350 (1994). doi:10.1086/173729

M.J. Coe, J. Fabregat, I. Negueruela, P. Roche, I.A. Steele, Discovery of the optical counterpart to the ASCA transient AX 1845.0-0433. MNRAS 281, 333-338 (1996)

D.H. Cohen, M.A. Leutenegger, E.E. Wollman, J. Zsargó, D.J. Hillier, R.H.D. Townsend, S.P. Owocki, A mass-loss rate determination for $\zeta$ Puppis from the quantitative analysis of X-ray emission-line profiles. MNRAS 405, 2391-2405 (2010a). doi:10.1111/j.13652966.2010.16606.x

D.H. Cohen, M.A. Leutenegger, E.E. Wollman, J. Zsargó, D.J. Hillier, R.H.D. Townsend, S.P. Owocki, A mass-loss rate determination for $\zeta$ Puppis from the quantitative analysis of X-ray emission-line profiles. MNRAS 405, 2391-2405 (2010b). doi:10.1111/j.13652966.2010.16606.x

D.H. Cohen, E.E. Wollman, M.A. Leutenegger, J.O. Sundqvist, A.W. Fullerton, J. Zsargó, S.P. Owocki, Measuring mass-loss rates and constraining shock physics using X-ray line profiles of O stars from the Chandra archive. MNRAS 439, 908-923 (2014). doi:10.1093/mnras/stu008

A. Coleiro, S. Chaty, J.A. Zurita Heras, F. Rahoui, J.A. Tomsick, Infrared identification of high-mass X-ray binaries discovered by INTEGRAL. A\&A 560, 108 (2013). doi:10.1051/0004-6361/201322382

R.H.D. Corbet, H.A. Krimm, Superorbital Periodic Modulation in Wind-accretion Highmass X-Ray Binaries from Swift Burst Alert Telescope Observations. ApJ 778, 45 (2013). doi:10.1088/0004-637X/778/1/45

R.H.D. Corbet, K. Mukai, The Orbit and Position of the X-Ray Pulsar XTE J1855-026: an Eclipsing Supergiant System. ApJ 577, 923-928 (2002). doi:10.1086/342244

R.H.D. Corbet, D.C. Hannikainen, R. Remillard, The Orbital Period of IGR J19140+098. The Astronomer's Telegram 269, 1 (2004)

R.H.D. Corbet, H.A. Krimm, G.K. Skinner, A 44 Day Period in AX J1700.2-4220 from Swift/BAT Observations. The Astronomer's Telegram 2559, 1 (2010) 
R.H.D. Corbet, F.E. Marshall, A.G. Peele, T. Takeshima, Rossi X-Ray Timing Explorer Observations of the X-Ray Pulsar XTE J1855-026: A Possible New Supergiant System. ApJ 517, 956-963 (1999). doi:10.1086/307235

R.H.D. Corbet, S.D. Barthelmy, W.H. Baumgartner, H.A. Krimm, C.B. Markwardt, G.K. Skinner, J. Tueller, A 10 Day Period in IGR J16328-4726 from Swift/BAT Observations. The Astronomer's Telegram 2588, 1 (2010a)

R.H.D. Corbet, S.D. Barthelmy, W.H. Baumgartner, H.A. Krimm, C.B. Markwardt, G.K. Skinner, J. Tueller, A 6.8 Day Period in IGR J16493-4348 from Swift/BAT and RXTE/PCA Observations. The Astronomer's Telegram 2599, 1 (2010b)

R. Corbet, L. Barbier, S. Barthelmy, J. Cummings, E. Fenimore, N. Gehrels, D. Hullinger, H. Krimm, C. Markwardt, D. Palmer, A. Parsons, T. Sakamoto, G. Sato, J. Tueller, SwiftSurvey Team, Swift/BAT Discovery of the Orbital Period of IGR J16320-4751. The Astronomer's Telegram 649, 1 (2005)

R. Corbet, L. Barbier, S. Barthelmy, J. Cummings, E. Fenimore, N. Gehrels, D. Hullinger, H. Krimm, C. Markwardt, D. Palmer, A. Parsons, T. Sakamoto, G. Sato, J. Tueller, R. Remillard, Swift/BAT and RXTE/ASM Discovery of the Orbital Period of IGR J164184532. The Astronomer's Telegram 779, 1 (2006)

N.L.J. Cox, L. Kaper, M.R. Mokiem, VLT/UVES spectroscopy of the O supergiant companion to ¡ASTROBJ $\underset{i}{ } 4 \mathrm{U}$ 1907+09 /ASTROBJ $_{i}$ (7). A\&A 436, 661-669 (2005). doi:10.1051/0004-6361:20040511

D. Crampton, J.B. Hutchings, A.P. Cowley, The supergiant X-ray binary system 2S $0114+$ 650. ApJ 299, 839-844 (1985). doi:10.1086/163750

S.R. Cranmer, S.P. Owocki, The effect of oblateness and gravity darkening on the radiation driving in winds from rapidly rotating B stars. ApJ 440, 308-321 (1995)

S.R. Cranmer, S.P. Owocki, Hydrodynamical Simulations of Corotating Interaction Regions and Discrete Absorption Components in Rotating O-Star Winds. ApJ 462, 469 (1996)

P.A. Crowther, D.J. Lennon, N.R. Walborn, Physical parameters and wind properties of galactic early B supergiants. A\&A 446, 279-293 (2006)

D.W. Datlowe, M.J. Elcan, H.S. Hudson, OSO-7 observations of solar X-rays in the energy range 10-100 keV. Sol. Phys. 39, 155-174 (1974). doi:10.1007/BF00154978

K. Davidson, J.P. Ostriker, Neutron-star accretion in a stellar wind: Model for a pulsed x-ray source. ApJ 179, 585-598 (1973)

R.E. Davies, J.E. Pringle, Spindown of neutron stars in close binary systems. II. MNRAS 196, 209-224 (1981)

R.E. Davies, A.C. Fabian, J.E. Pringle, Spindown of neutron stars in close binary systems. MNRAS 186, 779-782 (1979)

R.H. Densham, P.A. Charles, Optical photometry and spectroscopy of the X-ray pulsar 1E 1145.1-6141. MNRAS 201, 171-178 (1982)

L. Dessart, S.P. Owocki, Two-dimensional simulations of the line-driven instability in hotstar winds. A\&A 406, 1-4 (2003). doi:10.1051/0004-6361:20030810

L. Dessart, S.P. Owocki, 2D simulations of the line-driven instability in hot-star winds. II. Approximations for the 2D radiation force. A\&A 437, 657-666 (2005). doi:10.1051/0004-6361:20052778

J.M. Dickey, F.J. Lockman, H I in the galaxy. ARA\&A 28, 215 (1990)

A. Domiciano de Souza, P. Kervella, S. Jankov, L. Abe, F. Vakili, E. di Folco, F. Paresce, The spinning-top Be star Achernar from VLTI-VINCI. A\&A 407, 47-50 (2003)

V. Doroshenko, A. Santangelo, V. Suleimanov, Witnessing the magnetospheric boundary at work in Vela X-1. A\&A 529, 52 (2011). doi:10.1051/0004-6361/201116482

V. Doroshenko, A. Santangelo, L. Ducci, D. Klochkov, Supergiant, fast, but not so transient 
4U 1907+09. A\&A 548, 19 (2012). doi:10.1051/0004-6361/201220085

S.P. Drave, D.J. Clark, A.J. Bird, V.A. McBride, A.B. Hill, V. Sguera, S. Scaringi, A. Bazzano, Discovery of the 51.47-d orbital period in the supergiant fast X-ray transient XTE J1739-302 with INTEGRAL. MNRAS 409, 1220-1226 (2010). doi:10.1111/j.13652966.2010.17383.x

S.P. Drave, A.J. Bird, L.J. Townsend, A.B. Hill, V.A. McBride, V. Sguera, A. Bazzano, D.J. Clark, X-ray pulsations from the region of the supergiant fast X-ray transient IGR J17544-2619. A\&A 539, 21 (2012). doi:10.1051/0004-6361/201117947

S.P. Drave, A.J. Bird, M.E. Goossens, L. Sidoli, V. Sguera, M. Fiocchi, A. Bazzano, Confirmation of the superorbital modulation of the high mass X-ray binaries 4U 1909+07, IGR J16479-4514 and IGR J16418-4532 with INTEGRAL/IBIS. The Astronomer's Telegram 5131, 1 (2013a)

S.P. Drave, A.J. Bird, L. Sidoli, V. Sguera, V.A. McBride, A.B. Hill, A. Bazzano, M.E. Goossens, INTEGRAL and XMM-Newton observations of IGR J16418-4532: evidence of accretion regime transitions in a supergiant fast X-ray transient. MNRAS 433, 528-542 (2013b). doi:10.1093/mnras/stt754

S.P. Drave, A.J. Bird, L. Sidoli, V. Sguera, A. Bazzano, A.B. Hill, M.E. Goossens, New insights on accretion in supergiant fast X-ray transients from XMM-Newton and INTEGRAL observations of IGR J17544-2619. MNRAS 439, 2175-2185 (2014). doi:10.1093/mnras/stu110

L. Ducci, L. Sidoli, A. Paizis, INTEGRAL results on supergiant fast X-ray transients and accretion mechanism interpretation: ionization effect and formation of transient accretion discs. MNRAS 408, 1540-1550 (2010). doi:10.1111/j.1365-2966.2010.17216.x

L. Ducci, L. Sidoli, S. Mereghetti, A. Paizis, P. Romano, The structure of blue supergiant winds and the accretion in supergiant high-mass X-ray binaries. MNRAS 398, 2152-2165 (2009). doi:10.1111/j.1365-2966.2009.15265.x

L. Ducci, V. Doroshenko, M. Sasaki, A. Santangelo, P. Esposito, P. Romano, S. Vercellone, Spectral and temporal properties of the supergiant fast X-ray transient IGR J18483-0311 observed by INTEGRAL. A\&A 559, 135 (2013). doi:10.1051/0004-6361/201322299

P.L. Dufton, P.R. Dunstall, C.J. Evans, I. Brott, M. Cantiello, A. de Koter, S.E. de Mink, M. Fraser, V. Hénault-Brunet, I.D. Howarth, N. Langer, D.J. Lennon, N. Markova, H. Sana, W.D. Taylor, The VLT-FLAMES Tarantula Survey: The Fastest Rotating O-type Star and Shortest Period LMC Pulsar - Remnants of a Supernova Disrupted Binary? ApJ 743, 22 (2011). doi:10.1088/2041-8205/743/1/L22

R. Edgar, A review of bondi-hoyle-lyttleton accretion. New Astronomy Reviews 48(10), 843-859 (2004). doi:http://dx.doi.org/10.1016/j.newar.2004.06.001

W. Eikmann, J. Wilms, J. Lee, Monte Carlo simulations of X-ray absorption in the interstellar medium, in An INTEGRAL view of the high-energy sky (the first 10 years). PoS, 2012

W. Eikmann, J. Wilms, R.K. Smith, J.C. Lee, X-rray transmission and reflection through a compton-thick medium via monte-carlo simulations. Ac. Polytechnica 54, 177-182 (2014)

R.F. Elsner, F.K. Lamb, Accretion by magnetic neutron stars. I - Magnetospheric structure and stability. ApJ 215, 897-913 (1977). doi:10.1086/155427

T. Endo, M. Ishida, K. Masai, H. Kunieda, H. Inoue, F. Nagase, Broadening of Nearly Neutral Iron Emission Line of GX 301-2 Observed with ASCA. ApJ 574, 879-898 (2002)

T. Eversberg, S. Lépine, A.F.J. Moffat, Outmoving Clumps in the Wind of the Hot O Supergiant $\zeta$ Puppis. ApJ 494, 799-805 (1998). doi:10.1086/305218

M. Falanga, E. Bozzo, A. Lutovinov, J.M. Bonnet-Bidaud, Y. Fetisova, J. Puls, The 
ephemeris, orbital decay, and masses of 10 eclipsing HMXBs. ArXiv e-prints (2015)

R. Farinelli, C. Ceccobello, P. Romano, L. Titarchuk, Numerical solution of the radiative transfer equation: X-ray spectral formation from cylindrical accretion onto a magnetized neutron star. A\&A 538, 67 (2012a). doi:10.1051/0004-6361/201118008

R. Farinelli, P. Romano, V. Mangano, C. Ceccobello, L. Ducci, S. Vercellone, P. Esposito, J.A. Kennea, D.N. Burrows, Swift observations of two supergiant fast X-ray transient prototypes in outburst. MNRAS 424, 2854-2863 (2012b). doi:10.1111/j.13652966.2012.21422.x

S.A. Farrell, R.K. Sood, P.M. O’Neill, Super-orbital period in the high-mass X-ray binary 2S 0114+650. MNRAS 367, 1457-1462 (2006). doi:10.1111/j.1365-2966.2006.10150.x

S.A. Farrell, R.K. Sood, P.M. O'Neill, S. Dieters, A detailed study of 2S 0114+650 with the Rossi X-ray Timing Explorer. MNRAS 389, 608-628 (2008). doi:10.1111/j.13652966.2008.13588.x

U. Feldman, Elemental abundances in the upper solar atmosphere. Physica Scripta 46, 202220 (1992)

A. Feldmeier, Time-dependent structure and energy transfer in hot star winds. A\&A 299, $523(1995)$

A. Feldmeier, I. Shlosman, Runaway of Line-driven Winds toward Critical and Overloaded Solutions. ApJ 532, 125-128 (2000)

A. Feldmeier, I. Shlosman, Abbott Wave-triggered Runaway in Line-driven Winds from Stars and Accretion Disks. ApJ 564, 385-394 (2002)

A. Feldmeier, L. Oskinova, W.-R. Hamann, X-ray line emission from a fragmented stellar wind. A\&A 403, 217-224 (2003). doi:10.1051/0004-6361:20030231

A. Feldmeier, J. Puls, A.W.A. Pauldrach, A possible origin for X-rays from O stars. A\&A 322, 878-895 (1997a)

A. Feldmeier, R. Kudritzki, R. Palsa, A.W.A. Pauldrach, J. Puls, The X-ray emission from shock cooling zones in O star winds. A\&A 320, 899-912 (1997b)

P. Filliatre, S. Chaty, The Optical/Near-Infrared Counterpart of the INTEGRAL Obscured Source IGR J16318-4848: An sgB[e] in a High-Mass X-Ray Binary? ApJ 616, 469-484 (2004). doi:10.1086/424869

M. Fiocchi, V. Sguera, A. Bazzano, L. Bassani, A.J. Bird, L. Natalucci, P. Ubertini, IGR J16328-4726: A New Candidate Supergiant Fast X-ray Transient. ApJ 725, 68-72 (2010). doi:10.1088/2041-8205/725/1/L68

M. Fiocchi, A. Bazzano, A.J. Bird, S.P. Drave, L. Natalucci, P. Persi, L. Piro, P. Ubertini, The INTEGRAL Source IGR J16328-4726: A High-mass X-Ray Binary from the BeppoSAX Era. ApJ 762, 19 (2013). doi:10.1088/0004-637X/762/1/19

J. Frank, A. King, D.J. Raine, Accretion Power in Astrophysics: Third Edition 2002

C. Fransson, A.C. Fabian, X-ray induced shocks in stellar winds. A\&A 87, 102-108 (1980)

D.B. Friend, D.C. Abbott, The theory of radiatively driven stellar winds. III - Wind models with finite disk correction and rotation. ApJ 311, 701-707 (1986)

D.B. Friend, J.I. Castor, Radiation-driven winds in X-ray binaries. ApJ 261, 293-300 (1982). doi: $10.1086 / 160340$

D.B. Friend, J.I. Castor, Stellar winds driven by multiline scattering. ApJ 272, 259-272 (1983)

S. Fritz, I. Kreykenbohm, J. Wilms, R. Staubert, F. Bayazit, K. Pottschmidt, J. Rodriguez, A. Santangelo, A torque reversal of 4U 1907+09. A\&A 458, 885-893 (2006). doi:10.1051/0004-6361:20065557

A.W. Fullerton, S.P. Owocki, Can Nonstationary Velocity Plateaus Account for Slowly Moving Discrete Absorption Components? (Contributed Poster), in Nonisotropic and Variable 
Outflows from Stars, ed. by L. Drissen, C. Leitherer, A. Nota Astronomical Society of the Pacific Conference Series, vol. 22, 1992, p. 177

A.W. Fullerton, D.L. Massa, R.K. Prinja, The Discordance of Mass-Loss Estimates for Galactic O-Type Stars. ApJ 637, 1025-1039 (2006). doi:10.1086/498560

A.W. Fullerton, D.L. Massa, R.K. Prinja, S.P. Owocki, S.R. Cranmer, Wind variability of B supergiants. III. Corotating spiral structures in the stellar wind of HD 64760. A\&A 327, 699-720 (1997)

F. Fürst, I. Kreykenbohm, K. Pottschmidt, J. Wilms, M. Hanke, R.E. Rothschild, P. Kretschmar, N.S. Schulz, D.P. Huenemoerder, D. Klochkov, R. Staubert, X-ray variation statistics and wind clumping in Vela X-1. A\&A 519, 37 (2010). doi:10.1051/00046361/200913981

F. Fürst, S. Suchy, I. Kreykenbohm, L. Barragán, J. Wilms, K. Pottschmidt, I. Caballero, P. Kretschmar, C. Ferrigno, R.E. Rothschild, Study of the many fluorescent lines and the absorption variability in GX 301-2 with XMM-Newton. A\&A 535, 9 (2011). doi:10.1051/0004-6361/201117665

F. Fürst, K. Pottschmidt, J. Wilms, J.A. Tomsick, M. Bachetti, S.E. Boggs, F.E. Christensen, W.W. Craig, B.W. Grefenstette, C.J. Hailey, F. Harrison, K.K. Madsen, J.M. Miller, D. Stern, D.J. Walton, W. Zhang, NuSTAR Discovery of a Luminosity Dependent Cyclotron Line Energy in Vela X-1. ApJ 780, 133 (2014). doi:10.1088/0004-637X/780/2/133

R. Gabler, A. Gabler, R.P. Kudritzki, J. Puls, A. Pauldrach, Unified NLTE model atmospheres including spherical extension and stellar winds - Method and first results. A\&A 226, 162-182 (1989)

R. Gamen, R.H. Barbà, N.R. Walborn, N.I. Morrell, J.I. Arias, J. Maíz Apellániz, A. Sota, E.J. Alfaro, The eccentric short-period orbit of the supergiant fast X-ray transient HD 74194 (=LM Vel). A\&A 583, 4 (2015). doi:10.1051/0004-6361/201527140

K.G. Gayley, S.P. Owocki, S.R. Cranmer, Momentum deposition on Wolf-Rayet winds: Nonisotropic diffusion with effective gray opacity. ApJ 442, 296-310 (1995)

J.R. Giddings, PhD thesis, University of London, 1981

A. Giménez-García, J.M. Torrejón, W. Eikmann, S. Martínez-Núñez, L.M. Oskinova, J.J. Rodes-Roca, G. Bernabéu, An XMM-Newton view of FeK $\alpha$ in high-mass X-ray binaries. A\&A 576, 108 (2015). doi:10.1051/0004-6361/201425004

A. Giménez-García, T. Shenar, J.M. Torrejón, L. Oskinova, S. Martínez-Núñez, W.R. Hamann, J.J. Rodes-Roca, A. González-Galán, J. Alonso-Santiago, C. GonzálezFernández, G. Bernabeu, A. Sander, Measuring the stellar wind parameters in IGR J17544-2619 and Vela X-1 constrains the accretion physics in supergiant fast X-ray transient and classical supergiant X-ray binaries. A\&A 591, 26 (2016). doi:10.1051/0004$6361 / 201527551$

R. González-Riestra, T. Oosterbroek, E. Kuulkers, A. Orr, A.N. Parmar, XMM-Newton observations of the INTEGRAL X-ray transient IGR J17544-2619. A\&A 420, 589-594 (2004). doi:10.1051/0004-6361:20035940

M.E. Goossens, A.J. Bird, S.P. Drave, A. Bazzano, A.B. Hill, V.A. McBride, V. Sguera, L. Sidoli, Discovering a 5.72-d period in the supergiant fast X-ray transient AX J1845.00433. MNRAS 434, 2182-2187 (2013). doi:10.1093/mnras/stt1166

E. Göğüş, I. Kreykenbohm, T.M. Belloni, Discovery of a peculiar dip from GX 301-2. A\&A 525, 6 (2011). doi:10.1051/0004-6361/201015905

G. Gräfener, W.-R. Hamann, Hydrodynamic model atmospheres for WR stars. Selfconsistent modeling of a WC star wind. A\&A 432, 633-645 (2005). doi:10.1051/00046361:20041732

G. Gräfener, W.-R. Hamann, The metallicity dependence of WR wind models, in Stellar 
Evolution at Low Metallicity: Mass Loss, Explosions, Cosmology, ed. by H.J.G.L.M. Lamers, N. Langer, T. Nugis, K. Annuk Astronomical Society of the Pacific Conference Series, vol. 353, 2006, p. 171

G. Gräfener, W.-R. Hamann, Hydrodynamic Model Atmospheres for WR Stars: First Results and Their Consequences for Interacting Winds in Massive Binary Systems, in Massive Stars in Interactive Binaries, ed. by N. St.-Louis, A.F.J. Moffat Astronomical Society of the Pacific Conference Series, vol. 367, 2007, p. 131

G. Gräfener, L. Koesterke, W.-R. Hamann, Line-blanketed model atmospheres for WR stars. A\&A 387, 244-257 (2002). doi:10.1051/0004-6361:20020269

S.A. Grebenev, R.A. Sunyaev, The first observation of AX J1749.1-2733 in a bright X-ray state. Another fast transient revealed by INTEGRAL. Astronomy Letters 33, 149-158 (2007). doi:10.1134/S1063773707030024

N. Grevesse, A.J. Sauval, Standard solar composition. Space Sci. Rev. 85, 161-174 (1998)

V. Grinberg, M.A. Leutenegger, N. Hell, K. Pottschmidt, M. Böck, J.A. García, M. Hanke, M.A. Nowak, J.O. Sundqvist, R.H.D. Townsend, J. Wilms, Long term variability of Cygnus X-1. VII. Orbital variability of the focussed wind in Cyg X-1/HDE 226868 system. A\&A 576, 117 (2015). doi:10.1051/0004-6361/201425418

M.A.T. Groenewegen, H.J.G.L.M. Lamers, A.W.A. Pauldrach, The winds of O-stars. II The terminal velocities of stellar winds of O-type stars. A\&A 221, 78-88 (1989)

J.H. Groh, G. Meynet, S. Ekström, C. Georgy, The evolution of massive stars and their spectra. I. A non-rotating $60 \mathrm{M}_{\odot}$ star from the zero-age main sequence to the pre-supernova stage. A\&A 564, 30 (2014). doi:10.1051/0004-6361/201322573

T. Guver, F. Ozel, H. Marshall, D. Psaltis, M. Guainazzi, M. Diaz-Trigo, Systematic Uncertainties in the Spectroscopic Measurements of Neutron-Star Masses and Radii from Thermonuclear X-ray Bursts. III. Absolute Flux Calibration. ArXiv e-prints (2015)

V.V. Gvaramadze, N. Langer, J. Mackey, $\zeta$ Oph and the weak-wind problem. MNRAS 427, 50-54 (2012). doi:10.1111/j.1745-3933.2012.01343.x

R. Hainich, U. Rühling, H. Todt, L.M. Oskinova, A. Liermann, G. Gräfener, C. Foellmi, O. Schnurr, W.-R. Hamann, The Wolf-Rayet stars in the Large Magellanic Cloud. A comprehensive analysis of the WN class. A\&A 565, 27 (2014). doi:10.1051/0004$6361 / 201322696$

W.-R. Hamann, Line formation in expanding atmospheres - On the validity of the Sobolev approximation. A\&A 93, 353-361 (1981)

W.-R. Hamann, G. Gräfener, Grids of model spectra for WN stars, ready for use. A\&A 427, 697-704 (2004). doi:10.1051/0004-6361:20040506

W.-R. Hamann, L. Koesterke, Spectrum formation in clumped stellar winds: consequences for the analyses of Wolf-Rayet spectra. A\&A 335, 1003-1008 (1998)

W.-R. Hamann, A. Feldmeier, L.M. Oskinova (eds.), Clumping in hot-star winds, in Clumping in Hot-Star Winds 2008

W.-R. Hamann, J.C. Brown, A. Feldmeier, L.M. Oskinova, On the wavelength drift of spectral features from structured hot star winds. A\&A 378, 946-953 (2001)

M. Hanke, Probing the Environment of Accreting Compact Objects, PhD thesis, Dr. Karl Remeis-Sternwarte, Astronomisches Institut der Universität Erlangen-Nürnberg, Sternwartstr. 7, 96049 Bamberg, Germany, 2011

M. Hanke, J. Wilms, M.A. Nowak, K. Pottschmidt, N.S. Schulz, J.C. Lee, Chandra X-Ray Spectroscopy of the Focused Wind in the Cygnus X-1 System. I. The Nondip Spectrum in the Low/Hard State. ApJ 690, 330-346 (2009)

D.C. Hannikainen, M.G. Rawlings, P. Muhli, O. Vilhu, J. Schultz, J. Rodriguez, The nature of the infrared counterpart of IGR J19140+0951. MNRAS 380, 665-668 (2007). 
doi:10.1111/j.1365-2966.2007.12092.X

M.M. Hanson, P.S. Conti, M.J. Rieke, A Spectral Atlas of Hot, Luminous Stars at 2 Microns. ApJS 107, 281 (1996). doi:10.1086/192366

A.K. Harding, Emission Processes in X-ray Pulsars, in The Evolution of X-ray Binariese, ed. by S. Holt, C.S. Day American Institute of Physics Conference Series, vol. 308, 1994, p. 429

A.K. Harding, M. Leventhal, Can accretion onto isolated neutron stars produce gamma-ray bursts? Nature 357, 388 (1992). doi:10.1038/357388a0

S. Hatchett, R. McCray, X-ray sources in stellar winds. ApJ 211, 552-561 (1977). doi:10.1086/154962

P.H. Hauschildt, A fast operator perturbation method for the solution of the special relativistic equation of radiative transfer in spherical symmetry. Journal of Quantitative Spectroscopy and Radiative Transfer 47, 433-453 (1992)

S. Hayakawa, Sudden disappearance of X-ray emission from Vela X-1 - Can this be due to eclipse by a planet of HD77581?, in Proceedings of the Symposium on High-Energy Astrophysics and Cosmology, Pamporovo, Bulgaria, July 18-23, 1983. Adv. Space Res., vol. 3, 1984, pp. 35-38

H.F. Henrichs, L. Kaper, G.A.A. Zwarthoed, Rapid variability in O star winds, in A Decade of UV Astronomy with the IUE Satellite, Volume 2, vol. 2, ed. by E.J. Rolfe, 1988, pp. 145-149

A. Hervé, G. Rauw, Y. Nazé, A detailed X-ray investigation of $\zeta$ Puppis. III. Spectral analysis of the whole RGS spectrum. A\&A 551, 83 (2013). doi:10.1051/0004$6361 / 201219734$

D.J. Hillier, The effects of electron scattering and wind clumping for early emission line stars. A\&A 247, 455-468 (1991)

D.J. Hillier, D.L. Miller, The Treatment of Non-LTE Line Blanketing in Spherically Expanding Outflows. ApJ 496, 407 (1998)

D.J. Hillier, R.P. Kudritzki, A.W. Pauldrach, D. Baade, J.P. Cassinelli, J. Puls, J.H.M.M. Schmitt, The 0.1-2.5-KEV X-Ray Spectrum of the O4F-STAR Zeta-Puppis. A\&A 276, 117 (1993)

C. Ho, J. Arons, High-luminosity accretion in wind-driven binary X-ray sources. ApJ 316, 283-293 (1987). doi:10.1086/165200

I.D. Howarth, R.K. Prinja, The stellar winds of 203 Galactic O stars - A quantitative ultraviolet survey. ApJS 69, 527-592 (1989)

I.D. Howarth, Intrinsic Stellar-Wind Variability (Invited Paper), in Nonisotropic and Variable Outflows from Stars, ed. by L. Drissen, C. Leitherer, A. Nota Astronomical Society of the Pacific Conference Series, vol. 22, 1992, p. 155

I.D. Howarth, R.K. Prinja, D. Massa, The IUE MEGA Campaign: The Rotationally Modulated Wind of zeta Puppis. ApJ 452, 65 (1995)

F. Hoyle, R.A. Lyttleton, The effect of interstellar matter on climatic variation. Mathematical Proceedings of the Cambridge Philosophical Society 35, 405-415 (1939). doi:10.1017/S0305004100021150

I. Hubeny, Non-LTE line-blanketed model atmospheres of hot stars, in 1997 Pacific Rim Conference on Stellar Astrophysics, ed. by K.L. Chan, K.S. Cheng, H.P. Singh Astronomical Society of the Pacific Conference Series, vol. 138, 1998, p. 139

S. Hubrig, L.M. Oskinova, M. Schöller, First detection of a magnetic field in the fast rotating runaway Oe star $\zeta$ Ophiuchi. Astronomische Nachrichten 332, 147 (2011). doi:10.1002/asna.201111516

S. Hubrig, M. Schöller, I. Ilyin, N.V. Kharchenko, L.M. Oskinova, N. Langer, J.F. González, 
A.F. Kholtygin, M. Briquet, Magori Collaboration, Exploring the origin of magnetic fields in massive stars. II. New magnetic field measurements in cluster and field stars. A\&A 551, 33 (2013). doi:10.1051/0004-6361/201220721

D.P. Huenemoerder, L.M. Oskinova, R. Ignace, W.L. Waldron, H. Todt, K. Hamaguchi, S. Kitamoto, On the Weak-wind Problem in Massive Stars: X-Ray Spectra Reveal a Massive Hot Wind in $\mu$ Columbae. ApJ 756, 34 (2012). doi:10.1088/2041-8205/756/2/L34

A. Ibarra, G. Matt, M. Guainazzi, E. Kuulkers, E. Jiménez-Bailón, J. Rodriguez, F. Nicastro, R. Walter, The XMM-Newton/INTEGRAL monitoring campaign of IGR J16318-4848. A\&A 465, 501-507 (2007). doi:10.1051/0004-6361:20066225

R. Ignace, Theoretical Profile Shapes for Optically Thin X-Ray Emission Lines from Spherical Stellar Winds. ApJ 549, 119-123 (2001). doi:10.1086/319141

N.R. Ikhsanov, On the duration of the subsonic propeller state of neutron stars in windfed mass-exchange close binary systems. A\&A 368, 5-7 (2001a). doi:10.1051/00046361:20010140

N.R. Ikhsanov, On the origin of quiescent X-ray emission from A0535+26. A\&A 367, 549556 (2001b). doi:10.1051/0004-6361:20000464

N.R. Ikhsanov, On the state of low luminous accreting neutron stars. A\&A 375, 944-949 (2001c). doi:10.1051/0004-6361:20010909

N.R. Ikhsanov, Neutron Stars in the Subsonic Propeller Stage. Astrophysics 48, 400-410 (2005). doi:10.1007/s10511-005-0039-y

N.R. Ikhsanov, The origin of long-period X-ray pulsars. MNRAS 375, 698-704 (2007). doi:10.1111/j.1365-2966.2006.11331.x

N.R. Ikhsanov, L.A. Pustil'nik, Stability of the magnetospheric boundary of a neutron star undergoing spherical accretion. A\&A 312, 338-344 (1996)

A.F. Illarionov, R.A. Sunyaev, Why the number of galactic x-ray stars is so small? A\&A 39, 185-195 (1975)

H. Inoue, Y. Ogawara, T. Ohashi, I. Waki, S. Hayakawa, H. Kunieda, F. Nagase, H. Tsunemi, Sudden disappearance of Vela X-1 pulses. PASJ 36, 709-713 (1984)

J.J.M. in't Zand, Chandra observation of the fast X-ray transient IGR J17544-2619: evidence for a neutron star? A\&A 441, 1-4 (2005). doi:10.1051/0004-6361:200500162

J.J.M. in't Zand, A. Baykal, T.E. Strohmayer, Recent X-Ray Measurements of the Accretion-powered Pulsar 4U 1907+09. ApJ 496, 386-394 (1998). doi:10.1086/305362

J.J.M. in't Zand, T.E. Strohmayer, A. Baykal, Dipping Activity in the X-Ray Pulsar 4U 1907+09. ApJ 479, 47-50 (1997). doi:10.1086/310570

C. Jain, B. Paul, A. Dutta, Discovery of a short orbital period in the Supergiant Fast X-ray Transient IGR J16479-4514. MNRAS 397, 11-15 (2009). doi:10.1111/j.17453933.2009.00668.x

C. Jain, B. Paul, C. Maitra, Detection of periodic X-ray modulation of SFXT IGR J162075129 in the Swift-BAT light curve. The Astronomer's Telegram 3785, 1 (2011)

C. Jones, W. Forman, H. Tananbaum, E. Schreier, H. Gursky, E. Kellogg, R. Giacconi, Evidence for the Binary Nature of 2u 1700-37. ApJ 181, 43 (1973). doi:10.1086/181181

J.S. Kaastra, R. Mewe, X-ray emission from thin plasmas. I - Multiple Auger ionisation and fluorescence processes for Be to Zn. A\&AS 97, 443-482 (1993)

S.M. Kahn, M.A. Leutenegger, J. Cottam, G. Rauw, J.-M. Vreux, A.J.F. den Boggende, R. Mewe, M. Güdel, High resolution X-ray spectroscopy of zeta Puppis with the XMMNewton reflection grating spectrometer. A\&A 365, 312-317 (2001). doi:10.1051/00046361:20000093

P.M.W. Kalberla, W.B. Burton, D. Hartmann, E.M. Arnal, E. Bajaja, R. Morras, W.G.L. Pöppel, The leiden/argentine/bonn (lab) survey of galactic hi. final data release of the 
combined lds and iar surveys with improved stray-radiation corrections. A\&A 440, 775$782(2005)$

T.R. Kallman, N.E. White, The anomalous X-ray absorption spectrum of Vela X-1. ApJ 261, 35-39 (1982)

T.R. Kallman, P. Palmeri, M.A. Bautista, C. Mendoza, J.H. Krolik, Photoionization Modeling and the K Lines of Iron. ApJS 155, 675-701 (2004)

L. Kaper, G. Hammerschlag-Hensberge, E.J. Zuiderwijk, Spectroscopic evidence for photoionization wakes in VELA X-1 and 4U 1700-37. A\&A 289, 846-854 (1994)

L. Kaper, A. van der Meer, F. Najarro, VLT/UVES spectroscopy of Wray 977, the hypergiant companion to the X-ray pulsar ¡ASTROBJ $i$ GX301-2¡/ASTROBJ ${ }_{i}$. A\&A 457, 595-610 (2006). doi:10.1051/0004-6361:20065393

L. Kaper, H.F. Henrichs, J.S. Nichols, J.H. Telting, Long- and short-term variability in Ostar winds. II. Quantitative analysis of DAC behaviour. A\&A 344, 231-262 (1999)

S. Karino, Bimodality of wind-fed accretion in high-mass X-ray binaries. PASJ 66, 34 (2014). doi:10.1093/pasj/psu018

A. Kaufer, O. Stahl, R.K. Prinja, D. Witherick, Multi-periodic photospheric pulsations and connected wind structures in ASTROBJ $_{i}$ HD 64760;/ASTROBJ $\underset{i}{ }$. A\&A 447, 325-341 (2006)

Z. Keszthelyi, Master Thesis. The Impact of Mass Loss on the Early Evolution of Massive Stars (Ludwig-Maximilians-Universität München, ???, 2015)

H.A. Kobulnicky, I.J. Gilbert, D.C. Kiminki, OB Stars and Stellar Bow shocks in CygnusX: A Novel Laboratory Estimating Stellar Mass Loss Rates. ApJ 710, 549-566 (2010). doi:10.1088/0004-637X/710/1/549

D.T. Koh, L. Bildsten, D. Chakrabarty, R.W. Nelson, T.A. Prince, B.A. Vaughan, M.H. Finger, R.B. Wilson, B.C. Rubin, Rapid Spin-Up Episodes in the Wind-fed Accreting Pulsar GX 301-2. ApJ 479, 933-947 (1997)

R.H. Kramer, D.H. Cohen, S.P. Owocki, X-Ray Emission-Line Profile Modeling of O Stars: Fitting a Spherically Symmetric Analytic Wind-Shock Model to the Chandra Spectrum of $\zeta$ Puppis. ApJ 592, 532-538 (2003). doi:10.1086/375390

U. Kraus, Hollow Accretion Columns on Neutron Stars and the Effects of Gravitational Light Bending. ApJ 563, 289-300 (2001). doi:10.1086/323791

P. Kretschmar, I. Kreykenbohm, J. Wilms, R. Staubert, W. Heindl, D. Gruber, R. Rothschild, Disappearing Pulses in Vela X-1, in Proceedings of the Fifth Compton Symposium, ed. by M.L. McConnell, J.M. Ryan American Institute of Physics Conference Series, vol. 510, 2000, pp. 163-167

I. Kreykenbohm, J. Wilms, P. Kretschmar, J.M. Torrejón, K. Pottschmidt, M. Hanke, A. Santangelo, C. Ferrigno, R. Staubert, High variability in Vela X-1: giant flares and off states. A\&A 492, 511-525 (2008). doi:10.1051/0004-6361:200809956

I. Kreykenbohm, P. Kretschmar, J. Wilms, R. Staubert, E. Kendziorra, D.E. Gruber, W.A. Heindl, R.R. Rothschild, Vela X-1 as seen by rxte. A\&A 341, 141-150 (1999)

J.H. Krolik, T.R. Kallman, Soft X-ray opacity in hot and photoionized gases. ApJ 286, 366370 (1984). doi:10.1086/162608

J. Krticka, J. Kubat, I. Krtickova, X-ray irradiation of the winds in binaries with massive components. ArXiv e-prints (2015)

J. Krtička, NLTE models of line-driven stellar winds - II. O stars in the Small Magellanic Cloud. MNRAS, 266 (2006)

J. Krtička, J. Kubát, Isothermal two-component stellar wind of hot stars. A\&A 359, 983-990 (2000)

J. Krtička, J. Kubát, Multicomponent radiatively driven stellar winds. I. Nonisothermal 
three-component wind of hot B stars. A\&A 369, 222-238 (2001). doi:10.1051/00046361:20010121

J. Krtička, J. Kubát, NLTE models of line-driven stellar winds. I. Method of calculation and first results for O stars. A\&A 417, 1003-1016 (2004). doi:10.1051/0004-6361:20034030

J. Krtička, J. Kubát, J. Skalický, X-Ray Photoionized Bubble in the Wind of Vela X-1 Pulsar Supergiant Companion. ApJ 757, 162 (2012). doi:10.1088/0004-637X/757/2/162

J. Krtička, S.P. Owocki, J. Kubát, R.K. Galloway, J.C. Brown, On multicomponent effects in stellar winds of stars at extremely low metallicity. A\&A 402, 713-718 (2003)

J. Krtička, A. Feldmeier, L.M. Oskinova, J. Kubát, W.-R. Hamann, X-ray emission from hydrodynamical simulations in non-LTE wind models. A\&A 508, 841-848 (2009). doi:10.1051/0004-6361/200912642

R.P. Kudritzki, Line-driven Winds, Ionizing Fluxes, and Ultraviolet Spectra of Hot Stars at Extremely Low Metallicity. I. Very Massive O Stars. ApJ 577, 389-408 (2002). doi:10.1086/342178

R.-P. Kudritzki, J. Puls, Winds from Hot Stars. ARA\&A 38, 613-666 (2000)

R.-P. Kudritzki, D.J. Lennon, J. Puls, Quantitative Spectroscopy of Luminous Blue Stars in Distant Galaxies, in Science with the VLT, ed. by J.R. Walsh, I.J. Danziger, 1995, p. 246

R.-P. Kudritzki, A. Pauldrach, J. Puls, D.C. Abbott, Radiation-driven winds of hot stars. VI - Analytical solutions for wind models including the finite cone angle effect. A\&A 219, 205-218 (1989)

R.-P. Kudritzki, J. Puls, D.J. Lennon, K.A. Venn, J. Reetz, F. Najarro, J.K. McCarthy, A. Herrero, The wind momentum-luminosity relationship of galactic A- and B-supergiants. A\&A 350, 970-984 (1999)

A.K. Kulkarni, M.M. Romanova, Accretion to magnetized stars through the Rayleigh-Taylor instability: global 3D simulations. MNRAS 386, 673-687 (2008). doi:10.1111/j.13652966.2008.13094.x

V. La Parola, G. Cusumano, P. Romano, A. Segreto, S. Vercellone, G. Chincarini, Detection of an orbital period in the supergiant high-mass X-ray binary IGR J16465-4507 with Swift-BAT. MNRAS 405, 66-70 (2010). doi:10.1111/j.1745-3933.2010.00860.x

H.J.G.L.M. Lamers, J.P. Cassinelli, Introduction to Stellar Winds 1999

H.J.G.L.M. Lamers, D.C. Morton, Mass ejection from the O4f star Zeta Puppis. ApJS 32, 715-736 (1976). doi:10.1086/190413

H.J.G.L.M. Lamers, M. Cerruti-Sola, M. Perinotto, The 'SEI' method for accurate and efficient calculations of line profiles in spherically symmetric stellar winds. ApJ 314, 726738 (1987). doi:10.1086/165100

H.J.G.L.M. Lamers, R. Gathier, T.P. Snow Jr., Narrow components in the profiles of ultraviolet resonance lines - Evidence for a two-component stellar wind for O and B stars. ApJ 258, 186-200 (1982)

T. Lanz, I. Hubeny, A Grid of Non-LTE Line-blanketed Model Atmospheres of O-Type Stars. ApJS 146, 417-441 (2003). doi:10.1086/374373

I.Y. Lapshov, R.A. Sunyaev, M.A. Chichkov, V.V. Dremin, S. Brandt, N. Lund, Two years of observation of the X-ray pulsar Vela X-1 with the watch instrument on the granat observatory. Sov. Astron. Lett. 18, 16-19 (1992)

K. Lefever, J. Puls, T. Morel, C. Aerts, L. Decin, M. Briquet, Spectroscopic determination of the fundamental parameters of 66 B-type stars in the field-of-view of the CoRoT satellite. A\&A 515, 74 (2010). doi:10.1051/0004-6361/200911956

C. Leitherer, C. Robert, L. Drissen, Deposition of mass, momentum, and energy by massive stars into the interstellar medium. ApJ 401, 596-617 (1992). doi:10.1086/172089

M.A. Leutenegger, S.P. Owocki, S.M. Kahn, F.B.S. Paerels, Evidence for the Importance of 
Resonance Scattering in X-Ray Emission Line Profiles of the O Star $\zeta$ Puppis. ApJ 659, 642-649 (2007). doi:10.1086/512031

M.A. Leutenegger, D.H. Cohen, J.O. Sundqvist, S.P. Owocki, Constraints on Porosity and Mass Loss in O-star Winds from the Modeling of X-Ray Emission Line Profile Shapes. ApJ 770, 80 (2013). doi:10.1088/0004-637X/770/1/80

A.M. Levine, R. Corbet, Detection of Additional Periodicities in RXTE ASM Light Curves. The Astronomer's Telegram 940, 1 (2006)

J.-C. Leyder, R. Walter, M. Lazos, N. Masetti, N. Produit, Hard X-ray flares in ¡ASTROBJ $_{i}$ IGR J08408-4503i/ASTROBJ $i$ unveil clumpy stellar winds. A\&A 465, 35-38 (2007). doi:10.1051/0004-6361:20066317

A. Lobel, 3-D radiative transfer modeling of rotational modulations in the blue supergiant $\mathrm{J}$ Puppis, in Massive Stars: From alpha to Omega, 2013

A. Lobel, R. Blomme, Modeling Ultraviolet Wind Line Variability in Massive Hot Stars. ApJ 678, 408-430 (2008)

K. Lodders, Solar system abundances and condensation temperatures of the elements. ApJ 591, 1220-1247 (2003)

J. Lorenzo, I. Negueruela, A.J. Norton, HD 306414, the Optical Counterpart to the Peculiar X-Ray Transient IGR J11215-5952, in High Energy Phenomena in Massive Stars, ed. by J. Martí, P.L. Luque-Escamilla, J.A. Combi Astronomical Society of the Pacific Conference Series, vol. 422, 2010, p. 259

J. Lorenzo, I. Negueruela, N. Castro, A.J. Norton, F. Vilardell, A. Herrero, Astrophysical parameters of the peculiar X-ray transient IGR J11215-5952. A\&A 562, 18 (2014). doi:10.1051/0004-6361/201321913

L.B. Lucy, Wave amplification in line-driven winds. ApJ 284, 351-356 (1984). doi:10.1086/162413

L.B. Lucy, D.C. Abbott, Multiline transfer and the dynamics of Wolf-Rayet winds. ApJ 405, 738-746 (1993)

L.B. Lucy, P.M. Solomon, Mass Loss by Hot Stars. ApJ 159, 879-893 (1970)

A. Lutovinov, J. Rodriguez, M. Revnivtsev, P. Shtykovskiy, Discovery of X-ray pulsations from IGR J16320-4751 = AX J1631.9-4752. A\&A 433, 41-44 (2005). doi:10.1051/00046361:200500092

T.J. Maccarone, T.M. Girard, D.I. Casetti-Dinescu, High proper motion X-ray binaries from the Yale Southern Proper Motion Survey. MNRAS 440, 1626-1633 (2014). doi:10.1093/mnras/stu320

J.J. Macfarlane, J.P. Cassinelli, B.Y. Welsh, P.W. Vedder, J.V. Vallerga, W.L. Waldron, Predicted extreme-ultraviolet line emission for nearby main-sequence B stars. ApJ 380, 564574 (1991). doi:10.1086/170614

A. Manousakis, R. Walter, X-ray wind tomography of the highly absorbed HMXB IGR J17252-3616. A\&A 526, 62 (2011)

A. Manousakis, R. Walter, Origin of the X-ray off-states in Vela X-1. A\&A 575, 58 (2015). doi:10.1051/0004-6361/201321414

W.L.F. Marcolino, J. Bouret, F. Martins, D.J. Hillier, T. Lanz, C. Escolano, Analysis of Galactic late-type O dwarfs: more constraints on the weak wind problem. A\&A 498, 837-852 (2009). doi:10.1051/0004-6361/200811289

N. Markova, The ejection of shells in the stellar wind of P CYG - The most plausible explanation of the Balmer-line radial velocity variations. A\&A 162, 3-5 (1986)

N. Markova, J. Puls, Bright OB stars in the Galaxy. IV. Stellar and wind parameters of early to late B supergiants. A\&A 478, 823-842 (2008)

N. Markova, J. Puls, S. Simón-Díaz, A. Herrero, H. Markov, N. Langer, Spectroscopic and 
physical parameters of Galactic O-type stars. II. Observational constraints on projected rotational and extra broadening velocities as a function of fundamental parameters and stellar evolution. A\&A 562, 37 (2014). doi:10.1051/0004-6361/201322661

S. Martínez-Núñez, J.M. Torrejón, M. Kühnel, P. Kretschmar, M. Stuhlinger, J.J. RodesRoca, F. Fürst, I. Kreykenbohm, A. Martin-Carrillo, A.M.T. Pollock, J. Wilms, The accretion environment in vela $\mathrm{x}-1$ during a flaring period using xmm-newton. A\&A 563, 70 (2014)

S. Martínez-Núñez, A. Sander, A. Gímenez-García, A. Gónzalez-Galán, J.M. Torrejón, C. Gónzalez-Fernández, W.-R. Hamann, The donor star of the X-ray pulsar X1908+075. A\&A 578, 107 (2015). doi:10.1051/0004-6361/201424823

F. Martins, D.J. Hillier, On the formation of C iii 4647-50-51 and C iii 5696 in O star atmospheres. A\&A 545, 95 (2012). doi:10.1051/0004-6361/201219788

N. Masetti, M.L. Pretorius, E. Palazzi, L. Bassani, A. Bazzano, A.J. Bird, P.A. Charles, A.J. Dean, A. Malizia, P. Nkundabakura, J.B. Stephen, P. Ubertini, Unveiling the nature of INTEGRAL objects through optical spectroscopy. III. Observations of seven southern sources. A\&A 449, 1139-1149 (2006). doi:10.1051/0004-6361:20054332

N. Masetti, E. Mason, L. Morelli, S.A. Cellone, V.A. McBride, E. Palazzi, L. Bassani, A. Bazzano, A.J. Bird, P.A. Charles, A.J. Dean, G. Galaz, N. Gehrels, R. Landi, A. Malizia, D. Minniti, F. Panessa, G.E. Romero, J.B. Stephen, P. Ubertini, R. Walter, Unveiling the nature of INTEGRAL objects through optical spectroscopy. VI. A multi-observatory identification campaign. A\&A 482, 113-132 (2008). doi:10.1051/0004-6361:20079332

A.B. Mason, J.S. Clark, A.J. Norton, I. Negueruela, P. Roche, Spectral classification of the mass donors in the high-mass X-ray binaries EXO 1722-363 and OAO 1657-415. A\&A 505, 281-286 (2009)

A.B. Mason, A.J. Norton, J.S. Clark, I. Negueruela, P. Roche, The masses of the neutron and donor star in the high-mass X-ray binary IGR J18027-2016. A\&A 532, 124 (2011). doi:10.1051/0004-6361/201117392

A.B. Mason, J.S. Clark, A.J. Norton, P.A. Crowther, T.M. Tauris, N. Langer, I. Negueruela, P. Roche, The evolution and masses of the neutron star and donor star in the high mass X-ray binary OAO 1657-415. MNRAS 422, 199-206 (2012). doi:10.1111/j.13652966.2012.20596.x

D. Massa, A.W. Fullerton, J.S. Nichols, S.P. Owocki, R.K. Prinja, and 28 co-authors, The IUE MEGA Campaign: Wind Variability and Rotation in Early-Type Stars. ApJ 452, 53 (1995)

D. Massa, A.W. Fullerton, G. Sonneborn, J.B. Hutchings, Constraints on the Ionization Balance of Hot-Star Winds from FUSE Observations of O Stars in the Large Magellanic Cloud. ApJ 586, 996-1018 (2003). doi:10.1086/367786

P. Massey, K.F. Neugent, D.J. Hillier, J. Puls, A Bake-off between CMFGEN and FASTWIND: Modeling the Physical Properties of SMC and LMC O-type Stars. ApJ 768, 6 (2013). doi:10.1088/0004-637X/768/1/6

J.S. Mathis, Interstellar dust and extinction. ARA\&A 28, 37-70 (1990)

E.A. Milne, On the possibility of the emission of high-speed atoms from the sun and stars. MNRAS 86, 459-473 (1926)

I. Miškovičová, M. Hanke, J. Wilms, M.A. Nowak, K. Pottschmidt, N.S. Schulz, Spectroscopy of the Stellar Wind in the Cygnus X-1 System. Acta Polytechnica 51(2), 020000 (2011). doi:eprintid: arXiv:1103.2711

M.R. Mokiem, A. de Koter, J. Puls, A. Herrero, F. Najarro, M.R. Villamariz, Spectral analysis of early-type stars using a genetic algorithm based fitting method. A\&A 441, 711-733 (2005) 
M.R. Mokiem, A. de Koter, J.S. Vink, J. Puls, C.J. Evans, S.J. Smartt, P.A. Crowther, A. Herrero, N. Langer, D.J. Lennon, F. Najarro, M.R. Villamariz, The empirical metallicity dependence of the mass-loss rate of O- and early B-type stars. A\&A 473, 603-614 (2007)

J.D. Monnier, M. Zhao, E. Pedretti, N. Thureau, M. Ireland, P. Muirhead, J.-P. Berger, R. Millan-Gabet, G. Van Belle, T. ten Brummelaar, H. McAlister, S. Ridgway, N. Turner, L. Sturmann, J. Sturmann, D. Berger, Imaging the Surface of Altair. Science 317, 342-345 (2007)

K. Mori, M.A. Ruderman, Isolated Magnetar Spin-Down, Soft X-Ray Emission, and RX J1856.5-3754. ApJ 592, 75-78 (2003). doi:10.1086/377705

L.E. Muijres, A. de Koter, J.S. Vink, J. Krtička, J. Kubát, N. Langer, Predictions of the effect of clumping on the wind properties of O-type stars. A\&A 526, 32 (2011). doi:10.1051/0004-6361/201014290

U. Mukherjee, H. Raichur, B. Paul, S. Naik, N. Bhatt, Orbital Evolution and Orbital Phase Resolved Spectroscopy of the HMXB Pulsar 4U 1538-52 with RXTE-PCA and BeppoSAX. Journal of Astrophysics and Astronomy 27, 411-423 (2006)

D.J. Mullan, Corotating interaction regions in stellar winds. ApJ 283, 303-312 (1984)

D.J. Mullan, Displaced narrow absorption components in the spectra of mass-losing OB stars - Indications of corotating interaction regions? A\&A 165, 157-162 (1986)

F. Nagase, S. Hayakawa, N. Sato, K. Masai, H. Inoue, Circumstellar matter in the VELA X-1/HD 77581 system. PASJ 38, 547-569 (1986)

Y. Nazé, L.M. Oskinova, E. Gosset, A Detailed X-Ray Investigation of $\zeta$ Puppis. II. The Variability on Short and Long Timescales. ApJ 763, 143 (2013). doi:10.1088/0004637X/763/2/143

I. Negueruela, D.M. Smith, Optical counterpart to SAX J1818.6-1703. ATel 831, 1 (2006)

I. Negueruela, D.M. Smith, S. Chaty, HD 306414 and IGR J11215-5952. ATel 470, 1 (2005)

I. Negueruela, J.M. Torrejón, P. Reig, Optical and Infrared characterisation of High Mass Xray Binaries discovered by INTEGRAL, in Proceedings of the 7th INTEGRAL Workshop, 2008

I. Negueruela, D.M. Smith, P. Reig, S. Chaty, J.M. Torrejón, Supergiant Fast X-ray Transients: a new class of high mass X-ray binaries unveiled by INTEGRAL, in Proc. of the "The X-ray Universe 2005”, 26-30 September 2005, El Escorial, Madrid, Spain. Ed. by A. Wilson. ESA SP-604, Volume 1, Noordwijk: ESA Pub. Division, ISBN 92-9092-915-4, 2006, 2006a, p. 165

I. Negueruela, D.M. Smith, T.E. Harrison, J.M. Torrejón, The Optical Counterpart to the Peculiar X-Ray Transient XTE J1739-302. ApJ 638, 982-986 (2006b). doi:10.1086/498935

I. Negueruela, D.M. Smith, J.M. Torrejón, P. Reig, Supergiant Fast X-Ray Transients: A Common Behaviour or a Class of Objects?, in ESA Special Publication. ESA Special Publication, vol. 622, 2007, p. 255

I. Negueruela, J. Casares, F. Verrecchia, P. Blay, G.L. Israel, S. Covino, XTE J1855-026 is a supergiant X-ray binary. The Astronomer's Telegram 1876, 1 (2008)

I. Negueruela, J. Lorenzo, A. Herrero, A.J. Norton, HD 306414: Optical Counterpart to the Peculiar X-ray Transient IGR J11215-5952, in Binaries - Key to Comprehension of the Universe, ed. by A. Prša, M. Zejda Astronomical Society of the Pacific Conference Series, vol. 435, 2010, p. 407

E. Nespoli, J. Fabregat, R.E. Mennickent, Unveiling the nature of six HMXBs through IR spectroscopy. A\&A 486, 911-917 (2008). doi:10.1051/0004-6361:200809645

E. Nespoli, J. Fabregat, R.E. Mennickent, Unveiling the nature of IGR J16493-4348 with IR spectroscopy. A\&A 516, 106 (2010). doi:10.1051/0004-6361/201014348

M. Newman, Power laws, Pareto distributions and Zipf's law. Contemporary Physics 46, 
323-351 (2005). doi:10.1080/00107510500052444

U.M. Noebauer, S.A. Sim, Self-consistent modelling of line-driven hot-star winds with Monte Carlo radiation hydrodynamics. MNRAS 453, 3120-3134 (2015). doi:10.1093/mnras/stv1849

T. Nugis, H.J.G.L.M. Lamers, The mass-loss rates of wolf-rayet stars explained by optically thick radiation driven wind models. A\&A 389, 162-179 (2002)

G.I. Ogilvie, G. Dubus, Precessing warped accretion discs in X-ray binaries. MNRAS 320, 485-503 (2001). doi:10.1046/j.1365-8711.2001.04011.x

L.M. Oskinova, A. Feldmeier, W.-R. Hamann, X-ray emission lines from inhomogeneous stellar winds. A\&A 422, 675-691 (2004). doi:10.1051/0004-6361:20047187

L.M. Oskinova, A. Feldmeier, W. Hamann, High-resolution X-ray spectroscopy of bright O-type stars. MNRAS 372, 313-326 (2006). doi:10.1111/j.1365-2966.2006.10858.x

L.M. Oskinova, A. Feldmeier, P. Kretschmar, Clumped stellar winds in supergiant highmass X-ray binaries: X-ray variability and photoionization. MNRAS 421, 2820-2831 (2012). doi:10.1111/j.1365-2966.2012.20507.x

L.M. Oskinova, W. Hamann, A. Feldmeier, Neglecting the porosity of hot-star winds can lead to underestimating mass-loss rates. A\&A 476, 1331-1340 (2007). doi:10.1051/00046361:20066377

L. Oskinova, W.-R. Hamann, R. Ignace, A. Feldmeier, X-rays, clumping and wind structures. Bulletin de la Societe Royale des Sciences de Liege 80, 54-66 (2011)

S.P. Owocki, Co-Rotating Interaction Regions in 2D Hot-Star Wind Models with LineDriven Instability, in IAU Colloq. 169: Variable and Non-spherical Stellar Winds in Luminous Hot Stars, ed. by B. Wolf, O. Stahl, A.W. Fullerton Lecture Notes in Physics, Berlin Springer Verlag, vol. 523, 1999, p. 294

S.P. Owocki, Dynamical simulation of the "velocity-porosity" reduction in observed strength of stellar wind lines, in Clumping in Hot-Star Winds, ed. by W.-R. Hamann, A. Feldmeier, L.M. Oskinova, 2008, p. 121

S.P. Owocki, D.H. Cohen, X-Ray Line Profiles from Parameterized Emission within an Accelerating Stellar Wind. ApJ 559, 1108-1116 (2001). doi:10.1086/322413

S.P. Owocki, D.H. Cohen, The Effect of Porosity on X-Ray Emission-Line Profiles from Hot-Star Winds. ApJ 648, 565-571 (2006). doi:10.1086/505698

S.P. Owocki, J. Puls, Nonlocal Escape-Integral Approximations for the Line Force in Structured Line-driven Stellar Winds. ApJ 462, 894 (1996). doi:10.1086/177203

S.P. Owocki, J. Puls, Line-driven Stellar Winds: The Dynamical Role of Diffuse Radiation Gradients and Limitations to the Sobolev Approach. ApJ 510, 355-368 (1999)

S.P. Owocki, J. Puls, Ion Runaway Instability in Low-Density, Line-driven Stellar Winds. ApJ 568, 965-978 (2002)

S.P. Owocki, G.B. Rybicki, Instabilities in line-driven stellar winds. I - Dependence on perturbation wavelength. ApJ 284, 337-350 (1984). doi:10.1086/162412

S.P. Owocki, G.B. Rybicki, Instabilities in line-driven stellar winds. II - Effect of scattering. ApJ 299, 265-276 (1985). doi:10.1086/163697

S.P. Owocki, J.I. Castor, G.B. Rybicki, Time-dependent models of radiatively driven stellar winds. I - Nonlinear evolution of instabilities for a pure absorption model. ApJ 335, 914930 (1988). doi:10.1086/166977

S.P. Owocki, S.R. Cranmer, A.W. Fullerton, Periodic Variations in Ultraviolet Spectral Lines of the B0.5 Ib Star HD 64760: Evidence for Corotating Wind Streams Rooted in Surface Variations. ApJ 453, 37 (1995)

S.P. Owocki, K.G. Gayley, N.J. Shaviv, A Porosity-Length Formalism for Photon-Tiringlimited Mass Loss from Stars above the Eddington Limit. ApJ 616, 525-541 (2004) 
S.P. Owocki, J.O. Sundqvist, D.H. Cohen, K.G. Gayley, Thin-shell mixing in radiative wind-shocks and the Lx-Lbol scaling of O-star X-rays. MNRAS 429, 3379-3389 (2013). doi: $10.1093 / \mathrm{mnras} / \mathrm{sts} 599$

A. Paizis, L. Sidoli, Cumulative luminosity distributions of supergiant fast X-ray transients in hard X-rays. MNRAS 439, 3439-3452 (2014). doi:10.1093/mnras/stu191

P. Palmeri, C. Mendoza, T.R. Kallman, M.A. Bautista, M. Meléndez, Modeling of iron K lines: Radiative and Auger decay data for Fe II-Fe IX. A\&A 410, 359-364 (2003). doi:10.1051/0004-6361:20031262

A. Pauldrach, Radiation driven winds of hot luminous stars. III - Detailed statistical equilibrium calculations for hydrogen to zinc. A\&A 183, 295-313 (1987)

A. Pauldrach, J. Puls, R.P. Kudritzki, Radiation-driven winds of hot luminous stars - Improvements of the theory and first results. A\&A 164, 86-100 (1986)

A.W.A. Pauldrach, J. Puls, Radiation-driven winds of hot stars. VIII - The bistable wind of the luminous blue variable P Cygni (B1 Ia/+/). A\&A 237, 409-424 (1990)

A.W.A. Pauldrach, T.L. Hoffmann, M. Lennon, Radiation-driven winds of hot luminous stars. XIII. A description of NLTE line blocking and blanketing towards realistic models for expanding atmospheres. A\&A 375, 161-195 (2001). doi:10.1051/00046361:20010805

A.W.A. Pauldrach, T.L. Hoffmann, R.H. Méndez, Radiation Driven Atmospheres of O-type stars: Constraints on the Mass-Luminosity Relation of Central Stars of Planetary Nebulae (invited review), in Planetary Nebulae: Their Evolution and Role in the Universe, ed. by S. Kwok, M. Dopita, R. Sutherland IAU Symposium, vol. 209, 2003, p. 177

A.W.A. Pauldrach, R.P. Kudritzki, J. Puls, K. Butler, J. Hunsinger, Radiation-driven winds of hot luminous stars. 12: A first step towards detailed UV-line diagnostics of O-stars. A\&A 283, 525-560 (1994)

A.B. Pearlman, R. Corbet, K. Pottschmidt, Superorbital Modulation and Orbital Parameters of the Eclipsing High-Mass X-ray Pulsar IGR J16493-4348, in American Astronomical Society Meeting Abstracts 221. American Astronomical Society Meeting Abstracts, vol. 221, 2013, pp. 142-38

A.B. Pearlman, R.H.D. Corbet, K. Pottschmidt, G.K. Skinner, The Orbital Parameters and Nature of the X-ray Pulsar IGR J16393-4643 Using Pulse Timing Analysis, in AAS/High Energy Astrophysics Division. AAS/High Energy Astrophysics Division, vol. 12, 2011, pp. 42-06

V. Petit, S.P. Owocki, G.A. Wade, D.H. Cohen, J.O. Sundqvist, M. Gagné, J. Maíz Apellániz, M.E. Oksala, D.A. Bohlender, T. Rivinius, H.F. Henrichs, E. Alecian, R.H.D. Townsend, A. ud-Doula, MiMeS Collaboration, A magnetic confinement versus rotation classification of massive-star magnetospheres. MNRAS 429, 398-422 (2013). doi:10.1093/mnras/sts344

P. Petrenz, J. Puls, H $\alpha$ line formation in hot star winds: the influence of rotation. A\&A 312, 195-220 (1996)

B. Petrov, J.S. Vink, G. Gräfener, On the $\mathrm{H} \alpha$ behaviour of blue supergiants: rise and fall over the bi-stability jump. A\&A 565, 62 (2014). doi:10.1051/0004-6361/201322754

B.K. Prinja, UV P Cygni Profile Variability in 0 Stars (Invited Paper), in Nonisotropic and Variable Outflows from Stars, ed. by L. Drissen, C. Leitherer, A. Nota Astronomical Society of the Pacific Conference Series, vol. 22, 1992, p. 167

R.K. Prinja, Evidence for rotationally modulated variability in O star winds. MNRAS 231, 21-24 (1988)

R.K. Prinja, Time-Dependent Phenomena in OB Star Winds, in Pulsation; Rotation; and Mass Loss in Early-Type Stars, ed. by L.A. Balona, H.F. Henrichs, J.M. Le Contel IAU 
Symposium, vol. 162, 1994, p. 507

R.K. Prinja, D.L. Massa, Signature of wide-spread clumping in B supergiant winds. A\&A 521, 55 (2010). doi:10.1051/0004-6361/201015252

R.K. Prinja, D.L. Massa, Ultraviolet diagnostic of porosity-free mass-loss estimates in B stars. A\&A 559, 15 (2013). doi:10.1051/0004-6361/201321799

R.K. Prinja, L.J. Smith, Migrating optical depth enhancements in the UV wind lines of the Wolf-Rayet star HD 93131. A\&A 266, 377-384 (1992)

R.K. Prinja, M.J. Barlow, I.D. Howarth, Terminal velocities for a large sample of O stars, B supergiants, and Wolf-Rayet stars. ApJ 361, 607-620 (1990)

R.K. Prinja, D. Massa, A.W. Fullerton, The IUE MEGA Campaign: Modulated Structure in the Wind of HD 64760 (B0.5 Ib). ApJ 452, 61 (1995)

R.K. Prinja, L.A. Balona, C.T. Bolton, R.A. Crowe, M.S. Fieldus, A.W. Fullerton, D.R. Gies, I.D. Howarth, D. McDavid, A.H.N. Reid, Time series observations of O stars. I - IUE observations of variability in the stellar wind of Zeta Puppis. ApJ 390, 266-272 (1992)

N. Przybilla, M. Nieva, K. Butler, A Cosmic Abundance Standard: Chemical Homogeneity of the Solar Neighborhood and the ISM Dust-Phase Composition. ApJ 688, 103-106 (2008). doi:10.1086/595618

J. Puls, Radiation-driven winds of hot luminous stars. IV - The influence of multi-line effects. A\&A 184, 227-248 (1987)

J. Puls, U. Springmann, M. Lennon, Radiation driven winds of hot luminous stars. XIV. Line statistics and radiative driving. A\&AS 141, 23-64 (2000)

J. Puls, J.O. Sundqvist, J.G. Rivero González, OB-stars as extreme condition test beds. ArXiv e-prints (2010)

J. Puls, J.S. Vink, F. Najarro, Mass loss from hot massive stars. A\&A Rev. 16, 209-325 (2008)

J. Puls, R.-P. Kudritzki, A. Herrero, A.W.A. Pauldrach, S.M. Haser, D.J. Lennon, R. Gabler, S.A. Voels, J.M. Vilchez, S. Wachter, A. Feldmeier, O-star mass-loss and wind momentum rates in the Galaxy and the Magellanic Clouds Observations and theoretical predictions. A\&A 305, 171 (1996)

J. Puls, M.A. Urbaneja, R. Venero, T. Repolust, U. Springmann, A. Jokuthy, M.R. Mokiem, Atmospheric NLTE-models for the spectroscopic analysis of blue stars with winds. II. Line-blanketed models. A\&A 435, 669-698 (2005)

J. Puls, N. Markova, S. Scuderi, C. Stanghellini, O.G. Taranova, A.W. Burnley, I.D. Howarth, Bright OB stars in the Galaxy. III. Constraints on the radial stratification of the clumping factor in hot star winds from a combined $\mathrm{H}$, IR and radio analysis. A\&A 454, 625-651 (2006). doi:10.1051/0004-6361:20065073

H. Quaintrell, A.J. Norton, T.D.C. Ash, P. Roche, B. Willems, T.R. Bedding, I.K. Baldry, R.P. Fender, The mass of the neutron star in Vela X-1 and tidally induced non-radial oscillations in GP Vel. A\&A 401, 313-323 (2003). doi:10.1051/0004-6361:20030120

F. Rahoui, S. Chaty, IGR J18483-0311: a new intermediate supergiant fast X-ray transient. A\&A 492, 163-166 (2008). doi:10.1051/0004-6361:200810695

F. Rahoui, S. Chaty, P.-O. Lagage, E. Pantin, Multi-wavelength observations of Galactic hard X-ray sources discovered by INTEGRAL. II. The environment of the companion star. A\&A 484, 801-813 (2008). doi:10.1051/0004-6361:20078774

R.A. Rampy, D.M. Smith, I. Negueruela, IGR J17544-2619 in Depth With Suzaku: Direct Evidence for Clumpy Winds in a Supergiant Fast X-ray Transient. ApJ 707, 243-249 (2009). doi:10.1088/0004-637X/707/1/243

G. Rauw, A. Herve, Y. Naze, J.N. Gonzalez-Perez, A. Hempelmann, M. Mittag, J.H.M.M. 
Schmitt, K.-P. Schroeder, E. Gosset, P. Eenens, J.M. Uuh-Sonda, Simultaneous X-ray and optical spectroscopy of the Oef supergiant lambda Cep. ArXiv e-prints (2015)

P.S. Ray, D. Chakrabarty, The Orbit of the High-Mass X-Ray Binary Pulsar 1E 1145.1-6141. ApJ 581, 1293-1296 (2002). doi:10.1086/344300

N. Rea, P. Esposito, Magnetar outbursts: an observational review, in High-Energy Emission from Pulsars and their Systems, ed. by D.F. Torres, N. Rea, 2011, p. 247. doi:10.1007/9783-642-17251-9_21

P. Reig, D. Chakrabarty, M.J. Coe, J. Fabregat, I. Negueruela, T.A. Prince, P. Roche, I.A. Steele, Astrophysical parameters of the massive X-ray binary 2S 0114+650. A\&A 311, 879-888 (1996)

T. Repolust, J. Puls, A. Herrero, Stellar and wind parameters of Galactic O-stars. The influence of line-blocking/blanketing. A\&A 415, 349-376 (2004)

A.P. Reynolds, S.A. Bell, R.W. Hilditch, Optical spectroscopy of the massive X-ray binary QV Nor (4U 1538 - 52). MNRAS 256, 631-640 (1992)

J.G. Rivero González, J. Puls, F. Najarro, I. Brott, Nitrogen line spectroscopy of O-stars. II. Surface nitrogen abundances for O-stars in the Large Magellanic Cloud. A\&A 537, 79 (2012). doi:10.1051/0004-6361/201117790

E. Rivers, A. Markowitz, K. Pottschmidt, S. Roth, L. Barragán, F. Fürst, S. Suchy, I. Kreykenbohm, J. Wilms, R. Rothschild, A Comprehensive Spectral Analysis of the X-Ray Pulsar 4U 1907+09 from Two Observations with the Suzaku X-ray Observatory. ApJ 709, 179-190 (2010). doi:10.1088/0004-637X/709/1/179. http://cdsads.ustrasbg.fr/abs/2010ApJ...709..179R

M.S.E. Roberts, P.F. Michelson, D.A. Leahy, T.A. Hall, J.P. Finley, L.R. Cominsky, R. Srinivasan, Phase-dependent Spectral Variability in 4U 1907+09. ApJ 555, 967-977 (2001). doi:10.1086/321487

J. Rodriguez, A. Bodaghee, P. Kaaret, J.A. Tomsick, E. Kuulkers, G. Malaguti, P.-O. Petrucci, C. Cabanac, M. Chernyakova, S. Corbel, S. Deluit, G. Di Cocco, K. Ebisawa, A. Goldwurm, G. Henri, F. Lebrun, A. Paizis, R. Walter, L. Foschini, INTEGRAL and XMM-Newton observations of the X-ray pulsar IGR J16320-4751/AX J1631.9-4752. MNRAS 366, 274-282 (2006)

P. Romano, L. Sidoli, G. Cusumano, S. Vercellone, V. Mangano, H.A. Krimm, Disentangling the System Geometry of the Supergiant Fast X-Ray Transient IGR J11215-5952 with Swift. ApJ 696, 2068-2074 (2009a). doi:10.1088/0004-637X/696/2/2068

P. Romano, L. Sidoli, G. Cusumano, V. La Parola, S. Vercellone, C. Pagani, L. Ducci, V. Mangano, J. Cummings, H.A. Krimm, C. Guidorzi, J.A. Kennea, E.A. Hoversten, D.N. Burrows, N. Gehrels, Monitoring supergiant fast X-ray transients with Swift: results from the first year. MNRAS 399, 2021-2032 (2009b). doi:10.1111/j.1365-2966.2009.15356.x

P. Romano, L. Sidoli, L. Ducci, G. Cusumano, V. La Parola, C. Pagani, K.L. Page, J.A. Kennea, D.N. Burrows, N. Gehrels, V. Sguera, A. Bazzano, Swift/XRT monitoring of the supergiant fast X-ray transient IGR J18483-0311 for an entire orbital period. MNRAS 401, 1564-1569 (2010). doi:10.1111/j.1365-2966.2009.15789.x

P. Romano, V. La Parola, S. Vercellone, G. Cusumano, L. Sidoli, H.A. Krimm, C. Pagani, P. Esposito, E.A. Hoversten, J.A. Kennea, K.L. Page, D.N. Burrows, N. Gehrels, Two years of monitoring supergiant fast X-ray transients with Swift. MNRAS 410, 1825-1836 (2011). doi:10.1111/j.1365-2966.2010.17564.X

P. Romano, V. Mangano, L. Ducci, P. Esposito, P.A. Evans, S. Vercellone, J.A. Kennea, D.N. Burrows, N. Gehrels, Swift/X-ray Telescope monitoring of the candidate supergiant fast X-ray transient IGR J16418-4532. MNRAS 419, 2695-2702 (2012). doi:10.1111/j.13652966.2011.19916.x 
P. Romano, L. Ducci, V. Mangano, P. Esposito, E. Bozzo, S. Vercellone, Soft X-ray characterisation of the long-term properties of supergiant fast X-ray transients. A\&A 568, 55 (2014a). doi:10.1051/0004-6361/201423867

P. Romano, H.A. Krimm, D.M. Palmer, L. Ducci, P. Esposito, S. Vercellone, P.A. Evans, C. Guidorzi, V. Mangano, J.A. Kennea, S.D. Barthelmy, D.N. Burrows, N. Gehrels, The 100month Swift catalogue of supergiant fast X-ray transients. I. BAT on-board and transient monitor flares. A\&A 562, 2 (2014b). doi:10.1051/0004-6361/201322516

P. Romano, E. Bozzo, V. Mangano, P. Esposito, G. Israel, A. Tiengo, S. Campana, L. Ducci, C. Ferrigno, J.A. Kennea, Giant outburst from the supergiant fast X-ray transient IGR J17544-2619: accretion from a transient disc? A\&A 576, 4 (2015). doi:10.1051/0004$6361 / 201525749$

M.M. Romanova, O.D. Toropina, Y.M. Toropin, R.V.E. Lovelace, Magnetohydrodynamic Simulations of Accretion onto a Star in the "Propeller" Regime. ApJ 588, 400-407 (2003). doi:10.1086/373990

M.M. Romanova, G.V. Ustyugova, A.V. Koldoba, R.V.E. Lovelace, MRI-driven accretion on to magnetized stars: global 3D MHD simulations of magnetospheric and boundary layer regimes. MNRAS 421, 63-77 (2012). doi:10.1111/j.1365-2966.2011.20055.x

M.C. Runacres, S.P. Owocki, The outer evolution of instability-generated structure in radiatively driven stellar winds. A\&A 381, 1015-1025 (2002). doi:10.1051/00046361:20011526

G.B. Rybicki, S.P. Owocki, J.I. Castor, Instabilities in line-driven stellar winds. IV - Linear perturbations in three dimensions. ApJ 349, 274-285 (1990). doi:10.1086/168312

M. Sako, S.M. Kahn, F. Paerels, D.A. Liedahl, S. Watanabe, F. Nagase, T. Takahashi, Structure and Dynamics of Stellar Winds in High-mass X-ray Binaries. ArXiv 0309503 (2003)

A. Sander, W.-R. Hamann, H. Todt, The Galactic WC stars. Stellar parameters from spectral analyses indicate a new evolutionary sequence. A\&A 540, 144 (2012). doi:10.1051/0004$6361 / 201117830$

G. Schönherr, F.-W. Schwarm, S. Falkner, T. Dauser, C. Ferrigno, M. Kühnel, D. Klochkov, P. Kretschmar, P.A. Becker, M.T. Wolff, K. Pottschmidt, M. Falanga, I. Kreykenbohm, F. Fürst, R. Staubert, J. Wilms, Formation of phase lags at the cyclotron energies in the pulse profiles of magnetized, accreting neutron stars. A\&A 564, 8 (2014)

V. Sguera, E.J. Barlow, A.J. Bird, D.J. Clark, A.J. Dean, A.B. Hill, L. Moran, S.E. Shaw, D.R. Willis, A. Bazzano, P. Ubertini, A. Malizia, INTEGRAL observations of recurrent fast X-ray transient sources. A\&A 444, 221-231 (2005). doi:10.1051/00046361:20053103

V. Sguera, A. Bazzano, A.J. Bird, A.J. Dean, P. Ubertini, E.J. Barlow, L. Bassani, D.J. Clark, A.B. Hill, A. Malizia, M. Molina, J.B. Stephen, Unveiling Supergiant Fast X-Ray Transient Sources with INTEGRAL. ApJ 646, 452-463 (2006). doi:10.1086/504827

V. Sguera, A.B. Hill, A.J. Bird, A.J. Dean, A. Bazzano, P. Ubertini, N. Masetti, R. Landi, A. Malizia, D.J. Clark, M. Molina, IGR J18483-0311: an accreting X-ray pulsar observed by INTEGRAL. A\&A 467, 249-257 (2007). doi:10.1051/0004-6361:20066762

V. Sguera, L. Ducci, L. Sidoli, A. Bazzano, L. Bassani, XMM-Newton and INTEGRAL study of the SFXT IGR J18483-0311 in quiescence: hint of a cyclotron emission feature? MNRAS 402, 49-53 (2010). doi:10.1111/j.1745-3933.2009.00798.x

V. Sguera, L. Sidoli, A.J. Bird, A. Bazzano, INTEGRAL discovery of unusually long broadband X-ray activity from the Supergiant Fast X-ray Transient IGR J18483-0311. MNRAS 449, 1228-1237 (2015). doi:10.1093/mnras/stv341

N. Shakura, K. Postnov, L. Hjalmarsdotter, On the nature of 'off' states in slowly rotating low-luminosity X-ray pulsars. MNRAS 428, 670-677 (2013). doi:10.1093/mnras/sts062 
N. Shakura, K. Postnov, A. Kochetkova, L. Hjalmarsdotter, Theory of quasi-spherical accretion in X-ray pulsars. MNRAS 420, 216-236 (2012). doi:10.1111/j.13652966.2011.20026.x

N. Shakura, k. Postnov, L. Sidoli, A. Paizis, Bright Flares in Supergiant Fast X-ray Transients. ArXiv e-prints, MNRAS in press (2014)

N.J. Shaviv, The Eddington Luminosity Limit for Multiphased Media. ApJ 494, 193-197 (1998). doi:10.1086/311182

N.J. Shaviv, The Porous Atmosphere of $\eta$ Carinae. ApJ 532, 137-140 (2000). doi: $10.1086 / 312585$

T. Shenar, L. Oskinova, W.-R. Hamann, M.F. Corcoran, A.F.J. Moffat, H. Pablo, N.D. Richardson, W.L. Waldron, D.P. Huenemoerder, J. Maíz Apellániz, J.S. Nichols, H. Todt, Y. Nazé, J.L. Hoffman, A.M.T. Pollock, I. Negueruela, A coordinated X-ray and Optical Campaign of the Nearest Massive Eclipsing Binary, $\$ \backslash$ delta $\$$ Orionis Aa: IV. A multiwavelength, non-LTE spectroscopic analysis. ArXiv e-prints (2015)

J.M. Shull, S. Beckwith, Interstellar molecular hydrogen. ARA\&A 20, 163-190 (1982)

L. Sidoli, Supergiant Fast X-ray Transients: a review, in Proceedings of "An INTEGRAL view of the high-energy sky (the first 10 years)" - 9th INTEGRAL Workshop and celebration of the 10th anniversary of the launch (INTEGRAL 2012). 15-19 October 2012. Bibliotheque Nationale de France, Paris, France. Published online at iA href="http://pos.sissa.it/cgi-bin/reader/conf.cgi?confid=176” ¿http://pos.sissa.it/cgibin/reader/conf.cgi? confid $=176_{j} / A_{i}$, id.11, 2012, p. 11

L. Sidoli, P. Esposito, L. Ducci, The longest observation of a low-intensity state from a supergiant fast X-ray transient: Suzaku observes IGRJ08408-4503. MNRAS 409, 611618 (2010). doi:10.1111/j.1365-2966.2010.17320.x

L. Sidoli, A. Paizis, S. Mereghetti, IGR J11215-5952: a hard X-ray transient displaying recurrent outbursts. A\&A 450, 9-12 (2006). doi:10.1051/0004-6361:20064940

L. Sidoli, P. Romano, S. Mereghetti, A. Paizis, S. Vercellone, V. Mangano, D. Götz, An alternative hypothesis for the outburst mechanism in supergiant fast X-ray transients: the case of IGR J11215-5952. A\&A 476, 1307-1315 (2007). doi:10.1051/0004-6361:20078137

L. Sidoli, P. Romano, V. Mangano, A. Pellizzoni, J.A. Kennea, G. Cusumano, S. Vercellone, A. Paizis, D.N. Burrows, N. Gehrels, Monitoring Supergiant Fast X-ray Transients with Swift. I. Behavior outside outbursts. ApJ 687, 1230-1235 (2008)

L. Sidoli, S. Mereghetti, V. Sguera, F. Pizzolato, The XMM-Newton view of supergiant fast X-ray transients: the case of IGR J16418-4532. MNRAS 420, 554-561 (2012). doi:10.1111/j.1365-2966.2011.20063.X

L. Sidoli, P. Esposito, V. Sguera, A. Bodaghee, J.A. Tomsick, K. Pottschmidt, J. Rodriguez, P. Romano, J. Wilms, A Suzaku X-ray observation of one orbit of the supergiant fast X-ray transient IGR J16479-4514. MNRAS 429, 2763-2771 (2013). doi:10.1093/mnras/sts559

L. Sidoli, A. Paizis, F. Fürst, J.M. Torrejón, P. Kretschmar, E. Bozzo, K. Pottschmidt, Probing large-scale wind structures in Vela X-1 using off-states with INTEGRAL. MNRAS 447, 1299-1303 (2015). doi:10.1093/mnras/stu2533

S. Simón-Díaz, G. Stasińska, The ionizing radiation from massive stars and its impact on HII regions: results from modern model atmospheres. MNRAS 389, 1009-1021 (2008). doi:10.1111/j.1365-2966.2008.13444.x

S. Simón-Díaz, N. Castro, A. Herrero, J. Puls, M. Garcia, C. Sabín-Sanjulián, The IACOB project: A grid-based automatic tool for the quantitative spectroscopic analysis of Ostars. Journal of Physics Conference Series 328(1), 012021 (2011). doi:10.1088/1742$6596 / 328 / 1 / 012021$

D.M. Smith, C.B. Markwardt, J.H. Swank, I. Negueruela, Fast X-ray transients towards the 
Galactic bulge with the Rossi X-ray Timing Explorer. MNRAS 422, 2661-2674 (2012). doi:10.1111/j.1365-2966.2012.20836.x

N. Smith, S.P. Owocki, On the Role of Continuum-driven Eruptions in the Evolution of Very Massive Stars and Population III Stars. ApJ 645, 45-48 (2006). doi:10.1086/506523

V.V. Sobolev, Moving envelopes of stars (Cambridge: Harvard University Press, 1960, ???, 1960)

U.W.E. Springmann, A.W.A. Pauldrach, Radiation-driven winds of hot luminous stars. XI Frictional heating in a multicomponent stellar wind plasma and decoupling of radiatively accelerated ions. A\&A 262, 515-522 (1992)

I.R. Stevens, X-ray-illuminated stellar winds - Optically thick wind models for massive Xray binaries. ApJ 379, 310-326 (1991). doi:10.1086/170506

G.C. Stewart, A.C. Fabian, The influence of mass loss on the observed X-ray spectra of early-type stars. MNRAS 197, 713-720 (1981)

J.O. Sundqvist, S.P. Owocki, Clumping in the inner winds of hot, massive stars from hydrodynamical line-driven instability simulations. MNRAS 428, 1837-1844 (2013). doi:10.1093/mnras/sts 165

J.O. Sundqvist, S.P. Owocki, J. Puls, The nature and consequences of clumping in hot, massive star winds. ArXiv 1110.0485 (2011)

J.O. Sundqvist, J. Puls, A. Feldmeier, Mass loss from inhomogeneous hot star winds. I. Resonance line formation in 2D models. A\&A 510, 11 (2010). doi:10.1051/00046361/200912842

J.O. Sundqvist, J. Puls, S.P. Owocki, Mass loss from inhomogeneous hot star winds. III. An effective-opacity formalism for line radiative transfer in accelerating, clumped twocomponent media, and first results on theory and diagnostics. A\&A 568, 59 (2014). doi:10.1051/0004-6361/201423570

J.O. Sundqvist, J. Puls, A. Feldmeier, S.P. Owocki, Mass loss from inhomogeneous hot star winds. II. Constraints from a combined optical/UV study. A\&A 528, 64 (2011). doi:10.1051/0004-6361/201015771

J.O. Sundqvist, A. ud-Doula, S.P. Owocki, R.H.D. Townsend, I.D. Howarth, G.A. Wade, A dynamical magnetosphere model for periodic $\mathrm{H} \alpha$ emission from the slowly rotating magnetic O star HD 191612. MNRAS 423, 21-25 (2012a). doi:10.1111/j.17453933.2012.01248.x

J.O. Sundqvist, S.P. Owocki, D.H. Cohen, M.A. Leutenegger, R.H.D. Townsend, A generalized porosity formalism for isotropic and anisotropic effective opacity and its effects on X-ray line attenuation in clumped O star winds. MNRAS 420, 1553-1561 (2012b). doi:10.1111/j.1365-2966.2011.20141.x

J.O. Sundqvist, S. Simón-Díaz, J. Puls, N. Markova, The rotation rates of massive stars. How slow are the slow ones? A\&A 559, 10 (2013). doi:10.1051/0004-6361/201322761

R.A. Sunyaev, S.A. Grebenev, A.A. Lutovinov, J. Rodriguez, S. Mereghetti, D. Gotz, T. Courvoisier, New source IGR J17544-2619 discovered with INTEGRAL. The Astronomer's Telegram 190, 1 (2003)

J.H. Swank, D.M. Smith, C.B. Markwardt, RXTE PCA Pointed Observations of IGR J11215-5952. The Astronomer's Telegram 999, 1 (2007)

C.B. Tarter, W.H. Tucker, E.E. Salpeter, The Interaction of X-Ray Sources with Optically Thin Environments. ApJ 156, 943 (1969). doi:10.1086/150026

H. Todt, M. Peña, W.-R. Hamann, G. Gräfener, The central star of the planetary nebula PB 8: a Wolf-Rayet-type wind of an unusual WN/WC chemical composition. A\&A 515, 83 (2010). doi:10.1051/0004-6361/200912183

Y.M. Toropin, O.D. Toropina, V.V. Savelyev, M.M. Romanova, V.M. Chechetkin, R.V.E. 
Lovelace, Spherical Bondi Accretion onto a Magnetic Dipole. ApJ 517, 906-918 (1999). doi: $10.1086 / 307229$

O.D. Toropina, M.M. Romanova, R.V.E. Lovelace, Spinning-down of moving magnetars in the propeller regime. MNRAS 371, 569-576 (2006). doi:10.1111/j.13652966.2006.10667.x

O.D. Toropina, M.M. Romanova, R.V.E. Lovelace, Bondi-Hoyle accretion on to a magnetized neutron star. MNRAS 420, 810-816 (2012). doi:10.1111/j.13652966.2011.20093.x

J.M. Torrejón, N.S. Schulz, M.A. Nowak, T.R. Kallman, A Chandra Survey of Fluorescence Fe Lines in X-ray Binaries at High Resolution. ApJ 715, 947-958 (2010a)

J.M. Torrejón, I. Negueruela, D.M. Smith, T.E. Harrison, Near-infrared survey of high mass X-ray binary candidates. A\&A 510, 61 (2010b). doi:10.1051/0004-6361/200912619

J.M. Torrejón, N.S. Schulz, M.A. Nowak, L. Oskinova, J.J. Rodes-Roca, T. Shenar, J. Wilms, On the Radial Onset of Clumping in the Wind of the B0I Massive Star QV Nor. ApJ 810, 102 (2015). doi:10.1088/0004-637X/810/2/102

A. ud-Doula, Stellar Winds, Magnetic Fields and Disks, in Lecture Notes in Physics, Berlin Springer Verlag, ed. by J.-P. Rozelot, C.. Neiner Lecture Notes in Physics, Berlin Springer Verlag, vol. 857, 2013, p. 207. doi:10.1007/978-3-642-30648-8_8

A. ud-Doula, S.P. Owocki, Dynamical Simulations of Magnetically Channeled Line-driven Stellar Winds. I. Isothermal, Nonrotating, Radially Driven Flow. ApJ 576, 413-428 (2002). doi:10.1086/341543

J. Čechura, P. Hadrava, Stellar wind in state transitions of high-mass X-ray binaries. A\&A 575, 5 (2015). doi:10.1051/0004-6361/201424636

B. Šurlan, W.-R. Hamann, J. Kubát, L.M. Oskinova, A. Feldmeier, Three-dimensional radiative transfer in clumped hot star winds. I. Influence of clumping on the resonance line formation. A\&A 541, 37 (2012). doi:10.1051/0004-6361/201118590

B. Šurlan, W.-R. Hamann, A. Aret, J. Kubát, L.M. Oskinova, A.F. Torres, Macroclumping as solution of the discrepancy between $\mathrm{H} \alpha$ and $\mathrm{P} v$ mass loss diagnostics for O-type stars. A\&A 559, 130 (2013). doi:10.1051/0004-6361/201322390

A. van der Meer, L. Kaper, T. di Salvo, M. Méndez, M. van der Klis, P. Barr, N.R. Trams, XMM-Newton X-ray spectroscopy of the high-mass X-ray binary 4U 1700-37 at low flux. A\&A 432, 999-1012 (2005). doi:10.1051/0004-6361:20041288

J.T. van Loon, L. Kaper, G. Hammerschlag-Hensberge, Modelling the orbital modulation of ultraviolet resonance lines in high-mass X-ray binaries. A\&A 375, 498-526 (2001). doi:10.1051/0004-6361:20010856

D.A. Verner, G.J. Ferland, K.T. Korista, D.G. Yakovlev, Atomic data for astrophysics. ii. new analytic fits for photoionization cross sections of atoms and ions. ApJ 465, 487 (1996)

J.S. Vink, A. de Koter, H.J.G.L.M. Lamers, New theoretical mass-loss rates of O and B stars. A\&A 362, 295-309 (2000)

J.S. Vink, A. de Koter, H.J.G.L.M. Lamers, Mass-loss predictions for O and B stars as a function of metallicity. A\&A 369, 574-588 (2001)

J.S. Vink, I. Brott, G. Gräfener, N. Langer, A. de Koter, D.J. Lennon, The nature of B supergiants: clues from a steep drop in rotation rates at $22000 \mathrm{~K}$. The possibility of Bistability braking. A\&A 512, 7 (2010). doi:10.1051/0004-6361/201014205

J.S. Vink, L.E. Muijres, B. Anthonisse, A. de Koter, G. Gräfener, N. Langer, Wind modelling of very massive stars up to 300 solar masses. A\&A 531, 132 (2011). doi:10.1051/0004$6361 / 201116614$

G.A. Wade, J.H. Grunhut, MiMeS Collaboration, The MiMeS Survey of Magnetism in Mas- 
sive Stars, in Circumstellar Dynamics at High Resolution, ed. by A.C. Carciofi, T. Rivinius Astronomical Society of the Pacific Conference Series, vol. 464, 2012, p. 405

W.L. Waldron, J.P. Cassinelli, Chandra Discovers a Very High Density X-Ray Plasma on the O Star $\zeta$ Orionis. ApJ 548, 45-48 (2001). doi:10.1086/318926

W.L. Waldron, J.P. Cassinelli, An Extensive Collection of Stellar Wind X-Ray Source Region Emission Line Parameters, Temperatures, Velocities, and Their Radial Distributions as Obtained from Chandra Observations of 17 OB Stars. ApJ 668, 456-480 (2007). doi:10.1086/520919

R. Walter, J. Zurita Heras, Probing clumpy stellar winds with a neutron star. A\&A 476, 335-340 (2007)

R. Walter, J. Zurita Heras, L. Bassani, A. Bazzano, A. Bodaghee, A. Dean, P. Dubath, A.N. Parmar, M. Renaud, P. Ubertini, XMM-Newton and INTEGRAL observations of new absorbed supergiant high-mass X-ray binaries. A\&A 453, 133-143 (2006). doi:10.1051/0004-6361:20053719

R. Walter, A.A. Lutovinov, E. Bozzo, S.S. Tsygankov, High-Mass X-ray Binaries in the Milky Way: A closer look with INTEGRAL. ArXiv e-prints (2015)

W. Wang, Long-term hard X-ray monitoring of 2S 0114+65 with INTEGRAL/IBIS. MNRAS 413, 1083-1098 (2011). doi:10.1111/j.1365-2966.2010.18192.x

S. Watanabe, M. Sako, M. Ishida, Y. Ishisaki, S.M. Kahn, T. Kohmura, U. Morita, F. Nagase, F. Paerels, T. Takahashi, Detection of a Fully Resolved Compton Shoulder of the Iron $\mathrm{K} \alpha$ Line in the Chandra X-Ray Spectrum of GX 301-2. ApJ 597, 37-40 (2003). doi:10.1086/379735

S. Watanabe, M. Sako, M. Ishida, Y. Ishisaki, S.M. Kahn, T. Kohmura, F. Nagase, F. Paerels, T. Takahashi, X-Ray Spectral Study of the Photoionized Stellar Wind in Vela X-1. ApJ 651, 421-437 (2006). doi:10.1086/507458

N.E. White, S.H. Pravdo, The discovery of 38.22 second X-ray pulsations from the vicinity of OAO 1653-40. ApJ 233, 121-124 (1979). doi:10.1086/183089

N.E. White, R.H. Becker, S.H. Pravdo, E.A. Boldt, S.S. Holt, P.J. Serlemitsos, The X-ray pulsars 4U 1145-61 and 1E 1145.1-6141. ApJ 239, 655-660 (1980). doi:10.1086/158152

J. Wilms, A. Allen, R. McCray, On the absorption of X-rays in the interstellar medium. ApJ 542, 914-924 (2000)

A.E. Wright, M.J. Barlow, The radio and infrared spectrum of early-type stars undergoing mass loss. MNRAS 170, 41-51 (1975)

M. Yan, H.R. Sadeghpour, A. Dalgarno, Photoionization cross sections of he and $h_{2}$. ApJ 496, 1044-1050 (1998)

J.A. Zurita Heras, S. Chaty, Discovery of an eccentric 30 day period in the supergiant X-ray binary SAX J1818.6-1703 with INTEGRAL. A\&A 493, 1-4 (2009). doi:10.1051/00046361:200811179 